\title{
Experimental investigation of ion temperature anisotropy driven instabilities in a high beta plasma
}

\author{
Paul Arthur Keiter \\ West Virginia University
}

Follow this and additional works at: https://researchrepository.wvu.edu/etd

\section{Recommended Citation}

Keiter, Paul Arthur, "Experimental investigation of ion temperature anisotropy driven instabilities in a high beta plasma" (1999). Graduate Theses, Dissertations, and Problem Reports. 3180.

https://researchrepository.wvu.edu/etd/3180

This Dissertation is protected by copyright and/or related rights. It has been brought to you by the The Research Repository @ WVU with permission from the rights-holder(s). You are free to use this Dissertation in any way that is permitted by the copyright and related rights legislation that applies to your use. For other uses you must obtain permission from the rights-holder(s) directly, unless additional rights are indicated by a Creative Commons license in the record and/ or on the work itself. This Dissertation has been accepted for inclusion in WVU Graduate Theses, Dissertations, and Problem Reports collection by an authorized administrator of The Research Repository @ WVU.

For more information, please contact researchrepository@mail.wvu.edu. 


\title{
Experimental Investigation of Ion Temperature Anisotropy Driven Instabilities in a High Beta Plasma
}

\author{
Paul A. Keiter \\ Dissertation submitted to the College of Arts and Sciences \\ at West Virginia University \\ in partial fulfillment of the requirements \\ for the degree of \\ Doctor of Philosophy \\ in \\ Plasma Physics \\ Earl Scime, PhD., Chair \\ Mark Koepke, PhD. \\ Arthur Weldon, $\mathrm{PhD}$. \\ Richard Treat, $\mathrm{PhD}$, \\ Charter Stinespring, $\mathrm{PhD}$. \\ Department of Physics \\ Morgantown, West Virginia \\ 1999
}

Keywords: Ion Temperature Anisotropy, Helicon Source 


\section{AbStraCT \\ Experimental Investigation of Ion Temperature Anisotropy Driven Instabilities in a High Beta Plasma}

\section{Paul A. Keiter}

The first measurements of an ion temperature anisotropy/beta inverse correlation in a high beta laboratory experiment are presented. The observation of this correlation is similar to such a correlation present in in-situ spacecraft measurements and predicted in theory. Low-frequency fluctuations are also observed. According to measurements presented here, these waves are electromagnetic, transverse, occur at frequencies below the cyclotron frequency and have wavenumbers consistent with predictions for the ion cyclotron anisotropy instability, also referred to as the Alfvén Ion-Cyclotron instability. The device is a space simulation chamber that uses a helicon source for its plasma source. Ranges of ion beta and ion temperature anisotropy are $10^{-4}$ to $10^{-2}$ and 1 to 15 , respectively. The correlation is experimentally determined to be $T_{\perp} / T_{\|}=1+0.15 \beta_{i \|}^{-0.5}$. 


\section{ACKNOWLEDGEMENTS}

I would like to thank my advisor, Professor Earl Scime for his support and guidance throughout this project. I would also like to thank Professor Mark Koepke for helpful discussions and for development of the laser system. I would like to thank the other members of my committee, Professor Charter Stinespring, Professor Richard Treat and Professor Arthur Weldon for their contributions. Thanks to Mike Zintl for his guidance and assistance with the laser system. I would like to thank Doug Mathess, Tom Milan, and Carl Weber for the construction of the experiment and probes. Thanks also go to Matt Balkey and John Kline for their contributions to the development of the experiment and helpful discussions. I would also like to thank my family for their constant support throughout this work. 


\section{Table of Contents}

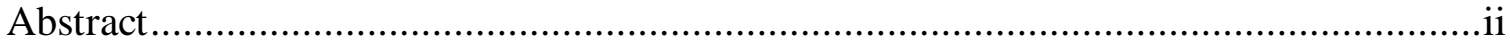

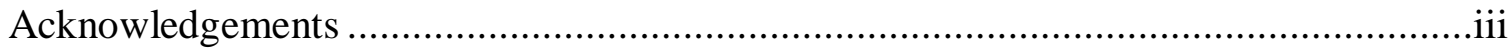

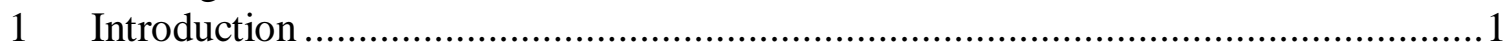

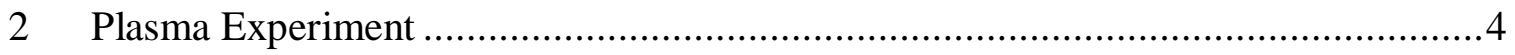

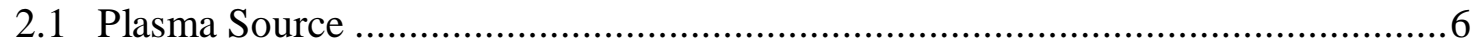

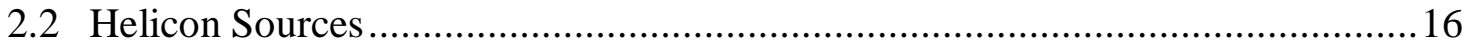

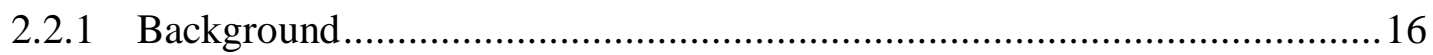

2.2.2 Helicon Dispersion Relation.......................................................... 22

2.2.3 Possible Explanations of Efficient Ionization in Helicon sources ................28

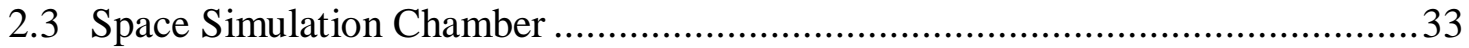

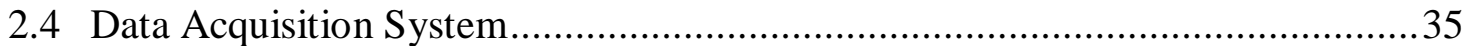

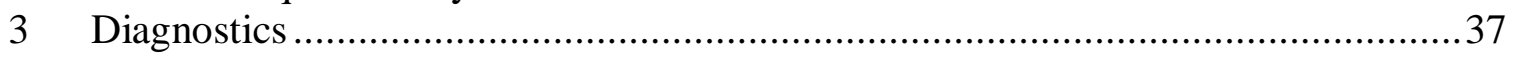

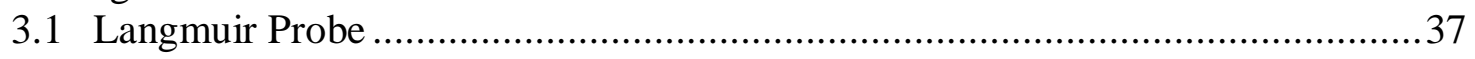

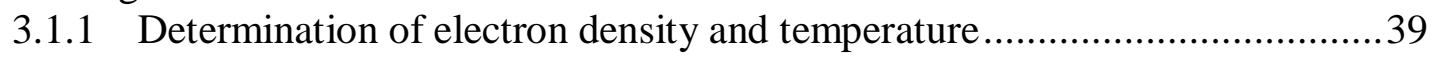

3.1.2 Langmuir Probe Design...................................................................... 40

3.2 Laser Induced Fluorescence .................................................................. 44

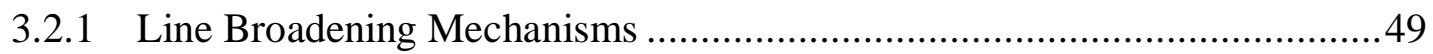

3.2.1.1 Natural Linewidth ...................................................................49

3.2.1.2 Doppler Broadening ...............................................................50

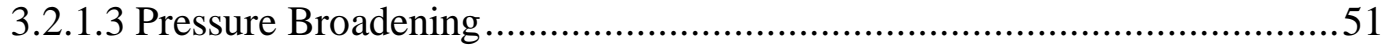

3.2.1.4 Zeeman Broadening ................................................................... 52

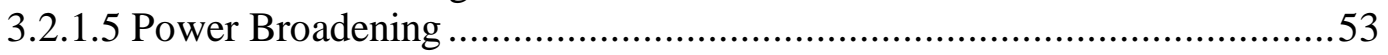

3.2.1.6 Instrumental Broadening .......................................................54

3.2.1.7 Comparison of Broadening Mechanisms......................................... 54

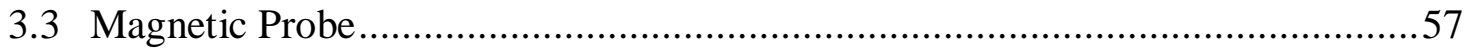

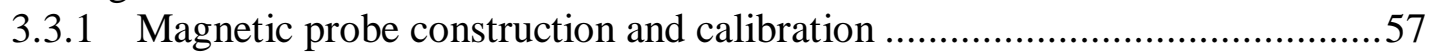

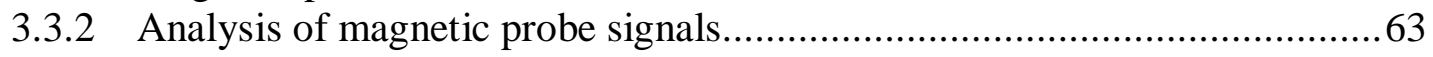

4 Electromagnetic Ion Temperature Anisotropy Driven Instabilities.......................68

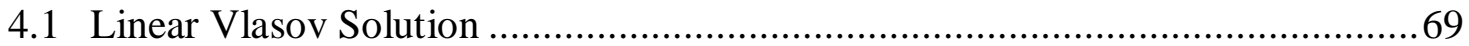

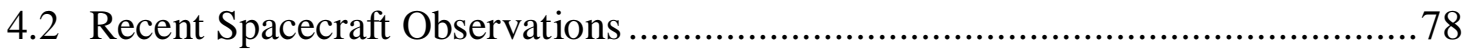

4.3 Previous Experimental Investigations of EMITA Instabilities .........................84

5 Experimental Results on Ion Temperature Anisotropy Upper Bound.....................86

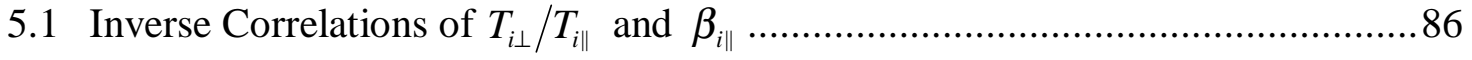

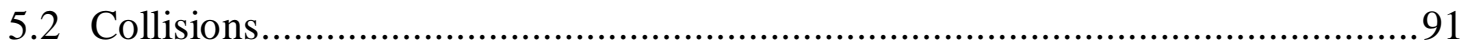

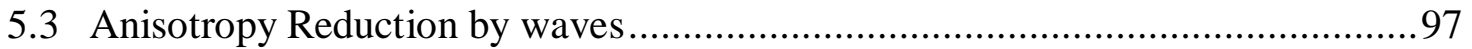

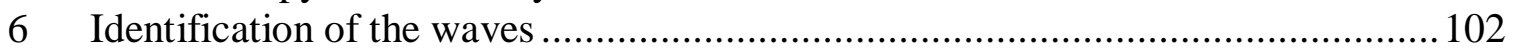

6.1 Wave Power Spectra and Amplitude Measurements...................................... 102

6.2 Wavenumber Measurements ....................................................................... 112

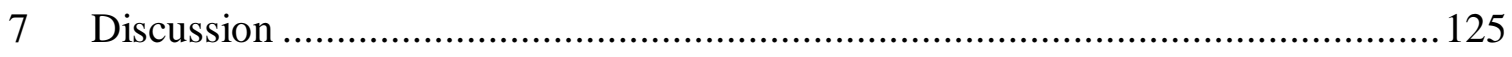

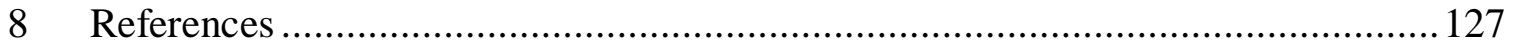




\section{INTRODUCTION}

The results presented in this thesis are from the first high-beta $\left(\beta=8 \pi n k T / B^{2}\right)$, steady-state laboratory experiment to verify the ion temperature anisotropy/beta inverse correlation observed by spacecraft in the magnetosphere. Manheimer and Boris ${ }^{1}$ first proposed the idea that a plasma instability threshold derived from linear theory should correspond to an observable bound on the anisotropy driving the unstable mode. Computational simulations ${ }^{2,3}$ of magnetospherically relevant plasmas have been used to interpret the ion temperature anisotropy/beta inverse correlation observed by spacecraft as an upper bound on the ion temperature anisotropy in the magnetosphere. ${ }^{4,5,6}$ If this interpretation were correct, it would support the Manheimer and Boris idea. Previous laboratory experiments have observed electromagnetic ion temperature anisotropy driven

instabilities. ${ }^{7,} 8,9$ However, none have reported observations of an ion temperature anisotropy/beta inverse correlation. Here, measurements clearly show this inverse correlation and that the reduction of the ion temperature anisotropy is correlated with the amplitude of low frequency $\left(0.5 \leq \omega \leq \omega_{c i}\right)$, electromagnetic fluctuations. Measured characteristics of these fluctuations are consistent with those of the ion cyclotron anisotropy instability, also known as the Alfvén ion cyclotron instability. The scaling of the upper bound on the ion temperature anisotropy with plasma beta is also in good agreement with spacecraft observations in the magnetosheath.

The wide range of plasma regimes in the near-Earth space environment provides unparalleled opportunities for testing the predictions of theory and computation. Unfortunately, in space, controlled experiments are rarely possible. Single-point spacecraft observations have provided a wealth of information concerning a variety of instabilities. This information has been used to test existing theories and lay the groundwork for new theories. However, single-point spacecraft measurements cannot perform reproducible experiments and cannot separate spatially varying from temporally varying plasma phenomena. From a moving spacecraft, a spatial variation appears as a temporal variation. In addition, spacecraft cannot measure the wavelengths of instabilities 
much larger than themselves. In order to obtain a wavelength measurement, time series measurements must be measured at a minimum of two different spatial locations.

The Large Experiment on Instabilities and Anisotropies (LEIA) experiment at West Virginia University (WVU) is designed to generate controlled levels of ion temperature anisotropy to investigate ion temperature anisotropy driven instabilities in a fully diagnosed laboratory experiment. Although other laboratory experiments have observed ion temperature anisotropy driven instabilities, those experiments were not intended to address issues relevant to magnetosheath simulations and in-situ space data. The results presented here represent an effort to corroborate laboratory experimental results with computational models and spacecraft observations.

A key element in the interpretation of spacecraft results has been the use of theory and computational simulations. In the case of ion temperature anisotropy driven instabilities, hybrid-kinetic simulations suggest that the inverse correlation between the plasma beta and the ion temperature anisotropy in the magnetosheath arises from velocity space diffusion due to ion cyclotron anisotropy instabilities. The frequencies of and the levels of wave activity seen by the spacecraft are also consistent with the models.

The apparatus used for these experiments is described in Chapter 2. In addition to descriptions of the hardware and the data acquisition system, background information concerning helicon plasma sources and previous results are reviewed. The space simulation chamber is also described in Chapter 2. In Chapter 3, the diagnostic tools used in the space simulation chamber, including Langmuir probes, laser induced fluorescence (LIF) probes, and magnetic probes are discussed.

A brief review of the theory of ion temperature anisotropy driven instabilities is provided in Chapter 4. Although the observed values of ion temperature anisotropy in

LEIA $\left(T_{\perp} / T_{\|}>1\right)$ could permit both the ion cyclotron instability and the mirror instability to exist, the value of ion beta in LEIA $(\beta<1)$ results in the dominance of the ion cyclotron instability. Predictions of the beta-dependant ion temperature anisotropy upper bound from computational models and in-situ spacecraft measurements of both ion temperature anisotropy and wave measurements are also presented in Chapter 4.

Laboratory measurements of the ion temperature anisotropy upper bound are presented and compared to theory and spacecraft observations in Chapter 5. The effects 
of varying the collisionality and the role of low frequency waves reducing the ion temperature anisotropy are also discussed in Chapter 5.

In Chapter 6, the predicted scaling of the amplitude and the characteristics of magnetic fluctuations are compared to measurements in LEIA. In-situ magnetosheath observations of the beta-dependant upper bound on the ion temperature anisotropy without observations of strong wave activity present are reviewed and wavenumbers for the experimental cases studied are also compared to theoretical predictions in Chapter 6. The experimental results are summarized and possible directions for future work are discussed in Chapter 7. 


\section{Plasma Experiment}

The apparatus used in these experiments was designed to provide an environment for performing high beta, magnetospherically relevant plasma experiments. To achieve high values of beta $(\beta>0.01)$, a high-density plasma confined by a low magnetic field is desired. A high-density plasma was created in a helicon plasma source, HELIX (Hot hELIcon eXperiment) (Figure 1), and allowed to expand into LEIA (Large Experiment on Instabilities and Anisotropies), a space simulation chamber (Figure 2). LEIA is a low magnetic field, large volume chamber. Typical ion $\beta$ values for different plasma environments are tabulated in Table 1. A value of 0.01 is considered high ion beta for the purposes of the experiments described here. It is true that magnetosheath beta values often exceed 10 to 100 , but the intention of these experiments is to investigate specific microphysical issues and not to reproduce the magnetosheath exactly.

Table 1: Typical ion beta values for various plasma environments.

\begin{tabular}{|c|c|c|c|c|}
\hline Environment & $n\left(\mathrm{~cm}^{-3}\right)$ & $T_{\mathrm{i}}(\mathrm{eV})$ & $B(\mathbf{G})$ & $\beta_{\mathrm{i}}$ \\
\hline Magnetosheath $^{10}$ & 8 & 150 & $1.5 \times 10^{-4}$ & $\geq 1$ \\
\hline Heliosphere $^{10}$ & 7 & 10 & $7 \times 10^{-5}$ & $\sim 1$ \\
\hline Tokamak (TFTR) $^{11}$ & $10^{14}$ & 32,000 & 56,000 & $4 \times 10^{-2}$ \\
\hline LEIA & $10^{12}$ & 0.3 & 35 & $1 \times 10^{-2}$ \\
\hline $\begin{array}{c}\text { Tokamak }^{12} \\
\text { (Alcator C-MOD) }\end{array}$ & $3 \times 10^{14}$ & 600 & 30,000 & $8 \times 10^{-3}$ \\
\hline HELIX & $10^{14}$ & 0.5 & 500 & $8 \times 10^{-3}$ \\
\hline Ionosphere $^{10}$ & $10^{5}$ & .2 & .26 & $1 \times 10^{-5}$ \\
\hline Q-machine ${ }^{13}$ & $10^{12}$ & .2 & 1000 & $8 \times 10^{-6}$ \\
\hline
\end{tabular}




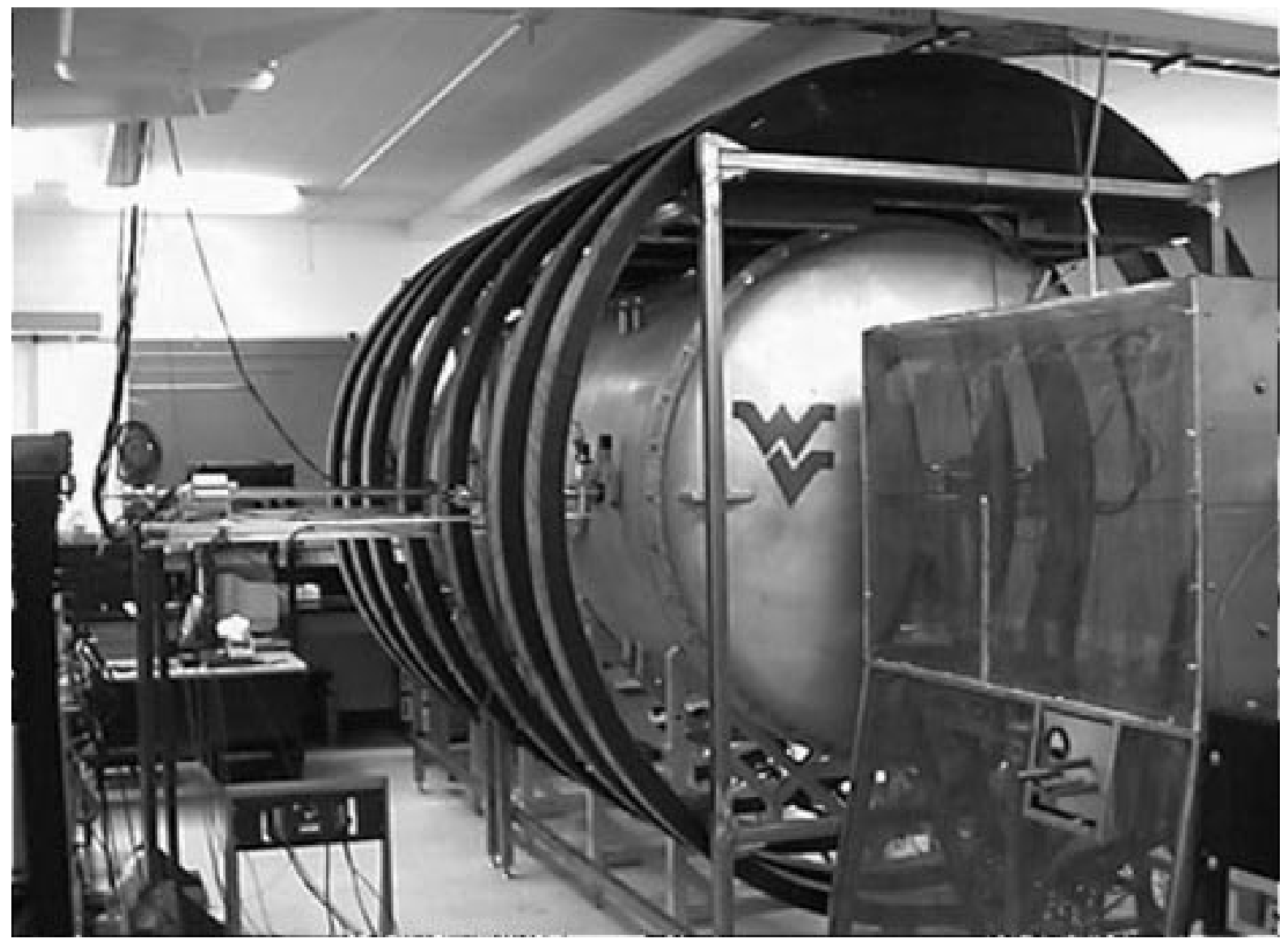

Figure 1: HELIX (foreground) and LEIA (large aluminum chamber). HELIX resides inside a Faraday cage (copper screening). The large electromagnets surrounding LEIA are roughly $3 \mathrm{~m}$ in diameter.

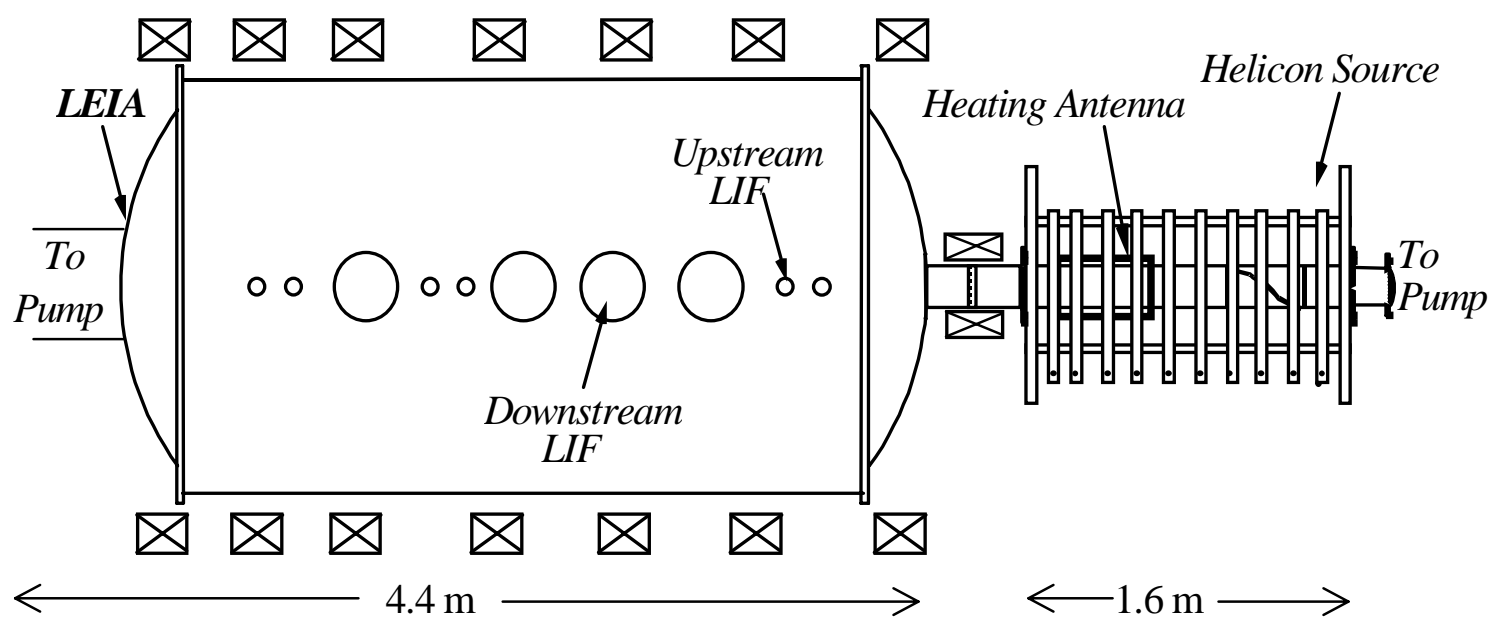

Figure 2: Schematic of LEIA and HELIX. 


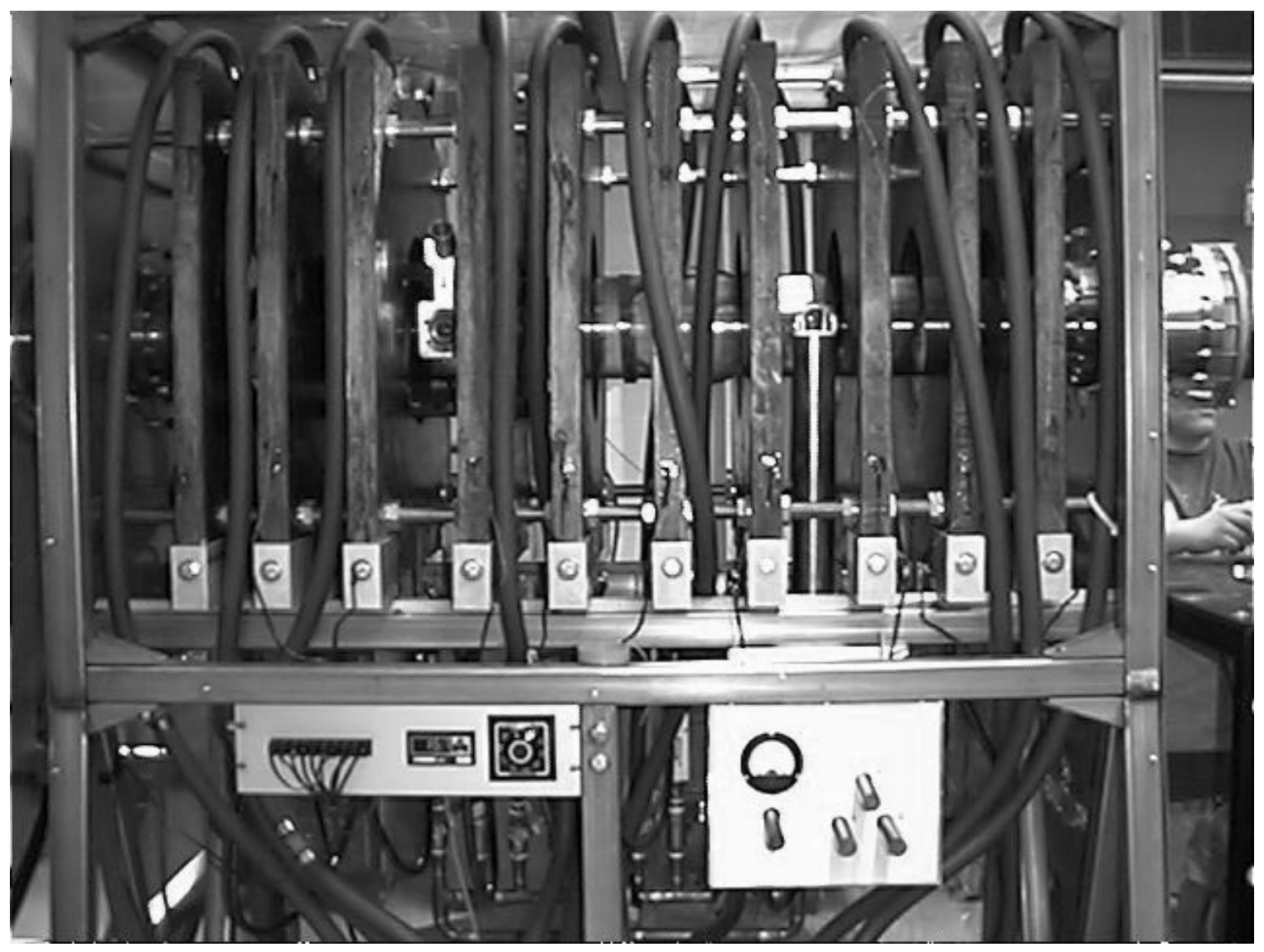

Figure 3: The HELIX plasma source. On the right side is the mating flange to the pumping station. Sitting on the rails are ten electromagnets used to confine the plasma. The matching circuit and the antenna are to the lower right of the picture. At the top of the picture, copper screening, which composes part of the Faraday cage, is visible.

\subsection{Plasma Source}

The helicon source (Figure 3) is a Pyrex tube, $157 \mathrm{~cm}$ long and $15 \mathrm{~cm}$ in diameter. Typical operating parameters for HELIX are listed in Table 2. There are four perpendicular view ports (see Figure 4) to allow optical access to the source. Copper screening around the helicon source forms a Faraday cage. The Faraday cage prevents extraneous high frequency electromagnetic signals from entering the source chamber and prevents the rf driving signal from radiating into the laboratory. At the right-hand side of Figure 3 is a stainless steel mating flange that connects the helicon source to the pumping station. Gas is fed into the chamber through a valve in the mating flange. Both argon and 
helium gas are used, however all of the work presented here used only argon gas. The pumping station consists of a Balzer's TMU 520 turbo molecular pump connected to a Balzer's MD 4T diaphragm roughing pump. The pumps are separated from the glass cross by a MDC GV-4000M-P 6 inch ID (inner diameter) gate valve. At the left end of the source is a stainless steel bellows that connects to the large chamber known as LEIA.

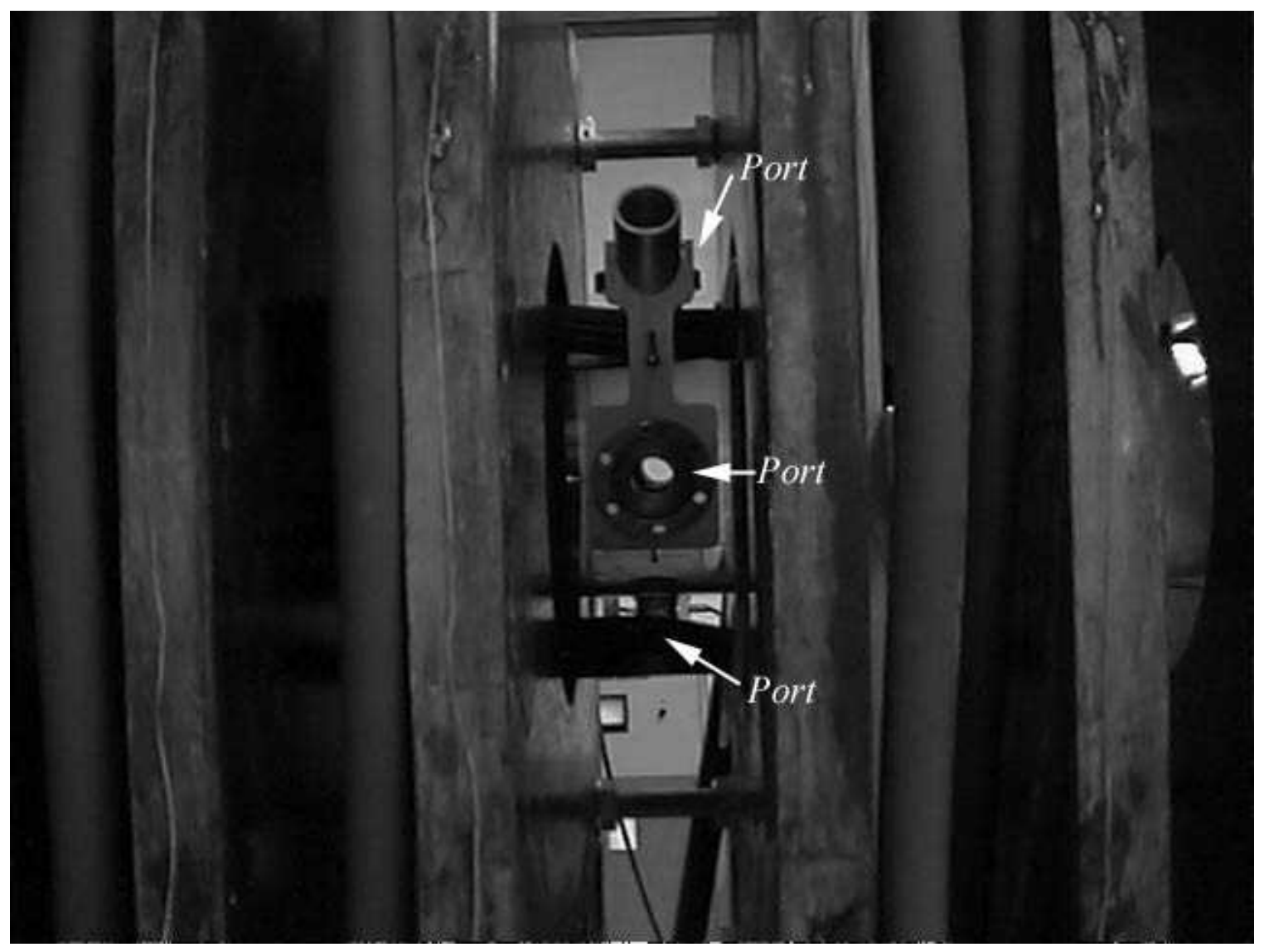

Figure 4: Close-up of the HELIX helicon source showing the four perpendicular view ports between two electromagnets. Also visible is a 45 degree injection optic mounted on a view port. 
Table 2: Standard operating parameters for HELIX

\begin{tabular}{|c|c|}
\hline Parameter & Typical HELIX Values \\
\hline Gas Species & $157 \mathrm{~cm}$ \\
\hline Source Length & $15 \mathrm{~cm}$ \\
\hline Source Radius & $<2 \times 10^{-8}$ Torr \\
\hline Base Pressure & $1-10 \mathrm{mTorr}$ \\
\hline Operating Pressure & $<1300 \mathrm{G}$ \\
\hline Magnetic Field & $0-500 \mathrm{~W}$ \\
\hline RF Power & $7-15 \mathrm{MHz}$ \\
\hline Operating Frequency & $\leq 10^{14} \mathrm{~cm}{ }^{-3}$ \\
\hline Density & $\sim 5 \mathrm{eV}$ \\
\hline Electron Temperature & $<1 \mathrm{eV}$ \\
\hline Ion Temperature & $1-5$ \\
\hline Ion Temperature Anisotropy $\left(T_{\perp} / T_{\|}\right)$ & $\sim .03 \mathrm{~mm}$ \\
\hline Electron Gyroradius & $\sim .12 \mathrm{~mm}$ \\
\hline Ion Gyroradius & \\
\hline
\end{tabular}

The magnetic field for the helicon source is created by ten electromagnets donated by the Max Planck Institüt in Garching, Germany. The magnets have 46 internal copper windings with a resistance of $17 \mathrm{~m} \Omega$ and an inductance of $1.2 \mathrm{mH}$. The magnets are water cooled to prevent overheating. The magnets roll on a pair of rails that allow their axial positions to be adjusted. A Macroamp 300 A power supply provides the current for the electromagnets. The magnetic field in HELIX can be varied from 0 to 1300 Gauss. The on-axis magnetic field in HELIX is given by

$$
B=6.08+3.93 I,
$$

where $I$ is the coil current. This relationship was determined experimentally by varying the current supplied to the magnets and measuring the magnetic field with a gaussmeter. 
Presently, the second magnet from the end of the source closest to the pumping station has been reversed to create a field null. The field null was used to isolate the pumping station from the plasma and because other helicon source groups have reported increased densities using field nulls near one end of the helicon antenna. ${ }^{14}$

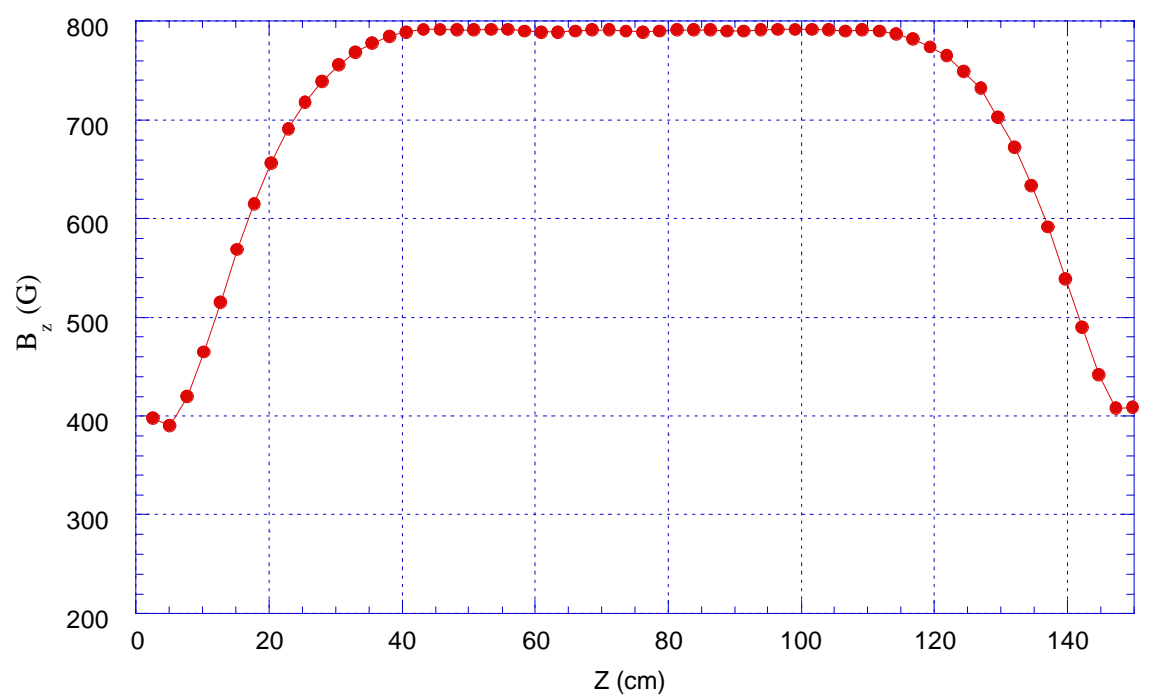

Figure 5: Magnetic field strength as a function of axial position $(z)$ in HELIX.

The azimuthal and radial $B_{z}$ magnetic field profiles in HELIX are shown in Figure 5 and Figure 6 . The radial magnetic field is uniform to within $1 \%$ over the chamber radius and uniform to within $5 \%$ along the central meter of the chamber. 


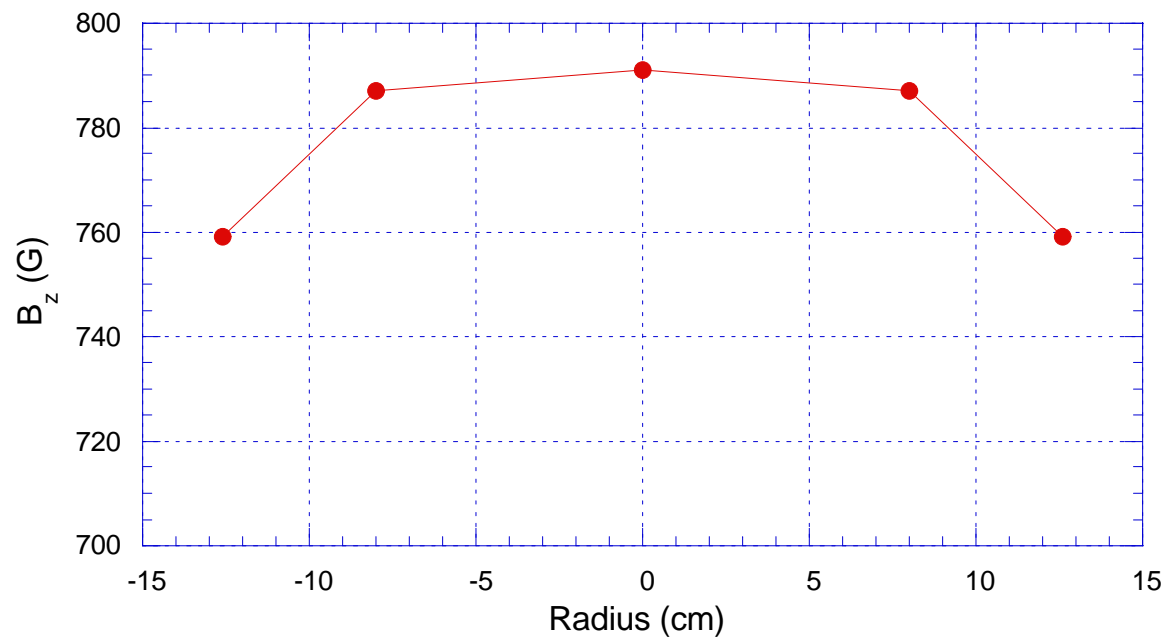

Figure 6: Magnetic field in HELIX as a function of radial position. Note the suppressed zero in the y-axis scale.

In order to create the plasma, an rf signal is applied to a copper antenna wrapped around the outside of the source chamber. A $50 \mathrm{MHz}$ Wavetek function generator supplies a rf signal to an ENI 2000 amplifier that has a frequency range of $0.3-35 \mathrm{MHz}$ and can provide up to $2 \mathrm{~kW}$ of $\mathrm{rf}$ power. The amplifier output is coupled to the antenna through a pi-matching network (Figure 7). The matching network is needed because the amplifier's output impedance is $50 \mathrm{Ohms}$ and the real impedance of the antenna is much smaller than 50 ohms. The matching network consists of one load and three tuning capacitors. The load capacitor is a Jennings high voltage tunable (20-2000 pF) capacitor. The three tuning capacitors are all Jennings high voltage tunable capacitors, two of which have a range of $4-250 \mathrm{pF}$ and the third has a range of $5-500 \mathrm{pF}$. The three tuning capacitors are in parallel with each other and in series with the antenna. This combination is then all in parallel with the load capacitor.

Francis Chen's internal University of California at Los Angeles (UCLA) report describes how to design a capacitive circuit to match to an inductive load. ${ }^{15} \mathrm{He}$ considered two types of circuits, the "standard circuit" and the "alternative circuit." His discussion of the standard, pi, circuit is reviewed here. 


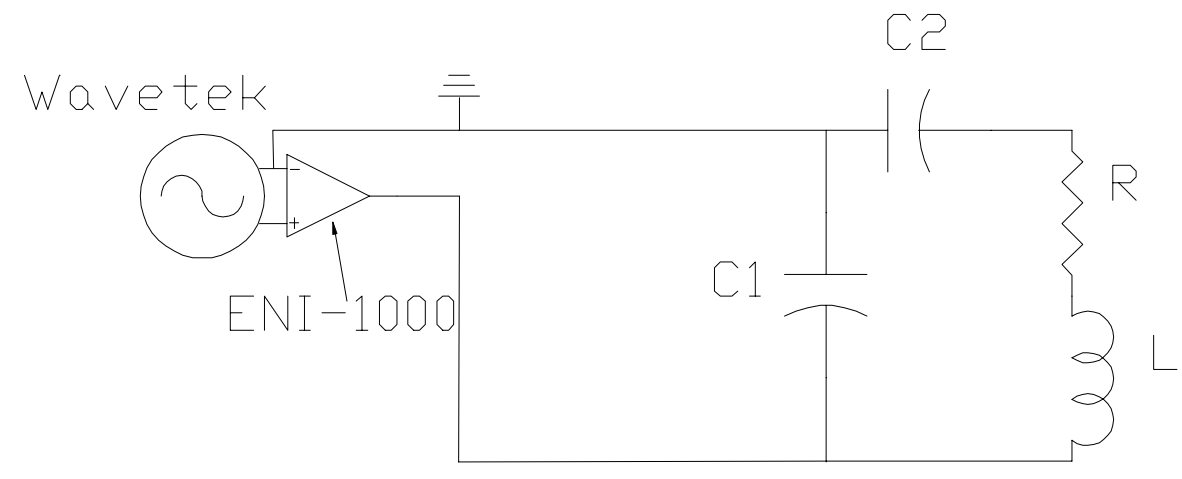

Figure 7: Schematic of the standard circuit. C1 is the load capacitor, C2 is the tuning capacitors and $\mathrm{R}+\mathrm{L}$ comprise the real resistance and inductance of the antenna.

In Chen's analysis, all the impedances are normalized to $R_{0}=50 \Omega$. Thus, to maximize the efficiency of the circuit, the circuit elements are chosen such that the real part of the impedance is 1 and the imaginary part is 0 . In terms of the impedance of the combined load and matching circuit system, the input impedance $(Z)$ is.

$$
Z=\left(Z_{1}^{-1}+Z_{2}^{-1}\right)^{-1}
$$

where,

$$
Z_{1}=-\frac{i}{\omega C_{1}}, \text { and } Z_{2}=R+i X-\frac{i}{\omega C_{2}} .
$$

$R$ is the real resistance of the antenna and $X$ is the reactance of the antenna. $C_{1}$ is the load capacitor and $C_{2}$ is the tuning capacitor. Substituting these expressions into equation (2),

$$
Z=\frac{R+i\left(X-1 / \omega C_{2}\right)}{1-\omega C_{1}\left(X-i / \omega C_{2}\right)+i \omega R C_{1}}=\frac{(R+i Q)\left(1-\omega C_{1} Q-i \omega R C_{1}\right)}{\left(1-\omega C_{1} Q\right)^{2}+\omega^{2} R^{2} C_{1}^{2}},
$$

where

$$
Q \equiv X-1 / \omega C_{2} .
$$

Defining

$$
D \equiv\left(1-\omega C_{1} Q\right)^{2}+\omega^{2} R^{2} C_{1}^{2},
$$


the impedance can be separated into real and imaginary parts and written as

$$
\begin{gathered}
D \operatorname{Re}(Z)=R\left(1-\omega C_{1} Q\right)+\omega R C_{1} Q \\
D \operatorname{Im}(Z)=Q\left(1-\omega C_{1} Q\right)-R^{2} C_{1}
\end{gathered}
$$

which reduces to

$$
D \operatorname{Re}(Z)=R
$$

and

$$
D \operatorname{Im}(Z)=Q-\omega C_{1}\left(R^{2}+Q^{2}\right) .
$$

Requiring $\operatorname{Im}(Z)=0$ yields,

$$
Q=\omega C_{1}\left(R^{2}+Q^{2}\right) .
$$

Substituting this into equation (6):

$$
D=1-\omega C_{1} Q .
$$

Setting $\operatorname{Re}(Z)=1$ requires:

$$
R /\left(1-\omega C_{1} Q\right)=1
$$

and solving the quadratic for $Q$ yields:

$$
\omega C_{1}=(1-R) / Q .
$$

Rewriting equation (11), $Q$ can be written as a quadratic

$$
\omega C_{1} Q^{2}-Q+\omega C_{1} R^{2}=0 .
$$

Thus,

$$
2 \omega C_{1} Q=1 \pm\left(1-4 \omega^{2} C_{1}^{2} R^{2}\right)^{1 / 2} .
$$

Combining equations (14) and (16),

$$
1-2 R= \pm\left(1-4 \omega^{2} C_{1}^{2} R^{2}\right)^{1 / 2} .
$$

With this result it is possible to determine the load capacitance necessary to match the circuit independent of the antenna reactance. Squaring both sides and solving for $C_{1}$

$$
\begin{gathered}
(1-2 R)^{2}=1-4 \omega^{2} C_{1}^{2} R^{2} \\
C_{1}=\left[1-(1-2 R)^{2}\right]^{1 / 2} / 2 \omega R .
\end{gathered}
$$


Using this value for $C_{1}, C_{2}$ can be determined as a function of $C_{1}, R, \omega$, and $X$.

Combining equations (5) and (14) yields

$$
\omega C_{2}=\left(X-\frac{1-R}{\omega C_{1}}\right)^{-1} .
$$

The solutions for $C_{1}$ and $C_{2}$ can be rewritten as

$$
\begin{gathered}
C_{1}=\frac{1}{2 \omega R}\left[1-\left(1-\frac{2 R}{R_{o}}\right)^{2}\right]^{\frac{1}{2}} \\
C_{2}=\left[\omega X-\frac{1-R / R_{o}}{C_{1}}\right]^{-1} .
\end{gathered}
$$

For a purely inductive load $(X=\omega L)$, such as is expected for the HELIX configuration,

$$
C_{2}^{-1}=\omega^{2} L-\left(1-R / R_{o}\right) / C_{1} .
$$

Solutions for $C_{1}$ and $C_{2}$ for a purely inductive load are graphed in Figure 8 and Figure 9 respectively. If $L<\left(1-R / R_{o}\right) / \omega^{2} C_{1}$, there is no positive solution for $C_{2}$. For the typical case of $R / R_{o}<<1$, equations (21) and (22) can be approximated by

$$
\omega C_{1} \approx\left(R R_{o}\right)^{-\frac{1}{2}} \text { and } \omega C_{2} \approx\left[X-\left(R R_{o}\right)^{1 / 2}\right]^{-1} \text {. }
$$

\section{Loading capacitor for standard circuit}

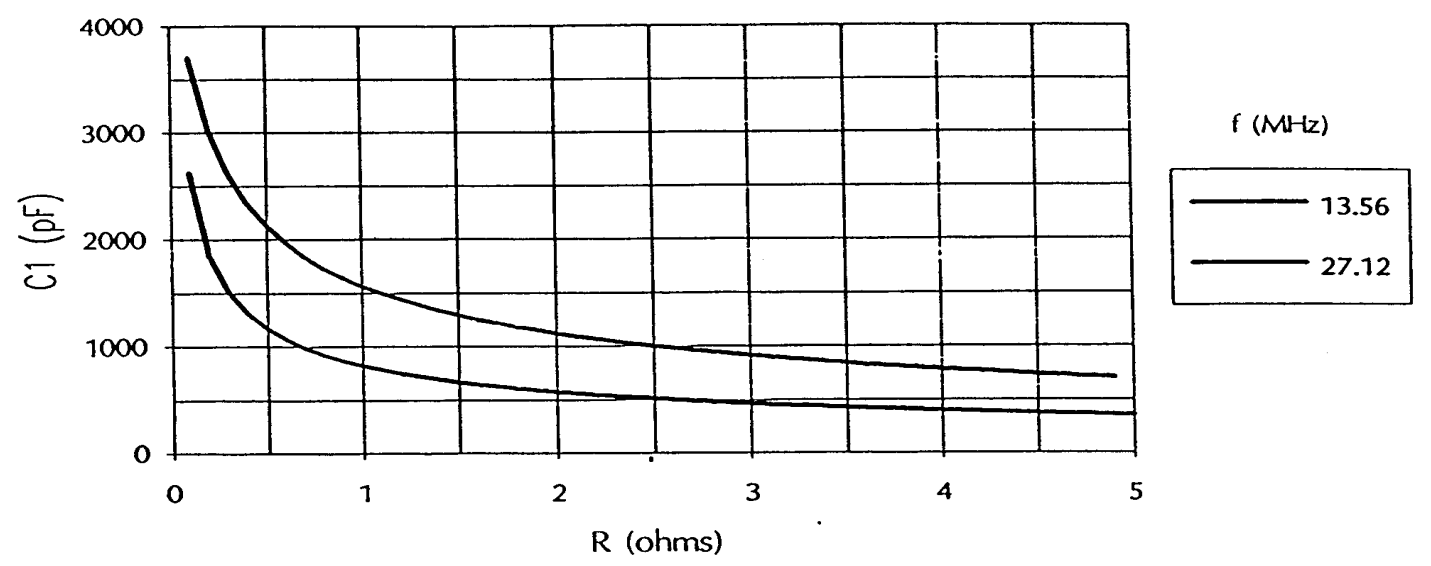

Figure 8: Relationship between the capacitance and inductance of the load capacitor in a matching circuit for 13.56 (top curve) and 27.12 (bottom curve) $\mathrm{MHz}$ as shown by Chen. ${ }^{15}$ 
Tuning capacitor for standard circuit

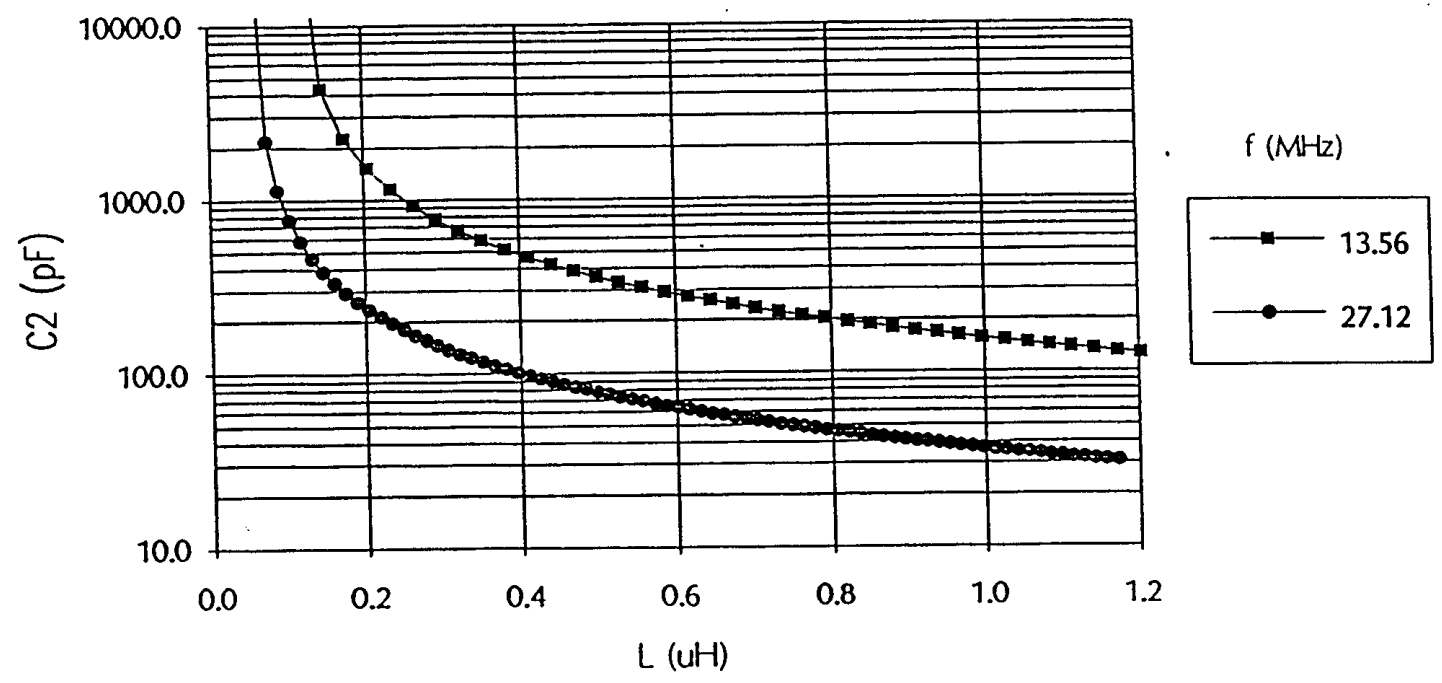

Figure 9: Capacitance versus inductance for the tuning capacitor. Note that for 13.56 $\mathrm{MHz}$, it is impossible to match an inductance of less than $0.15 \mu \mathrm{H}$. As the frequency increases, smaller inductances can be matched with the standard circuit. ${ }^{15}$

Though every helicon source varies in its design, some sort of antenna is needed to launch the helicon wave. Some of the more popular antennas studied by various groups are the Boswell saddle antenna, the Nagoya Type III antenna and the helical (right and left) antennas. Initial characterization of HELIX was accomplished using a Nagoya Type III antenna. ${ }^{37}$ Current experiments employ a right helical $(m=+1)$ antenna. In order to reduce resistive losses in the matching network due to coaxial cables, the matching network was redesigned to use copper bars for transmission instead of coaxial cable. ${ }^{16} \mathrm{~A}$ recent investigation of antenna efficiency examined the relative effectiveness of a Boswell saddle, Nagoya type III, and a right helical antenna. A long, right helical antenna yielded the highest densities and also appears to increase the perpendicular ion temperature compared to a shorter right helical antenna. ${ }^{16}$ In all cases, right helical antennas generated higher densities then Boswell saddle or Nagoya type III antennas. ${ }^{16}$ The antenna used for the experiments reported in this thesis was a $19 \mathrm{~cm}$ long, right helical antenna. 
HELIX is different from most helicon sources because it is operated steady-state. Running steady-state allows for the same plasma environment to be studied during a series of measurements. However, probes placed in the plasma degrade much faster than in a pulsed source. This increases the level of contaminants in the system. To counteract this, all measurements performed in HELIX use non-invasive techniques.

The ion-heating antenna is another unique feature of HELIX. The antenna, shown in Figure 1, is used to heat the ions in the source to increase the level of ion temperature anisotropy. ${ }^{17}$ Some of the parameters of operational helicon sources are compared in Table 3.

Table 3: Comparison of helicon sources and their typical running parameters.

\begin{tabular}{|c|c|c|c|c|c|c|}
\hline Parameters & $\begin{array}{l}\text { HELIX } \\
\text { (WVU) }\end{array}$ & $\begin{array}{l}\text { Degeling } \\
\text { et } a l .^{54} \\
(\mathrm{ANU})\end{array}$ & $\begin{array}{l}\text { Light } e t \\
\quad a l^{18} \\
\text { (UCLA) }\end{array}$ & $\begin{array}{l}\text { Guo et al } \\
\text { (UW-Mad) }\end{array}$ & $\begin{array}{l}\text { Shoji et } \\
a l^{20} \\
\text { (Japan ) }\end{array}$ & $\begin{array}{c}\text { Yun et } \\
a^{46}{ }^{46} \\
\text { (Korea) }\end{array}$ \\
\hline Power & $\begin{array}{c}\text { Steady } \\
\text { State }\end{array}$ & Pulsed & Pulsed & Pulsed & Pulsed & $\begin{array}{l}\text { Steady } \\
\text { State }\end{array}$ \\
\hline Diameter $(\mathbf{c m})$ & 15.2 & 18 & 2.5 & 10 & 5 & 25 \\
\hline Length (cm) & 157 & 50 & 170 & 122 & 50 & 60 \\
\hline Aspect Ratio (r/L) & .049 & .18 & .0075 & .041 & .05 & .21 \\
\hline Gases & $\mathrm{Ar}, \mathrm{He}$ & $\mathrm{Ar}$ & $\mathrm{Ar}$ & Ar, N & $\mathrm{Ar}$ & $\mathrm{Ar}$ \\
\hline $\begin{array}{c}\text { Driving Freq } \\
\text { (MHz) }\end{array}$ & $8-15$ & 13.56 & 27.12 & 13.56 & 7 & $1-8$ \\
\hline Mag. Field (G) & 1300 & 50 & 900 & 1500 & 3000 & $<1500$ \\
\hline Densities $\left(\mathrm{cm}^{-3}\right)$ & $<10^{14}$ & $<5 \times 10^{11}$ & $<3 \times 10^{13}$ & $<2 \times 10^{13}$ & $<3 \times 10^{13}$ & $<8 \times 10^{12}$ \\
\hline$\omega_{L H}(\mathbf{M H z})$ & $<33$ & 1.3 & $<19$ & $<39$ & $<78$ & $<39$ \\
\hline$\omega_{c e}(\mathbf{G H z})$ & $<3.6$ & $\sim .14$ & $<1.6$ & $<4.2$ & $<8.4$ & $<8.4$ \\
\hline
\end{tabular}




\subsection{Helicon Sources}

The helicon plasma source is a high-density, highly efficient, inductively coupled plasma source that was first described in 1970 by Boswell. ${ }^{21}$ The helicon plasma source has been used as a source for materials processing, ${ }^{22,23,24,25,26}$ for magnetic fusion ${ }^{27}$ (Heliac), for high beta, space simulation experiments, and for space plasma thruster development. ${ }^{28}$ Many groups have attempted to explain the mechanism for the efficient ionization of helicon sources by Landau damping, energetic beams of electrons, and Trivelpiece-Gould (TG) waves. At present, a definitive answer has not been obtained.

\subsubsection{Background}

The term "helicon" was first used by Aigrain ${ }^{29}$ in 1960 to describe an electromagnetic wave propagating in a low temperature solid metal with a frequency between the ion and electron cyclotron frequencies, i.e., a whistler wave. Around 1964, Legendy ${ }^{30}$ in the United States and Klosenberg, McNamara and Thonemann ${ }^{31}$ in the United Kingdom developed theories concerning the propagation of helicon waves in cylindrical magnetoplasmas. In 1968, Boswell constructed a helicon experiment at Flinders University using what is now known as the Boswell saddle antenna (Figure 10). ${ }^{32}$ In that experiment, Boswell measured the density as a function of pressure (Figure 11) and the azimuthal component of the magnetic field, $B_{z}$ (Figure 12). Boswell observed resonances in $B_{z}$ that changed when the pressure or magnetic field was changed. Boswell also noticed a density maximum at a field between $400 \mathrm{G}$ and $1150 \mathrm{G}$. Using a $35 \mathrm{GHz}$ interferometer, he estimated this density to be $3 \times 10^{13} \mathrm{~cm}^{-3}$. The plasma color was a blue "too bright to observe directly with the eye." ${ }^{33}$ This blue is indicative of the commonly seen "helicon core," where the helicon wave propagates and higher densities are found. Boswell's core had a diameter of approximately $1 \mathrm{~cm}$ and extended past his last magnet until it reached the glass boundary of his experiment. The glass became so hot that it melted within a minute or two. Boswell then constructed a larger source, $10 \mathrm{~cm}$ diameter and $120 \mathrm{~cm}$ long and observed similar results to the smaller helicon source. ${ }^{21}$ 


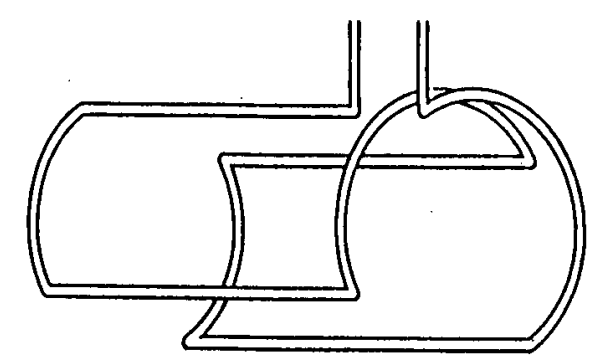

Figure 10: Schematic of the Boswell saddle antenna. ${ }^{\mathbf{3 4}}$

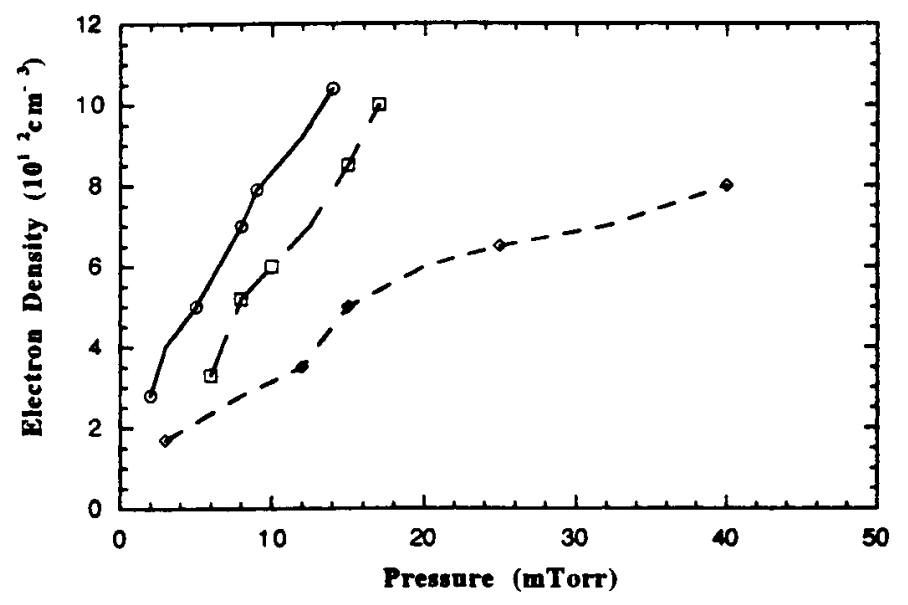

Figure 11: Density as a function of pressure for three different magnetic fields (1150 G solid line, $2000 \mathrm{G}$ - large dashes, $400 \mathrm{G}$ - small dashes) from Boswell's first experiment.

Note that the highest densities do not correspond to the highest magnetic fields. ${ }^{33}$ 


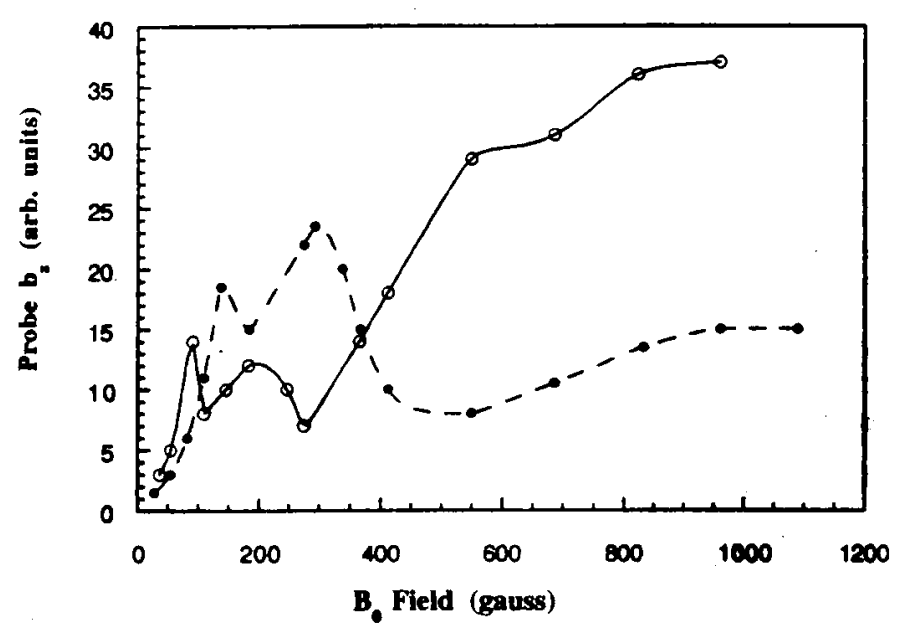

Figure 12: Measured $B_{z}$ for $9 \mathrm{mTorr}$ (solid line) and $27 \mathrm{mTorr}$ (dashed line). Resonances in $B_{z}$ appear to change with different pressures. ${ }^{33}$

Boswell was one of the first researchers to report "jumps" in the helicon source density. ${ }^{35}$ Physically, these jumps correspond to an increase in plasma brightness (density). Figure 13 shows Boswell's measurements of the electron density versus magnetic field in an early helicon source. Even though the general trend of the data is well fit with a straight line corresponding to the whistler dispersion relationship, the plasma density is roughly constant across small ranges of magnetic field. Between the constant density regions, there is a sudden transition to another density value as the magnetic field is increased. These jumps occur when there is a mode transition in the helicon source. Three modes of operation have been identified, the capacitive, the inductive and the helicon mode. 


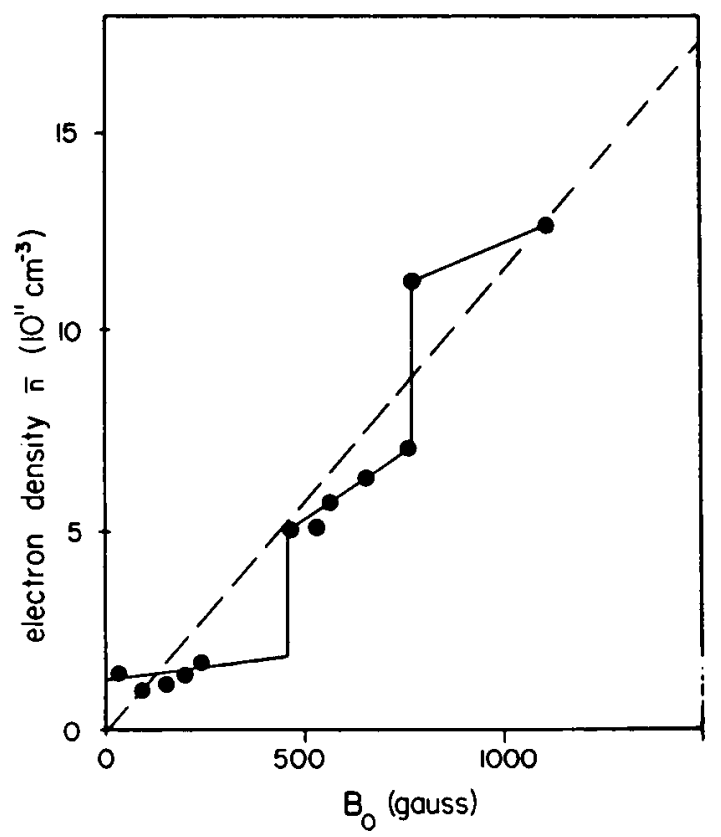

Figure 13: Results from Boswell showing the helicon source density as a function of magnetic field. The data has "jumps" which indicate different modes of operation. The dashed line is the expected relationship from the simple whistler wave dispersion relation. $^{35}$

These modes have been observed by most helicon groups and have been studied extensively by Ellingboe and Boswell. ${ }^{36}$ They introduced the terminology of E-mode for the capacitive mode, $\mathrm{H}$-mode for the inductive mode and $\mathrm{W}$-mode for the helicon mode. Between these three modes are two jumps, at the E-H mode transition and at the $\mathrm{H}-\mathrm{W}$ mode transition, where the plasma-wave-field profiles change dramatically. Figure 14 shows HELIX data that demonstrates the different mode transitions. 


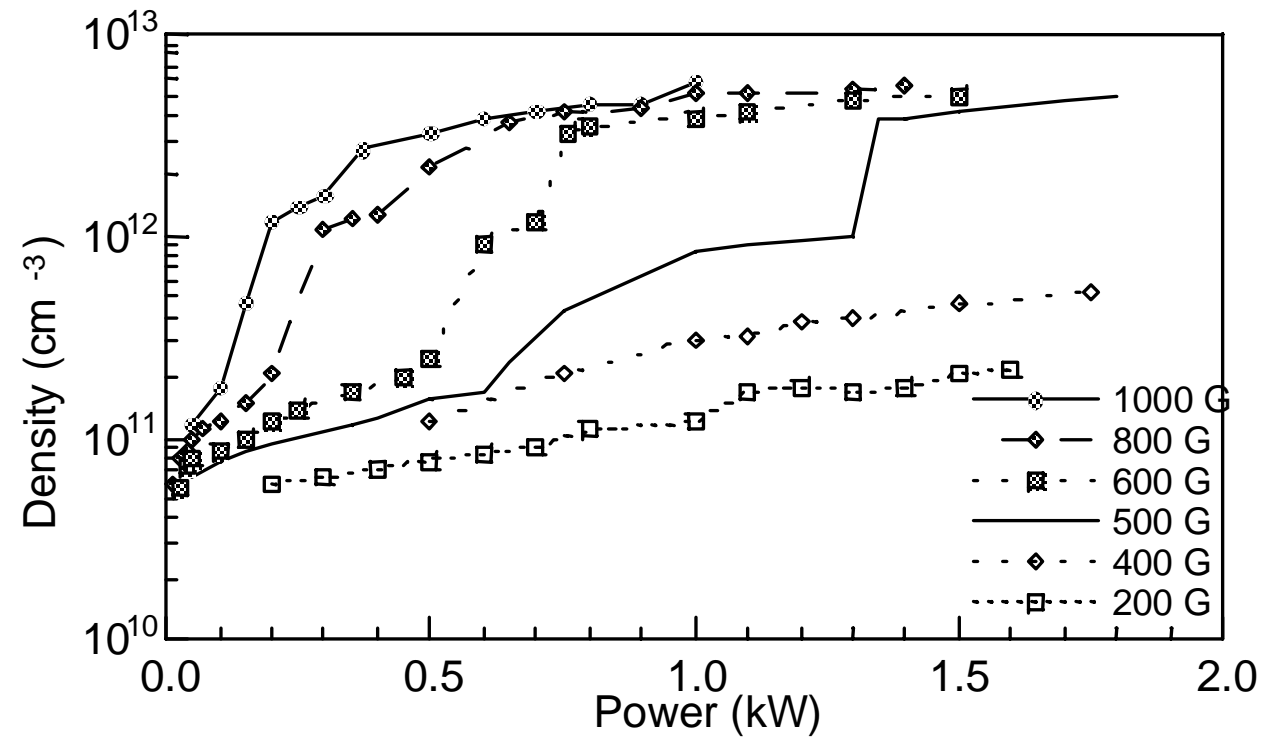

Figure 14: Density as a function of rf power for six different magnetic fields from HELIX. ${ }^{37}$ Note the jumps in density, which represent different modes of operation.

The E-H transition in HELIX occurs at a density of roughly $10^{11} \mathrm{~cm}^{-3}$ regardless of the magnetic field or pressure. This transition is related to the electron skin depth, $\delta=c / \omega_{p e}$, where $\omega_{p e}=\left(4 \pi n_{e} e^{2} / m_{e}\right)^{1 / 2}$ is the electron plasma frequency. The E-H transition generally occurs when $\delta$ is approximately half the chamber diameter.

There is a well-documented change in the density profile at the $\mathrm{H}-\mathrm{W}$ transition. In the $\mathrm{H}$ mode, the profiles are often observed to be hollow, while the profiles are much more centrally peaked in the $\mathrm{W}$ mode. Traveling waves become observable in the $\mathrm{W}$ mode, as opposed to the standing waves associated with the $\mathrm{E}$ and $\mathrm{H}$ modes. As shown in Figure 14 , the $\mathrm{H}$ mode often exists over a very small range of parameters and can often go unnoticed. Recently, the helicon community has shifted its focus from discussion of the $\mathrm{E}, \mathrm{H}$ and $\mathrm{W}$ modes to investigations of the relationship between Trivelpiece-Gould modes and helicon modes. As mentioned previously, the $\mathrm{W}$ and helicon modes are equivalent. The Trivelpiece-Gould mode essentially corresponds to the capacitive, E, mode.

A key researcher in the development of helicon source theory in the 1990's has been Francis Chen at UCLA. In 1985, Chen visited Boswell's group at Australian National 
University (ANU) and began constructing helicon sources upon his return to UCLA. It should be noted that even though Chen's theory work helped lay the groundwork for more interest and understanding of helicon sources, his studies were limited to small aspect ratio sources (long tubes with small radii). In his theory work, Chen also severely restricted the frequency regime by requiring $\omega_{c i}, \omega_{L H}<<\omega<\omega_{c e}, \omega_{p e}$ (where $\omega_{c i}=Z e B / m_{i} c$ is the ion cyclotron frequency, $\omega_{c e}=e B / m_{e} c$ is the electron cyclotron frequency, and $\omega_{L H} \approx \sqrt{\omega_{c e} \omega_{c i}}$ is the lower hybrid frequency) in the source. This definition is more restrictive than the one used by Aigrain $\left(\omega_{c i}<\omega<\omega_{c e}\right)$. Initially, Chen used a Nagoya Type III antenna (Figure 15). At the same time in Japan, Shoji, who learned of the helicon source from Chen, began developing a helicon source. Shoji was the first to employ helical (right or left-handed polarization) antennas ${ }^{38}$ (Figure 16). Helical antennas preferentially launch right $(m=+1)$ or left $(m=-1)$ handed polarized waves.

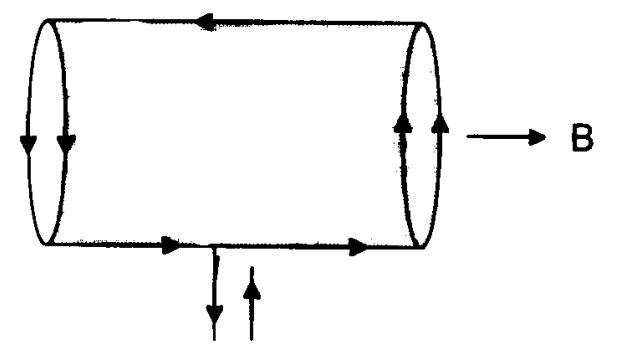

Figure 15: Diagram of a typical Nagoya Type III antenna. ${ }^{39}$

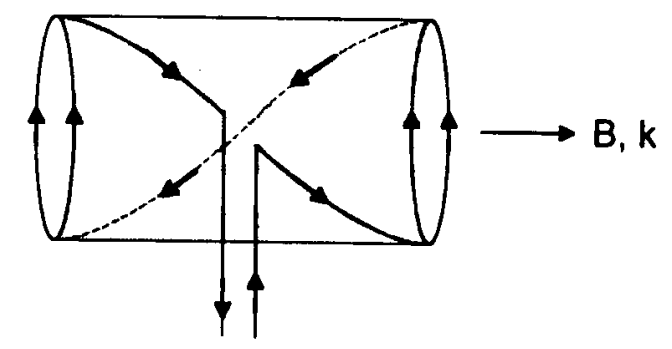

Figure 16: A diagram of a right-helical antenna. ${ }^{39}$ 
Chevalier and Chen ${ }^{14}$ were the first to notice an increase in the plasma density when a field null was located near the antenna. They observed that the density profile became much more peaked compared to conifurations without a field null and that the integrated line density was also larger in the case of the field null. Similar results, including measurements of an increase in the total volume averaged kinetic energy density, have been reported by other helicon groups. ${ }^{40}$

The helicon source has been studied as a possible plasma source for etching of wafers and helicon sources are now available commercially. Over the last five years, the helicon source has also become a popular source for basic plasma experiments. Groups that have recently constructed helicon sources can be found at: Ernst-Moritz-Arndt Univerisität, ${ }^{41}$ University of California at San Diego (UCSD), Auburn University, ${ }^{42}$ Oak Ridge National Laboratory, University of Wisconsin-Madison (UW-Madison), Los Alamos National Laboratory (LANL), Princeton Plasma Physics Laboratory (PPPL), and WVU.

\subsubsection{Helicon Dispersion Relation}

The helicon wave is a right-handed, circularly polarized, electromagnetic wave bounded by an insulating cylinder. The helicon is essentially a whistler wave at a frequency lower than "traditional" whistler waves. Using the cold plasma dielectric tensor solution for the right hand, circularly polarized wave, or $R$ wave, a dispersion relation can be obtained for unbounded plasma waves in the helicon frequency regime. Starting with Maxwell's time-dependent equations and assuming no external currents

$$
\begin{gathered}
\vec{\nabla} \times \vec{E}=i \omega \mu_{o} \vec{H} \\
\vec{\nabla} \times \vec{H}=-i \omega \varepsilon_{o} \vec{K} \cdot \vec{E},
\end{gathered}
$$

where $\vec{K}$ is the dielectric tensor which includes both plasma and displacement currents. $\vec{K}$ is defined as

$$
\vec{K}=\left[\begin{array}{ccc}
S & -i D & 0 \\
i D & S & 0 \\
0 & 0 & P
\end{array}\right]
$$


where the matrix elements are:

$$
\begin{gathered}
S=\frac{1}{2}(R+L) \\
D=\frac{1}{2}(R-L) \\
R \equiv 1-\sum_{s} \frac{\omega_{p s}^{2}}{\omega\left(\omega+\omega_{c s}\right)} \\
L \equiv 1-\sum_{s} \frac{\omega_{p s}^{2}}{\omega\left(\omega-\omega_{c s}\right)} \\
P \equiv 1-\sum \frac{\omega_{p s}^{2}}{\omega^{2}}
\end{gathered}
$$

Taking the curl of equation. (25), performing Fourier analysis $\left(\vec{E}=\vec{E}_{o} e^{i(\vec{k} \cdot \vec{x}-\omega t}\right)$ to obtain the wave equation, and substituting equation (25) into equation (26) yields,

$$
\vec{n} \times(\vec{n} \times \vec{E})+\frac{\omega^{2}}{c^{2}} \vec{K} \cdot \vec{E}=0,
$$

where $\vec{n}=\frac{\vec{k} c}{\omega}$ is the index of refraction and the wave electric field is common in both terms.

The non-trivial solution to equation (33) requires a solution for arbitrary electric fields. Writing the solution in terms of the parallel and perpendicular refractive indices, $\vec{n}_{\|}=\vec{k}_{\| \perp} c / \omega$ and $\vec{n}_{\perp}=\vec{k}_{\perp} c / \omega$ yields,

$$
A n_{\perp}^{4}-B n_{\perp}^{2}+C=0
$$

where

$$
\begin{gathered}
A=S, \\
B=n_{\|}^{2}[(S+P)-(R L+P S)], \\
C=P\left(n_{\|}^{2}-R\right)\left(n_{\|}^{2}-L\right) .
\end{gathered}
$$

The two right circularly polarized solutions to equation (34) are known as the fast (small $k_{\perp}$, high phase velocity, electromagnetic helicon mode) and the slow (large $k_{\perp}$, slow phase velocity, electrostatic Trivelpiece-Gould (TG) mode). For an $R$ wave, which is a right-hand circularly polarized wave, the complete dispersion relation, including collisions, can be written as ${ }^{43}$ 


$$
n^{2}=\frac{c^{2} k^{2}}{\omega^{2}}=1-\frac{\omega_{p e}}{\omega\left(\omega+i \nu-\omega_{c e} \cos \theta\right)},
$$

where

$$
\begin{aligned}
& n^{2}=n_{\|}^{2}+n_{\perp}^{2} \\
& \cos \theta=k_{z} / k, \\
& k^{2}=k_{\|}^{2}+k_{\perp}^{2} .
\end{aligned}
$$

For the frequency regime of the helicon wave, $\omega_{c i}<<\omega<<\omega_{c e}<<\omega_{p e}$, the dispersion relation simplifies to

$$
k^{2}=\frac{\omega \omega_{p e}}{c^{2}\left(\omega_{c e} \cos \theta-\omega-i v\right)} .
$$

Solving for $k$ yields,

$$
k=\frac{\omega_{c e} k_{\|} \pm\left(\omega_{c e}^{2} k_{\|}^{2}-\frac{4 \omega(\omega+i v) \omega_{p e}^{2}}{c^{2}}\right)^{1 / 2}}{2(\omega+i v)}
$$

The two solutions for $k$ correspond to the helicon mode (minus sign) and the TG mode (positive sign). ${ }^{44}$ Rewriting the dispersion relation in terms of frequency leads to two solutions:

$$
\begin{gathered}
\omega=\frac{\omega_{c e} k_{\|} k c^{2}}{\omega_{p e}^{2}}-i v \frac{k^{2} c^{2}}{\omega_{p e}^{2}} \quad \text { (helicon mode), } \\
\omega=\omega_{c e} \frac{k_{\|}}{k}-i v \quad(\text { TG mode }) .
\end{gathered}
$$

For $\omega<<\omega_{c e}$, only the helicon mode exists. The wave at these frequencies is primarily electromagnetic, and this is the parameter regime in which most helicon sources operate. As the frequency approaches the electron cyclotron frequency, the wave becomes more electrostatic and the TG mode dominates. TG modes will be discussed in more detail later in this section.

Assuming there are no collisions, equation (43) reduces to 


$$
\omega=\frac{\omega_{c e} k_{\|} k c^{2}}{\omega_{p e}^{2}} .
$$

Equation (45) is often referred to as the simple helicon dispersion relation. In Chen's notation, for small aspect ratio helicon sources $\left(k_{\perp}^{2}>>k_{\|}^{2}\right)$ the simple dispersion relation is given by ${ }^{45}$

$$
\omega=\frac{\omega_{c e} \alpha k c^{2}}{\omega_{p e}^{2}}
$$

where $k$ corresponds to $k_{\|}$and $\alpha$ corresponds to $k$ in equation (45). Equation (45) indicates that the plasma density in a helicon source should be proportional to the magnetic field and inversely proportional to the antenna driving frequency. As mentioned previously, the density in a helicon source is observed to be roughly linear with the magnetic field ${ }^{35}$ (see Figure 13), though some groups have seen a leveling-off of the density at higher values of the magnetic field (see Figure 18). ${ }^{37,46,47}$ The plasma density in HELIX was observed to obey the inverse scaling with driving frequency, as predicted by equation (45) (Figure 17). ${ }^{37}$ However, other helicon groups, using a broader frequency range, have seen peaks in the density at certain driving frequencies that they attribute to lower hybrid resonances. ${ }^{46}$

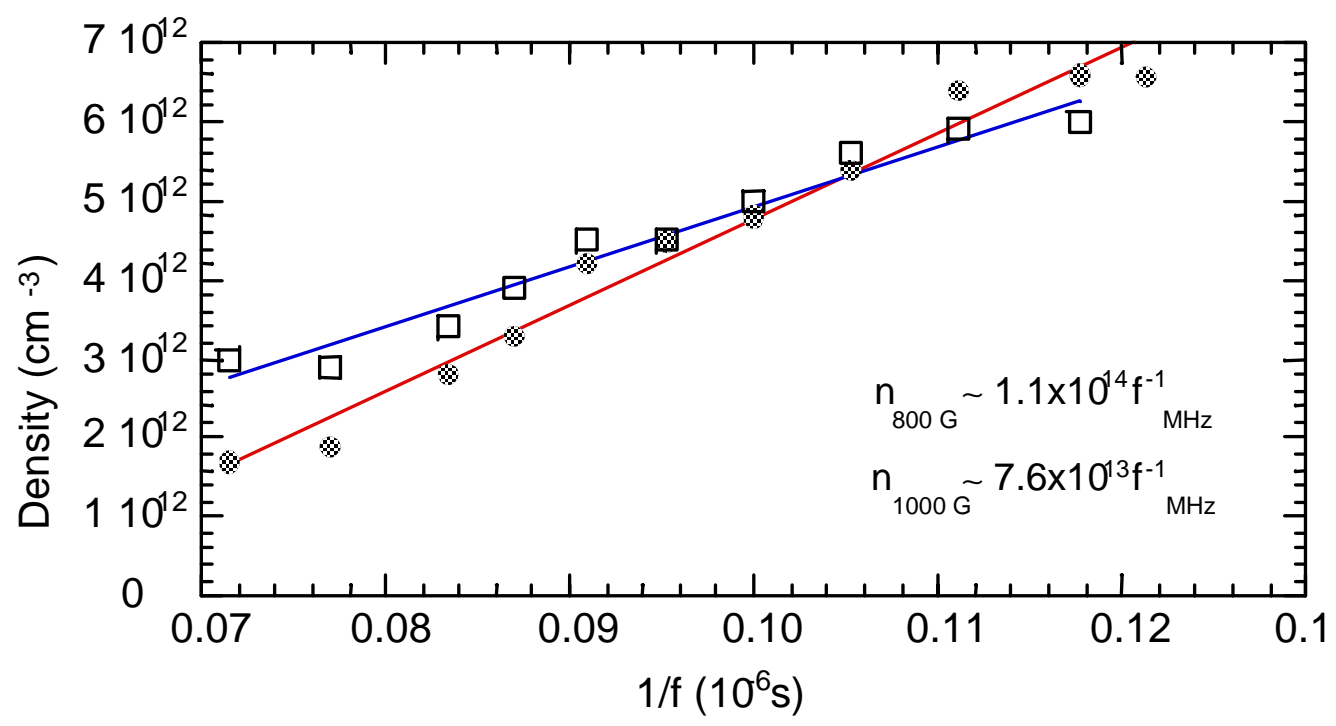

Figure 17: Measurements of the density scaling inverse linearly with driving frequency. ${ }^{37}$ 


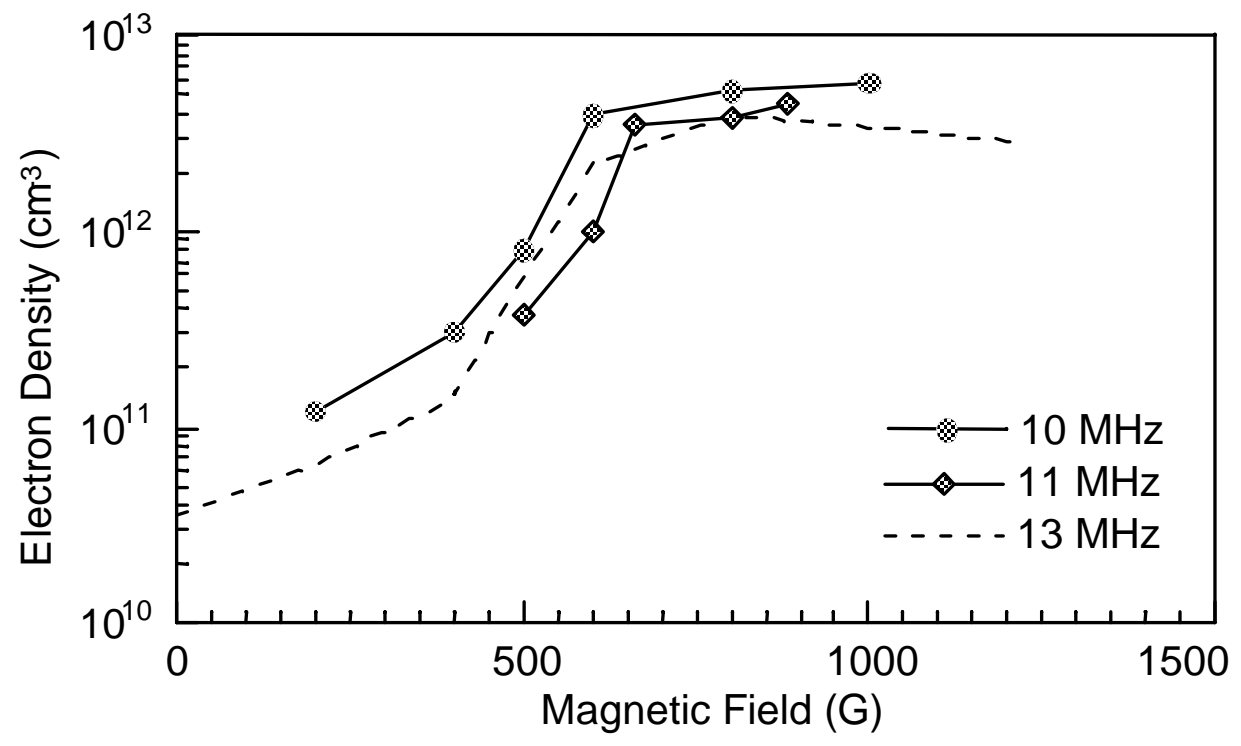

Figure 18: Measurement of electron density versus magnetic field strength for three different driving frequencies in HELIX. The density increases linearly with the magnetic field strength and then levels out.

As pointed out in Keiter et al. ${ }^{37}$ the simple dispersion relation calculation is not applicable to helicon sources where the radius is not much smaller than the length (sources with a moderate aspect ratio). In such cases, the full impact of the boundary conditions must be included in the analysis. A complete derivation of the helicon wave magnetic field components in a cylinder was published by Chen et al., ${ }^{48}$ and only the final equations for the wave magnetic fields are reproduced here. The three magnetic field components are given by:

$$
\begin{gathered}
B_{z}^{\prime \prime}+f(r) B_{z}^{\prime}+g(r) B_{z}=0 \\
k_{\perp}^{2} B_{r}=\frac{i m \alpha}{r} B_{z}+i k_{\|} \gamma B_{z}^{\prime} \\
k_{\perp}^{2} B_{\theta}=-\alpha B_{z}^{\prime}-\frac{m k_{\|} \gamma}{r} B_{z} \\
\text { where } \quad '=\partial / \partial r \text { and } \\
f(r)=\frac{1}{r}-\frac{2 \alpha \alpha^{\prime}}{k_{\perp}^{2}},
\end{gathered}
$$




$$
\begin{gathered}
g(r)=\frac{k_{\perp}^{2}}{\gamma}-\frac{m^{2}}{r^{2}}-\frac{m \alpha^{\prime}}{k_{\|} \gamma r}\left(1+\frac{2 k_{\|}^{2} \gamma^{2}}{k_{\perp}^{2}}\right), \\
\alpha(r)=\frac{\omega \mu_{o} e}{B_{o} k_{\|}} n(r), \\
\gamma=1-\left(\frac{\omega}{c k_{\|}}\right)^{2}, \\
k_{\perp}^{2}=\alpha^{2}-k_{\|}^{2} .
\end{gathered}
$$

Assuming a uniform density profile, $n(r)=n$, and $\omega / k_{\|}<<c$, (neglecting displacement currents) the expressions for $f(r)$ and $g(r)$ can be simplified. Equation (46) becomes

$$
B_{z}^{\prime \prime}+\frac{1}{r} B_{z}^{\prime}+\left(k_{\perp}^{2}-\frac{m^{2}}{r^{2}}\right) B_{z}=0
$$

and equation (53) becomes

$$
k_{\perp}^{2}=\left(\frac{\omega \mu_{o} e}{B_{0} k_{\|}} n\right)-k_{\|}^{2} .
$$

Equation (54) is a standard Bessel function differential equation and the boundary conditions on $B_{z}$ at $r=a\left(B_{r}=0\right)$ due to equation (47) require

$$
0=\frac{m \alpha}{a} J_{m}\left(k_{\perp} a\right)+k_{\|} J_{m}^{\prime}\left(k_{\perp} a\right)
$$

$J_{m}$ and $J_{m}^{\prime}$ are the Bessel function of $m^{\text {th }}$ order and the derivative of the $m^{\text {th }}$ order Bessel function, respectively. For the case of $m=+1$ :

$$
0=\left(\frac{\omega \mu_{0 e}}{a B_{0}} n_{0}\right) J_{1}\left(k_{\perp} a\right)+k_{\|}^{2} J_{1}^{\prime}\left(k_{\perp} a\right)
$$

or, rewriting the equation in terms of only $k_{\perp}$ :

$$
0=\left(\frac{\omega \mu_{0 e}}{a B_{0}} n_{0}\right) J_{1}\left(k_{\perp} a\right)+\left(\frac{-k_{\perp}^{2}+\sqrt{k_{\perp}^{4}+4\left(\frac{\omega \mu_{0} e}{B_{0}} n_{0}\right)^{2}}}{2} J_{1}^{\prime}\left(k_{\perp} a\right) .\right.
$$


Equation (58) can be solved numerically and the $k_{\perp}$ and $k_{\|}$for a given set of plasma parameters compared to measurement. Such high frequency wave field measurements in HELIX are expected in late 1999.

For the case of $m=0$, equation (56) reduces to:

$$
k_{\perp} a=3.83 .
$$

Assuming $k_{\perp} \gg k_{\|}$, and substituting equation (59) into equation (53) yields $\alpha=$ constant. Substituting this into equation (51) recovers the simple dispersion relation of equation (45), assuming a uniform density and a small aspect ratio.

\subsubsection{Possible Explanations of Efficient Ionization in Helicon sources}

Chen was one of the first to suggest Landau damping as a possible ionization mechanism in the helicon source. Linear Landau damping is a process where electrons with velocities approximately equal to the phase velocity of a wave exchange energy with the wave. Electrons with velocities slightly slower than the phase velocity are sped up and those slightly faster than the phase velocity are slowed down (See Figure 19). For the Maxwellian distribution shown in Figure 19, more electrons are traveling slower than the phase velocity than faster. This leads to a net gain in energy for the particles while the wave loses energy and is damped. Chen hypothesized that to maximize the ionization efficiency or density of a small aspect ratio helicon source, the resonant energy of the electrons must be approximately equal to the optimum electron impact ionization energy for the gas. For argon, the optimum electron impact ionization occurs for electron energies of $50-100 \mathrm{eV}$. This led to a period of helicon source design where the antenna length was chosen to generate phase velocities believed to optimally match the electron velocities in such a way to achieve the maximum density for a given driving frequency and magnetic field. Although high-density plasmas were produced in helicon sources designed to maximize Landau damping, ${ }^{49}$ little experimental evidence for Landau damping in helicon sources exists. 


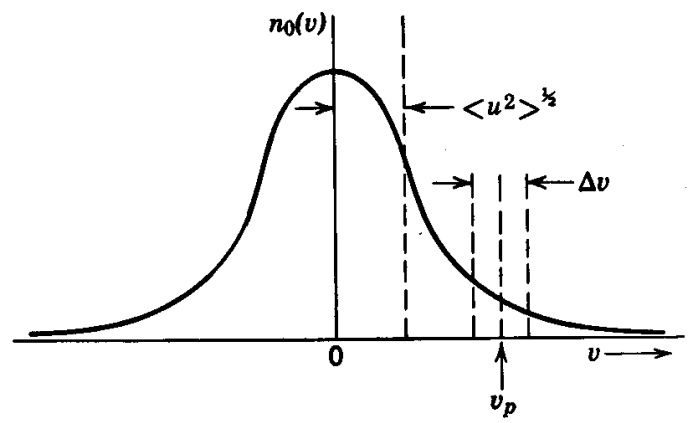

Figure 19: Picture showing the electrons that are involved in the Landau damping process. The wave has a phase velocity of $v_{p}$ and the electrons with velocities in the range of $\Delta v$ are the ones affected. ${ }^{\mathbf{5 0}}$

Helicon groups have looked for evidence of high-energy electrons, greater than $20 \mathrm{eV}$, as one means of substantiating the Landau damping hypothesis. Chen and Decker ${ }^{51}$ observed that one of the endplates of their device charged to large negative potentials even though the temperature of the electrons was only $3 \mathrm{eV}$. They claimed the charging suggested the presence of fast electrons in the source (Figure 20). Early measurements in HELIX also saw charging of the endplate to potentials of less than $-150 \mathrm{~V}$ and indications of fast electrons have also been reported by other groups. ${ }^{34,52}$ However, rf effects can distort Langmuir probe measurements (see Section 3.1.2) and create features that resemble fast electrons in the $\mathrm{I}-\mathrm{V}$ characteristic trace. Thus, the validity of such fast electron measurements is debatable. Calculations of the Landau damping rate predict very little damping in a typical helicon source. For example, using HELIX results from the Nagoya type III antenna and assuming a value of the helicon wavelength of $\lambda \cong 18.8 \mathrm{~cm}$ the Landau damping rate is given by ${ }^{37}$

$$
\begin{gathered}
{\left[\frac{\operatorname{Im}(k)}{\operatorname{Re}(k)}\right]_{L D}=2 \sqrt{\pi} \frac{\mathrm{v}_{t h}}{\omega_{c e}} \frac{3.83}{a}\left(\frac{\omega}{k \mathrm{v}_{t h}}\right)^{4} e^{-\left(\frac{\omega}{k \mathrm{v}_{t h}}\right)^{2}}} \\
{\left[\frac{\operatorname{Im}(k)}{\operatorname{Re}(k)}\right]_{L D} \approx 7 \times 10^{-3}}
\end{gathered}
$$

The collisional damping rate for the same HELIX parameters is: ${ }^{37}$

$$
\left[\frac{\operatorname{Im}(k)}{\operatorname{Re}(k)}\right]_{C D}=\frac{v_{e i}}{\omega} \frac{c^{2}}{\omega_{p e}^{2}}\left(\frac{3.83}{a}\right)^{2}
$$




$$
\left[\frac{\operatorname{Im}(k)}{\operatorname{Re}(k)}\right]_{C D} \approx 2 \times 10^{-3}
$$

Thus, for typical HELIX operating conditions, Landau damping should be larger than collision damping. However, both rates are too small to explain the ionization efficiency, given the input power.

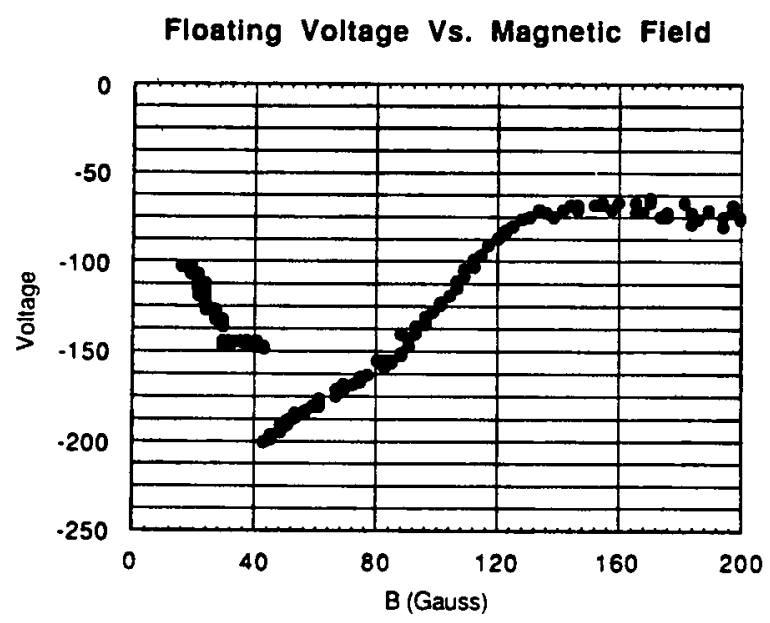

Figure 20: Floating potential versus magnetic field for Chen's experiment. The floating potential of the endplate was as low as $-200 \mathrm{~V}$ for a field of $40 \mathrm{G}$. The magnitude of the floating potential was thought to be caused by fast electrons. ${ }^{\mathbf{5 1}}$

Recently, Chen et al. estimated an upper limit for Landau damping in a helicon source. ${ }^{53}$ Using a gridded energy analyzer and assuming Maxwellian electron distributions, they measured an electron temperature between $3 \mathrm{eV}$ to $3.35 \mathrm{eV}$. For a Maxwellian distribution of such temperatures, the number of fast electrons relative to the bulk can be estimated. Assuming the electrons capable of the most efficient ionization have energies of roughly $50 \mathrm{eV}$, they determined that the $50 \mathrm{eV}$ electrons comprise roughly $0.02 \%$ of the bulk density. Assuming that $\left\langle\sigma v_{i o n}\right\rangle_{50 e V}>400\left\langle\sigma v_{i o n}\right\rangle_{3 e V}$, these fast electrons would still only account for about $10 \%$ of the observed ionization. ${ }^{53}$ Because of the lack of experimental confirmation, Landau damping is no longer considered a likely explanation for the high ionization efficiency of helicon sources.

Another possibility is particle trapping. ${ }^{54}$ Although the process is similar to Landau damping, the resonant electron energy is determined in a somewhat different fashion. By 
multiplying the ionization rate curve by a $3 \mathrm{eV}$ Maxwellian distribution function (Figure 21), Degeling et al. determined that $20 \mathrm{eV}$ electrons yield the maximum ionization rate in an argon plasma with a $3 \mathrm{eV}$ Maxwellian distribution. For appropriate wave frequencies and wavelengths, these $20 \mathrm{eV}$ electrons can be trapped by a large amplitude rf wave field. While trapped, the electrons experience simple harmonic motion due to the potential of the wave and are maintained at their $20 \mathrm{eV}$ kinetic energy. Degeling et al. showed the number of electrons trapped in the rf wave will grow as the electric field amplitude, and hence, as the rf power of the antenna is increased. Although this mechanism seems more likely to result in efficient gas ionization, the available experimental proof is circumstantial.
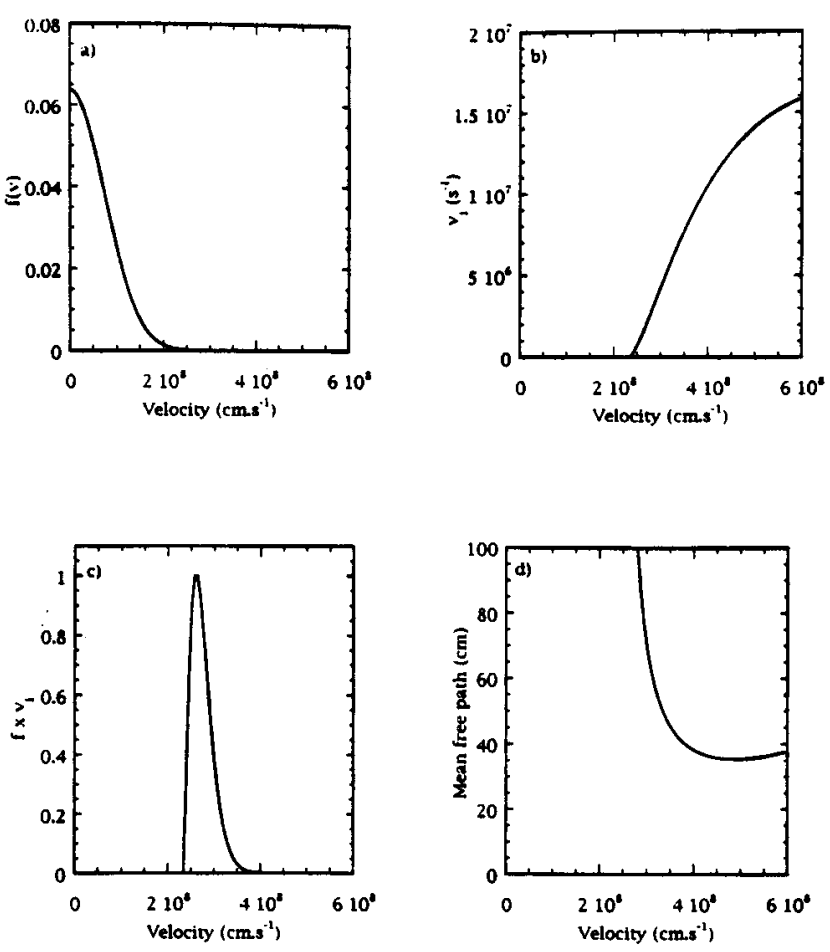

Figure 21: Figures from Degeling et al. ${ }^{54}$ showing a) the distribution function of $3 \mathrm{eV}$ electrons. b) Ionization rate for argon. c) The product of a) and b). The peak velocity corresponds to an energy of roughly $20 \mathrm{eV} \mathrm{d}$ ) The mean free path for ionizing collisions.

While considerable attention has been devoted to looking for evidence of Landau damping or trapped electrons to explain the ionization efficiency, some researchers have begun to investigate the observed wave characteristics by examining the coupling 
between helicon waves (modes) and Trivelpiece-Gould (TG) waves (modes). Experiments have shown that at very low fields $(<100 \mathrm{G})$, density peaks occur at specific field strengths. ${ }^{55,51,56}$ Chen originally attributed these density enhancements to electron cyclotron resonances. ${ }^{56}$ Although electron inertia had been considered much earlier, ${ }^{57,58}$ the possible role of Trivelpiece-Gould (TG) electrostatic modes, which arise from electron inertia effects, was not considered. Shamrai and Taranov first discussed TG modes and introduced the terminology of resonances and anti-resonances in a helicon plasma. ${ }^{59}$ A resonance occurs when both the helicon wave and the TG wave can exist in the plasma. An anti-resonance is when either the helicon or the TG wave is not able to exist in the plasma. In their derivation, they assumed the device has a low aspect ratio and the wave frequency is greater than the lower hybrid frequency. The TG mode is an electron-cyclotron wave in a cylinder and has a shorter radial wavelength than the helicon mode. The TG mode damps with increasing magnetic field, but at low fields, the two waves have similar mode structure. Thus, the observed density peaks at low $B$ fields may result from improved coupling to TG modes at particular field strengths. ${ }^{60}$ At the lower hybrid frequency, the slow wave changes from an electron wave to an ion wave and only the helicon mode exists. ${ }^{60}$ This wave transition might also be responsible for the density peaks observed at the lower hybrid frequency in higher magnetic field experiments. ${ }^{38,46}$ For high plasma density and high magnetic field, the waves will be predominantly electromagnetic and the helicon component will dominate. For low magnetic fields or driving frequencies near the cyclotron frequency, electron inertia will dominate, causing the electrostatic TG wave to dominate.

At this time, there is no conclusive experimental evidence that TG modes propagate in low field helicon sources. Since the TG modes should dominate when the driving frequency is near the electron cyclotron frequency and few experiments run at these parameters, experimental identification of TG modes in low field helicon sources may not be available for some time. 


\subsection{Space Simulation Chamber}

The space simulation chamber, LEIA is large aluminum cylindrical chamber on loan from Massachusetts Institute of Technology (MIT). LEIA has an inner diameter of $1.8 \mathrm{~m}$ and a length of $4.4 \mathrm{~m}$. There are seven $2.5 \mathrm{~m}$ diameter magnet coils spaced along the length of LEIA to produce a uniform magnetic field. Each magnet contains 20 turns of 0.36" x 0.41" hollow rectangular aluminum tubing. Using a 200 Amp DC EMHP power supply, the magnetic field can be varied from 0 to 70 Gauss. The magnetic field in LEIA is given by:

$$
B=-1.09+.33 I_{L}+.022 I_{H}
$$

where $I_{L}$ is the current in the LEIA coils and $I_{H}$ is the current in the HELIX coils. Figure 22 and Figure 23 show the radial and azimuthal magnetic field profiles in LEIA. The magnetic field in HELIX significantly influences the magnetic field strength in LEIA near the bellows joining the two devices $(z>300 \mathrm{~cm})$.

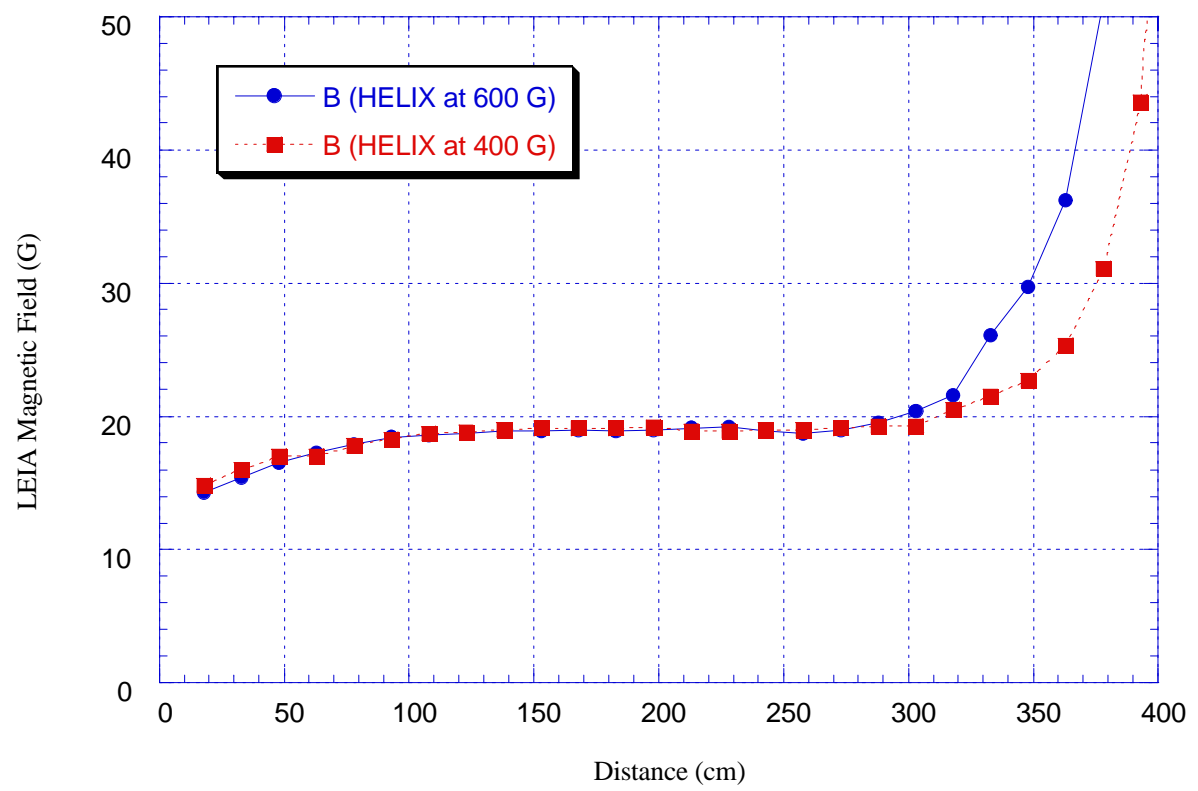

Figure 22: Azimuthal magnetic field profile in LEIA. Note that as the field in HELIX is increased, the magnetic profile becomes steeper for $z>300 \mathrm{~cm}$. 


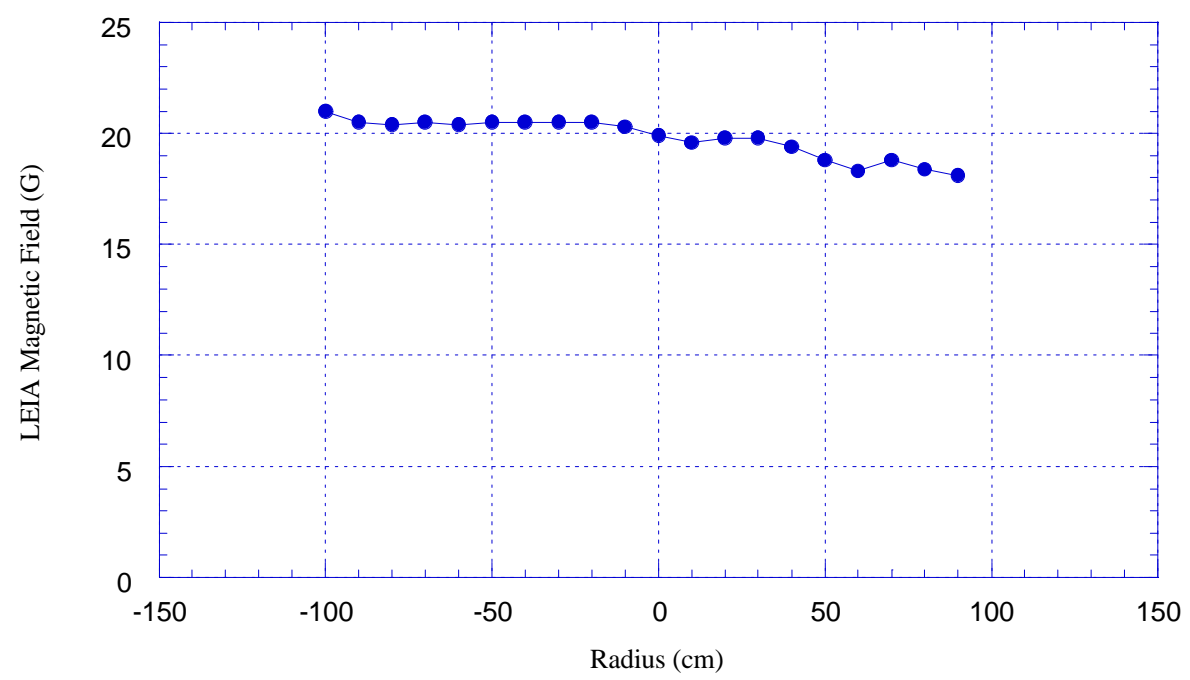

Figure 23: Radial Profile of magnetic field in LEIA.

At the far end of LEIA are two Balzers TMU 1600 turbomolecular pumps. A nearby pressure gauge is used to measure the neutral pressure in LEIA. The differential pumping design creates a neutral pressure gradient in LEIA. Thus, the neutral pressure in LEIA is typically five to ten times lower than in HELIX.

Typical operating parameters for LEIA are shown in Table 4. The magnetic field strengths in HELIX and LEIA, the pressure and the rf power can be manipulated to control the plasma density and ion temperature in LEIA. The mechanism responsible for the large ion temperatures observed in LEIA is currently unknown but is under investigation. 
Table 4: Typical operating parameters of LEIA.

\begin{tabular}{|c|c|}
\hline Parameter & Typical LEIA Values \\
\hline LEIA Length & $4.4 \mathrm{~m}$ \\
\hline LEIA Radius & $1.8 \mathrm{~m}$ \\
\hline Base Pressure & $<5 \times 10^{-7} \mathrm{Torr}$ \\
\hline Operating Pressure & $.1-5 \mathrm{mTorr}$ \\
\hline Magnetic Field & $<70 \mathrm{G}$ \\
\hline Density & $<3 \times 10^{13} \mathrm{~cm}^{-3}$ \\
\hline Electron Temperature & $<10 \mathrm{eV}$ \\
\hline Ion Temperature & $1-20$ \\
\hline Anisotropy $\left(T_{i \perp} / T_{i \|}\right)$ & $<.7 \mathrm{~mm}($ for $5 \mathrm{eV})$ \\
\hline Electron Gyroradius & $<1.6 \mathrm{~mm}($ for $0.5 \mathrm{eV})$ \\
\hline Ion Gyroradius & $<0.08$ \\
\hline Ion Beta & $<1.15$ \\
\hline Electron Beta & $1-140$ \\
\hline Collisionallity $\left(v_{i i} / \omega_{c i}\right)$ & \\
\hline
\end{tabular}

\subsection{Data Acquisition System}

The data acquisition system consists of a Tektronix 16 channel VX4780 signal conditioner, a $200 \mathrm{kHz}$ Tektronix VX4244 16 channel, 16 bit digitizer, and a $133 \mathrm{MHz}$ Pentium PC computer. The signal conditioner is used for frequency filtering and differential measurements of signals before the signals are recorded by the digitizer. Differential measurements from 9 different magnetic sense coils, a Langmuir probe, and two different laser induced fluorescence (LIF) probes can all be recorded simultaneously. The data acquisition system is controlled through projects written in LabWindows/CVI software. The $200 \mathrm{kHz}$ digitation rate limits the frequencies of the magnetic 
measurements to $100 \mathrm{kHz}$. For magnetic fluctuation measurements, 2048 points are recorded, giving a frequency resolution of $97 \mathrm{~Hz} .1000$ points are recorded for the Langmuir probe and LIF measurements. 


\section{Diagnostics}

The LEIA diagnostics used for these experiments included a Langmuir probe, two LIF probes separated axially, and nine magnetic sense coils separated axially, radially, and azimuthally. The nine magnetic sense coils can determine the wavelengths of electromagnetic instabilities in LEIA in the $\hat{r}, \hat{\theta}$, and $\hat{z}$ directions. The LIF, magnetic, and Langmuir probes were mounted on linear motion Velmex stages, capable of submillimeter position resolution. The probes perturbed the plasma somewhat, but only seemed to affect downstream measurements on the same field line. The upstream measurements were not affected by the downstream probes. In HELIX, the only diagnostic used was LIF.

\subsection{Langmuir Probe}

A Langmuir probe is essentially a conductor inserted into a plasma. The conductor is then biased with a voltage and the current to the probe measured. ${ }^{61}$ The relationship between the biasing voltage and the collected current is referred to as an I-V characteristic, or an I-V trace. A typical I-V trace is shown in Figure 24. 


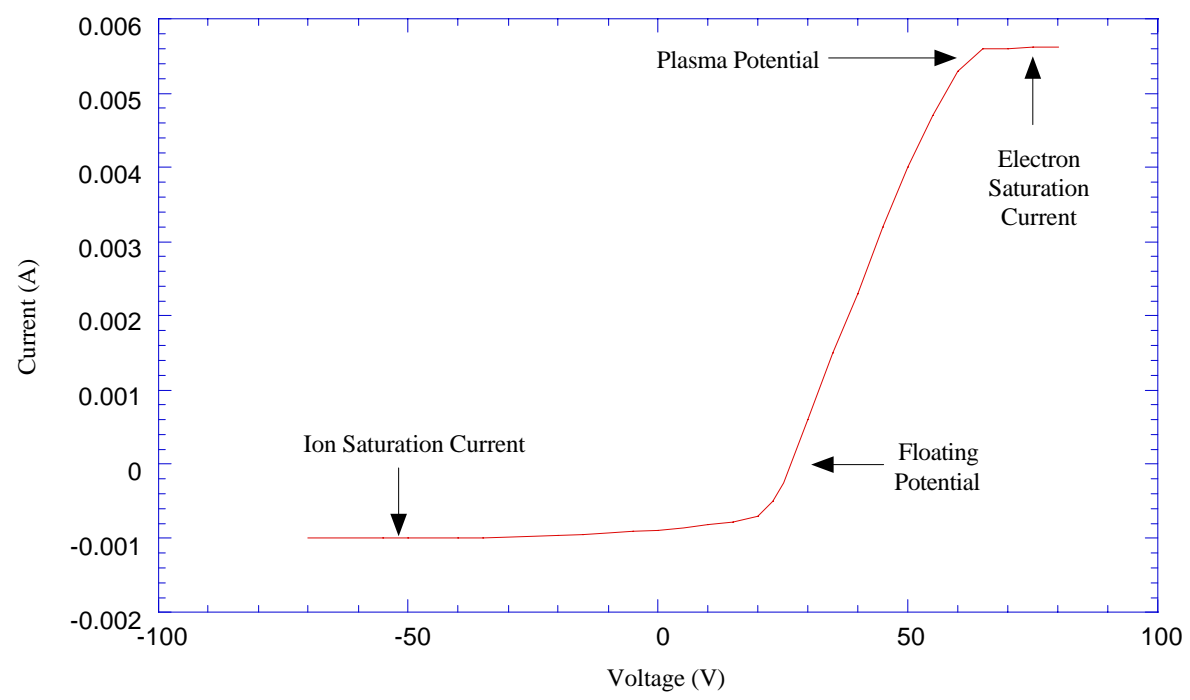

Figure 24: Hypothetical Langmuir probe trace.

When a Langmuir probe is placed into a plasma, the probe typically becomes negatively charged. This is because the electrons are usually more mobile in the plasma than the positive ions and thus the electron flux to the probe is greater than the ion flux. The voltage that an unbiased probe charges to is called the floating potential. As the probe is biased more negative than the floating potential, the probe collects ion current. Eventually, a limit is reached when the probe is collecting as much ion current as it can possibly collect given the plasma density and temperature. This is known as the ion saturation current. If the probe is biased more positive than the floating potential, electrons are collected. As seen in Figure 24, right after the floating potential the I-V trace takes a sharp turn upward. The bend is also referred to as the "knee". As the positive bias is increased on the probe, eventually the collected electron current reaches a plateau. This is referred to as the electron saturation current. 


\subsubsection{Determination of electron density and temperature}

Assuming the plasma is collisionless and there is no magnetic field, the current in the region around the knee can be approximated by: ${ }^{61}$

$$
I\left(V_{o}\right)=n_{\infty} e A_{p}\left(\frac{T_{e}}{m_{i}}\right)^{1 / 2}\left[\frac{1}{2}\left(\frac{2 m_{i}}{\pi m_{e}}\right)^{1 / 2} \exp \left(\frac{e\left(V_{o}-V_{p}\right)}{T_{e}}\right)-\frac{A_{s}}{A_{p}} \exp \left(-\frac{1}{2}\right)\right]
$$

where $m_{e}$ is the electron mass, $m_{i}$ is the ion mass $A_{s}$ is the area of the sheath, $A_{p}$ is the surface area of the probe, $V_{p}$ is the plasma potential and $V_{o}$ is the applied voltage. The sheath around a Langmuir probe is a region of space a few Debye lengths thick. The sheath is a region of spatially varying potential and is created when the ions in the plasma Debye shield the potential applied to the probe. ${ }^{62}$ The two unknowns in equation (65) are $n_{\infty}$, the electron density far from the probe, and $T_{e}$, the electron temperature. The derivative of the current with respect to the bias voltage is:

$$
\frac{d I}{d V_{o}}=\frac{e}{T_{e}}\left(I-I_{s i}\right)+\frac{d I_{s i}}{d\left(V_{o}-V_{p}\right)}
$$

where $I_{s i}=-e J_{i}$ and

$$
J_{i}=n_{\infty} A_{p}\left(T_{e} / m_{i}\right)^{1 / 2}
$$

Since the ion saturation current, $I_{s i}$, is relatively constant, $d I / d V_{o}>>d I_{s i} / d V_{o}$.

Therefore, $T_{e}$ can be approximated by

$$
T_{e}=e\left(I-I_{s i}\right) / \frac{d I}{d\left(V_{o}-V_{p}\right)} .
$$

Once the electron temperature is obtained from the slope of the I-V characteristic, it is straightforward to use equation (67) to calculate the electron density of the plasma from the measured ion saturation current. As the $V_{p}$ was not determined, $V_{f}$ was used.

Langmuir probes are often used in plasmas with a strong magnetic field. The charged particles no longer move in straight lines but in gyro-orbits. Motion across field lines is restricted while motion along the field lines is basically the same as without a magnetic field. The electrons are more strongly affected than the ions because their gyro-orbits are 
much smaller than that of the ions (assuming the electrons and ions are approximately the same temperature). Thus, the electron saturation current decreases with a stronger magnetic field. ${ }^{61}$ For cylindrical probes, the importance of magnetic field effects scales with the ratio of the gyro-radius to the probe radius. When this ratio is much less than one for a particle species, then that species is impeded from reaching the probe and the equations (65) through (68) must be modified by including collisions and estimates of cross field transport. ${ }^{61}$ For LEIA, assuming a typical field (35 G), an ion temperature of $0.3 \mathrm{eV}$, and electron temperature of $5 \mathrm{eV}$, the ion gyro-radius is roughly $2.5 \mathrm{~mm}$ and the electron gyro-radius is about $1.5 \mathrm{~mm}$. Since the Langmuir probes used have radii of 0.5 $\mathrm{mm}$, both species have a gyroradius to probe radius ratio larger than 1 . Therefore, the field free formulae should provide a good approximation for the electron temperature and the density in LEIA.

\subsubsection{Langmuir Probe Design}

Plasmas created by a sinusoidal, time-varying signal can influence the I-V trace of a Langmuir probe by distorting the electron retardation region and also shifting the floating potential more negative. ${ }^{63}$ For Maxwellian electron distributions, an accurate electron temperature can be obtained from the average electron current as long as the electron current stays in the exponential region of the $\mathrm{I}-\mathrm{V}$ characteristic, i.e., near the knee. However, if the rf potential forces the electron current outside of the exponential region, then the electron temperature can be overestimated by traditional analysis methods. Besides electron temperature overestimates, the I-V characteristic can also be distorted by the rf to resemble the I-V characteristic of a Maxwellian distribution with a hot tail. ${ }^{63,64}$ To compensate for this, Sudit and Chen developed a method of probe construction to eliminate the rf influence on the Langmuir probe. ${ }^{65}$ Their method is similar to a method developed by Godyak et al.. ${ }^{66}$ 


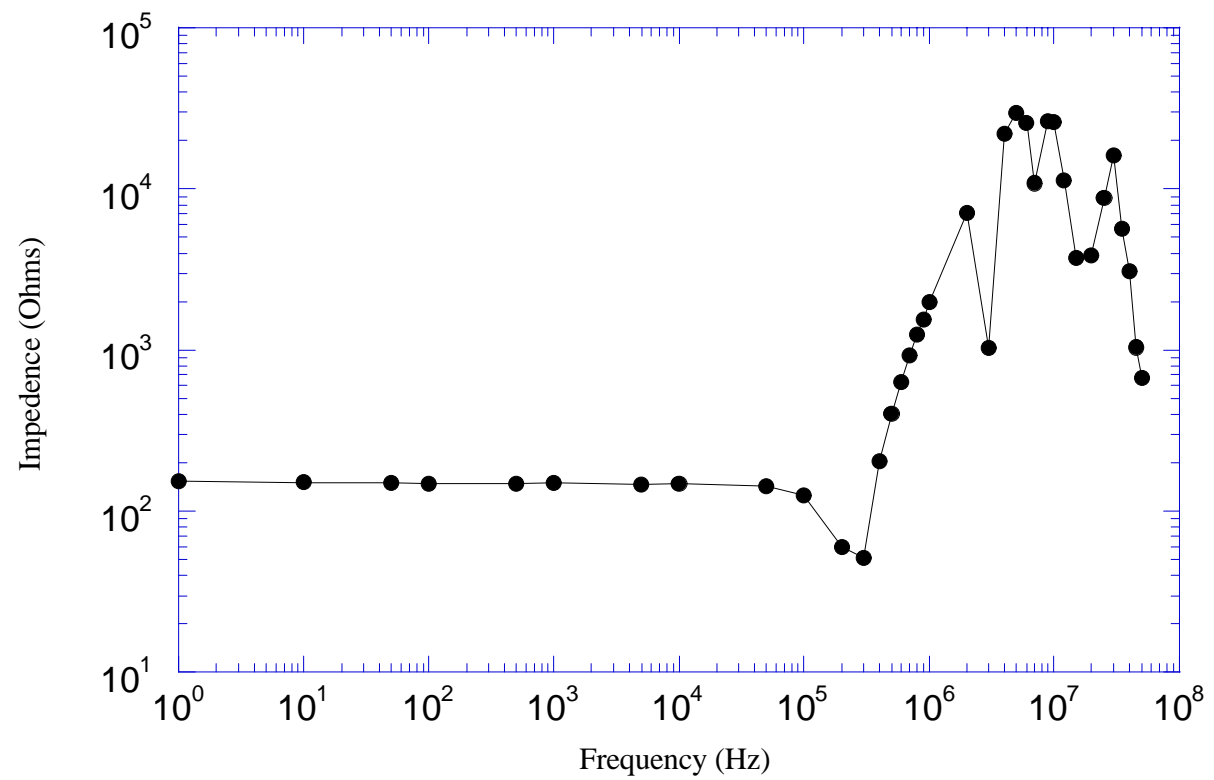

Figure 25: Typical frequency response of the Langmuir probe used in LEIA.

Physically, there are two modifications made to a standard Langmuir probe. The first is the addition of a floating electrode. ${ }^{65}$ The electrode is exposed to the plasma potential fluctuations and connected to the Langmuir probe tip via a large capacitor. This helps to lower the sheath impedance and forces the Langmuir probe tip follow the plasma potential oscillations; thereby reducing the distortion in the trace. The second modification is a chain of $\mathrm{rf}$ chokes. These are placed after the probe tip, but before the current is measured. The chokes increase the impedance of the circuit at the rf frequency. The impedance as a function of frequency for a typical Langmuir probe used in LEIA is shown in Figure 25.

In LEIA, a Keithley 2400 SourceMeter is used to measure the I-V characteristic of the Langmuir probe from $-50 \mathrm{~V}$ to $+50 \mathrm{~V}$. The SourceMeter is controlled through a GPIB interface installed in the PC. A schematic drawing of the Langmuir probe used in LEIA is shown in Figure 26. The probe is mounted on a motorized Velmex drive that allows the radial profile of the electron temperature and density to be measured at a particular $z$ position. To measure the plasma density at different axial positions, the probe is 
physically moved to another port. A typical Langmuir probe trace from LEIA is shown in Figure 28.

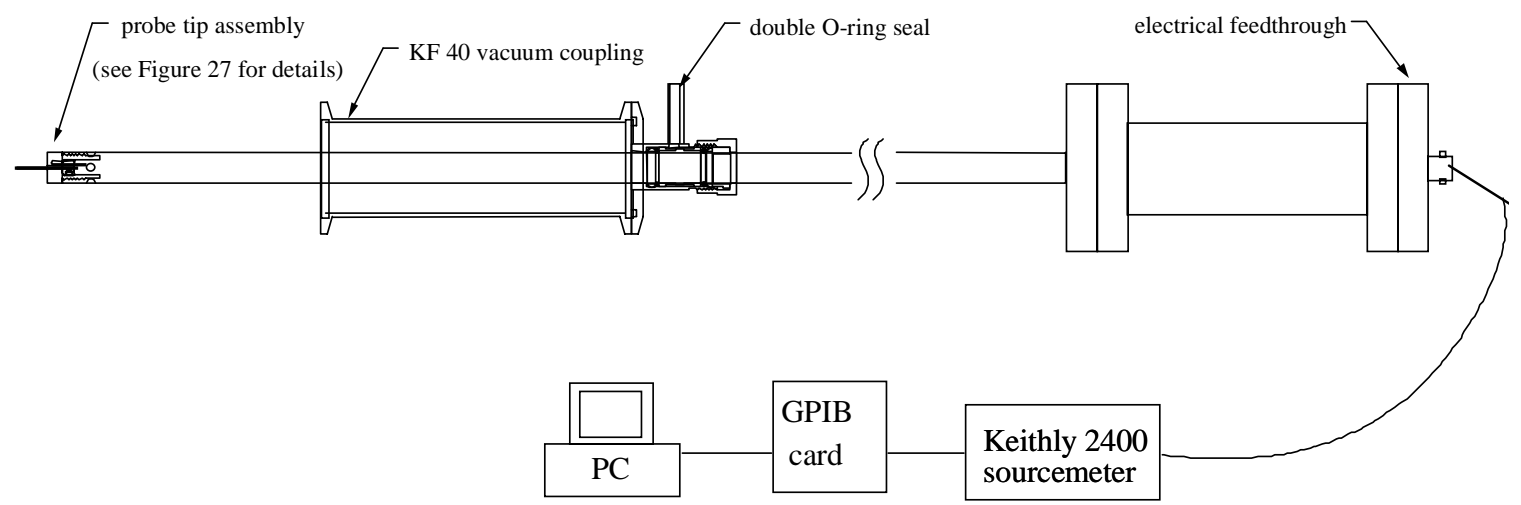

Figure 26: Schematic drawing of the Langmuir probe and measurement circuit.

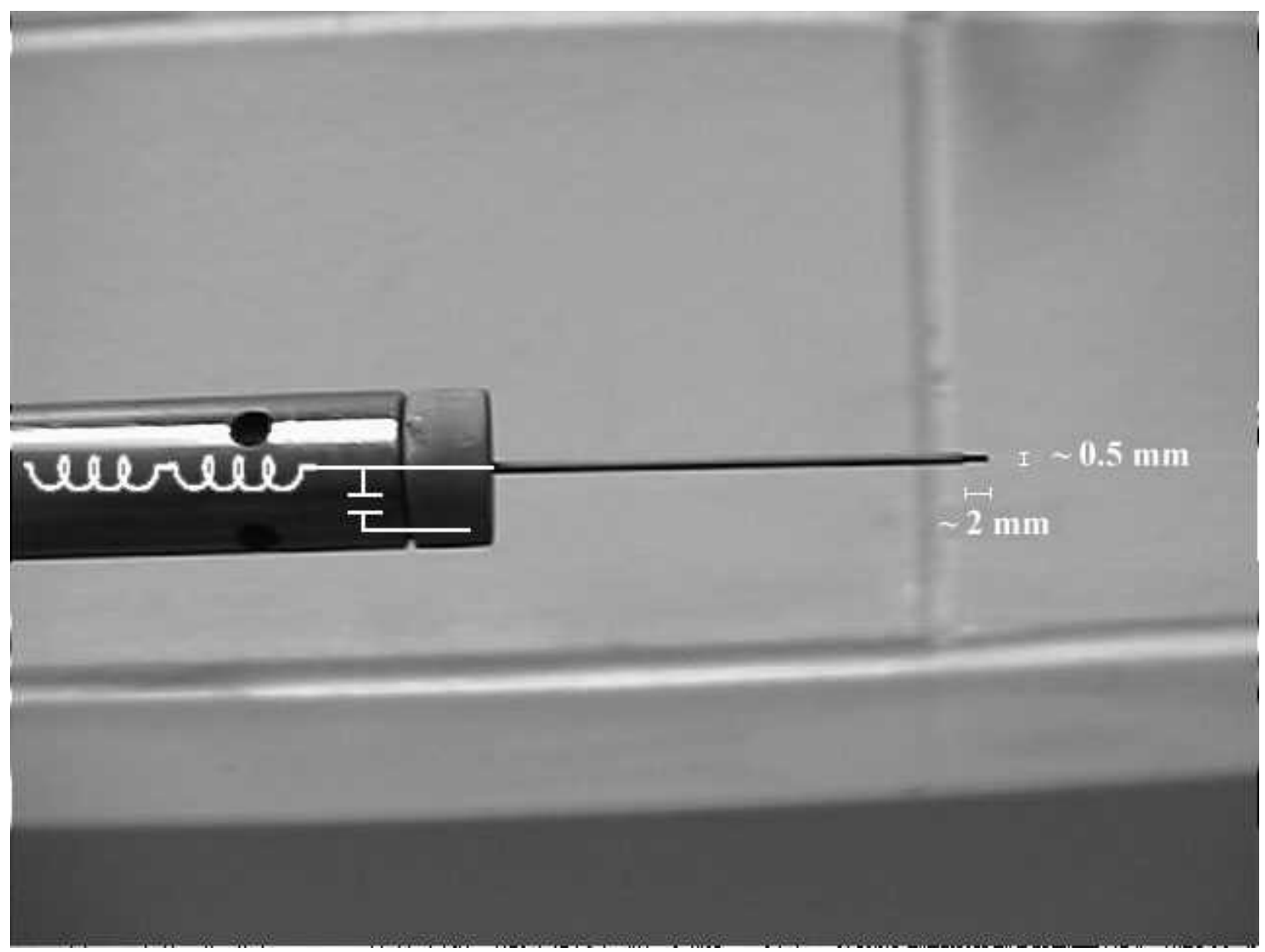

Figure 27: Photograph of the Langmuir probe head. The exposed graphite tip is $2 \mathrm{~mm}$ long and runs the length of the alumina tube into the boron-nitride cap. The capacitor 
between the graphite tip and a small copper rod has a value of approximately $5 \mathrm{nF}$. Five inductors having values between 15 and $270 \mu \mathrm{H}$ are placed in series behind the capacitor.

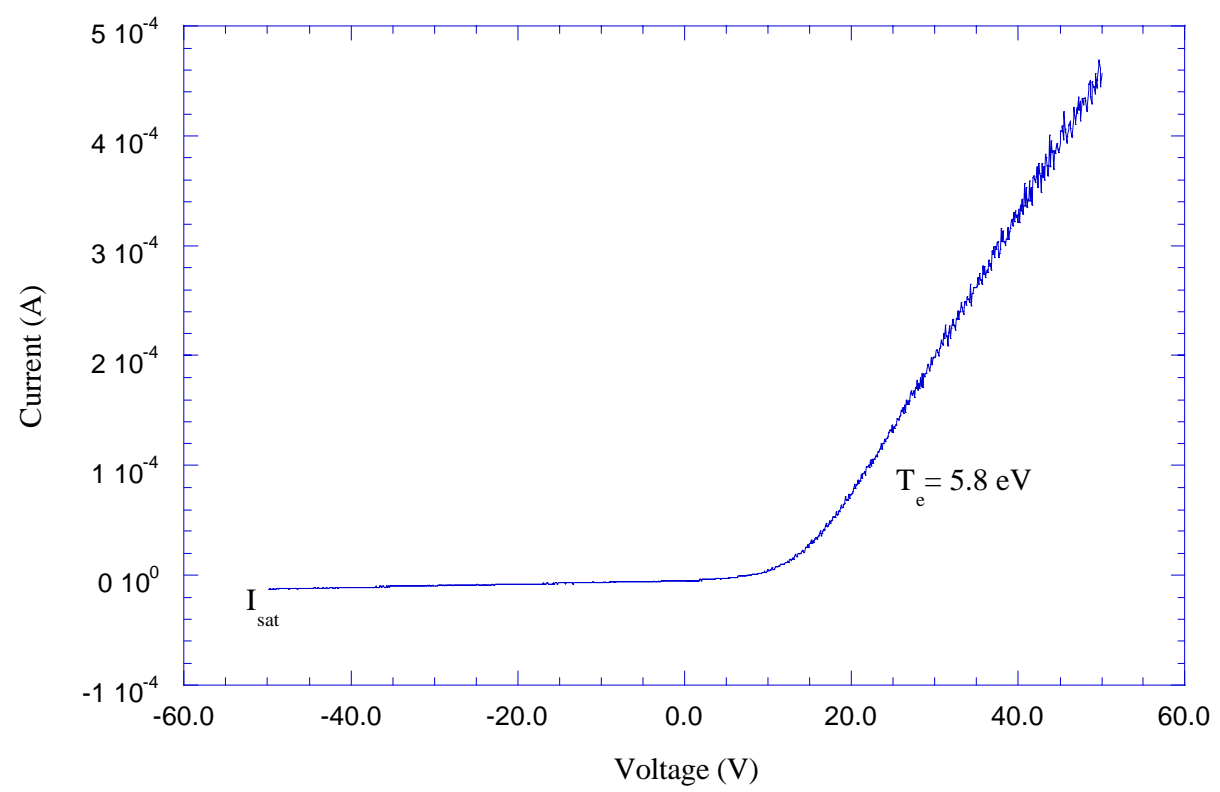

Figure 28: Typical Langmuir probe trace in LEIA.

Electron temperatures in LEIA are typically $5-7 \mathrm{eV}$. These values are somewhat higher than the 3-5 eV temperatures reported by other helicon groups. The likely culprit is differences in the rf compensation of the Langmuir probes. The chokes used in the LEIA probes are not optimized for the $8-10 \mathrm{MHz}$ rf frequencies used in HELIX. In addition, there might be some electron heating as the plasma expands from HELIX into LEIA and the plasma density drops. 


\subsection{Laser Induced Fluorescence}

One of the key diagnostics used in these experiments is laser induced fluorescence (LIF). LIF was first suggested as a plasma diagnostic in 1968 by Measures. ${ }^{67}$ LIF is based upon the selective excitation of an atomic transition by the absorption of laser radiation of the appropriate wavelength. LIF can be used to observe the following:

(i) Ion and atomic velocity (zeroth order) distribution functions (hence ion and atomic temperatures) and densities;

(ii) Ion and atomic particle trajectories;

(iii) Electron temperature from the relative populations of the excited levels;

(iv) The vector direction and magnitude of the local magnetic field by means of the Zeeman effect;

(v) The effective ion charge and the local electric field by means of the Stark effect;

(vi) Ion and atomic first order distribution functions. This can yield the value of the perturbing potential and lead to wavelength measurements of electrostatic waves. $^{68,69}$

In this work, only techniques for (i) will be discussed in detail. A discussion of technique (ii) can be found in the work of McChesney. ${ }^{70}$ Discussion of techniques (iv) and (v) can be found in the work of Moore et al. ${ }^{71}$ and West et al., ${ }^{72}$ respectively. Technique (iii) can only be applied to high-density plasmas, where the ions are in local thermodynamic equilibrium.

The absorption and emission frequencies of an atom or ion moving relative to a radiation source are Doppler shifted by an amount proportional to the component of velocity along the radiation direction. During a typical LIF measurement, the frequency of a narrow bandwidth laser is swept across a collection of ions or atoms that have a thermally broadened velocity distribution. The absorption spectrum for the entire ensemble of atoms or ions has associated with it a width and a shift from the natural frequency. The width is used to determine the temperature and the shift to determine flow velocity of the particle distribution. The LIF system used in these experiments consists of 
a 6 W Coherent Innova 300 argon-ion laser that pumps a Coherent 899 ring dye laser (Figure 29). A beam splitter diverts $10 \%$ of the dye laser's light into a Burleigh 1500 wavemeter to monitor the wavelength. The wavemeter has a resolution of $0.0001 \mathrm{~nm}$. For argon plasmas, the output of the dye laser is tuned to $611.5 \mathrm{~nm}$ to match the $3 \mathrm{~d}^{2} \mathrm{G}_{9 / 2}$ to $4 \mathrm{p}^{2} \mathrm{~F}_{7 / 2}$ transition of singly ionized argon ions. As the dye laser performs a $10 \mathrm{GHz}$ sweep, corresponding to a wavelength sweep of approximately $0.012 \mathrm{~nm}$, the fluorescent emission $(461.1 \mathrm{~nm})$ from the upper metastable level (Figure 30) is measured with a filtered photomultiplier tube detector.

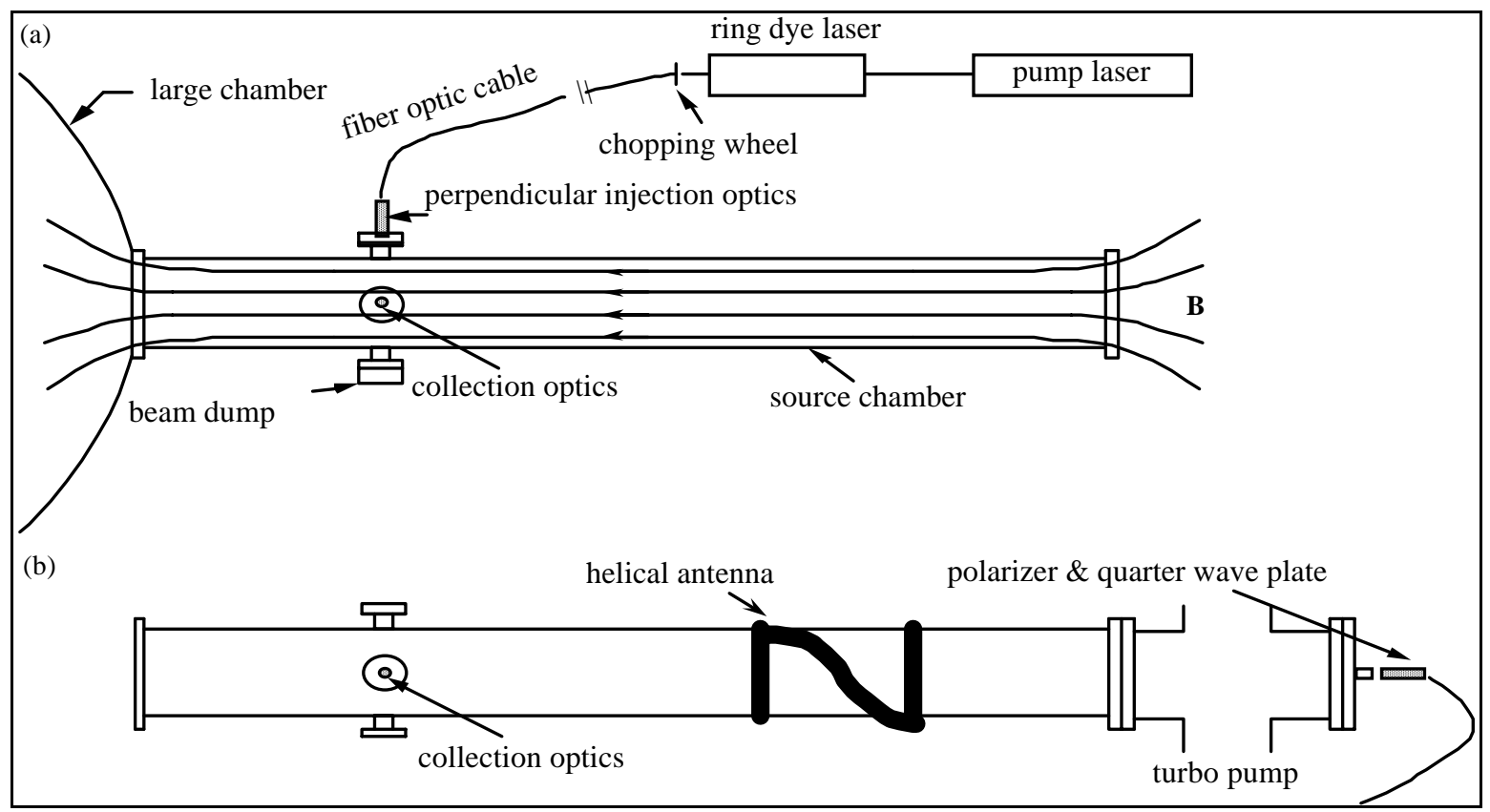

Figure 29: Schematic of LIF system in HELIX. a) Schematic of the perpendicular injection LIF scheme. b) The parallel LIF injection scheme.

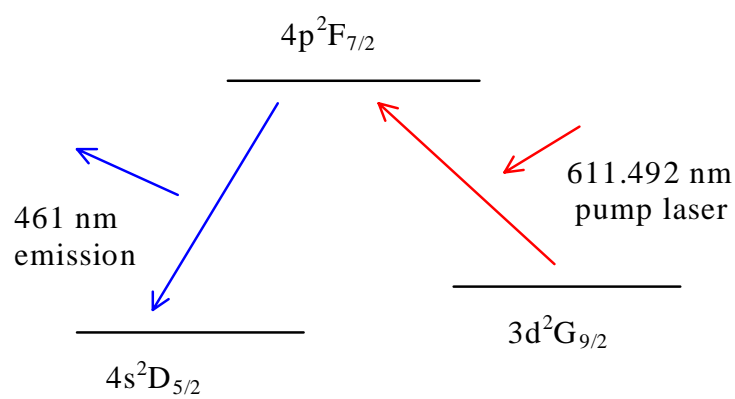

Figure 30: Argon LIF scheme used for ion temperature measurements. 
The filter in front of the photomultiplier has a $1.0 \mathrm{~nm}$ passband centered around the emission line. The output of the dye laser is chopped at $1 \mathrm{kHz}$. The chopping signal is used as the reference for a Stanford Research SR830 lock-in amplifier that monitors the photomultiplier tube signal and distinguishes the fluorescence signal from the intense background emission at the same wavelength using phase synchronous detection. The amplified signal from the SR830 and a measurement of the laser power during the sweep are sent to the Tektronix VXI 4244 digitizer. The LIF signal is normalized by the laser power measurement and is then fitted to a single Gaussian distribution to determine the ion temperature and the center frequency of the ion distribution.

In HELIX, perpendicular and parallel components of the argon ion temperature are obtained by injecting the laser light into the plasma perpendicular and parallel to the magnetic field, respectively. In both cases, the emitted light is collected perpendicularly to the magnetic field (Figure 29). For perpendicular measurements, only the linearly polarized $\pi$ transitions are excited. For the parallel measurements, two circularly polarized $\sigma$ transitions are excited. Because the laser light is transported through a multimode fiber, the polarization of the laser is not preserved. Since the Zeeman splitting of the $\sigma$ lines is on the order of the thermal broadening in HELIX, Zeeman splitting for parallel measurements cannot be ignored. By introducing a combination of a linear polarizer followed by a quarter wave plate into the parallel injection optics, a single circular polarization is selected and only one of the two $\sigma$ transitions is excited. The additional optical components reduce the overall intensity of the laser light and lower the signal-to-noise ratio for parallel ion temperature measurements.

In LEIA, LIF is accomplished with two different LIF probes. One is a re-entrant probe that contains miniature collection optics in a glass tube inserted into the plasma (Figure 31a). The laser is injected from an external port and aligned with the collection optics (Figure 31b). The other is a fully in-situ probe (Figure 32). The in-situ design allows the injection and collection optics to be placed much closer to each other $(\sim 5 \mathrm{~cm})$ than if external ports on the machine were used, thereby increasing the signal to noise ratio. Another advantage of the in-situ LIF probe is that it can be used to measure radial profiles of the ion temperature and perform tomographic surveys of the plasma. Since the 
magnetic field in LEIA is on the order of $50 \mathrm{G}$, Zeeman splitting of the argon lines in LEIA is not a concern.

A)

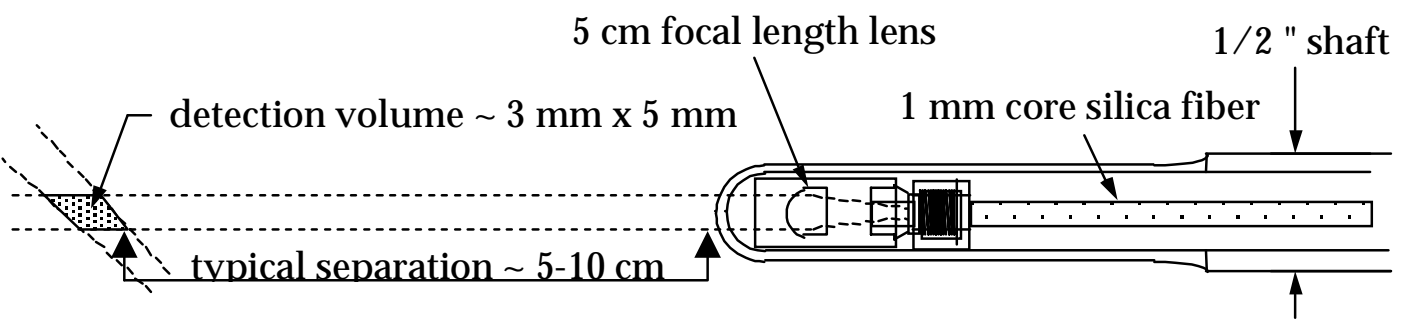

B)

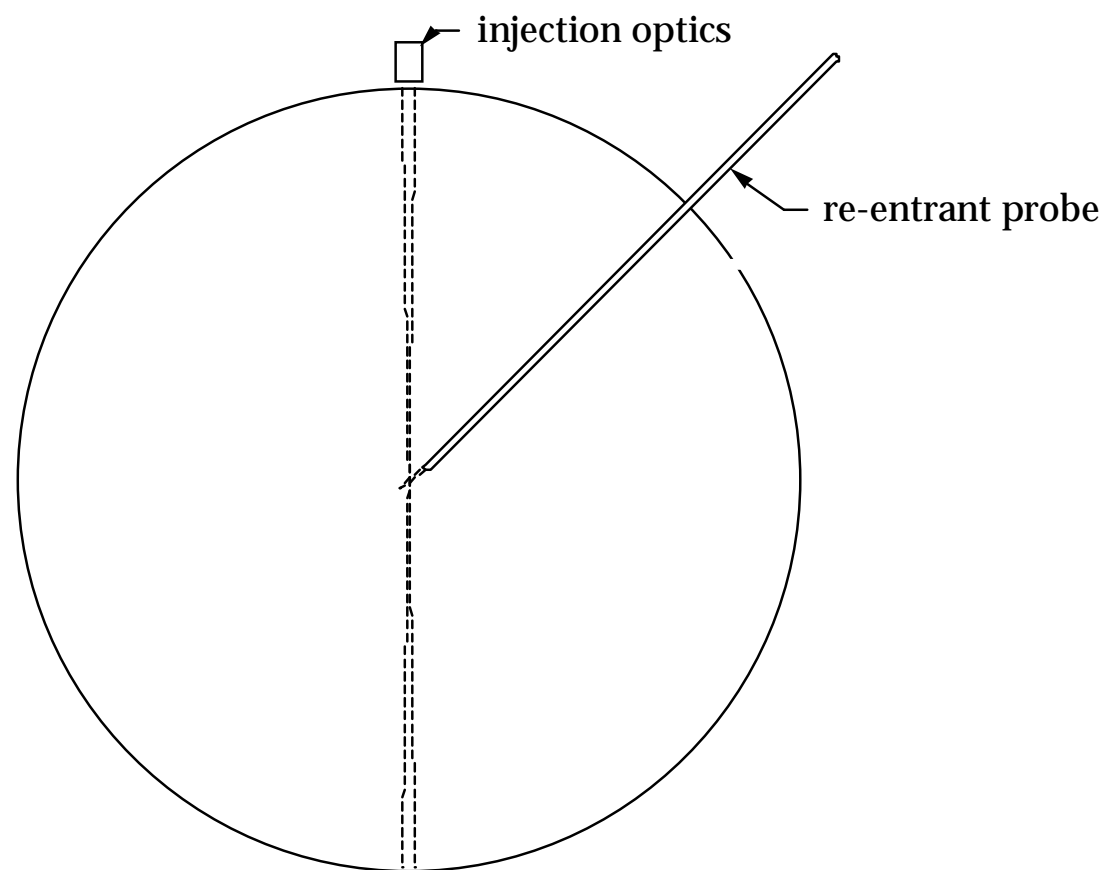

Figure 31: a) Schematic of re-entrant LIF probe. Emission from the $461.1 \mathrm{~nm}$ line is focused into the fiber with a 1/4" plano-convex lens. The fiber is connected to the same filtered PMT that is used for the HELIX measurements. b) Configuration of re-entrant probe inside of LEIA for $T_{\perp}$ measurement. The laser light is injected from the top of LEIA, and focused at the center of the plasma. The re-entrant probe collects the light emitted by the ions in the region defined by the intersection of the collection and injection focal paths.

The two LIF probes are located approximately $65 \mathrm{~cm}$ apart, thereby allowing the ion temperature to be studied at two axial positions in the plasma. The re-entrant probe is 
placed closer to the plasma source and is referred to as the upstream probe. The in-situ probe is referred to as the downstream probe.

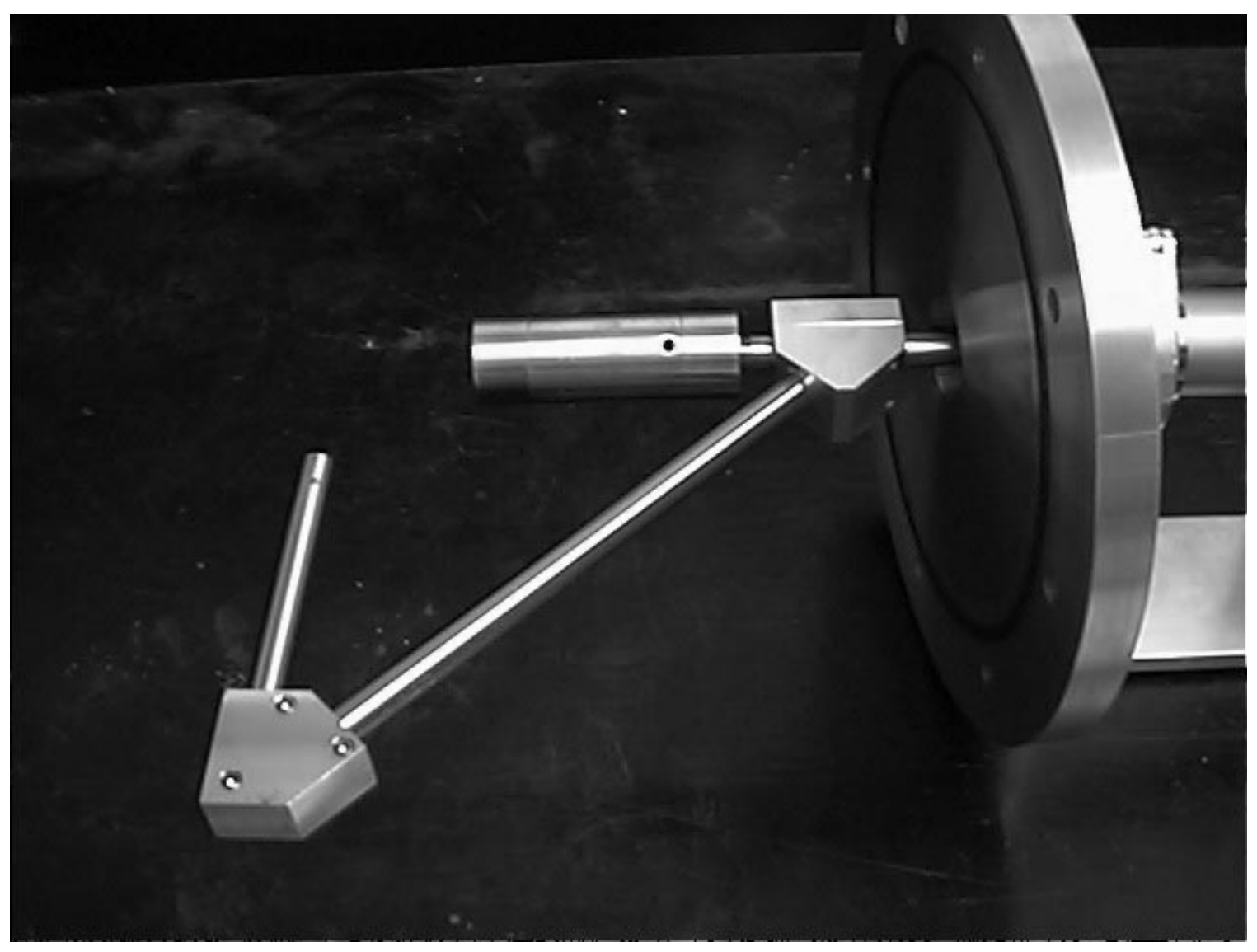

Figure 32: Picture of the head of the radially scanning, in-situ LIF probe. 


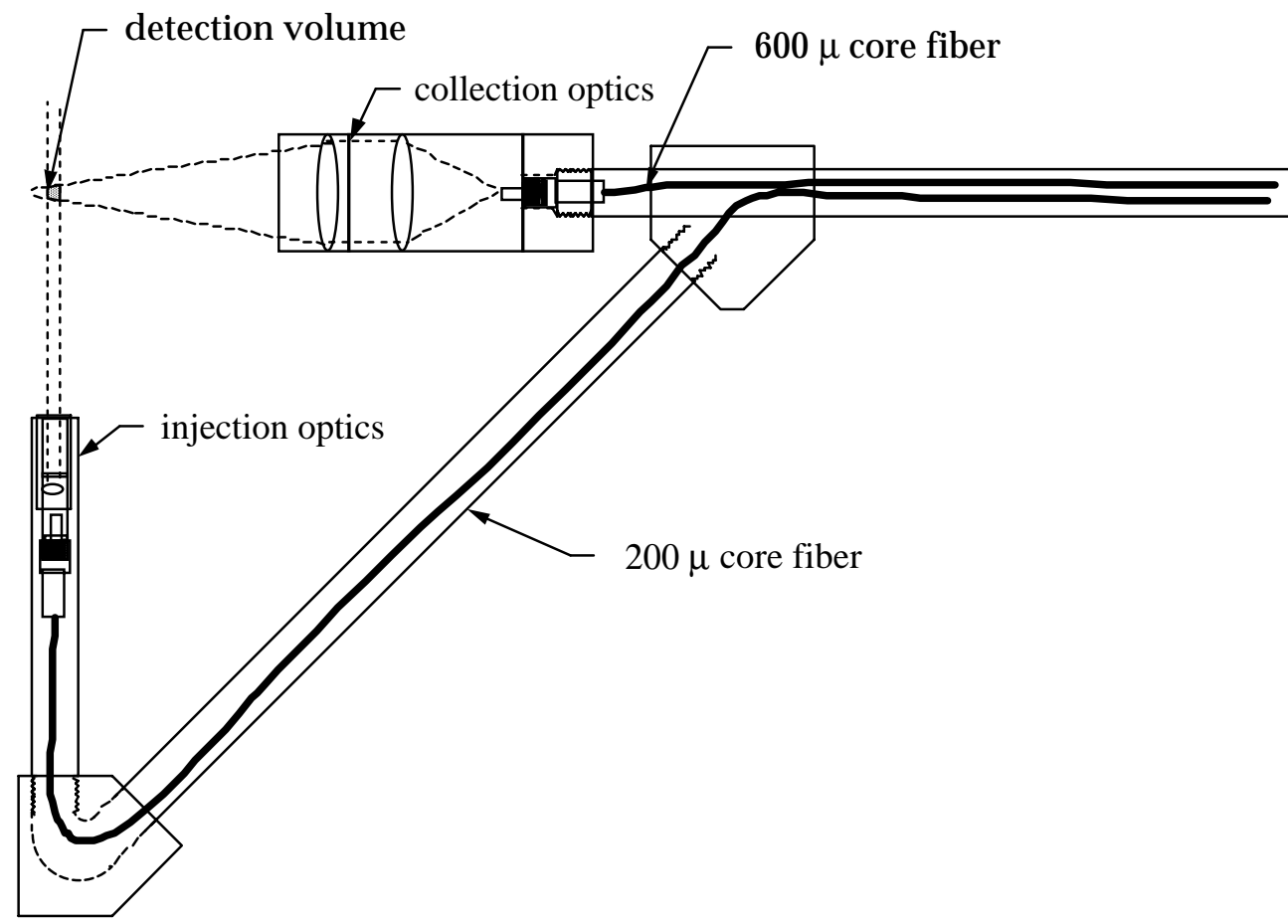

Figure 33: Schematic of the in-situ LIF probe. The laser light $(611.5 \mathrm{~nm})$ is injected into the plasma through the dog-leg and the emission $(461.1 \mathrm{~nm})$ is focused with lenses into a $600 \mu$ core fiber. The probe can move radially and also rotate about the shaft axis.

\subsubsection{Line Broadening Mechanisms}

Spectral lines always have some finite width due to a variety of linewidth broadening mechanisms. Such mechanisms include: natural broadening, Doppler broadening, pressure broadening, Zeeman broadening, power broadening and instrumental broadening.

\subsubsection{Natural Linewidth ${ }^{73}$}

The Heisenberg uncertainty principle is used to determine the natural linewidth of a transition between two levels. For an excited state, $E_{i}$, with a mean lifetime of $\tau_{i}$, the uncertainty is given by: 


$$
\Delta E_{i}=(2 \pi)^{-1} h / \tau_{i}
$$

If the lower level is not the ground state, but another excited state, $E_{k}$, with a mean lifetime of $\tau_{k}$, the uncertainties of both levels contribute to the total uncertainty,

$$
\Delta E=\Delta E_{i}+\Delta E_{k}
$$

Rewriting equation (70) in terms of the uncertainty in the angular frequency and including transitions from any level to either of the two levels of interest leads to

$$
\Delta \omega_{i k}=\sum_{m} \mathrm{~A}_{m i}+\sum_{n} \mathrm{~A}_{n k},
$$

where $i$ and $k$ are the levels of interest, $m$ and $n$ are all levels excluding the level of interest, and $\mathrm{A}_{m i}$ and $\mathrm{A}_{n k}$ are the Einstein coefficients for each transition. Since in this case the lower $3 d^{2} G_{9 / 2}$ state is a metastable state, all of the transitions originating from this level have a very small spontaneous transition probability when compared to a transition originating from the upper $4 p^{2} F_{7 / 2}$. Therefore, the total uncertainty in the angular frequency for this atomic transition is given by:

$$
\Delta \omega_{i k} \approx \sum_{m} \mathrm{~A}_{m i}
$$

The Einstein coefficients for the argon ion levels used for LIF are tabulated ${ }^{73}$ and the uncertainty in the angular frequency is approximately

$$
\Delta \omega_{i k} \cong 1.18 \times 10^{6} \mathrm{~s}^{-1}
$$

In terms of wavelengths,

$$
\therefore \Delta \lambda_{\text {natural }} \cong 2.33 \times 10^{-5} \mathrm{~nm}
$$

\subsubsection{Doppler Broadening ${ }^{73}$}

Doppler broadening is caused by the random thermal motion of a radiating or absorbing atom or ion. If the velocity component of the radiating particle parallel to the direction of observation is $\mathrm{v}$, then the frequency is shifted due to the Doppler effect by the amount: 


$$
\Delta v= \pm v_{0} \mathrm{v} / \mathrm{c}
$$

where $v_{0}$ is the unshifted frequency. Assuming that the particle motion is purely thermal in nature, a Maxwellian distribution function can be used for the emitters/absorbers.

$$
f(\mathrm{v})=\sqrt{\frac{m_{i}}{2 \pi k_{B} T_{i}}} e^{-\left[m_{i}\left(\mathrm{v}-\mathrm{v}_{o}\right)^{2} / 2 k_{B} T_{i}\right]},
$$

where $T_{i}$ is the ion temperature and $\mathrm{v}_{o}$ is the average ion velocity. The light intensity at a particular frequency is proportional to the number of particles at that frequency, i.e. $I(v) d v \propto f(\mathrm{v}) d \mathrm{v}$, where $I(v)$ is the line shape. For a Maxwellian collection of emitters/absorbers, the Doppler-broadened line shape is: ${ }^{61}$

$$
I(v)=I\left(v_{o}\right) e^{\left[-\left(v-v_{o}\right)^{2} c^{2} / 2 v_{\mathrm{th}}^{2} v_{o}^{2}\right]}
$$

where $\mathrm{v}_{t h}^{2}=k_{B} T / m_{i}$ and $v_{o}$ is the frequency corresponding to the center velocity of the ion distribution. Setting the exponential term to $1 / 2$ yields the full width at half maximum (FWHM) of the linewidth:

$$
\Delta v_{1 / 2}=v_{0}\left(\mathrm{v}_{t h} / c\right) \sqrt{2 \ln 2}
$$

Rewriting this in terms of temperature:

$$
\Delta v_{1 / 2}=\left(v_{0} / c\right)\left(2 k_{B} T_{i} / m\right) \sqrt{2 \ln 2}
$$

and, in terms of wavelengths:

$$
\Delta \lambda_{1 / 2}=\left(\lambda_{0} / c\right)\left(2 k_{B} T_{i} / m\right) \sqrt{2 \ln 2} .
$$

For room temperature $\left(T_{i}=0.03 \mathrm{eV}\right)$ ions,

$$
\Delta \lambda_{\text {dop }} \approx 1 \times 10^{-3} \mathrm{~nm}
$$

\subsubsection{Pressure Broadening ${ }^{73}$}

Pressure broadening includes the effects of collisions with neutral particles (van der Waals broadening), resonance interaction between like particles (resonance broadening) and collisions with charged particles (Stark broadening). The first two mechanisms are important for weakly ionized plasmas $(<1 \%$ ionized $),{ }^{74}$ while the last is important for 
highly ionized, high-density plasmas. HELIX is a highly ionized, high-density plasma source, so only Stark broadening might have a significant effect on the linewidth. LEIA, though slightly less ionized than HELIX, is still ionized enough to ignore all pressure broadening except for Stark broadening. The full width at half maximum (FWHM) associated with the Stark effect is given by: ${ }^{75}$

$$
\Delta \lambda_{\mathrm{s} 1 / 2} \approx 2 \times 10^{-16} \omega n_{e}\left[1+1.75 \times 10^{-4} n_{e}^{1 / 4} \alpha\left(1-1.02 \times 10^{-3} n_{e}^{1 / 6} T_{e}^{-1 / 2}\right)\right] .
$$

Where $\omega$ is the electron impact half width parameter, $\alpha$ is the ion broadening parameter, $n_{e}$ is the electron density and $T_{e}$ is the electron temperature. The parameters $\omega$ and $\alpha$ have been tabulated for numerous $\mathrm{Ar}$ ion transitions as a function of electron temperature. ${ }^{75}$ Assuming $T_{e}=5 \mathrm{eV}, n_{e}=10^{14} \mathrm{~cm}^{-3}$ and approximating values for $\alpha$ and $\omega,{ }^{73}$ the Stark broadening is approximately:

$$
\Delta \lambda_{\text {stark }} \approx 1.16 \times 10^{-5} \mathrm{~nm}
$$

\subsubsection{Zeeman Broadening ${ }^{73}$}

The interaction of the magnetic moment of an electron with an applied magnetic field results in the splitting of an observed spectral line. The "normal" Zeeman effect occurs when a singlet line splits into three polarized components. The "anomalous" Zeeman effect occurs when a line is split into a group of four or more components.

When a spectral line splits in a magnetic field, the Zeeman components are polarized either parallel or perpendicular to the magnetic field. The parallel components are referred to as $\pi$ components, or $\pi$ lines, and the perpendicular components are referred to as $\sigma$ components, or $\sigma$ lines. The Zeeman components are always shifted symmetrically about the position of the undisplaced line. For the "normal" Zeeman effect, the three components arising from a line of frequency $f$, have frequencies of $f, f+\Delta f$, and $f-\Delta f$. The undisplaced line is the $\pi$ line and the two displaced lines are the $\sigma$ lines. The $\pi$ line is linearly polarized along the magnetic field direction and the $\sigma$ lines are circularly polarized perpendicular to the magnetic field. The two $\sigma$ components rotate in opposite directions. 
The number of energy levels, or degree of splitting, obtained in a magnetic field depends on the total angular momentum value $(2 J+1)$, of the transition states. $J=1$ gives rise to the normal Zeeman effect. In general, the energy difference between the different lines due to the interaction between the magnetic moment and the magnetic field, $B$, is:

$$
\Delta E=\mu_{B} B g_{j} M_{j},
$$

where, $\mu_{B}=e h\left(4 \pi m_{e}\right)^{-1}$ is the Bohr magneton, $M_{j}$ is the magnetic orbital quantum number, $B$ is the magnetic field in Gauss, and $g_{j}$ is the Lande factor. The Lande factor is given by:

$$
g_{j}=1+\{[J(J+1)+S(S+1)-L(L+1)] / 2 J(J+1)\}
$$

where $J$ is the total angular momentum, $S$ is the spin angular momentum, and $L$ is the orbital angular momentum for the electron energy level. Thus, the energy difference between two levels is:

$$
\Delta E=\mu_{B} B\left(g_{1} M_{1}-g_{2} M_{2}\right)
$$

Rewriting this expression in terms of wavelength

$$
\Delta \lambda=\mu_{B} \lambda^{2} B(c h)^{-1}\left(g_{1} M_{1}-g_{2} M_{2}\right) .
$$

Calculation of each of the terms of equation (87) for the appropriate argon ion parameters is described in reference 73 . For a field of $100 \mathrm{G}$, the largest Zeeman broadening due to the splitting of the $\sigma$ lines is approximately

$$
\Delta \lambda_{\text {zee }}=2.6 \times 10^{-5} \mathrm{~nm} .
$$

\subsubsection{Power Broadening ${ }^{73}$}

Power broadening occurs when the stimulated photon emission rate equals the photon absorption rate and both are greater than the spontaneous emission rate. When saturation occurs, the power residing in the wings of the laser becomes important, resulting in photon absorption far from the laser central line. ${ }^{76}$ Goeckner and Goree developed empirical equations that can be used to estimate the effects of power broadening. ${ }^{77}$ Goeckner and Goree's experiments used a $1 \mathrm{GHz}$ bandwidth, pulsed laser (17 ns) tuned to the same argon transition used for LIF in HELIX and LEIA argon plasmas. Since the 
linewidth of the laser described in section 3.2 is approximately $1 \mathrm{MHz}$, significantly less broadening is expected than with a $1 \mathrm{GHz}$ linewidth laser. Assuming the laser beam has a diameter of about $2 \mathrm{~mm}$ and a maximum power of $100 \mathrm{~mW}$, an upper bound for the effects of power broadening is given by

$$
\Delta \lambda_{\text {power }} \leq 1 \times 10^{-4} \mathrm{~nm} .
$$

\subsubsection{Instrumental Broadening ${ }^{73}$}

There are two types of instrumental broadening; one associated with the laser linewidth, and one associated with the dispersion of the light detection system. The emission light is measured with a passband filtered $( \pm 1 \mathrm{~nm})$ photomultiplier tube. Thus, there is no dispersion in the collection system. The laser bandwidth for the Coherent 899 Ring laser is less than $1 \mathrm{MHz}$, which in terms of wavelengths is

$$
\Delta \lambda_{\text {laser }} \leq 1.25 \times 10^{-6} \mathrm{~nm}
$$

for a central wavelength of $611.53 \mathrm{~nm}$.

\subsubsection{Comparison of Broadening Mechanisms}

Because the Doppler FWHM is used to determine the ion temperature in these experiments, any of the other mechanisms that artificially broaden the LIF lineshape must be accounted for before the ion temperature can be estimated. 
Table 5: Approximate linewidths associated with different lineshape broadening mechanisms listed in order of significance.

\begin{tabular}{|c|c|}
\hline Broadening Mechanism & FWHM value (nm) \\
\hline Doppler Broadening & $\sim 1.0 \times 10^{-3}$ \\
\hline Zeeman Broadening $(\sigma$ lines) & $\leq 2.6 \times 10^{-4}$ \\
\hline Power Broadening & $<1.0 \times 10^{-4}$ \\
\hline Pressure Broadening & $\sim 1.2 \times 10^{-5}$ \\
\hline Natural Linewidth & $\sim 2.3 \times 10^{-5}$ \\
\hline Instrumental Broadening & $\sim 1.3 \times 10^{-6}$ \\
\hline
\end{tabular}

As shown in Table 5, most of the possible lineshape broadening mechanisms have a much smaller FWHM value than Doppler broadening. However, in HELIX, Zeeman broadening can be as much as $25 \%$ of the Doppler broadening. As mentioned previously, a linear polarizer followed by a quarter waveplate is used to select a single circular polarization for parallel LIF measurements. For perpendicular LIF measurements, a linear polarizer in the collection optics is used to select the linearly polarized $\pi$ lines. The power broadening is an extreme overestimation and experimental tests have show that the laser power can be changed by a factor of three and the measured distribution function is not affected (Figure 34). A typical LIF trace is shown in Figure 35. The LIF signal is well fit by a single Maxwellian distribution function. 


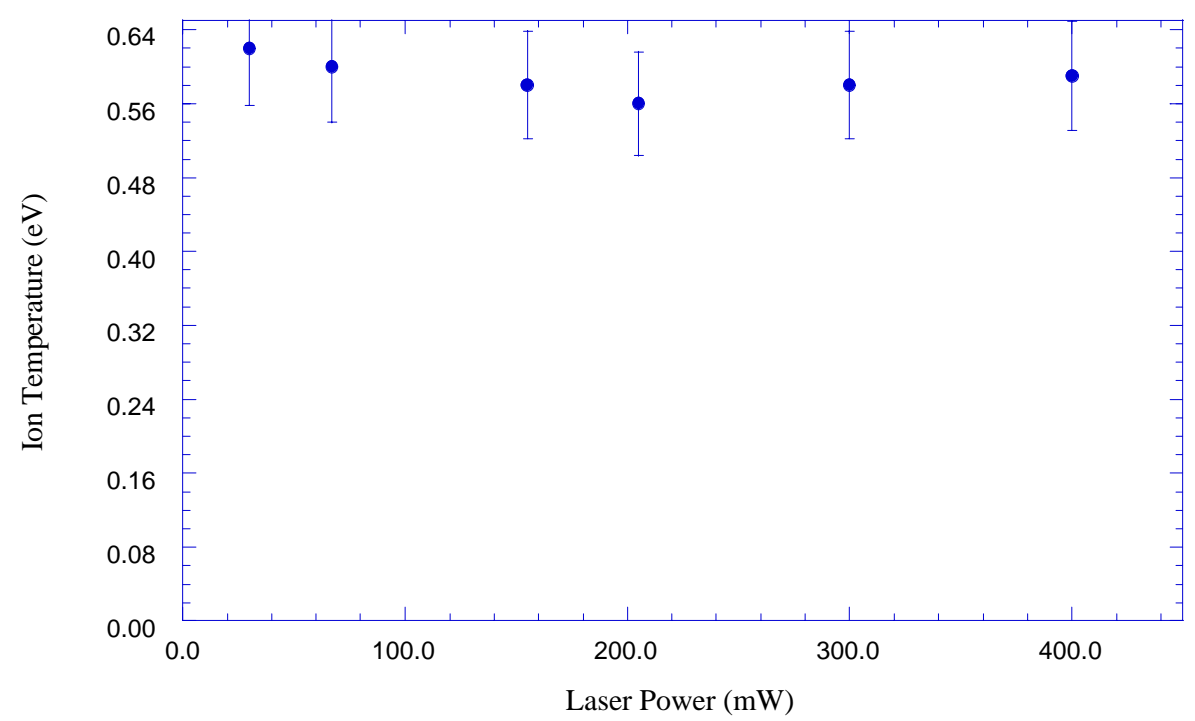

Figure 34: Argon ion temperature as a function of dye laser power with $10 \%$ error bars. The data were taken at a pressure of $7.1 \mathrm{mTorr}$, rf frequency of $8.5 \mathrm{MHz}$, rf power of 600 $\mathrm{W}$ and $B \sim 1 \mathrm{kG}$.

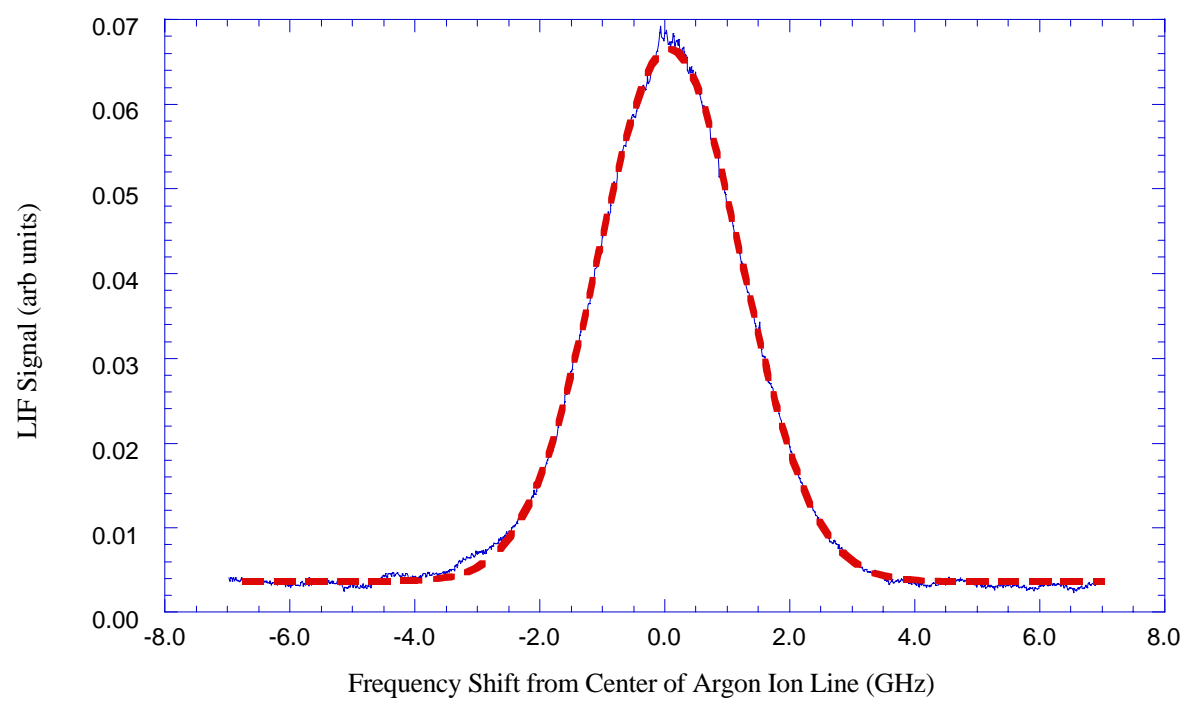

Figure 35: A typical LIF trace (solid line) for a perpendicular measurement in LEIA. The ion temperature from the fit (dashed line) is $0.21 \mathrm{eV}$ in this case. 


\subsection{Magnetic Probe}

\subsubsection{Magnetic probe construction and calibration}

A standard magnetic sense coil is made from a loop, or loops, of wire. If the magnetic field passing through the loop of wire is time-dependant, then, from Faraday's Law, a voltage will be induced around the loop. If the voltage is measured over a period of time, a time series record of the signal can be recorded. Using Fourier analysis, spectral features can then be identified and examined. Comparing the signals from two coils provides a measurement of the phase shift between the two coils. The phase shift at a particular frequency divided by the distance between the probes yields the wavenumber, $k$, of the magnetic fluctuation.

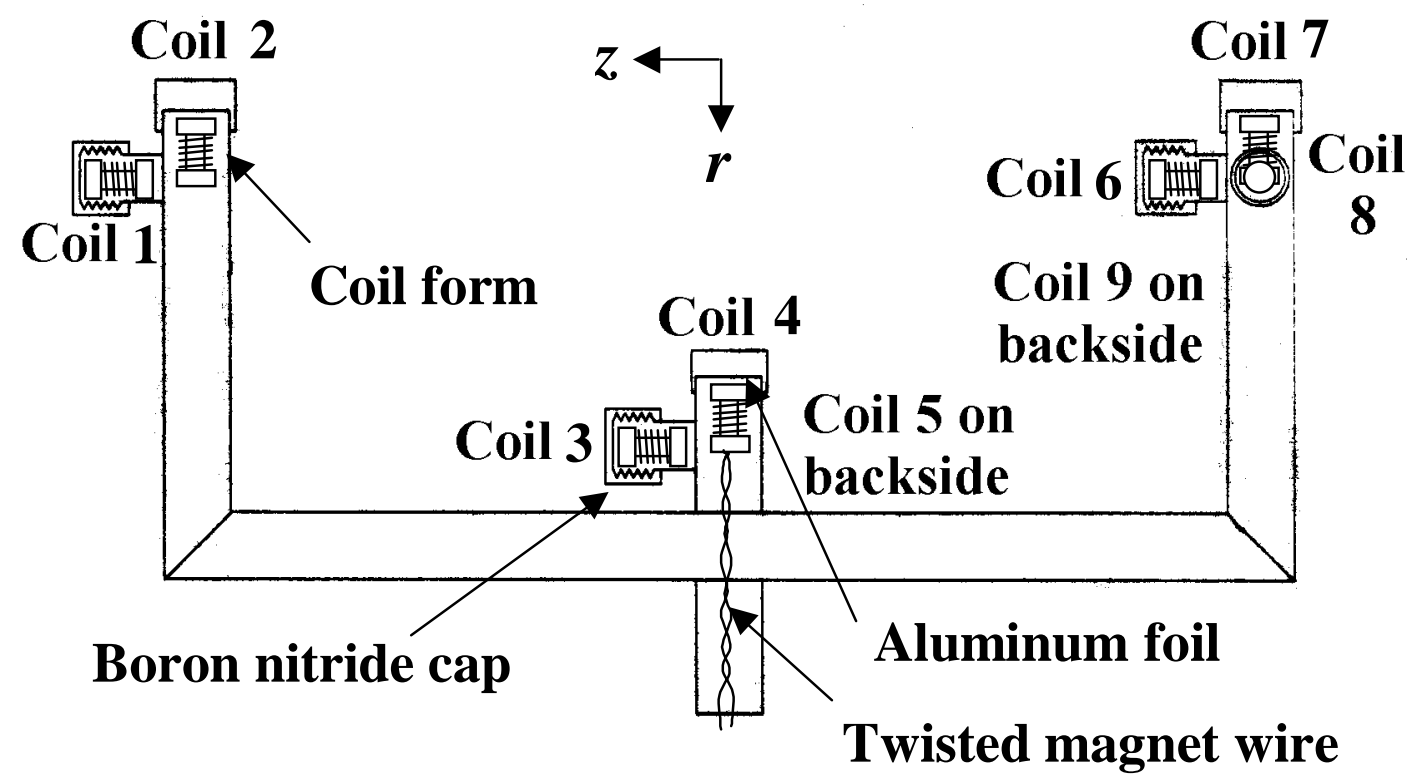

Figure 36: Magnetic probe diagnostic used in LEIA. There are two coils not seen in this view, one on the backside of the middle leg and one on the backside of the right leg. Each coil is wound with nominally two hundred turns of 35 gauge copper magnet wire on a barbell shaped piece of boron nitride. The shafts are stainless steel and each coil is capped with a thin piece of aluminum foil and a boron nitride cover. 
A schematic of the magnetic probe used in LEIA is shown in Figure 36. The design of the probe allows three-dimensional measurements of the wavenumber spectrum. The coils are identified as coils 1-10. Coils 1, 3 and 6 are along the $z$-direction, coils 2, 4, and 7 are along the $r$-direction, and coils 5, 8, and 9 are in the $\theta$-direction. As coil 5 was defective, no data was available from coil 5. Coil 10 was a single radial coil placed on another probe farther upstream from the other 9 coils.

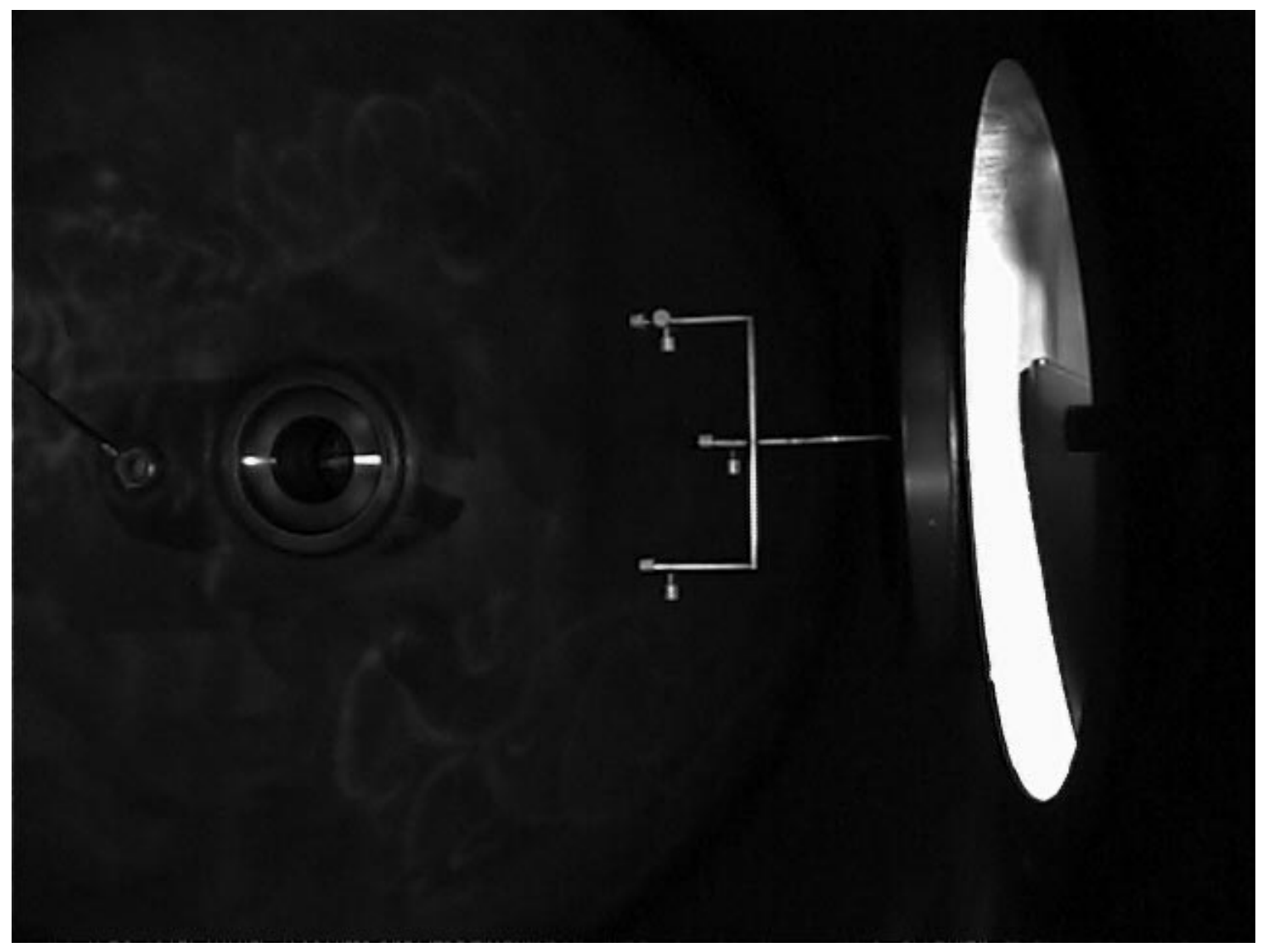

Figure 37: A picture of the magnetic fluctuation probe inside LEIA.

The magnetic probe was calibrated to determine the inherent phase shift in each coil due to the length of wire, the Tektronix 4780 signal conditioner and the Tektronix 4244 digitizer. A measurement of the inherent phase shift is required to ensure that any measured phase shifts between two coils are real and not electronic artifacts. A solenoidal 
coil driven with a HP function generator was used to generate a time-varying magnetic field for calibrating the coils. Each magnetic fluctuation sense coil was placed within the solenoid and the solenoid was then ramped in frequency, starting at $100 \mathrm{~Hz}$ and ending at 100,000 Hz. At each frequency step, the amplitudes of the current through the solenoid (the reference signal) and the signal detected by the magnetic sense coil were measured. The phase shift between the two signals at each frequency was used as a measure of the inherent phase shift in each coil relative to the calibration coil. The relative amplitudes of the signals were also used to determine the effective area of the coils as a function of frequency. The voltage induced in the magnetic sense coil is given by:

$$
V_{\text {coil }}(t)=N A \dot{B}(t),
$$

where $N$ is the number of turns on each coil, $A$ is the area of each coil and $\dot{B}$ is the magnitude of the time derivative of the magnetic field. Since $B=B_{o}(f) \sin (2 \pi f t)$, $\dot{B}=2 \pi f B_{o}(f) \cos (2 \pi f t) . \quad B_{o}(f)=I_{\text {source }}(f) \mu_{o} n$, where $I_{\text {source }}(f)$ is the frequency dependent output current of the function generator and $n$ is the turns per unit length of the solenoid. The relationship between $B_{o}(f)$ and $I_{\text {source }}(f)$ as a function of frequency was measured with a Walker Scientific model MG-50 gaussmeter. Therefore, at anytime $t$,

$$
V_{\text {coil }}(t)=2 \pi N A f B_{o} \cos (2 \pi f t) .
$$

In terms of the peak values, the effective sense coil area as a function of the frequency is $N A=V_{p t p} / 4 \pi f B_{o}$. Plots of the area and phase shift of each coil versus frequency are shown in Figure 38. Each coil was nominally wrapped with 200 turns of 35 gauge copper magnet wire. 

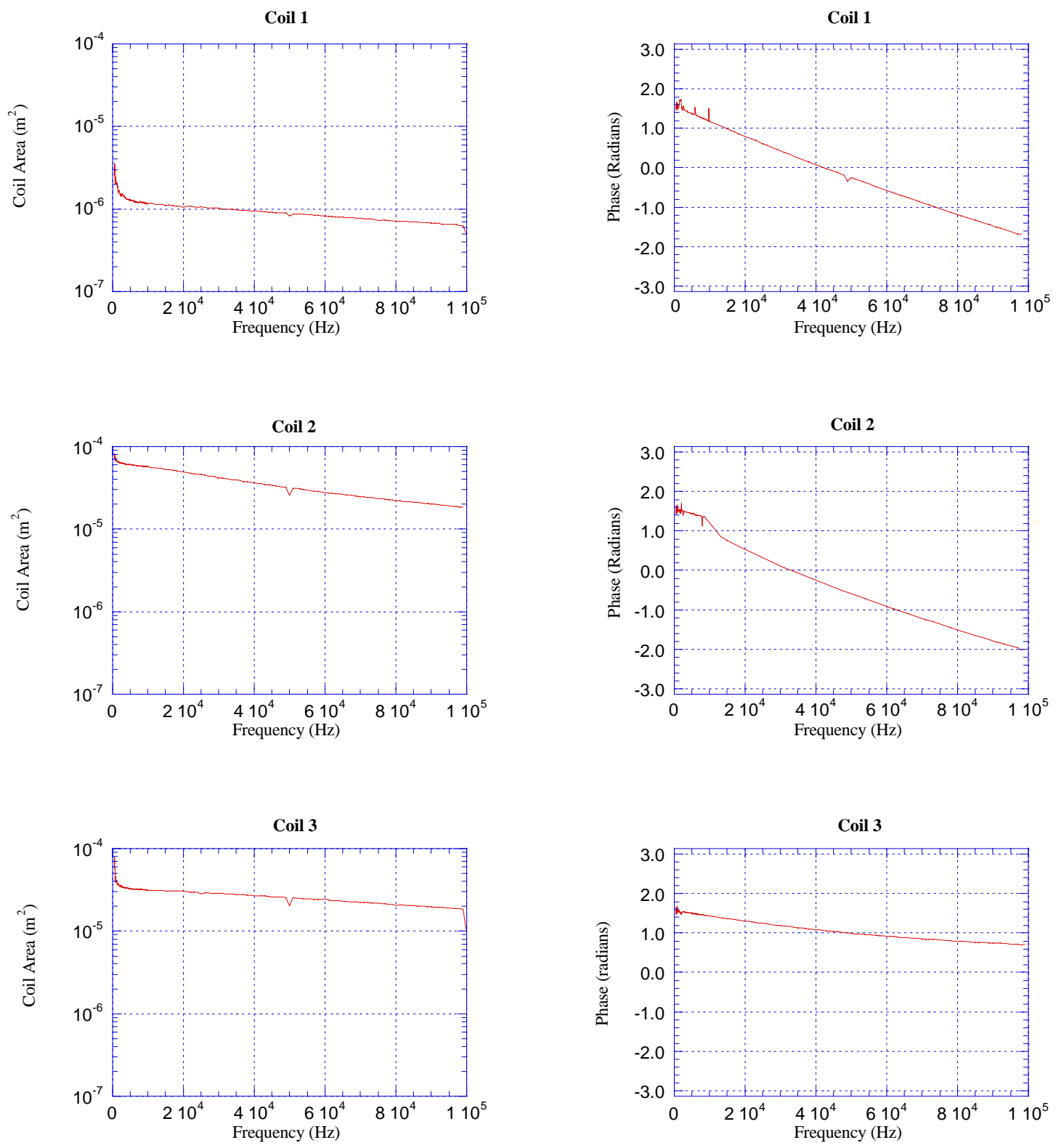
Coil 4

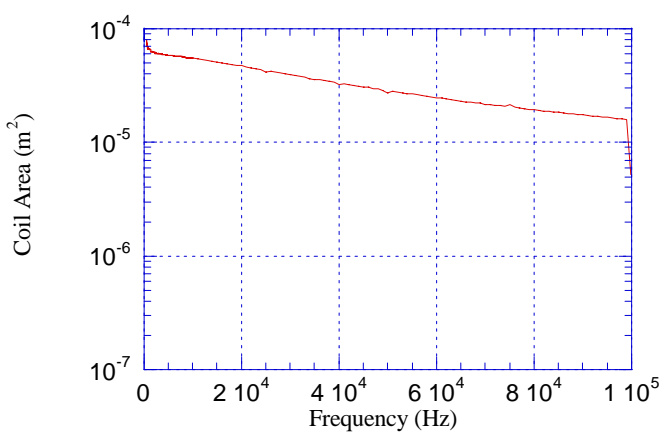

Coil 6

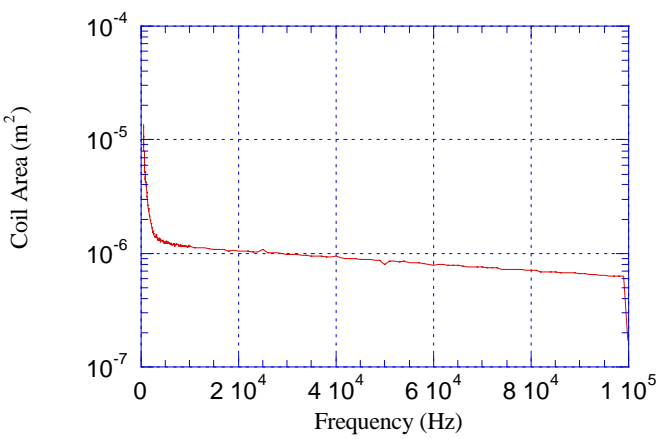

Coil 7

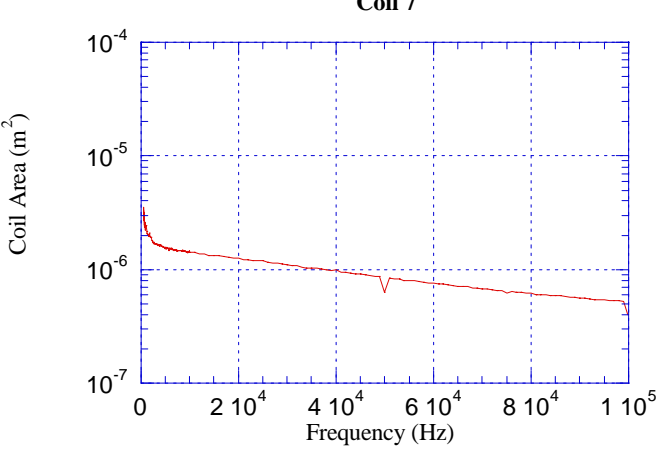

Coil 8

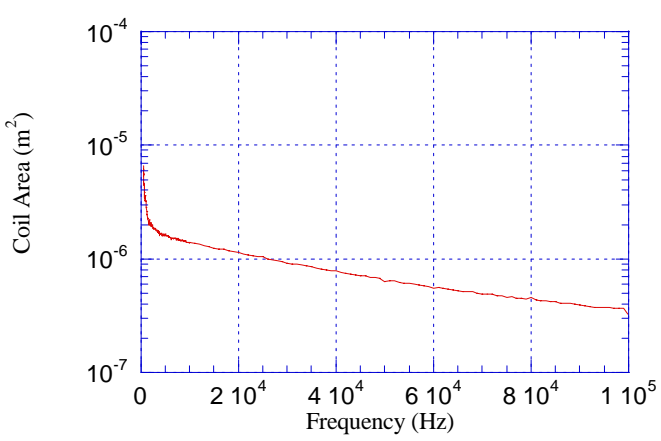

Coil 4

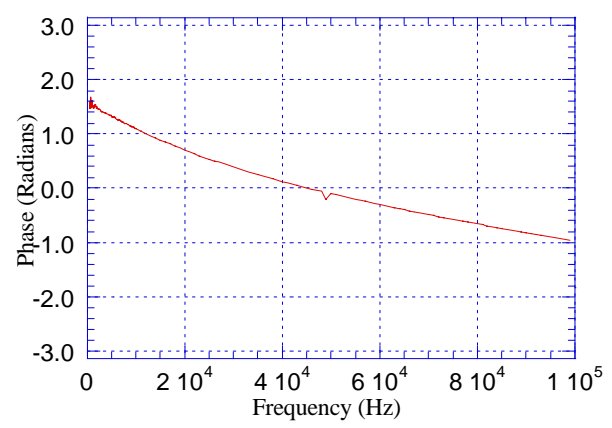

Coil 6

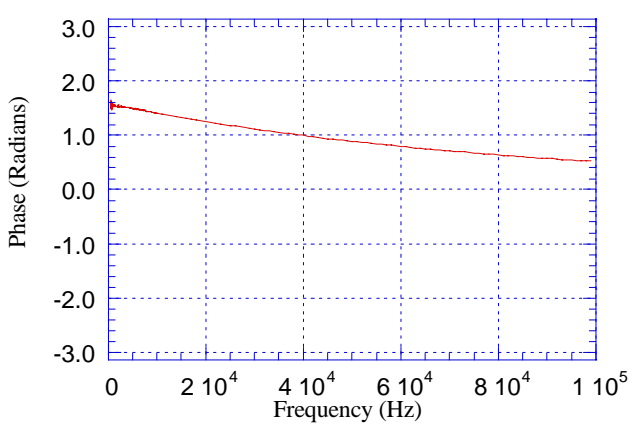

Coil 7

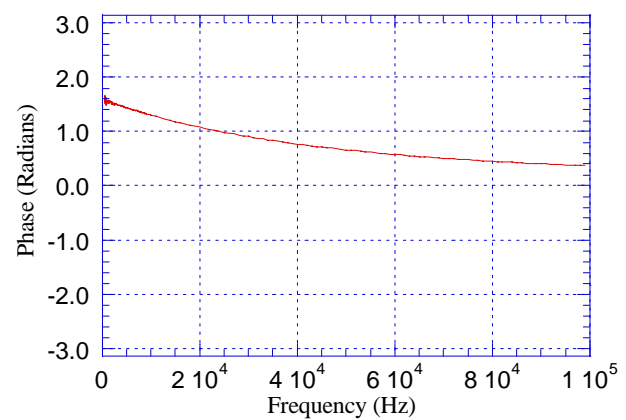

Coil 8

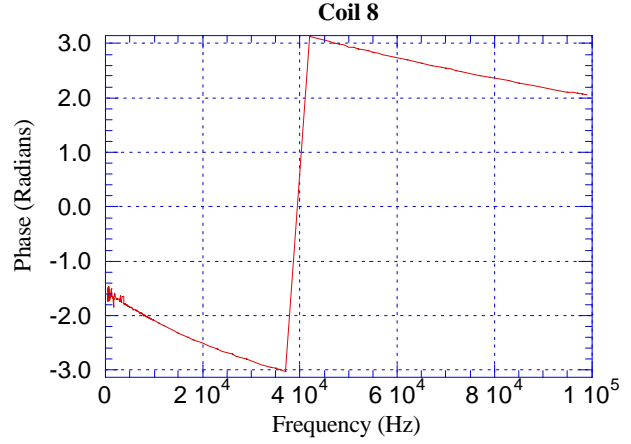



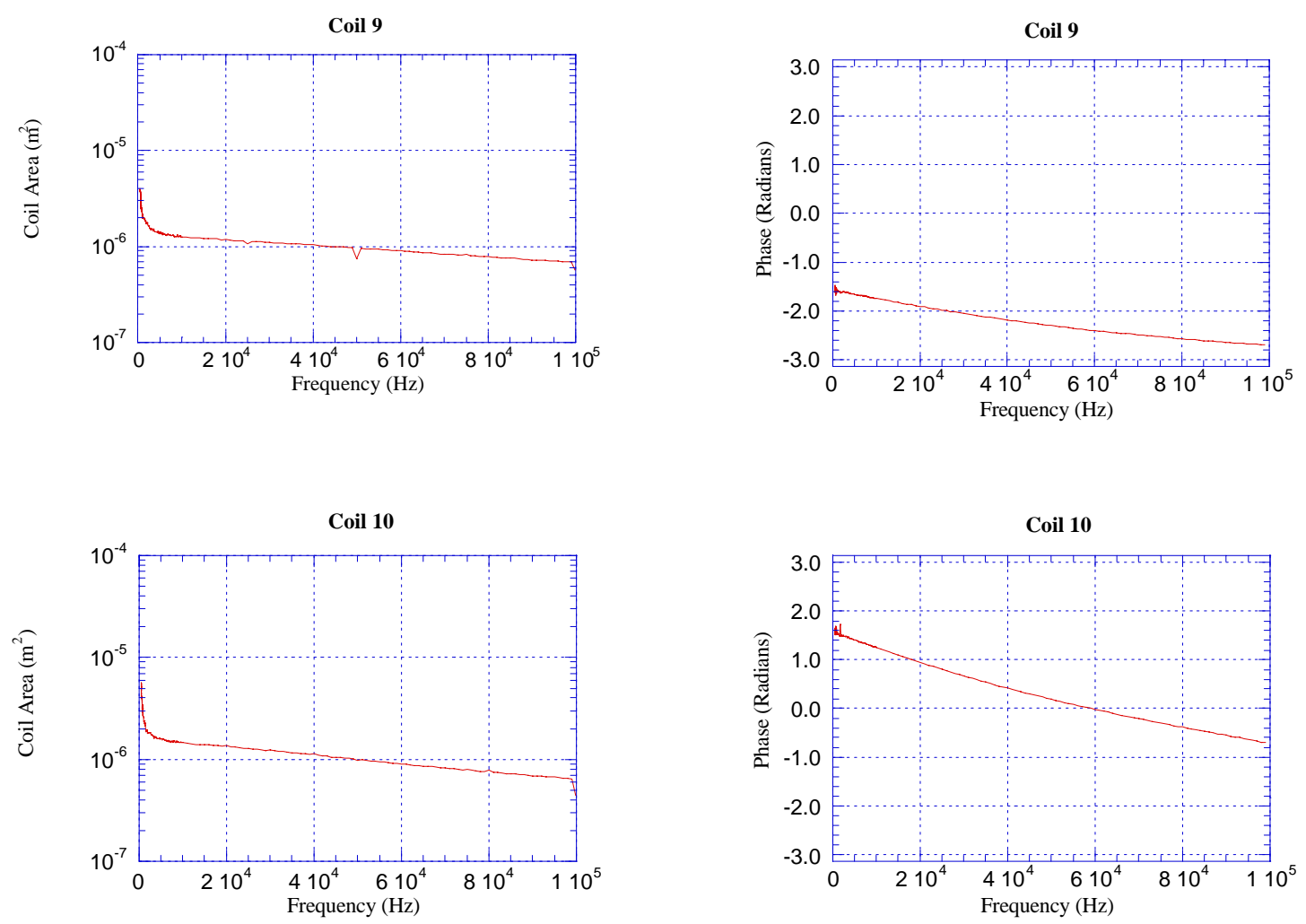

Figure 38: Plots of the total coil area $(N A)$ and relative phase shift of all the magnetic sense coils.

When the data were being analyzed, it was discovered that the inherent phase difference between some pairs of coils increased significantly as a function of frequency. Although the inherent phase difference of a single coil should vary as a function of frequency, the phase difference between two coils should be relatively constant because the coils are essentially identical. To avoid significant systematic errors in the wavenumber calculations discussed in Chapter 6 , only data from the radial coils $(2,7,10)$ were used. At low frequency, the phase difference between coils 7 and 10 is quite small and the phase difference between 1 and 10 is relatively independent of frequency. 


\subsubsection{Analysis of magnetic probe signals}

Time series from the set of magnetic sense coils provide frequency spectra of the electromagnetic fluctuations parallel and perpendicular to the equilibrium magnetic field. However, the frequency spectra of the waves are not sufficient to characterize the instabilities in LEIA. To clearly identify an instability, the dispersion relation $\omega(k)$ must be determined. Thus, the wavenumber, $k$, associated with each frequency is required. Although such information cannot be determined from a single probe, Beall et al. ${ }^{78}$ devised a method to estimate the wavenumber of a wave from fixed probe pairs.

Each probe time series can be described by a realization, $\phi(x, t)$, where $x$ is the location of the probe. The realization, which occurs over a time interval, $T$, and over a region of space of length $L$, can be Fourier transformed into the form $\phi(k, \omega)$. Transforming first into frequency space,

$$
\Phi(x, \omega)=\int_{-T / 2}^{T / 2} \phi(x, t) e^{-i \omega t} d t
$$

and then wavenumber space,

$$
\Phi(k, \omega)=\int_{-L / 2}^{L / 2} \phi(x, \omega) e^{-i k x} d x
$$

where $\Phi(k, \omega)$ is the amplitude of the sense coil signal as a function of $k$ and $\omega$. Likewise, the inverse Fourier transform to convert $\phi(k, \omega)$ into the physical space and time domains are given by:

$$
\begin{gathered}
\phi(k, t)=\int_{-\omega / 2}^{\omega / 2}(2 \pi)^{-1} \Phi(k, \omega) e^{i \omega t} d \omega \\
\phi(x, t)=\int_{-\omega / 2}^{\omega / 2}(2 \pi)^{-1} \int_{-K / 2}^{K / 2}(2 \pi)^{-1} \Phi(k, \omega) e^{i k x+i \omega t} d \omega d k
\end{gathered}
$$

The power spectrum, or spectral density, of a signal is defined as:

$$
P(x, \omega)=\frac{1}{T}<\Phi^{*}(x, \omega) \Phi(x, \omega)>,
$$

where $*$ donates the complex conjugate and $<>$ denotes an average over the time interval $T$. The power spectrum is essentially the square of the Fourier amplitude for a single coil 
at a single position in space. The power spectrum yields the amplitude of the fluctuating magnetic field as a function of frequency. To determine the power spectrum of the magnetic field, $P(x, \omega)$ must be divided by $\omega$.

The cross power spectrum between two different coils at a specific frequency identifies correlated signals between the two coils and is given by ${ }^{79}$

$$
P_{12}(d, \omega)=\left\langle\Phi_{1}^{*}\left(x_{1}, \omega\right) \Phi_{2}\left(x_{2}, \omega\right)\right\rangle,
$$

where the subscripts 1 and 2 identify which coil, coil 1 or coil 2 , is used and $d$ is the distance between the probes. Since $P_{12}(d, \omega)$ is complex, equation (98) can also be written as ${ }^{79}$

$$
P_{12}(d, \omega)=C_{12}(d, \omega)+i Q_{12}(d, \omega) .
$$

$C_{12}(d, \omega)$ is the real part and is referred to as the in-phase component, the coincident spectral density function or the cospectrum. $Q_{12}(d, \omega)$ is the imaginary part and is referred to as the quadrature component, the quadrature spectral density function or the quadspectrum. $P_{12}(d, \omega)$ can also be expressed in polar coordinates, with an amplitude and a phase: ${ }^{79}$

$$
P_{12}(d, \omega)=\left|P_{12}(d, \omega)\right| e^{i \theta_{12}(\omega)} .
$$

The phase shift between two coils separated by a distance $d$ at some frequency $\omega$ is defined as: ${ }^{78}$

$$
\Theta_{12}(\omega)=\tan ^{-1}\left(Q_{12}(d, \omega) / C_{12}(d, \omega)\right) .
$$

Thus, the sample local wavenumber, $K(\omega)$, is given by:

$$
K(\omega)=\Theta_{12}(\omega) / d
$$

Crudely, $K(\omega)$ is the $k$ value as a function of frequency for a given pair of coils. Given $K(\omega)$, the two-dimensional local wavenumber and frequency spectrum, $\hat{S}_{l}(K, \omega)$ is constructed by summing over possible values of $k$ and $\omega$ and is given by:

$$
\hat{S}_{l}(K, \omega)=\frac{1}{M} \sum_{j=1}^{M} I_{(0, j \Delta K)}[K-K(\omega)] \times \frac{1}{2}\left[P_{1}(d, \omega)+P_{2}(d, \omega)\right]
$$


where $P_{1}(d, \omega)$ and $P_{2}(d, \omega)$ are the power spectra for coils 1 and coils 2 respectively; $K$ is the upper value of the current wavenumber bin, $j \Delta K$; and the indicator function $I_{(0, h)}[x]$ is defined as

$$
I_{(0, h)}[x]=\left[\begin{array}{cc}
1 & 0 \leq x h \\
0 & \text { elsewhere }
\end{array}\right] .
$$

$K(\omega)$ has a range of $\pm \pi / 2 d . \Delta K$, the bin size, is $\pi / 2 d M$, where $M$ is the number of points in the record and $d$ is the distance between the two probes. For a given frequency, $K(\omega)$ is compared to the wavenumber bin. If $K(\omega)$ falls into the wavenumber bin, then the average power spectra of the two coils is used to fill the bin. Otherwise, the bin is filled with a 0 . This process continues until all values of wavenumber and frequency in the two-dimensional array are completed. A graph of $\hat{S}_{l}(K, \omega)$ is then used to identify peaks as a function of $k$ and $\omega$ and the measured $k$ and $\omega$ can then be compared to possible dispersion relations.

To illustrate this technique, a hypothetical single frequency, traveling sine wave of wavelength $\lambda=12.57 \mathrm{~m}$ and frequency $\omega_{o}=37,700 \mathrm{rad} / \mathrm{s}$ measured by two coils a fixed distance apart is examined (Figure 39). The single coil power spectra (Figure 40) for this wave will be delta functions because all the power exists at a single frequency.

$$
\begin{aligned}
& P_{1}\left(x_{1}, \omega\right)=\delta\left(x_{1}, \omega_{o}\right) \\
& P_{2}\left(x_{2}, \omega\right)=\delta\left(x_{2}, \omega_{o}\right)
\end{aligned}
$$

The two coils have a relative phase shift at one specific frequency, which is determined from the cross power spectrum and equations (98) through (101). Dividing the phase shift by the distance yields the local wavenumber at that frequency. Even though there are a multitude of other frequencies, the local wavenumber for all those frequencies is zero in this case because the power spectra are zero at those frequencies.

In other words, the creation of the discrete $\hat{S}_{l}(K, \omega)$ two-dimensional array begins by determining the local wavenumber for some particular frequency and storing the average value of the power spectra of the two coils at that frequency. Then, the next frequency step is examined and the process repeats until all possible $\omega$ s and $k$ 's are covered. Since this test scenario has only a single frequency and a single wavelength, there will only be 
one point in the two dimensional array that is non-zero. If a signal were composed of many frequencies and a single wavelength, there would be a band of non-zero array elements at a single wavenumber. Conversely, a single frequency at multiple wavelengths would appear as a band of non-zero values at a single frequency. As this type of analysis is very sensitive to statistical noise, many time series measurements are performed and the results averaged to improve the signal to noise ratio. The two-dimensional wavenumber spectrum for this example is shown in Figure 41. For the experiments reported here, all calculations are based on fifty or more time series measurements.

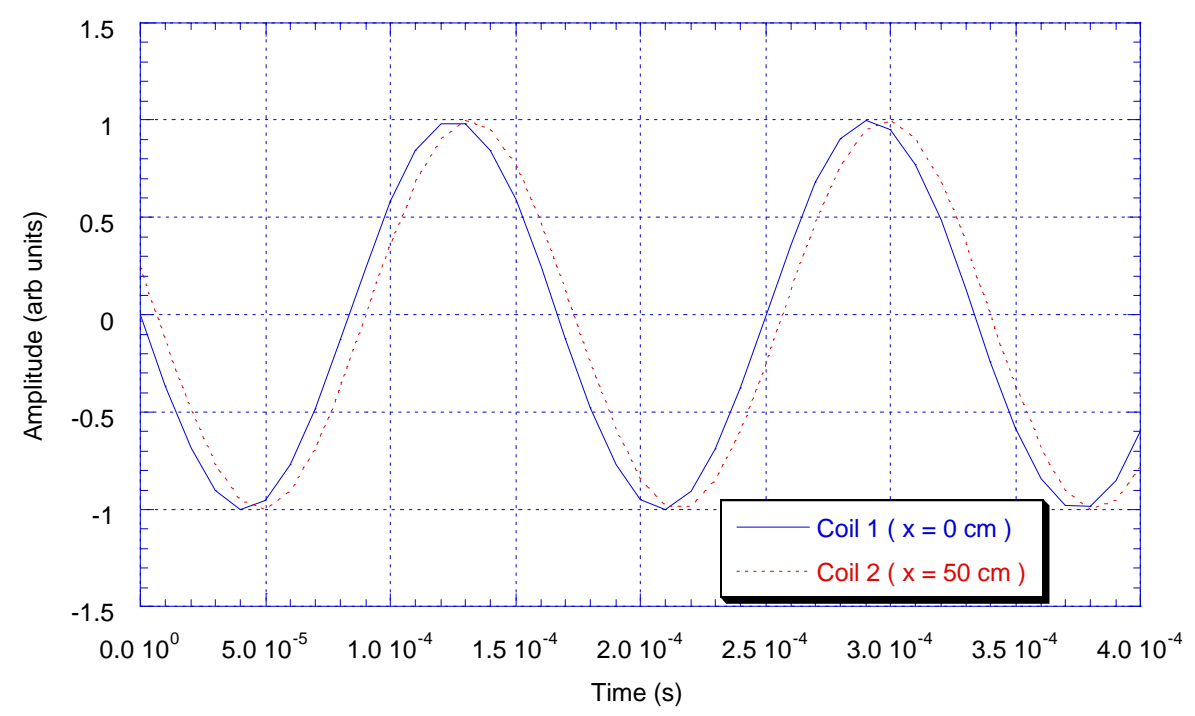

Figure 39: Partial times series from two coils separated by $50 \mathrm{~cm}$. This hypothetical wave has a frequency of $6 \mathrm{kHz}$. 


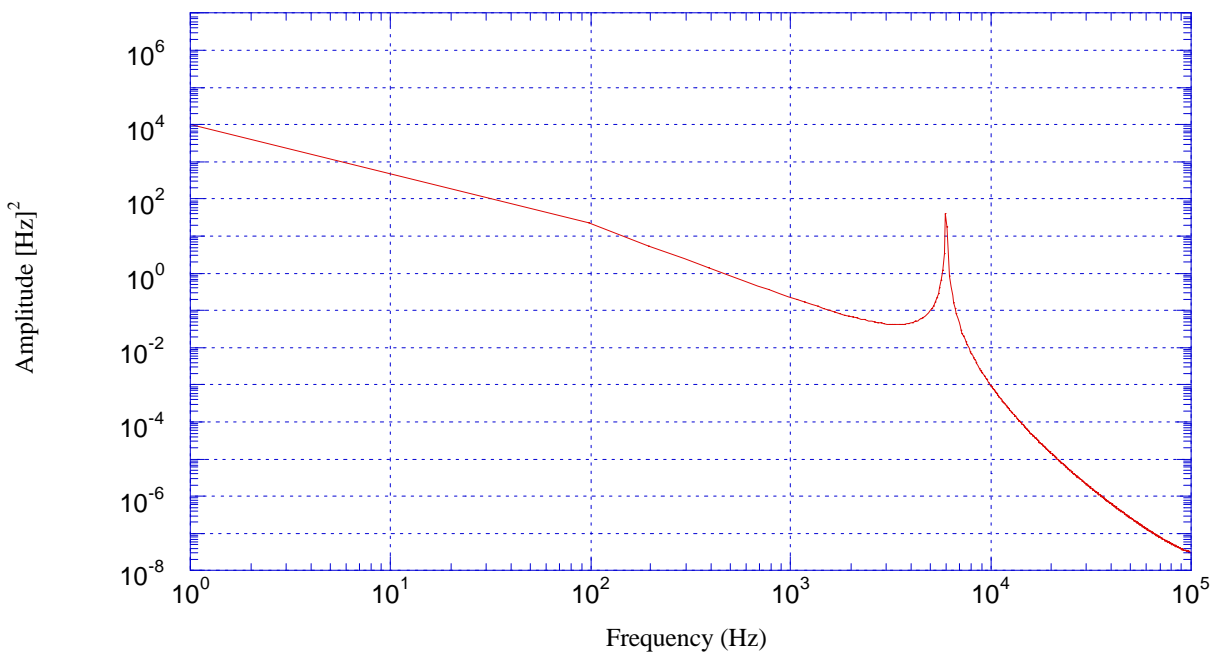

Figure 40: Power spectrum of the test signal. The curve decreases with frequency because the spatial amplitude is divided by the frequency so that the amplitude of the fluctuating quantity is found.

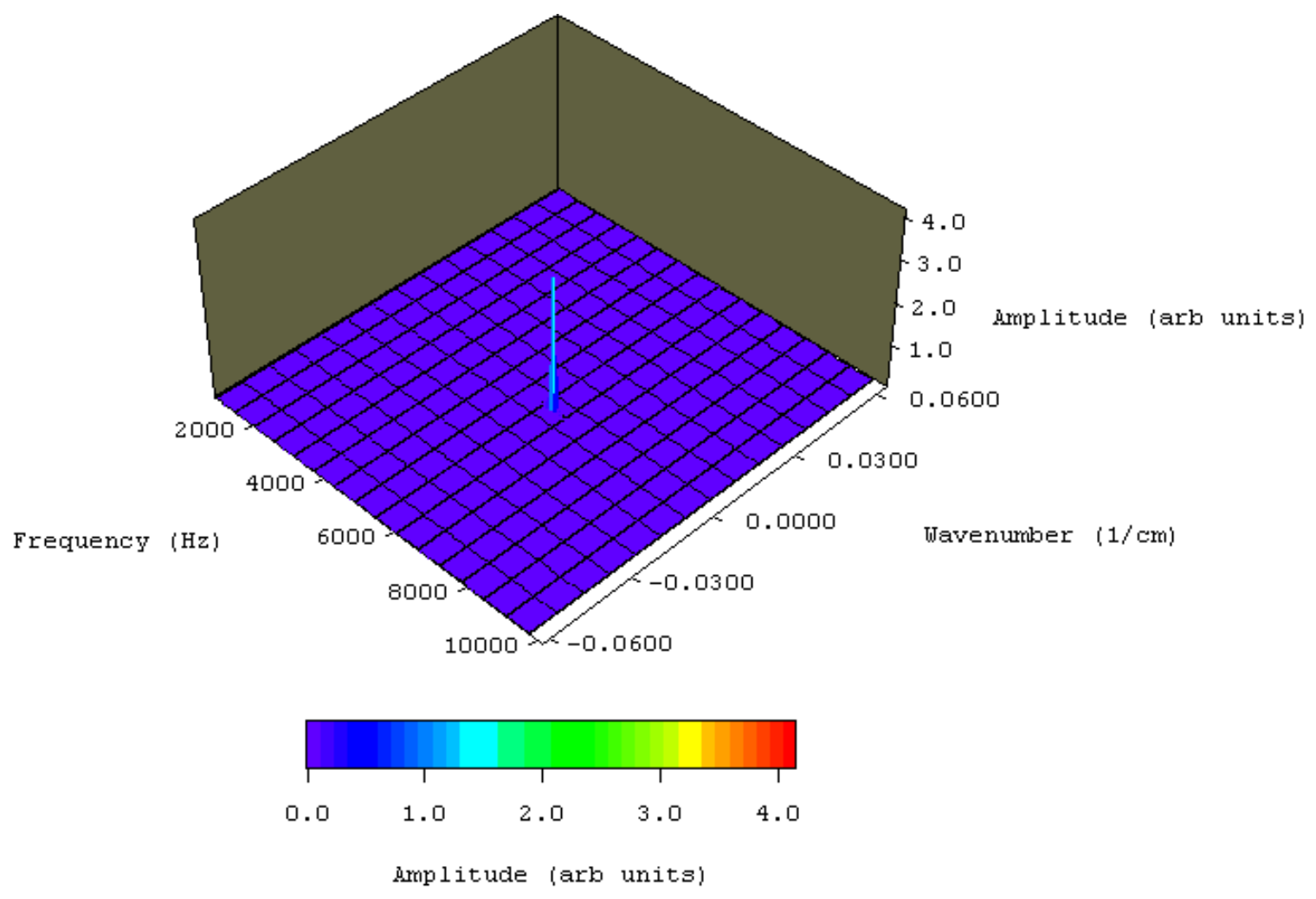

Figure 41: Surface plot of the wavenumber spectrum for the hypothetical case. One large peak is present at $6 \mathrm{kHz}$ and $k=0.05 \mathrm{~cm}^{-1}(\lambda=12.6 \mathrm{~m})$ as expected. 


\section{ELECTROMAGNETIC ION TEMPERATURE ANISOTROPY DRIVEN INSTABILITIES}

Since the 1960's, space plasma phenomena attributed to electromagnetic ion temperature instabilities (EMITA) have been observed in the Earth's magnetosphere and the solar wind. Vela 3 and Mariner 2 measurements of ion temperature anisotropies $^{80,81,82}$ correlated with magnetic fluctuations at frequencies below the ion cyclotron frequency ${ }^{83} 84,85,86$ established a link between such anisotropies and the excitation of electromagnetic instabilities. Analysis of these observations led to the development of theories for the ion firehose, ${ }^{87}$ the mirror, and the ion cyclotron anisotropy instabilities. The launch of spacecraft with more capable ion instruments in the late 1980's, in particular the AMPTE series of spacecraft, enabled researchers to measure three-dimensional ion distributions in both the solar wind and the magnetosheath. Particularly in the magnetosheath, ion velocity-space distributions during periods of enhanced low frequency electromagnetic fluctuations are observed to be biMaxwellian, i.e., the ion distribution function can be represented by a Maxwellian distribution in the direction perpendicular to the background magnetic field of one temperature, $T_{i \perp}$, and a Maxwellian distribution function in the direction parallel to the background magnetic field of a different temperature, $T_{i \|}$. A striking feature of the AMPTE magnetosheath data is an inverse correlation of the measured ion temperature anisotropy, $T_{i \perp} / T_{i \|}$, and the local plasma ion beta. Based on a combination of linear Vlasov theory and hybrid-kinetic simulations, theorists have suggested that the inverse correlation of ion temperature anisotropy and the local plasma beta can be explained by the action of anisotropy driven instabilities.

Temperature anisotropies can provide a source of free energy for instability growth in space plasmas. There are three main types of EMITA instabilities depending on the value of the ratio of parallel and perpendicular ion temperatures. They are the ion firehose $\left(T_{\|}>T_{\perp}\right){ }^{87}$ the ion cyclotron ${ }^{87}$ - also called the Alfvén ion cyclotron instability $\left(T_{\perp}>T_{\|}\right){ }^{108}$ and the mirror instability $\left(T_{\perp}>T_{\|}\right) .{ }^{88,89}$ Each of the anisotropy driven instabilities acts to reduce the ion temperature anisotropy. The instabilities are 
gyroresonant with the ions and induce velocity-space diffusion that isotropizes the ion distribution. The predicted thresholds, propagation directions, frequencies, and wavelengths of all three instabilities are distinct. In space, both the fluctuation spectra and propagation direction are used to identify the type of instability, given local anisotropy measurements. The main advantages of a laboratory experiment over spacecraft measurements are the degree of diagnostic access to the plasma, e. $g$., the capability of measuring wavelengths, and varying the plasma parameters in a controlled and reproducible fashion.

\subsection{Linear Vlasov Solution}

The linear growth rates of the different EMITA instabilities can be calculated using the first-order Vlasov equation. Following the analysis of Gary ${ }^{91}$ and assuming the primary ions in the solar wind and near-earth space environment are protons, that the plasma is collisionless, and that the ions can be described by a bi-Maxwellian distribution function, the collisionless Vlasov equation is: ${ }^{91}$

$$
\frac{\partial f_{j}(\overrightarrow{\mathrm{x}}, \overrightarrow{\mathrm{v}}, t)}{\partial t}+\overrightarrow{\mathrm{v}} \cdot \vec{\nabla} f_{j}(\overrightarrow{\mathrm{x}}, \overrightarrow{\mathrm{v}}, t)+\frac{q}{m_{j}}(\overrightarrow{\mathrm{E}}(\overrightarrow{\mathrm{x}}, t)+\overrightarrow{\mathrm{v}} \times \overrightarrow{\mathrm{B}}(\overrightarrow{\mathrm{x}}, t)) \cdot \nabla_{\mathrm{v}} f_{j}(\overrightarrow{\mathrm{x}}, \overrightarrow{\mathrm{v}}, t)=0,
$$

where $f_{j}$ is the distribution function for the $\mathrm{j}^{\text {th }}$ species and the $0^{\text {th }}$ order bi-Maxwellian distribution function, $f_{j}^{(0)}$, is:

$$
f_{j}^{(0)}\left(\mathrm{v}_{\|}, \mathrm{v}_{\perp}\right)=\frac{n_{j}}{\left(2 \pi \mathrm{v}_{\mathrm{j}}^{2}\right)^{3 / 2}} \frac{\mathrm{T}_{\| \mathrm{j}}}{\mathrm{T}_{\perp \mathrm{j}}} e^{-\left(\frac{\mathrm{v}_{\|}^{2}}{2 \mathrm{v}_{\mathrm{j}}^{2}}-\frac{\mathrm{v}_{\mathrm{x}}^{2}+\mathrm{v}_{\mathrm{y}}^{2}}{2 \mathrm{v}_{\mathrm{j}}^{2}} \frac{\mathrm{T}_{\| \mathrm{j}}}{\mathrm{T}_{\perp \mathrm{j}}}\right)}
$$

where $\mathrm{v}_{j}$ is the thermal velocity of the $\mathrm{j}^{\text {th }}$ species. Linearizing and grouping the first-order terms gives the first-order Vlasov equation: ${ }^{91}$

$$
\begin{aligned}
& \frac{\partial f_{j}^{(1)}(\overrightarrow{\mathrm{x}}, \overrightarrow{\mathrm{v}}, t)}{\partial t}+\overrightarrow{\mathrm{v}} \cdot \frac{\partial f_{j}^{(1)}(\overrightarrow{\mathrm{x}}, \overrightarrow{\mathrm{v}}, t)}{\partial \overrightarrow{\mathrm{x}}}+\frac{e_{j}}{m_{j}}\left(\frac{\overrightarrow{\mathrm{v}} \times \overrightarrow{\mathrm{B}}_{0}}{\mathrm{c}}\right) \cdot \frac{\partial f_{j}^{(1)}(\overrightarrow{\mathrm{x}}, \overrightarrow{\mathrm{v}}, t)}{\partial \overrightarrow{\mathrm{v}}}= \\
& -\frac{e_{j}}{m_{j}}\left[\overrightarrow{\mathrm{E}}^{(1)}(\overrightarrow{\mathrm{x}}, t)+\frac{\overrightarrow{\mathrm{v}} \times \overrightarrow{\mathrm{B}}^{(1)}(\overrightarrow{\mathrm{x}}, t)}{\mathrm{c}}\right] \cdot \frac{\partial f_{j}^{(0)}(\overrightarrow{\mathrm{v}})}{\partial \overrightarrow{\mathrm{v}}}
\end{aligned}
$$


In Cartesian geometry, equation (109) can be integrated along the unperturbed orbits to obtain: ${ }^{91}$

$$
f_{j}^{(1)}(\overrightarrow{\mathrm{x}}, \overrightarrow{\mathrm{v}}, t)=-\frac{e_{j}}{m_{j}} \int_{-\infty}^{0} d \tau\left[\frac{\partial f_{j}^{(0)}(\overrightarrow{\mathrm{v}})}{\partial \overrightarrow{\mathrm{v}}}+\frac{\vec{k}}{\omega} \times\left(\overrightarrow{\mathrm{v}}^{\prime} \times \frac{\partial f_{j}^{(0)}(\overrightarrow{\mathrm{v}})}{\partial \overrightarrow{\mathrm{v}}^{\prime}}\right)\right] \cdot \overrightarrow{\mathrm{E}}^{(1)}(\overrightarrow{\mathrm{x}}, t) e^{i \mathrm{~b}_{j}(\tau, \omega)}
$$

where

$$
b_{j}(\tau, \omega)=\frac{k_{y} \mathrm{v}_{x}}{\omega_{c j}} \cos \left(\omega_{c j} \tau-1\right)+\frac{k_{y} \mathrm{v}_{y}}{\omega_{c j}} \sin \left(\omega_{c j} \tau\right)+\left(k_{z} \mathrm{v}_{z}-\omega\right) \tau
$$

and $\omega_{c j} \equiv e_{j} B_{o} / m_{j} c$ is the cyclotron frequency of the $\mathrm{j}^{\text {th }}$ species. The fields are assumed to be of the form:

$$
\begin{gathered}
\overrightarrow{\mathrm{B}}=\mathrm{B}_{0} \hat{z}, \\
\overrightarrow{\mathrm{E}}^{(1)} \propto \overrightarrow{\mathrm{E}}_{0} e^{-i(\vec{k} \cdot \vec{x}-\omega t)}, \\
\overrightarrow{\mathrm{B}}^{(1)} \propto \overrightarrow{\mathrm{B}}_{0} e^{-i(\vec{k} \cdot \vec{x}-\omega t)},
\end{gathered}
$$

and

$$
\omega=\omega_{r}+i \gamma
$$

i.e., $k$ is assumed to be real.

Typically, the dielectric tensor is calculated to determine the possible wave dispersion relations. The dielectric tensor, $\vec{K}$, is defined as

$$
-\left(4 \pi \omega i \vec{J}+\left(\omega^{2}-k^{2} c^{2}\right) \vec{E} \equiv \vec{K} \cdot \vec{E}\right) .
$$

Or, in terms of the particle flux, $\vec{\Gamma}=e \vec{J}$,

$$
-\left(4 \pi \omega i \vec{\Gamma} / e+\left(\omega^{2}-k^{2} c^{2}\right) \vec{E} \equiv \vec{K} \cdot \vec{E}\right) .
$$

However, Gary defines the dimensionless conductivity tensor, $\vec{S}_{j}(\vec{k}, \omega)$, as:

$$
\vec{\Gamma}_{j}^{(1)}(\vec{k}, \omega)=-\frac{i k^{2} c^{2}}{4 \pi e_{j} \omega} \vec{S}_{j}(\vec{k}, \omega) \cdot \vec{E}^{(1)}(\vec{k}, \omega) .
$$

and rewrites the perturbed flux, $\vec{\Gamma}_{j}^{(1)}(\vec{k}, \omega)$, in terms of the perturbed distribution function to solve for the allowed dispersion relations. For $\vec{k} \times \overrightarrow{\mathrm{B}}=0$, which applies to the firehose and ion cyclotron anisotropy instabilities, equation (110) is substituted into equation (118). After considerable algebra, equation (118) yields: 


$$
\omega^{2}-k^{2} c^{2}+k^{2} c^{2} \sum_{j} S_{j}^{ \pm}(\vec{k}, \omega)=0
$$

where

$$
S_{j}^{ \pm}(\vec{k}, \omega)=\frac{\omega_{p j}^{2}}{\left(2 \pi v_{j}^{2}\right)^{1 / 2}}\left[\zeta_{j}^{0} Z\left(\zeta_{j}^{ \pm 1}\right)-\left(\frac{T_{\perp j}}{T_{\| j}}-1\right) \frac{Z^{\prime}\left(\zeta_{j}^{ \pm 1}\right)}{2}\right]
$$

and

$$
\zeta_{j}^{m} \equiv \frac{\omega+m \omega_{c j}}{\sqrt{2}\left|k_{z}\right| \mathrm{v}_{j}}
$$

$Z(\varsigma)$ is the standard plasma dispersion function. ${ }^{90}$ Solving equation (119) for the growth rate, $\gamma$. In the limit of $\gamma<<\omega_{r}$

$$
\gamma=\frac{\pi}{2 \omega_{r}} \sum_{j} \frac{\omega_{j}^{2}}{\left(2 \pi \mathrm{v}_{\mathrm{j}}^{2}\right)^{1 / 2}}\left[-\frac{\mathrm{T}_{\perp \mathrm{j}}}{\mathrm{T}_{\| \mathrm{j}}} \frac{\omega_{r}}{k_{z}} \pm\left(1-\frac{\mathrm{T}_{\perp \mathrm{j}}}{\mathrm{T}_{\| \mathrm{j}}}\right) \frac{\Omega_{j}}{k_{z}}\right] e^{\left[\frac{\left(\omega_{r} \pm \Omega_{j}\right)^{2}}{2 k_{z}^{2} \mathrm{v}_{\mathrm{j}}^{2}}\right]} .
$$

Calculation of the growth rate of the mirror mode follows in a similar fashion for $\vec{k} \times \overrightarrow{\mathrm{B}} \neq 0.91$

Choosing a value for the growth rate establishes a constraint on the values of the ion anisotropy, $\mathrm{T}_{\perp} / \mathrm{T}_{\|}$, and the values of parallel beta, through the plasma frequency (density) and the anisotropy (parallel temperature). For a fixed value of growth rate, one can determine the ion temperature anisotropy, plasma frequency and cyclotron frequency, for all of the allowed $k$ 's and $\omega$ 's.

Of the three possible instabilities, only the firehose instability requires $T_{\|}>T_{\perp}$ and high $\beta$. As shown by Kennel and Petschek, ${ }^{87}$ the firehose instability has a maximum growth rate for $\vec{k} \times \overrightarrow{\mathrm{B}}=0$, is right-hand circularly polarized, and evolves out of the magnetosonic/whistler wave as the ion temperature anisotropy decreases. The wave frequency is roughly equal to the ion gyrofrequency. The predicted growth rates from equation (122), and the real frequencies, from equation (119), are shown in Figure 42 for three different anisotropy values. Equation (122) is consistent with the Kennel and Petschek calculation that takes the low frequency limit of a right-hand, circularly polarized electromagnetic wave propagating parallel to the background magnetic field to determine the wavelengths and frequencies of the most unstable wave. 

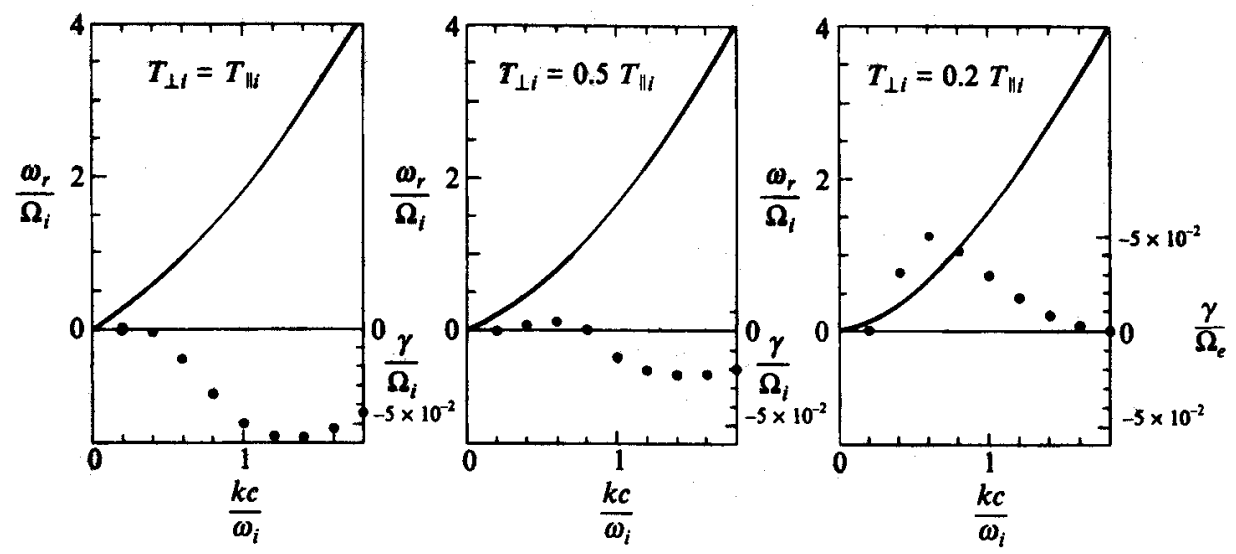

Figure 42: The real frequency (solid line) and growth rates (dots) for the ion firehose instability for three different ion temperature anisotropies. Both the real frequency $\left(\omega_{r}\right)$ and the growth rate $(\gamma)$ are normalized with respect to the ion cyclotron frequency $\left(\Omega_{i}\right)$. The plasma parameters used in the calculation were $T_{e}=T_{p}, \mathrm{v}_{\text {alfven }} / c=10^{-4}$, and $\beta=2$.

Although the ion cyclotron anisotropy and the mirror instabilities occur over roughly the same values of ion beta, they are distinctly different. The ion cyclotron anisotropy instability has a lower anisotropy threshold than the mirror instability for low values of beta $(\beta<6) .{ }^{92}$ In a stationary, homogenous plasma, the mirror mode has zero real frequency, which means it is a DC signal. The ion cyclotron anisotropy instability occurs at frequencies lower then the ion cyclotron frequency, usually in the range of $\left(0.2 \omega_{c i}-0.8 \omega_{c i}\right)$. At low values of beta, the ion cyclotron anisotropy instability is predicted to dominate over the mirror instability. However, simulations have shown that a small helium-ion component can reduce the linear growth rate of the proton cyclotron instability without significantly affecting the mirror instability. ${ }^{93}$ Future experiments in LEIA using two different ion species could be performed to observe the changes in the ion cyclotron anisotropy instability as a function of heavier ion concentration. At high values of $\beta(\beta>6)$ the mirror mode has a lower anisotropy threshold. ${ }^{92}$

The ion cyclotron anisotropy instability is characterized by primarily transverse fluctuations, while the mirror fluctuations are compressional for $\beta<1$. The growth rates 
for three different values of ion temperature anisotropies for the ion cyclotron anisotropy instability are shown in Figure 43. The growth rates of the mirror instability for three ion temperature anisotropies are shown in Figure 44. The results shown in Figure 43 can be compared to the calculations by Davidson and $\operatorname{Ogden}^{94}$ for similar parameters (Figure 45). Davidson and Ogden solved the dispersion relation in the limit of $T_{i \|}=0$.

$$
-c^{2} k^{2} \mp \omega_{p e}^{2} \frac{\omega}{\omega_{c e}}-\omega_{p i}^{2} \frac{\omega}{\omega \pm \omega_{c i}}-\frac{1}{2} \beta_{i \perp}^{2} c^{2} k_{z}^{2} \frac{\omega_{c i}^{2}}{\left(\omega \pm \omega_{c i}\right)^{2}}=0 .
$$

In the $T_{i \|}=0$ limit, the $T_{i \|}$ terms cancel in the dispersion relation. As seen in Figure 43, the parallel wavenumber of the fastest growing mode increases with increasing ansotropy, consistent with equation (123).

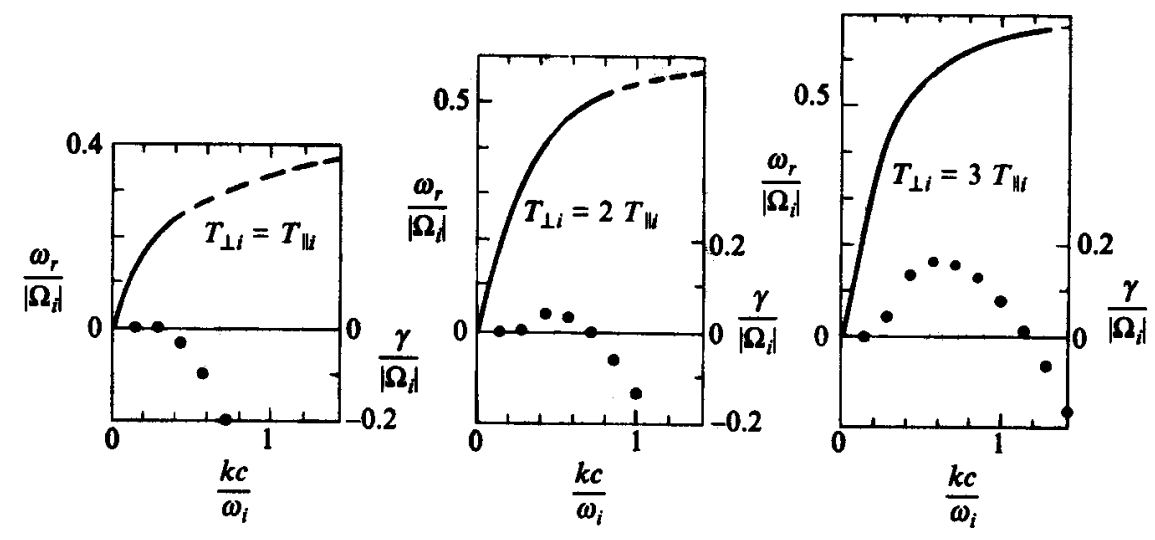

Figure 43: The real frequency (solid line) and growth rate (dots) for the ion-cyclotron instability for three different ion temperature anisotropies. $\beta=1 .{ }^{91}$ 


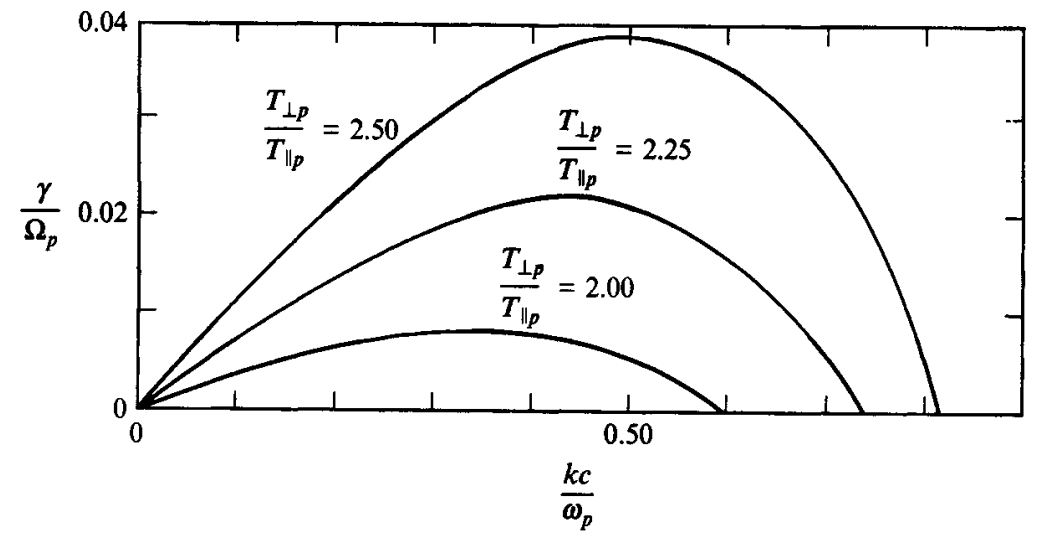

Figure 44: Growth rates for the mirror instability for different ion temperature anisotropies. $\beta=1 .{ }^{91}$

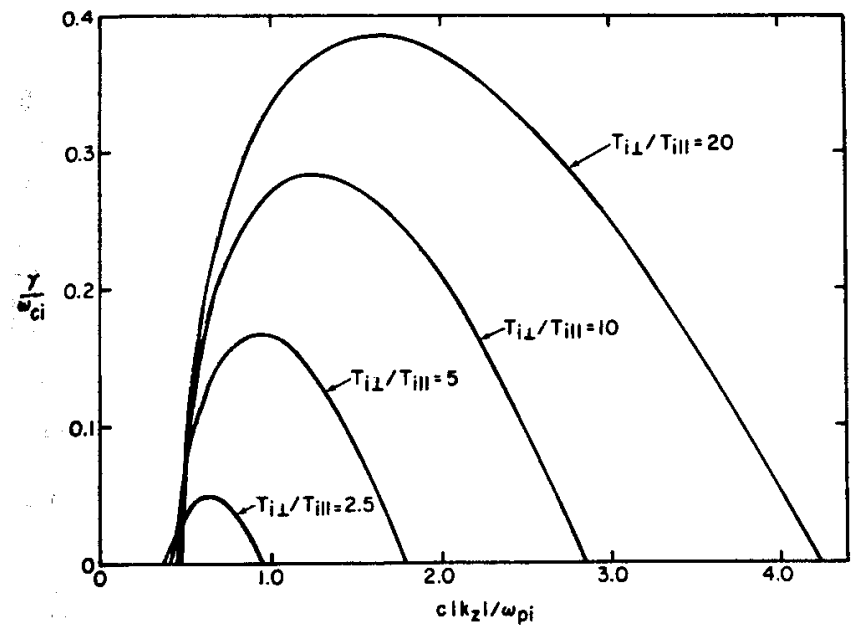

Figure 45: Growth rates for the ion cyclotron instability for four different values of ion temperature anisotropy. ${ }^{94}$ These results are consistent with those found the methods of Gary (Figure 43).

For fixed growth rates, it is possible to use equation (122), the growth rate equation, to generate curves of $\beta$ versus $T_{i \perp} / T_{i \|}$ for the different instabilities. Families of such curves are shown in Figure 46. Note that the firehose instability occurs only for anisotropies less than one and at large $(\beta \sim 1)$ values of beta. The ion cyclotron anisotropy instability occurs over a wide range of ion beta values and generally requires an anisotropy of less than five. 


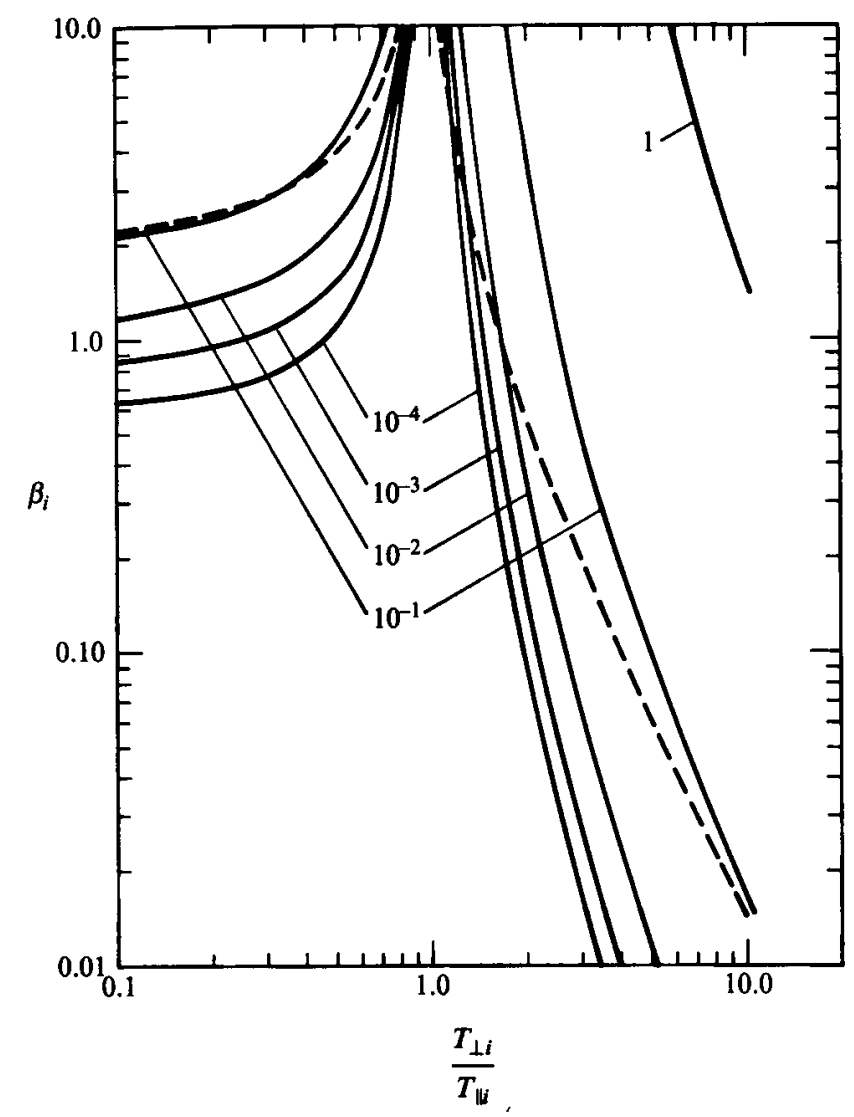

Figure 46: Beta versus Anisotropy inverse correlation for the three different instabilities for different growth rates. The solid lines to the left of an anisotropy of unity correspond to the ion firehose instability. The solid lines to the right of an anisotropy of unity correspond to the ion cyclotron instability. The dashed lines correspond to the MHD thresholds for the non-resonant firehose and mirror instabilities. ${ }^{91}$

In addition to analytic investigations, both the mirror and ion cyclotron anisotropy instabilities have been the subject of numerous computational studies. Most of the studies have used the 1-D hybrid code developed by Winske and Omidi. ${ }^{95}$ A hybrid code treats the electrons as a massless fluid and treats the ions as discrete marcroparticles. This treatment allows calculations to be done on the ion time scale instead of the electron time scale, thereby focusing on the ion physics exclusively. The computations include all three components of the ion velocities, the currents and the electric and magnetic fields. To model bi-Maxwellian effects in a 1-D code, two ion components are used: a cold ion background and a beam drifting with respect to the cold component along the background 
magnetic field. All of the calculations are performed in the rest frame of the electrons, where the ion current is zero. In the one-dimensional calculations, the $x$ coordinate is the direction of $k$. In the two-dimensional codes, the magnetic field is along the $x$-coordinate and the waves develop in the $x-y$ plane. Other researchers have studied the expected wave spectra using 2-D hybrid codes. ${ }^{96}$

Recently, driven simulations have been reported in literature. ${ }^{97}$ In driven simulations, the ions' velocities are periodically altered to increase the ion temperature anisotropy. This method is done to simulate a driving mechanism that keeps the ion's anisotropy near the value needed to excite the EMITA instability, similar to the conditions believed to exist in the magnetosheath. Many of the spacecraft observations occur in a region of space called the plasma depletion layer (PDL). Plasma constantly enters the PDL from the magnetosheath proper with a higher ion temperature anisotropy than in the PDL. Even though the anisotropy begins to decrease in the PDL, there is a constant supply of ions with large enough anisotropies to maintain a large, steady, ion temperature anisotropy.

Besides predicting the growth rates and wavenumbers for the various instabilities, Gary also noticed that a fixed value of the maximum growth rate of the ion cyclotron anisotropy instability leads to an inverse correlation between the ion temperature anisotropy and the ion parallel beta. The correlation is of the form

$$
\frac{T_{\perp}}{T_{\|}}-1=\frac{S}{\beta_{\|}^{\alpha}},
$$

where $S$ is dimensionless and depends on the choice of maximum growth rate and the density of the hot protons and $\alpha \approx 0.5$. Fit parameters based on equation (124) for various theoretical studies are tabulated in Table 6 for the ion temperature instability.

The choice of a particular value of maximum growth rate is predicated on the assumption that once such a value of growth rate is reached, the amplitude of the instability becomes large enough to induce strong velocity-space diffusion and thereby supresses the ion temperature anisotropy, i.e., a self-limiting process as described by Manheimer and Boris. ${ }^{1}$ Note that it is the maximum growth rate, regardless of the particular $\omega$ and $k$ of the instability, that is held fixed. As the ion temperature anisotropy and parallel ion beta change, the wavenumber and frequency associated with the 
maximum growth rate also change. However, the assumption here is that when the growth rate of the instability reaches some maximum value, the growth rate can increase no further.

For values of ion temperature anisotropy and parallel ion beta that lie below the maximum growth rate curve, fluctuations driven by the anisotropy can exist and grow in amplitude, but at a rate less than the maximum growth rate. As the value of beta increases, very little anisotropy is required to reach the maximum growth rate. Thus, at high values of beta, only modest values of ion temperature anisotropy can exist. If, at a fixed value of beta, the value of anisotropy were increased, the growth rate of the instability would increase until the maximum value was reached. At that point, equilibrium between fluctuation growth rate and velocity space diffusion would be reached and the anisotropy could not be increased any further. When collisions are considered, there is a little change in the above picture. "It is expected that collisions will result in fewer anisotropy measurements near the maximum growth rate curve. Relatively more anisotropy measurements will lie below the maximum growth rate curve, representing cases in which collisions have had time to further the anisotropy." 98 The approach used in Gary's calculations is linear and therefore the maximum growth rate calculations are based purely on linear theory. The interpretation of the balance between the wave's growth due to finite anisotropy and the scattering in velocity-space due to the fluctuation amplitude resembles a quasi-linear interpretation.

Table 6: Fit parameters for the ion temperature anisotropy - beta inverse correlation for different simulations and growth rates in the linear Vlasov model.

\begin{tabular}{|c|c|c|}
\hline Gary Model & $\boldsymbol{S}$ & $\alpha$ \\
\hline 1-D Hybrid: Driven Case & 0.55 & 0.52 \\
\hline Linear Vlasov - $\left(\gamma=10^{-4} \Omega_{p}\right)^{97}$ & 0.35 & 0.42 \\
\hline Linear Vlasov - $\left(\gamma=10^{-3} \Omega_{p}\right)^{97}$ & 0.43 & 0.42 \\
\hline Linear Vlasov - $\left(\gamma=10^{-2} \Omega_{p}\right)^{97}$ & 0.65 & 0.40 \\
\hline Linear Vlasov - Ion Cyclotron $^{99}$ & 0.66 & 0.40 \\
\hline Linear Vlasov - Mirror $^{99}$ & 0.97 & 0.61 \\
\hline
\end{tabular}




\subsection{Recent Spacecraft Observations}

Researchers using data from the Active Magnetosphere Particle Tracer Explorers (AMPTE) Charge Composition Explorer (CCE) spacecraft, the APMTE Ion Release Module (IRM), and the WIND spacecraft have all reported the existence of an upper bound on the ion temperature anisotropy versus ion beta in the Earth's magnetosheath. Wave activity around the cyclotron frequency was often, but not always observed in conjunction with large ion temperature anisotropies.

Though they were part of the same mission, there are significant differences between the CCE and IRM spacecraft. The apogee of AMPTE/CCE was $8.8 R_{E}$ (Earth radii) and the apogee of IRM was $18.8 R_{E}$. Each spacecraft was equipped with magnetometers to measure the three dimensional magnetic field fluctuations. AMPTE/IRM measured the energy distribution of ions with a three-dimensional (spin and azimuthal) electrostatic analyzer. However, the AMPTE/CCE plasma instrument was a simple two-dimensional electrostatic analyzer (spin only). The AMPTE/CCE ion temperature anisotropy measurements were sensitive to variations in the direction of the magnetic field as the instrument lacked resolution out of the spin plane of the spacecraft. ${ }^{100}$ Thus, it is likely that the AMPTE/CCE ion temperature measurements over-estimate the level of ion temperature anisotropy in the magnetosheath. ${ }^{101}$

The AMPTE spacecraft data relevant to this investigation were taken in the magnetosheath and across the magnetopause (see Figure 47). Near the magnetopause, magnetosheath flow can give rise to a region of decreased plasma density and increased

magnetic field strength, ${ }^{4}$ i.e. lower beta. This region is called a plasma depletion layer (PDL). When a PDL is formed, the plasma and field in it are representative of the magnetopause and not the magnetosheath. Since the PDL typically has a lower beta than the magnetosheath, the mirror instability is not as likely to be present in the PDL and care must be used in comparing PDL and magnetosheath measurements. ${ }^{102}$ 


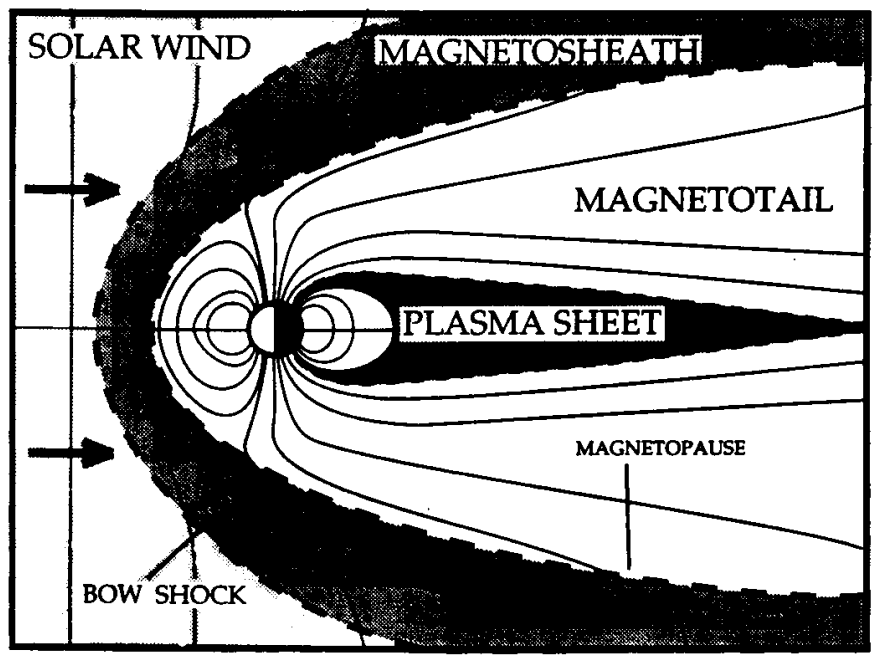

Figure 47: Location of magnetosheath and magnetopause. The PDL occurs on the sunward side of the magnetopause. ${ }^{91}$

Anderson et al. reported a study of ion temperature anisotropies in the magnetosheath and the plasma depletion layer using AMPTE/CCE data. ${ }^{103}$ They limited their study only to events where magnetic fluctuations were also observed. Data from Anderson et al. ${ }^{103}$ are shown in Figure 48. The data are grouped by the local plasma environment. In high $\beta$ - low $A$ environments, where $A \equiv T_{\perp} / T_{\|}-1$, the fluctuations are compressional, while in low $\beta$ - high $A$ environments the waves are transverse. In each case, a least-squares fit of the form $A=1-S / \beta^{\alpha}$ is performed for the ion anisotropy versus parallel ion beta data. 


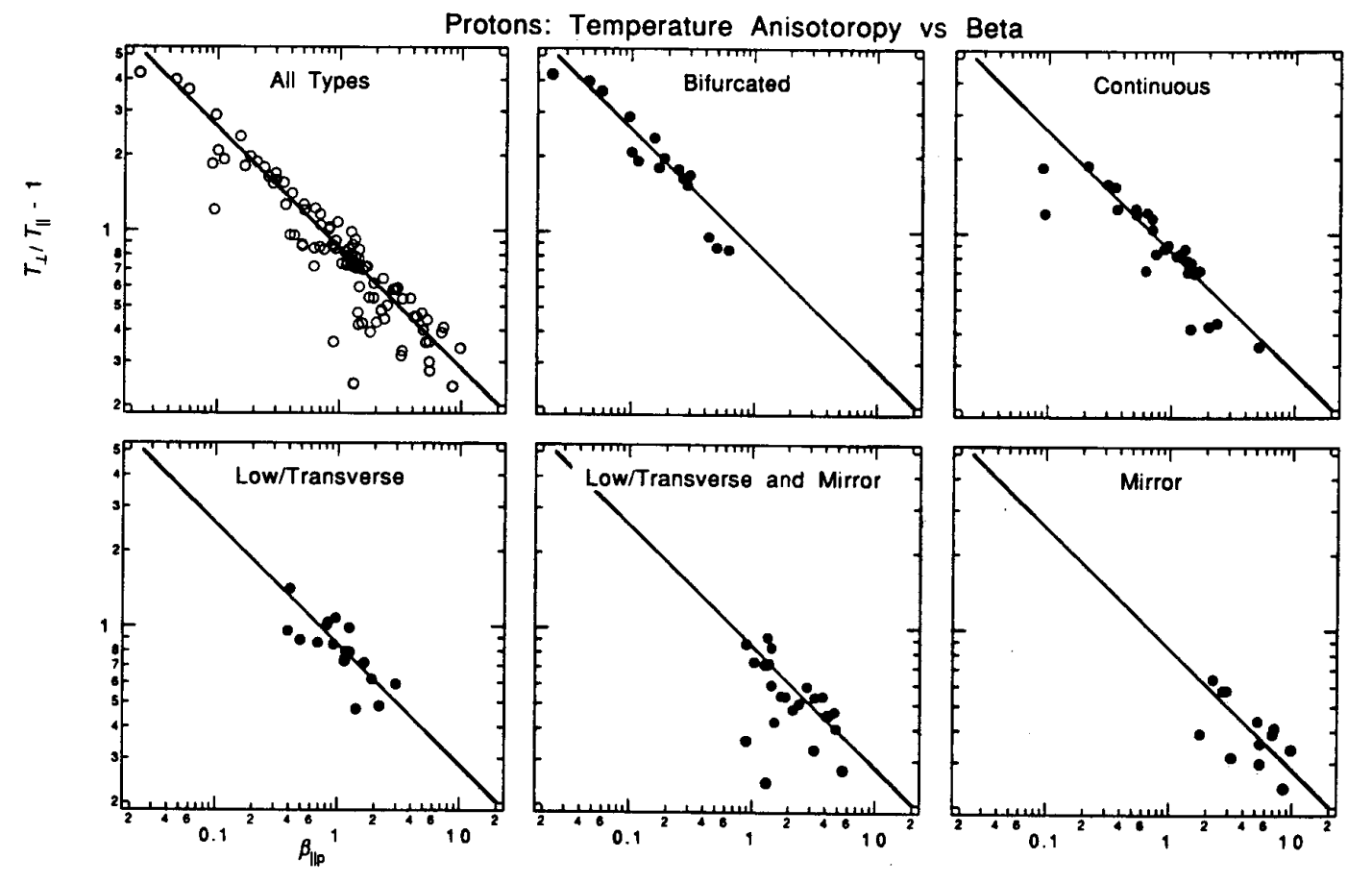

Figure 48: Anderson's data from the AMPTE/CCE spacecraft. Bifurcated data are correlated with observations of transverse waves with two broad maxima in the frequency spectra. Data classified as continuous had transverse waves with continuous power spectra extending above and below half the proton cyclotron frequency. Low/Transverse data consisted of data with transverse waves below half the proton cyclotron frequency.

Data classified as low/transverse and mirror consists of intervals with a prominent compressional peak and a transverse peak, below half the proton cyclotron frequency, at different frequencies. Mirror data is dominated by compressional perturbations with no discernible transverse peak. ${ }^{103}$

The AMPTE/CCE data indicate that during periods of low frequency wave activity, there is an inverse correlation between the upper bound on the temperature anisotropy of the ions and the parallel ion beta. The frequency spectra of the waves were also compared to the predictions of linear Vlasov theory. The observed transverse wave spectra were in good agreement with the theoretical predictions for the ion cyclotron wave, while the observed compressional spectral structures were in good agreement with the predictions for the mirror mode. ${ }^{103}$ The mirror mode-like waves were observed when the spacecraft was in the magnetosheath proper (high $\beta$ ), while the ion cyclotron-like waves were observed when the spacecraft was in the PDL (low $\beta$ ). 
Using data from the AMPTE/IRM spacecraft, Phan et al. obtained similar results. ${ }^{104}$ Phan et al. did not study the magnetic fluctuations in detail, but assumed they were mirror waves. ${ }^{104}$ Figure 49 shows the anisotropy versus parallel ion beta data reported from the AMPTE/IRM spacecraft. ${ }^{104}$

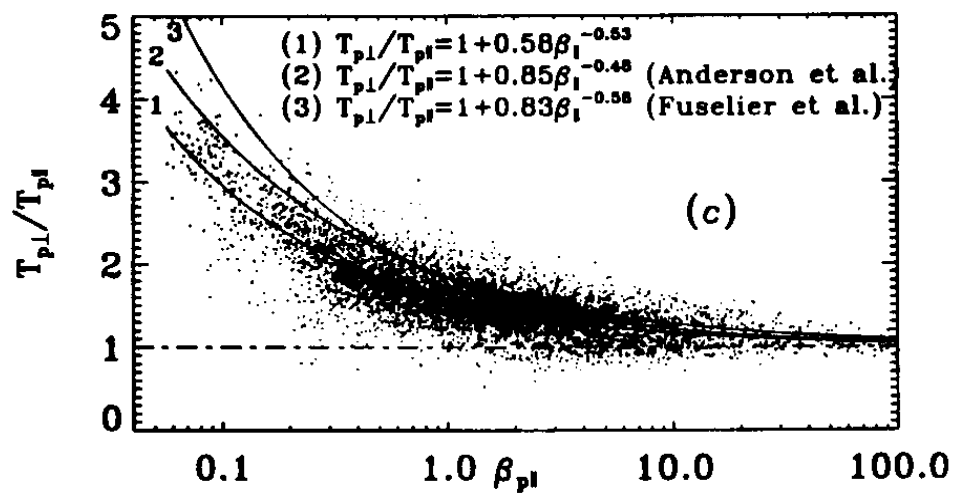

Figure 49: Magnetosheath proton temperature anisotropy versus parallel proton beta data from the AMPTE/IRM spacecraft. ${ }^{104}$

Also shown in Figure 49, are the anisotropy-beta inverse correlations reported by Anderson et $a l .{ }^{4}$ and by Fuselier et al.. ${ }^{6}$ Phan et al. also performed a study of ion temperature anisotropy limits in the magnetosphere using WIND data. ${ }^{105}$ Parameters for fits to the anisotropy-inverse beta correlation for the various spacecraft data sets are listed in Table 7. Again, even though magnetic fluctuations were observed, Phan et al. did not discuss the characteristics of the magnetic fluctuations for the WIND observation.

Although Figure 48 and Figure 49 demonstrate that the inverse-beta correlation exists during periods of wave activity with distinct low frequency peaks, Fuselier ${ }^{106}$ has shown that data from the AMPTE/CCE spacecraft with broadband wave activity also exhibit an inverse correlation of anisotropy and beta. Figure 50 and Figure 51 show typical magnetic spectra for a case of distinct low frequency peaks and a case of broadband waves, respectively. In Figure 52, $\mathrm{T}_{\perp} / \mathrm{T}_{\|}$versus $\beta_{i \|}$ data corresponding to periods of broadband magnetic fluctuations are plotted along with $\mathrm{T}_{\perp} / \mathrm{T}_{\|}$versus $\beta_{i \|}$ data corresponding to periods of wave activity with distinct peaks in the low frequency power spectra. The data corresponding to broadband wave activity was observed to have, on average, higher betas and lower anisotropies than the data corresponding to periods with 
clear power spectrum peaks. The anisotropy data corresponding broadband wave activity is consistent with the AMPTE/CCE data. A fit to anisotropies for only the broadband data yields:

$$
\mathrm{T}_{\perp} / \mathrm{T}_{\|}=1+0.83 \beta_{\|}^{-0.58}
$$

Table 7: Different values of $S$ and $\alpha$ for different authors.

\begin{tabular}{|c|c|c|}
\hline Author & $S$ & $\alpha$ \\
\hline Anderson - AMPTE/CCE ${ }^{103}$ & 0.85 & 0.48 \\
\hline Fuselier - AMPTE/CCE ${ }^{106}$ & 0.83 & 0.58 \\
\hline Phan - AMPTE/IRM ${ }^{104}$ & 0.58 & 0.43 \\
\hline Phan - WIND $^{105}$ & 0.56 & 0.49 \\
\hline
\end{tabular}

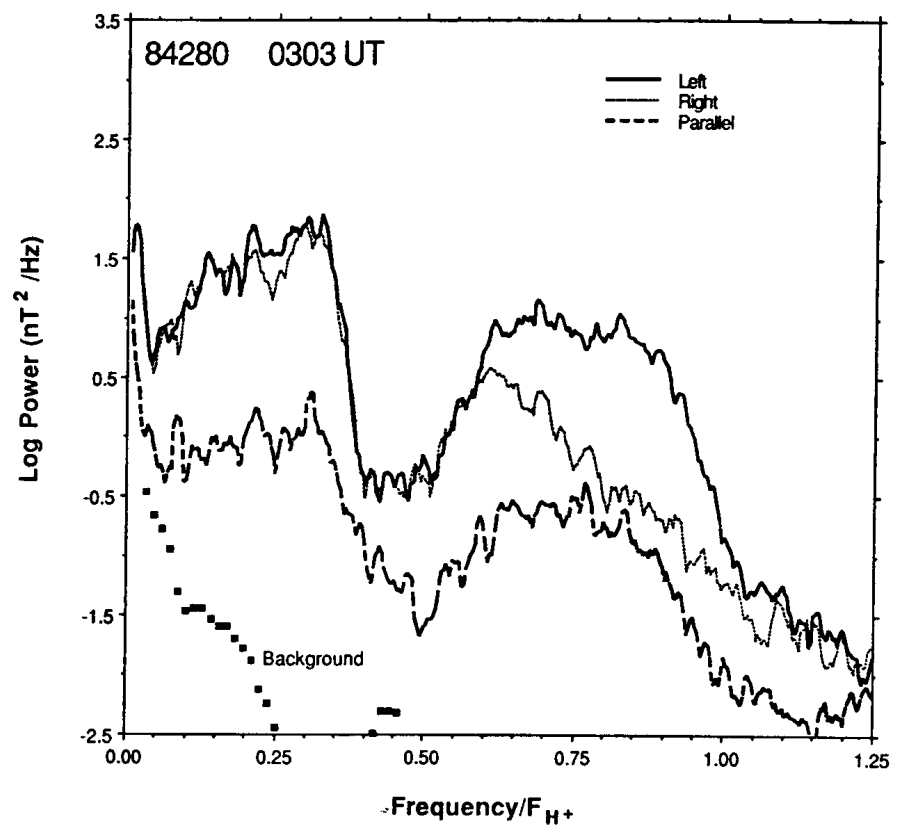

Figure 50: Magnetic power spectrum from the plasma depletion layer showing distinct low frequency peaks. This is a typical spectrum from AMPTE/CCE. The lower frequency is identified as the helium ion cyclotron wave, while the higher peak is identified as the proton cyclotron wave. ${ }^{106}$ 


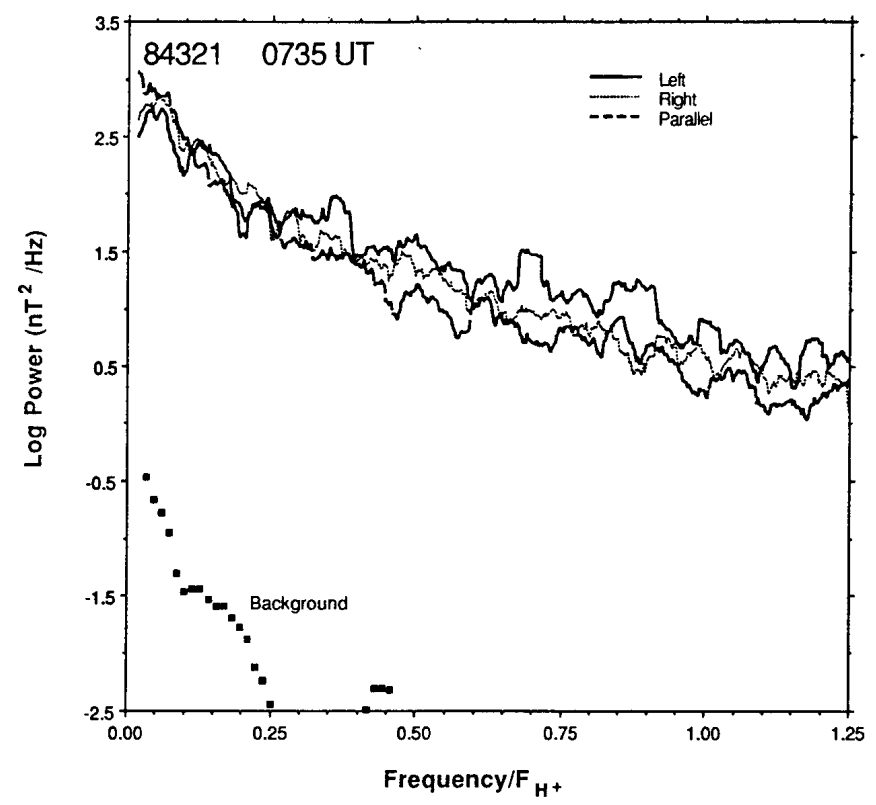

Figure 51: Magnetic power spectrum from the quasi-parallel magnetosheath. There is broadband wave activity, but no distinct low frequency peaks. ${ }^{106}$

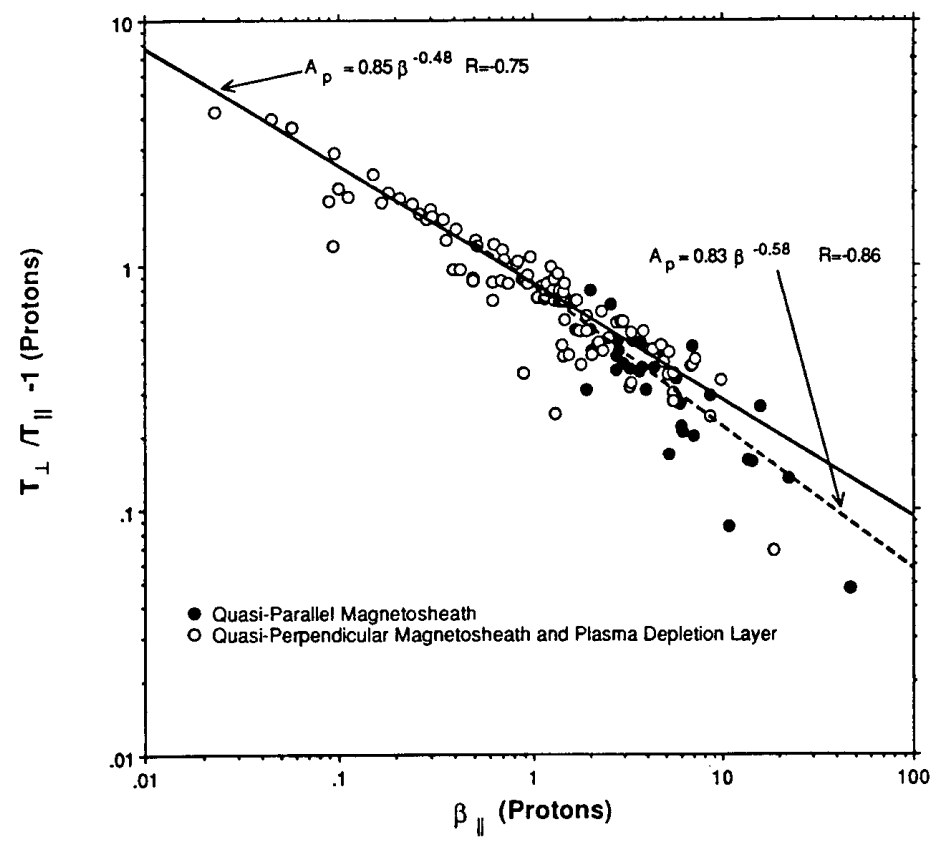

Figure 52: Anisotropy versus beta data for broadband wave activity (solid circles) and clear low frequency activity (open circles). Note the broadband activity corresponds to higer beta and lower anisotropies because the entire data set was obtained from the magnetosheath. ${ }^{106}$ 


\subsection{Previous Experimental Investigations of EMITA Instabilities}

Alfvén ion cyclotron instabilities (AIC), or ion cyclotron anisotropy instabilities, have been observed before in the laboratory. Experiments in both the tandem mirror machine, GAMMA $10^{107}$ and the Tandem Mirror experiment (TMX) ${ }^{108}$ observed AIC waves along with $T_{\perp}>T_{\|}$anisotropies. Casper and Smith presented the first experimental evidence for the presence of the Alfvén ion cyclotron (AIC) instability through measurements of magnetic fluctuations just below the hydrogen cyclotron frequency and indirect measurements of the temperature anisotropy. ${ }^{108}$ Their values of $\beta_{i \perp}$ and $\beta_{i \perp} \mathrm{A}^{2}$ exceeded the Davidson and Ogden theoretical threshold $\left(\beta_{i \perp} \mathrm{A}^{2} \geq 3.5\right)$ for the AIC instability. ${ }^{109}$ Here $A=T_{\perp} / T_{\|}$. Thus, they concluded the observed transverse right circularly polarized waves must be AIC waves. Similar results were also obtained from GAMMA 10, ${ }^{110,111}$ though their values of $\beta_{i \perp} \mathrm{A}^{2}$ were much lower than the theoretical threshold $\left(\beta_{i \perp} \mathrm{A}^{2} \geq 0.3\right)$ (Figure 53). The most compelling aspect of those experiments was the observation that the AIC waves only appeared above a $\beta_{i \perp} \mathrm{A}^{2}$ threshold. However, there was no indication of an upper bound on the ion temperature anisotropy even though relatively large betas were achieved. Figure 54 compares the $\beta_{i \perp} \mathrm{A}^{2}$ threshold observed by the GAMMA 10 group to the space data and theory curves discussed previously. A key difference between the GAMMA 10 results and the spacecraft measurements is the range of $\beta_{i \perp}$. Although large values of $\beta_{i \perp}$ were obtained, $\beta_{i \|}$ may not have been large enough to keep the plasma near the instability threshold seen in space and in theory. 


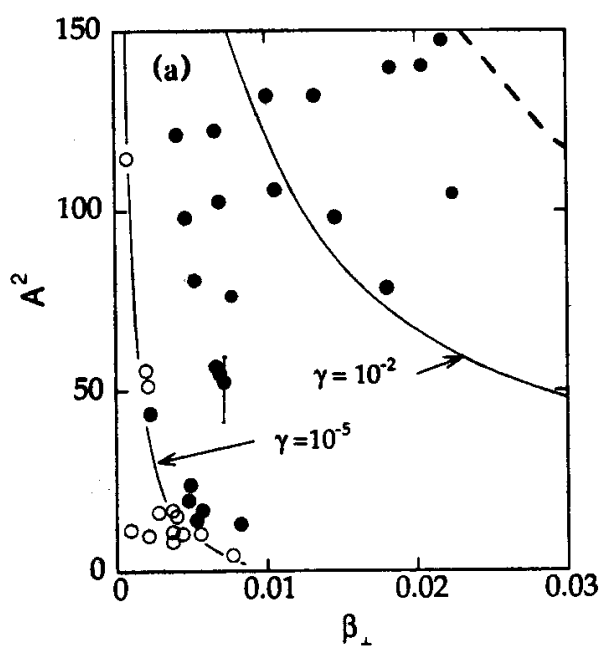

Figure 53: Results from the GAMMA 10 experiment. ${ }^{110}$ The dashed line is the theoretical curve for $\left(\beta_{\perp} \mathrm{A}^{2} \geq 3.5\right)$. The solid curves are for different growth rates for the AIC instability. The solid circles represent measurements when wave activity was present and the open circles when wave activity was not present. There is no apparent upper bound on the anisotropy as there is in the spacecraft data. ${ }^{110}$

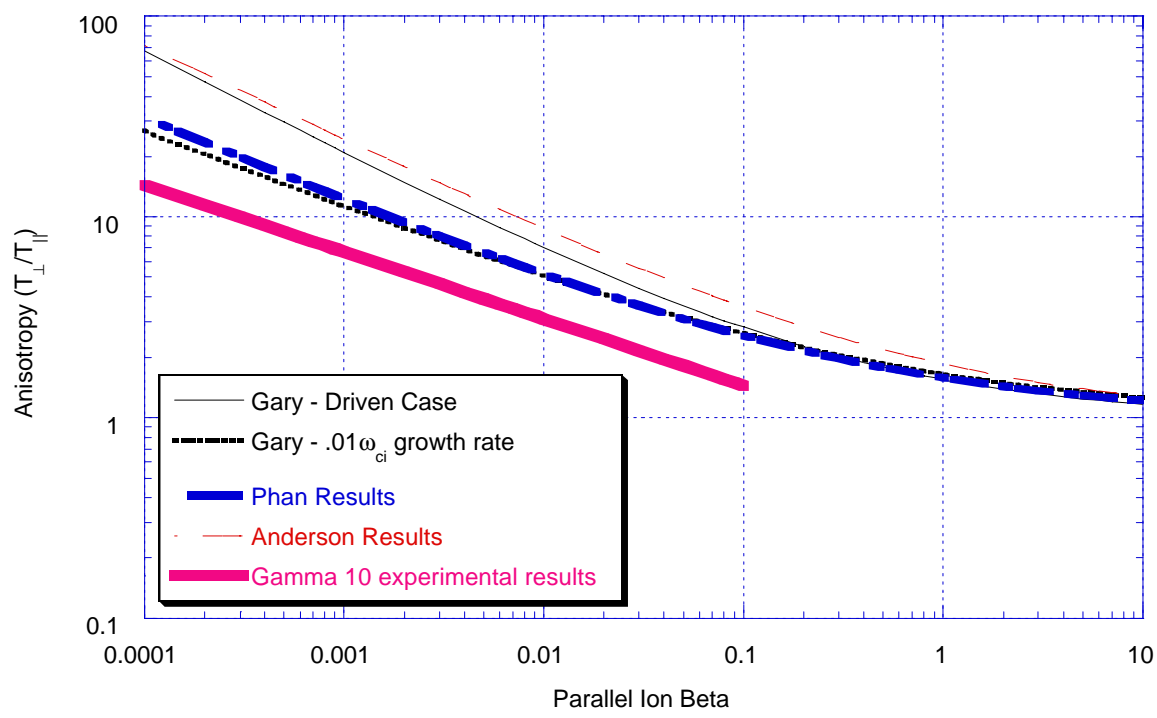

Figure 54: The GAMMA 10 observed instability threshold compared to other computation models and spacecraft results. 


\section{EXPERIMENTAL RESUltS ON ION TEMPERATURE ANISOTROPY UPPER BOUND}

As part of this investigation into EMITA instabilities, two different experiments were performed. The first was designed to determine if an inverse correlation between the ion temperature anisotropy and the parallel ion beta exists in LEIA plasmas. The second was designed to determine if ion temperature anisotropy reduction could be directly associated with low frequency electromagnetic waves. In section 5.1, all of the LEIA measurements are compared to the theory calculations and space observations. Section 5.2 examines the role collisions play in limiting the ion temperature anisotropy. Section 5.3 reviews evidence concerning the role low frequency electromagnetic waves play in the reduction of anisotropy between two axial positions in LEIA. The collisionality of the laboratory plasma is a significant effect and is one of the limitations of laboratory modeling of space plasmas. Magnetosheath plasmas are collisionless $\left(\lambda_{m f p} / L>>1\right)$ while the experiment is marginally collisional $\left(\lambda_{m f p} / L \sim 1\right): \lambda_{m f p}$ is the ion mean free path and $L$ is the system scale size. The experiments also have a boundary (the vacuum chamber), which is not present in space, and use a heavier ion species, argon instead of hydrogen. In addition, the experiment only uses one species of ions instead of two, which is commonly observed in the magnetosheath (hydrogen and helium). Finally, the ratio of the electron temperature to the ion temperature is much higher in the experiment $(5-10)$ than in the simulations $^{91}$ or in the magnetosheath. ${ }^{4}$

\subsection{Inverse Correlations of $T_{i \perp} / T_{i \|}$ and $\beta_{i \|}$}

Previous experiments demonstrated that an intrinsic ion temperature anisotropy exists in HELIX. ${ }^{112}$ The anisotropy persists as the plasma expands from HELIX into LEIA and supplies an initial anisotropy that can drive instabilities in LEIA. This situation is analogous to the driven case that has been studied computationally and in the in-situ PDL data.

Ion temperature anisotropies in LEIA were measured over a wide range of plasma conditions. Every experiment was performed using argon gas at a HELIX fill pressure of 
1.8 mTorr. The HELIX magnetic field ranged from $399 \mathrm{G}$ to $1264 \mathrm{G}$ and the LEIA magnetic field ranged from $18 \mathrm{G}$ to $70 \mathrm{G}$. An $8 \mathrm{MHz}$ rf driving signal, at a power of $400 \mathrm{~W}$, was used to drive a $19 \mathrm{~cm}$ right helical antenna to generate the plasma in HELIX. For each experimental configuration, both the ion temperature anisotropy and the plasma beta were measured. The ion temperatures were measured both upstream (re-entrant probe) and downstream (radial probe). The beta was calculated based on Langmuir probe density measurements at both axial positions and the vacuum magnetic field. Plotted on a $\log -\log$ scale in Figure 55 and a linear scale in Figure 56 are the average anisotropies versus average parallel ion beta. The averaged quantities were calculated from the average of a series of ion temperature measurements for a given experimental configuration. To estimate the error in the average measurements, "super-averaged" data values were also calculated. The "super-averaged" data are obtained by averaging the average anisotropies and average betas for similar experimental configurations used on different days. The "super-averaged" data are plotted with averaged data, along with the standard deviations from each "super averaged" data set. The deviations of the ion temperature anisotropy and parallel ion beta were typically $10-20 \%$. Figure 55 and Figure 56 include both upstream and downstream measurements. The data stretches over roughly two orders of magnitude in beta and one order of magnitude in ion temperature anisotropy. The data exhibit a clear upper bound on the anisotropy level that is in good agreement with both extrapolation of the AMPTE/IRM results and simulations of the ion cyclotron anisotropy instability. The data best fit the ion cyclotron anisotropy instability simulations that used $\gamma=10^{-3} \omega_{c i}$. Therefore, this simulation result was chosen to compare to the data. In order to compare the data to the spacecraft measurements, the fits to the spacecraft data were extrapolated to include the range of betas spanned in the experiment. Low frequency fluctuations are present in nearly all of the data. 


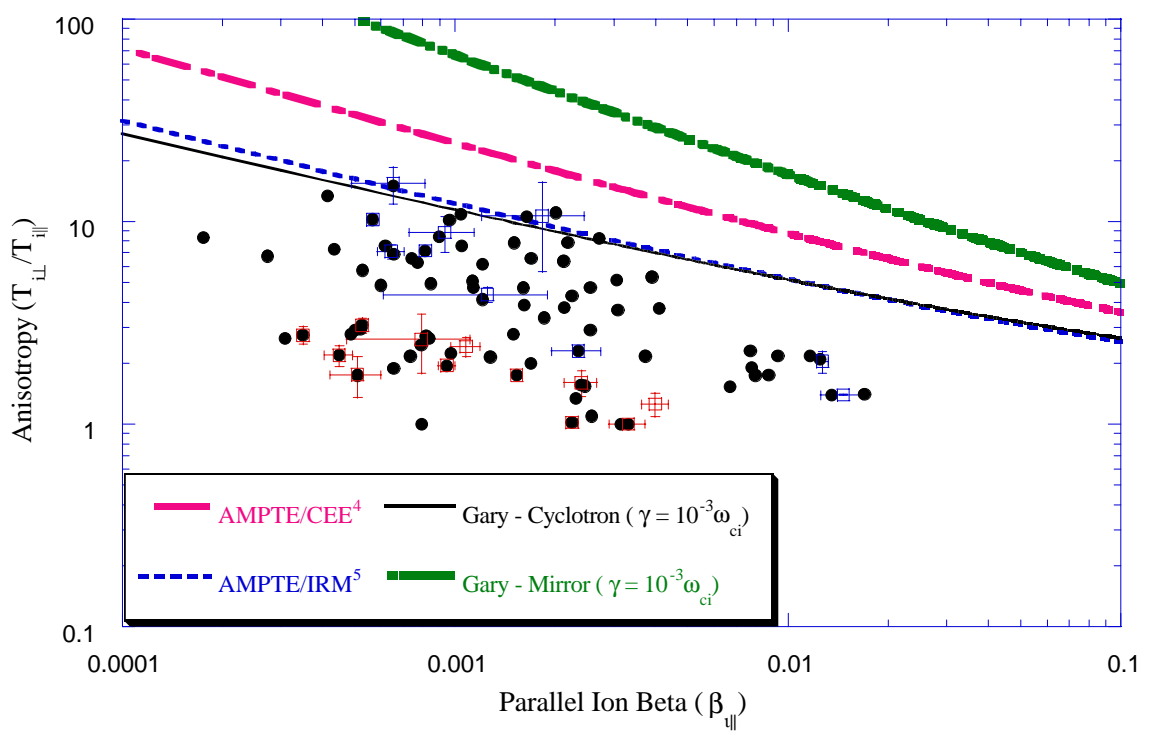

Figure 55: Ion temperature anisotropy versus parallel ion beta for all data. Solid dots are the averaged data and the open squares are the super-averaged data. Also shown are the extrapolated curves for the AMPTE/CCE data, AMPTE/IRM data and thresholds for the mirror and ion cyclotron anisotropy instability from linear Vlasov theory. 


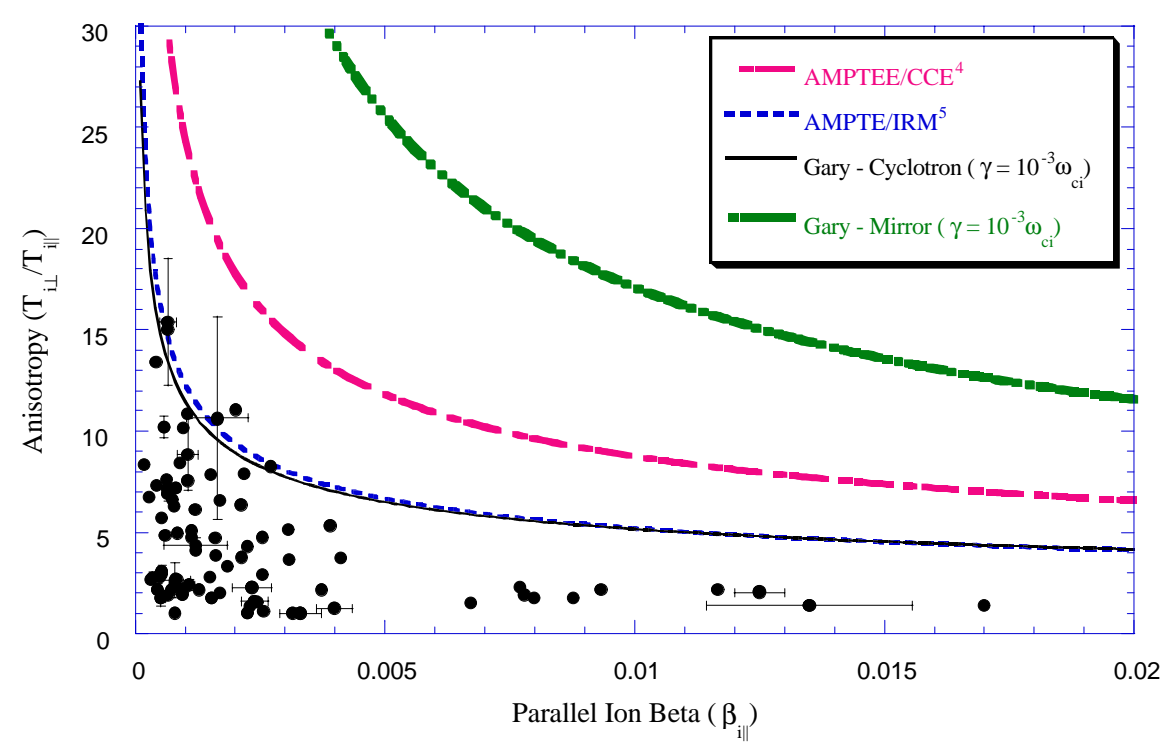

Figure 56: Same data as Figure 55 plotted on linear axes. Solid dots are the averaged data and the open squares are the super-averaged data. Also shown are the curves for the extrapolated AMPTE/CCE data, AMPTE/IRM data and simulations of the mirror and ion cyclotron anisotropy instability from linear Vlasov theory.

Upstream data (closer to HELIX) only and downstream data (farther from HELIX) only are plotted in Figure 57 and Figure 58 respectively. The upstream data is examined in detail because it spans a larger range of beta values than the downstream data. The upper bound of the upstream data is consistent with both the theoretical predictions for a choice of $\gamma=10^{-3} \omega_{c i}$ and the AMPTE/IRM spacecraft observations. A fit to the upstream data with the scaling relationship developed by Gary et al., equation (124), yields values of .38 for $S$ and .39 for $\alpha$. The value obtained for $S$ is considerably smaller than that determined by fits to the space data and undriven simulation results. Note that in the driven simulations, the values for $S$ were smaller than in the undriven cases. 


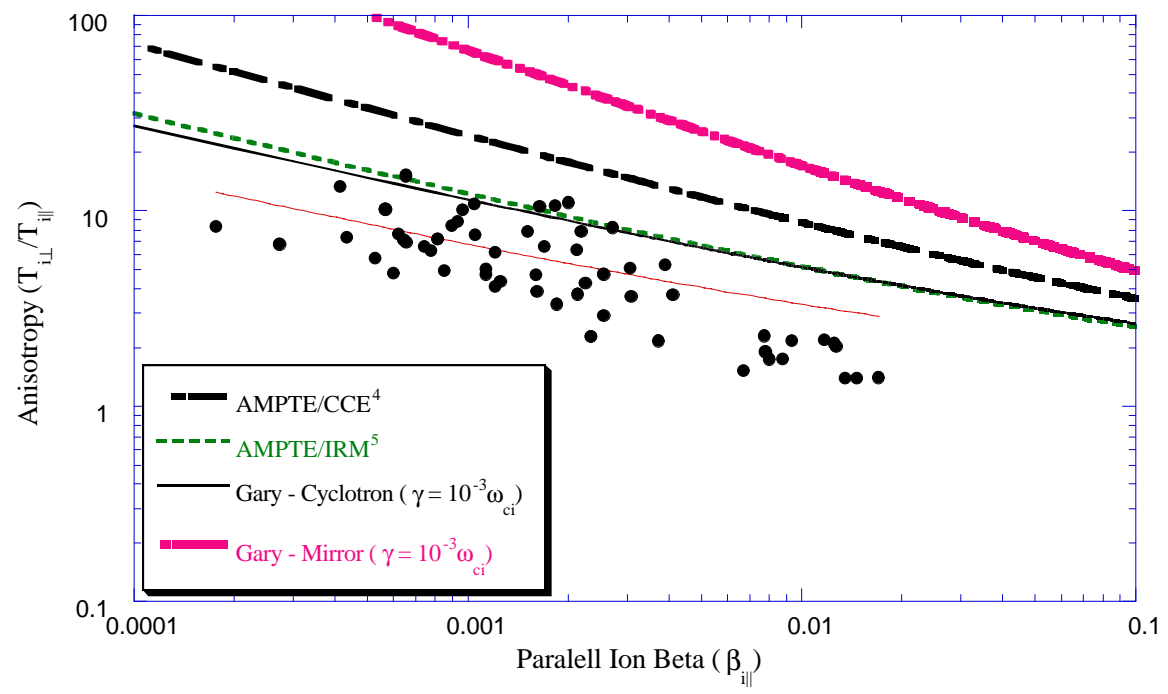

Figure 57: Ion temperature anisotropy versus parallel ion beta for upstream data points only. The data are fit by the equation $T_{\perp} / T_{\|}-1=S \beta_{i \|}^{-\alpha}$ for $S=0.38$ and $\alpha=0.39$.

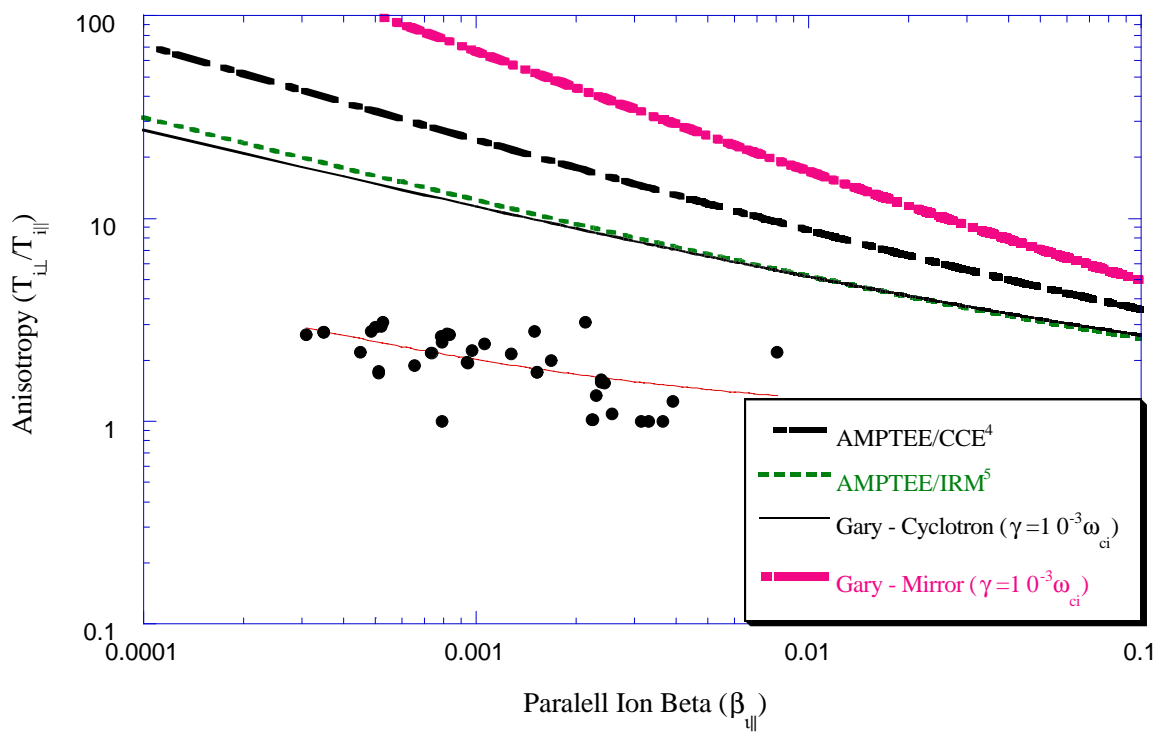

Figure 58: Ion temperature anisotropy versus parallel ion beta for downstream data only. The data are fit by the equation $T_{\perp} / T_{\|}-1=S \beta_{i \|}^{-\alpha}$ for $S=0.03$ and $\alpha=0.53$. 
A fit of the scaling relationship to the downstream data yields values of .03 for $S$ and .53 for $\alpha$. The value of $S$ is much smaller than for the upstream data. Note that the anisotropy values are considerably smaller in the downstream data versus the upstream data. The ion temperature anisotropy is reduced by collisions, discussed in more detail in the next section, and perhaps by the wave-particle interactions of an instability. As the plasma flows towards the downstream measurement location where the anisotropy is smaller, any anisotropy driven instabilities should shrink in amplitude and growth rate. Thus, it is unlikely that downstream measurements are representative of a strongly driven system near an anisotropy instability threshold.

\subsection{Collisions}

Unlike the magnetosphere, where plasma densities are extremely small and the plasma is essentially collisionless, these experiments are performed in a marginally collisional

plasma. To compare these results to collisionless theory, the effect of varying collision frequencies must be eliminated. Collisions tend to isotropize a plasma by transferring energy from one direction to the another, i.e. velocity space diffusion. Experimentally, the effects of collisions cannot be eliminated but the effects of variations in the collisionality can be minimized by varying the ion temperature anisotropy and parallel ion beta while keeping the relevant collision rates fixed.

Ignoring ion-electron collisions, the rates of change for the perpendicular and parallel temperatures are given by ${ }^{113}$

$$
\frac{d T_{\perp}}{d t}=-\frac{1}{2} \frac{d T_{\|}}{d t}=-v\left(T_{\perp}-T_{\|}\right)
$$

where

$$
\begin{gathered}
v \approx \frac{22 \sqrt{\pi} e^{4} n}{\sqrt{m_{i}}\left(k T_{\|}\right)^{3 / 2} A^{2}}\left[-3+(A+3) \frac{\tan ^{-1}(\sqrt{A})}{\sqrt{A}}\right], \\
A \equiv \frac{T_{\perp}}{T_{\|}}-1 .
\end{gathered}
$$


and $A>0$. For $A<0, \tan ^{-1}(\sqrt{A}) / \sqrt{A}$ is replaced by $\tanh ^{-1}(\sqrt{-A}) / \sqrt{-A} \cdot{ }^{113}$ For the LEIA experimental parameters, the isotropization frequency, $v$, can be approximated by

$$
v \approx \frac{4 \sqrt{\pi} e^{4} n}{\sqrt{m_{i}}\left(k T_{\|}\right)^{3 / 2} A^{9}}
$$

Using the measured plasma temperature and density the ion-ion thermalization frequencies were computed for each data point in Figure 57 (the upstream data). As shown in Figure 60, a subset of all the points for which $v$ varied by less than $11 \%$ could be identified.

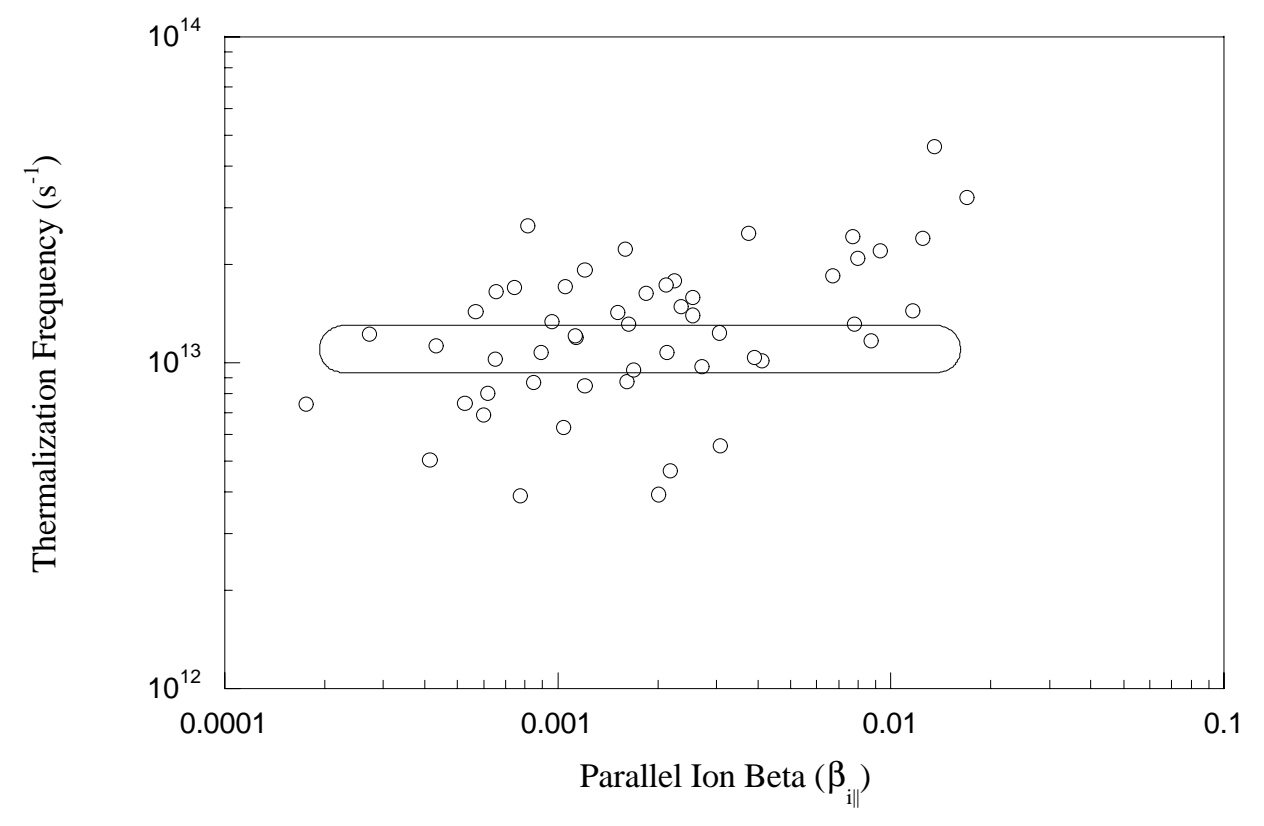

Figure 59: Thermalization frequency versus parallel ion beta for the data set from Figure 57. The circled region are the data selected for the subset of roughly constant ion-ion thermalization rate. 


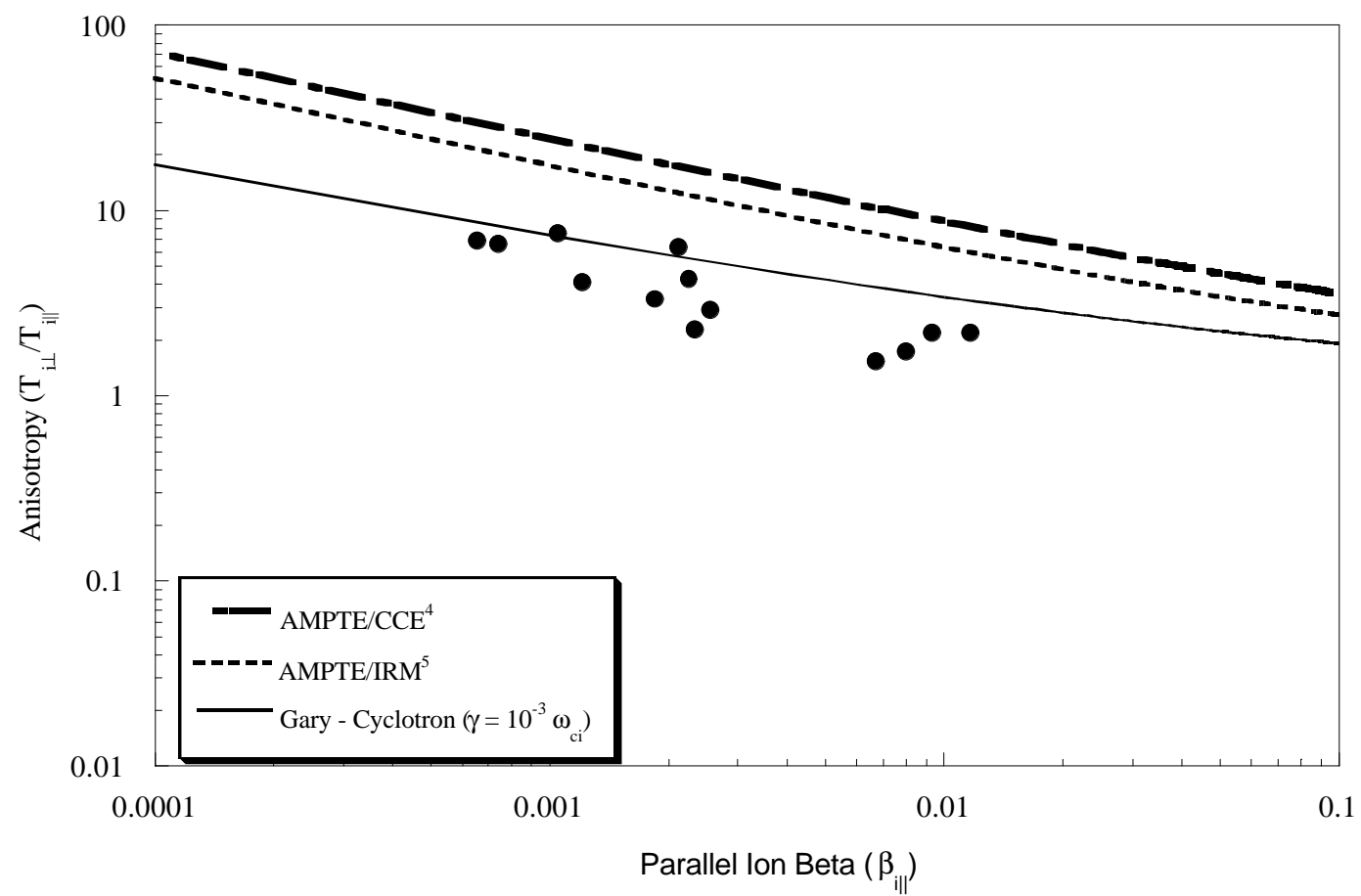

Figure 60: Anisotropy versus beta for a subset of the data with similar ion-ion thermalization frequencies.

The anisotropy versus beta data for the subset of data with similar ion-ion thermalization rates is shown in Figure 63. It is worth noting that if ion-ion collisions were the only source of isotropization, the anisotropy would scale more strongly than $\beta_{i \|}^{-1}$ since both the collision frequency and $\beta_{\mathrm{i} \|}$ increase linearly with increasing density. The data extend over an order of magnitude in beta and a factor of roughly five in anisotropy. The trend remains consistent with the space measurements and theoretical predictions. To eliminate the effects of variation in ion-neutral collisionality, only those data for which the ion-neutral collision frequencies were the same were identified. For ease of analysis, a constant ion-neutral cross section was assumed. For a total energy of $0.4 \mathrm{eV}$ to $1.7 \mathrm{eV}$, such an approximation is reasonable (see Figure 61). Thus, the ionneutral collision frequency is proportional to $\sqrt{T_{i}}$, where $T_{i}^{2}=\left(2 T_{i \perp}^{2}+T_{i \|}^{2}\right) / 3$. Figure 62 shows the ion-neutral collision frequencies versus $\beta_{\mathrm{i} \|}$ for the data of Figure 60. Again, a subset varying by less than $11 \%$ can be identified. Even though this highly constrained 
data spans an even smaller range of parameter space, the same basic relationship between the

ion

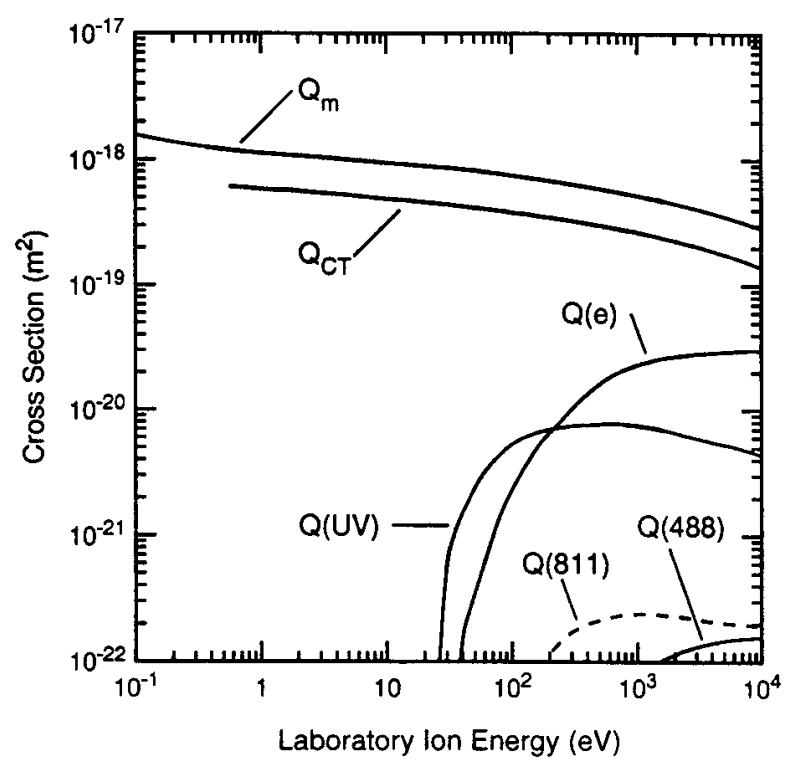

Figure 61: Cross sections for $\mathrm{Ar}^{+}-\mathrm{Ar}$ collisions versus laboratory energy. For the ion energies measured in the experiments, the relevant curves, $Q_{C T}$, the charge transfer, and $Q_{m}$, the momentum transfer are relatively constant. ${ }^{114}$ 


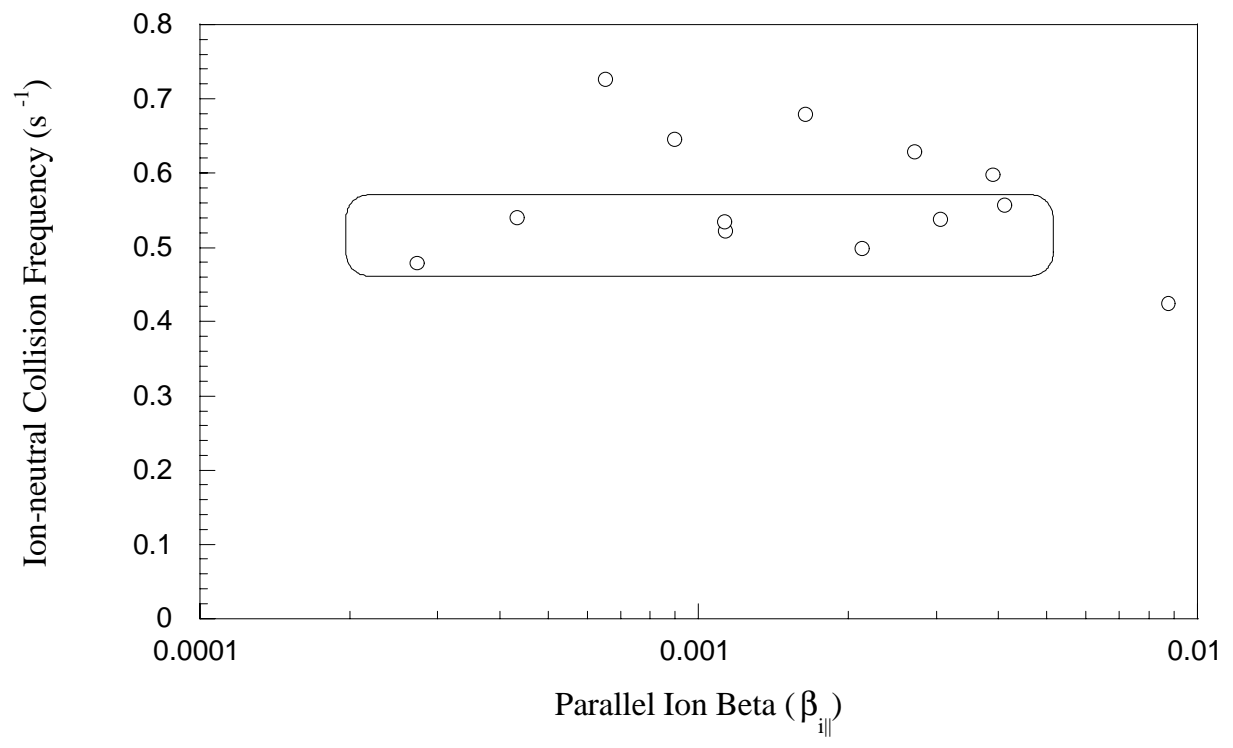

Figure 62: Ion-neutral collision frequency versus parallel ion beta for the subset of data shown in Figure 60. The circled region consists of data with roughly constant ion-neutral collision frequency.

temperature anisotropy and parallel ion beta is observed. A fit to the data yields a value of 0.15 for $S$ and a value of 0.50 for $\alpha$. The data demonstrates that even after the influence of variation in collisionality has been drastically reduced, the inverse correlation of $T_{i \perp} / T_{i \|}$ and $\beta_{\mathrm{i} \|}$ persists. In other words, the inverse scaling of ion temperature and plasma beta appears to be a universal feature of both space and laboratory plasmas. 


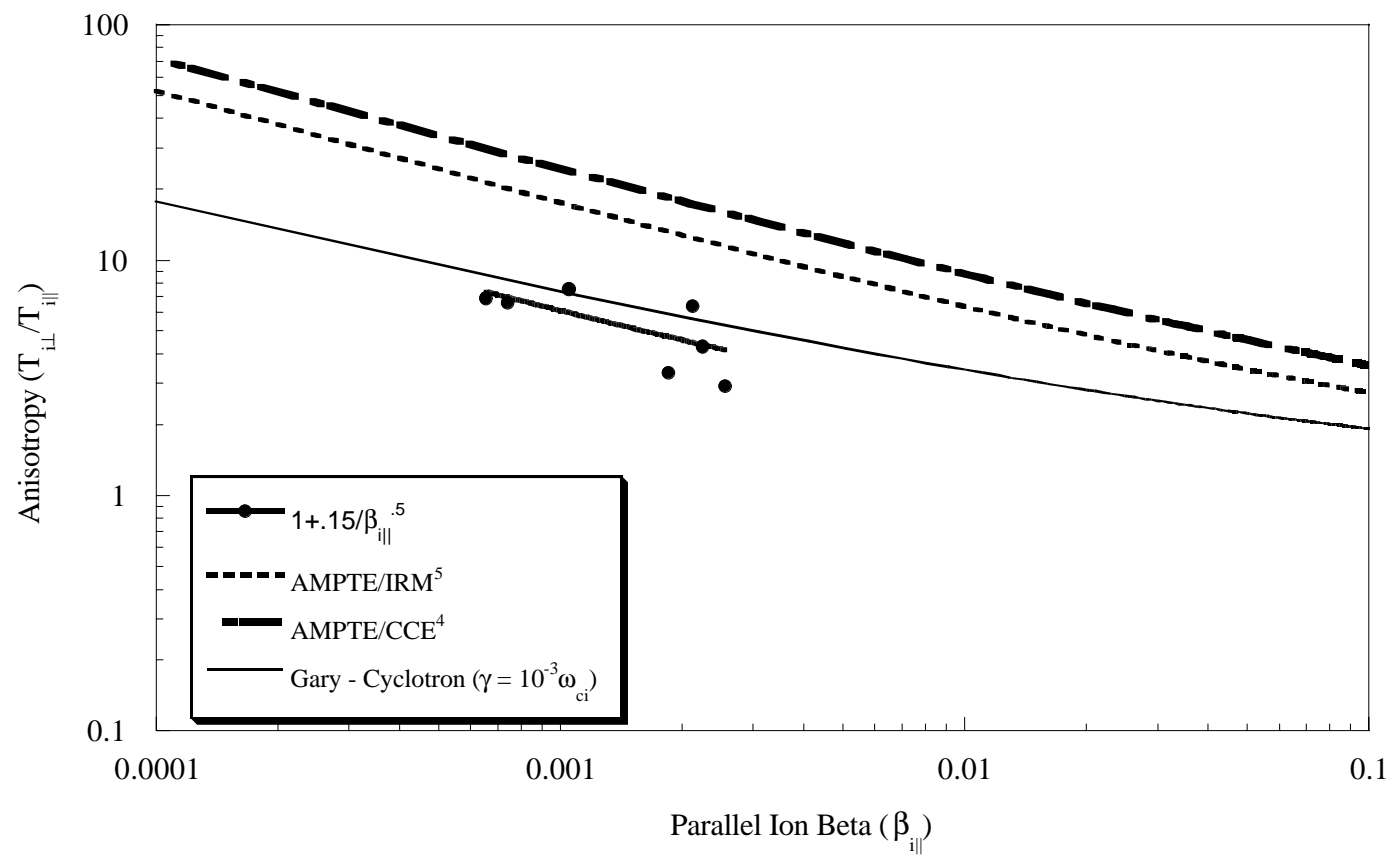

Figure 63: Subset of ion temperature anisotropy and parallel ion beta data for which both the ion thermalization and the ion-neutral collision frequencies varied by less than $11 \%$.

Evidence that ion-neutral collisions are responsible for the cooling of ions can also be extracted from the ion temperature anisotropy data. Because the LIF measurements were performed at two different $z$-positions, it was possible to investigate the spatial evolution of the kinetic energy content of the ions. By summing the parallel and perpendicular temperatures, $T^{2}=\left(2 T_{\perp}^{2}+T_{\|}^{2}\right)^{2}$, at each measurement position, the amount of energy lost by the ions flowing from the upstream to the downstream location can be determined. Since ion-ion collisions conserve energy and ignoring the loss of ion energy to waves for the moment, any measured energy loss should be due to ion-neutral collisions. Ionelectron collisions would only result in ion heating as the electrons are hotter than the ions. The measured energy loss versus the square root of the total ion temperature, effectively the ion neutral collision rate, is shown in Figure 64. Energy loss due to diffusion would scale as $B^{-1}$ or $B^{-2}$, however, the measured energy loss scales linearly with $\sqrt{T_{i}}$, as would be expected for cooling by ion-neutral collisions. 


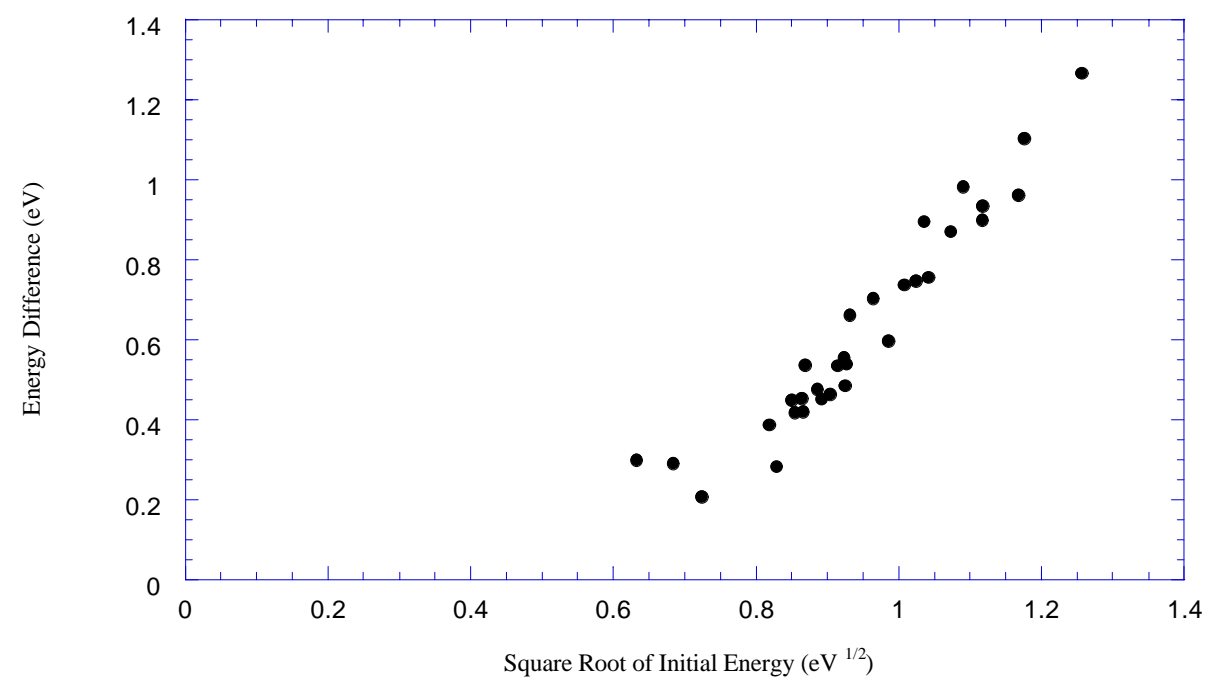

Figure 64: Energy lost by ions traveling from upstream to downstream in LEIA versus the square root of the total initial energy. The linear trend of the data is consistent with a model where the ion energy is lost by ion-neutral collisions.

\subsection{Anisotropy Reduction by waves}

Since the inverse scaling of anisotropy and parallel ion beta persists even for data of near identical collisionalities, another mechanism must be contributing to velocity space diffusion of the ions. Figure 65 shows a typical electromagnetic power spectrum for the plasma conditions used in the ion temperature anisotropy versus ion beta experiments. Although significant wave power exists near the cyclotron frequency, between 0.8 to $2.6 \mathrm{kHz}$, it is important to establish a correlation between ion temperature anisotropy reduction and electromagnetic wave activity. Fortunately, the low frequency waves that might be associated with the ion cyclotron anisotropy instability are suppressed when the ion-heating antenna is turned on. A typical power spectrum with ion heating on is shown in Figure 66. 


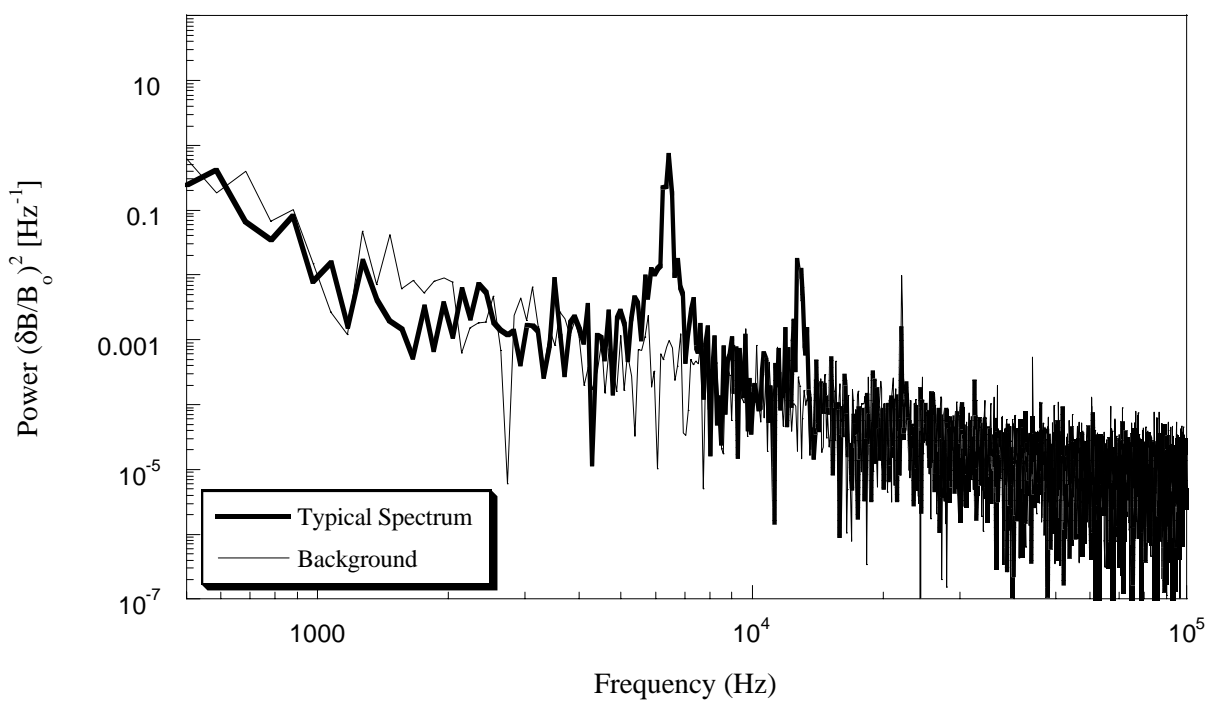

Figure 65: Typical $B_{r}$ power spectrum of electromagnetic waves during ion temperature anisotropy versus $\beta$ experiments. The spectrum was obtained without auxiliary ion heating. The LEIA magnetic field was $66 \mathrm{G}$.

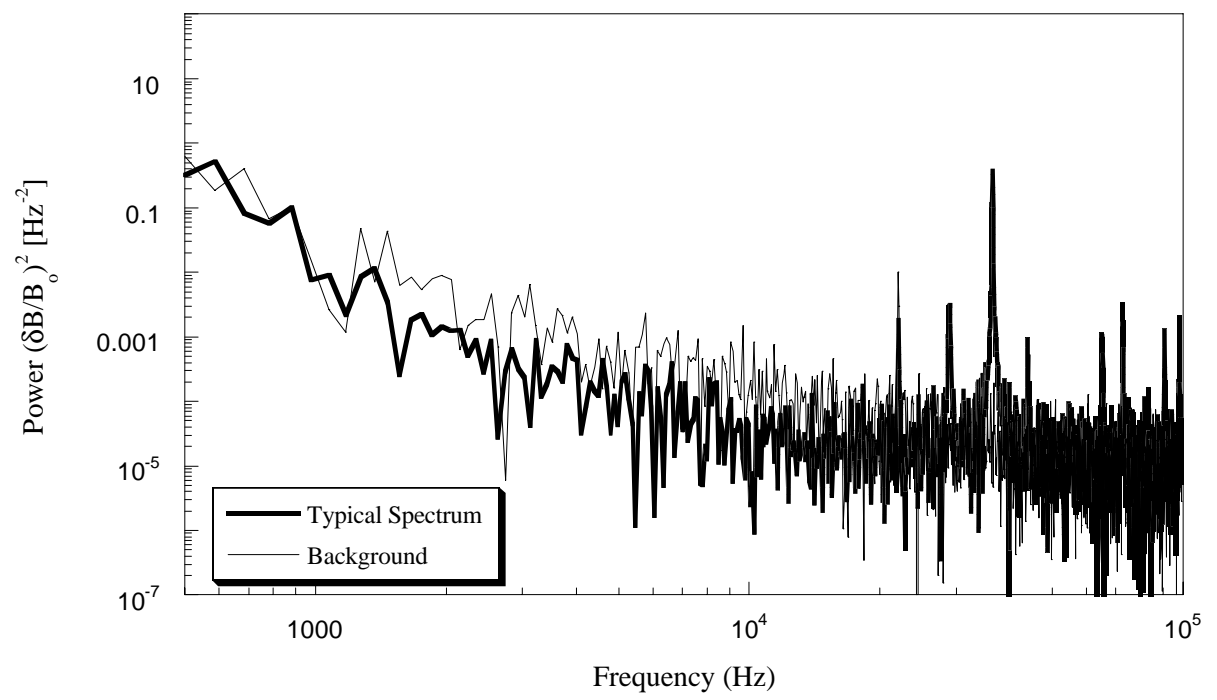

Figure 66: Typical $B_{r}$ power spectrum with ion-heating on. The overall amplitude of low frequency wave activity is much lower than with the heating off. The feature at $33 \mathrm{kHz}$ is the driving frequency of the ion-heating antenna. The LEIA magnetic field was $66 \mathrm{G}$. 
As stated earlier, the LIF measurements in LEIA were performed at two different axial positions. Thus, a measurement of the reduction in anisotropy, or anisotropy ratio can be made. The anisotropy ratio is defined as the ratio of the upstream (closest to the source) anisotropy divided by the downstream (farther from the source) anisotropy. By suppressing the low frequency wave activity, the change in the anisotropy ratio can be measured with and without low frequency electromagnetic wave activity. In this sense, an increase in the anisotropy ratio in the case with wave activity would suggest a correlation between the wave activity and isotropization of the ions.

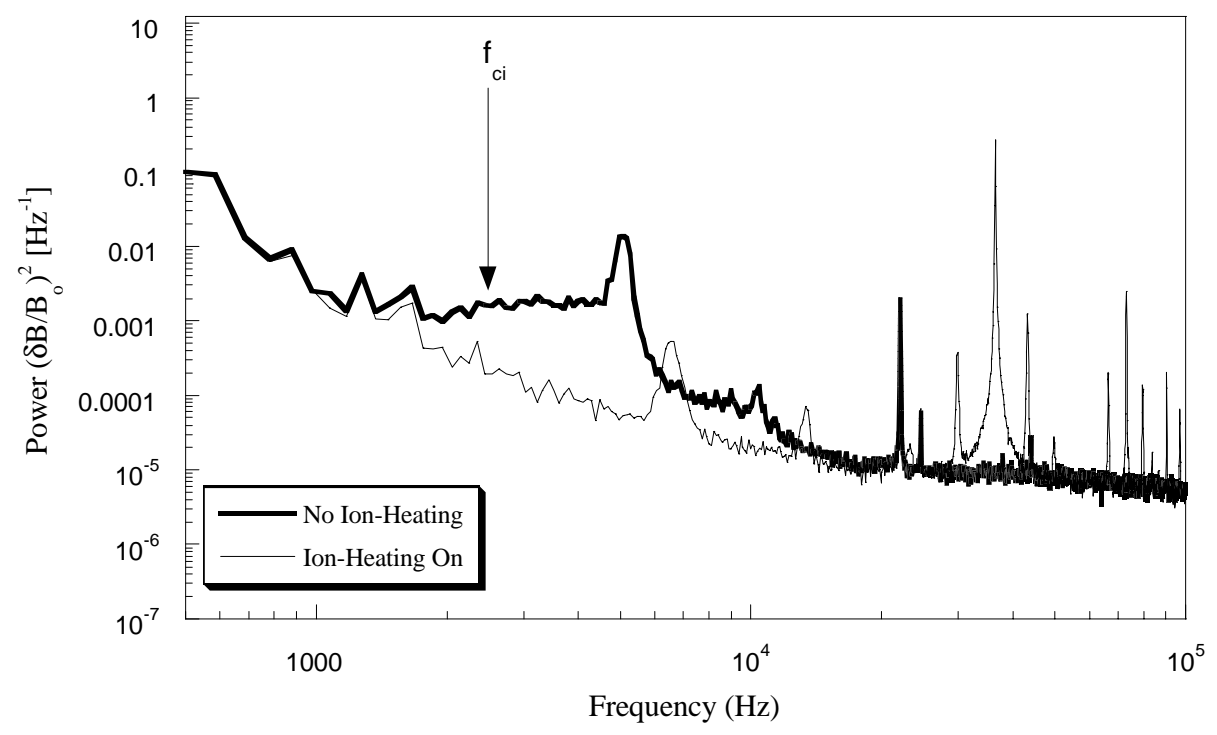

Figure 67: The $B_{r}$ power spectrum for the ion-heating on and ion-heating off cases of the controlled experiment. The low frequency wave power is less when the ion-heating is turned on. The LEIA magnetic field was $65 \mathrm{G}$.

To identify any such correlation, a controlled experiment was performed. At the same magnetic field configuration, LIF measurements were obtained for ion-heating on and ion-heating off cases at both the upstream and downstream locations. By varying source rf power and ion-heating power, the densities and total ion temperatures were kept as similar as possible in both cases to maintain the same ion-ion and ion-neutral collision 
frequencies. Figure 67 shows an overlay of the $B_{r}$ power spectra of the heating on and off cases. When the heating is on, the higher $(6-15 \mathrm{kHz})$ frequencies are shifted, having a lower amplitude and the low frequencies $\left(\omega \sim \omega_{c i}\right)$ are suppressed. The only increases in wave activity are seen at the ion-heating frequency $(\sim 36 \mathrm{kHz})$ and its harmonics.

For the heating-on case, $A_{u p} / A_{\text {down }}=17 / 3=5.7$, while for the heating off case, $A_{u p} / A_{\text {down }}=19 / 2=9.5$. Therefore, with the waves present, the anisotropy decreased more than when the low frequency waves were absent. The heating on case (no waves) may represent the loss of anisotropy due to collisions. If so, then it appears that when the waves are present, the amount of isotropization is increased.

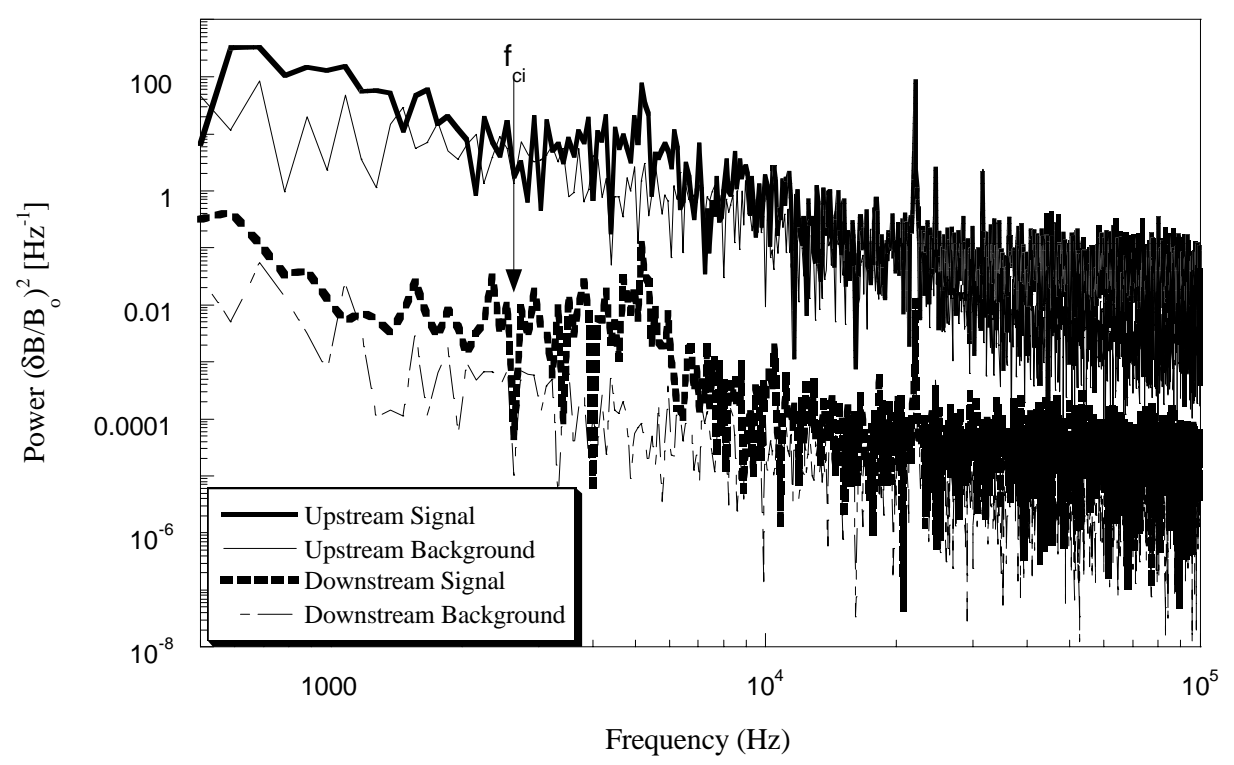

Figure 68: Upstream and downstream $B_{r}$ power spectra. Note the higher level of activity for the upstream case. The LEIA magnetic field was $66 \mathrm{G}$.

Additional evidence in support of the argument that low frequency wave activity is correlated with ion isotropization can be found in the differences between the electromagnetic power spectrum measured at the upstream and downstream positions. Though similar, there are differences between the two power spectra (Figure 68). The 
upstream measurements show a higher level of wave activity. The fluctuations below $\omega_{c i}$ are consistent with the presence of an EMITA instability reducing the ion temperature anisotropy. It is possible that some of the fluctuations above $\omega_{c i}$ are also EMITA instabilities which originate closer to the source where the magnetic field strength is higher (Figure 22). The anisotropy is lower downstream than it is upstream, thus, the amplitude of the fluctuations decreases. 


\section{IDENTIFICATION OF THE WAVES}

Having established that LEIA plasmas exhibit an inverse correlation between the ion temperature anisotropy and the parallel ion beta, and that anisotropy reduction is associated with low frequency wave activity, it is important to determine if the measured characteristics, frequencies and wavenumbers of the waves are consistent with predictions for the ion cyclotron anisotropy or mirror instability. Of particular interest are the waves occurring slightly below the argon cyclotron frequency. For ion cyclotron anisotropy driven instabilities, the wave frequencies should lie below the ion cyclotron frequency. The waves should be transverse with wavenumbers of approximately $k \sim \omega_{p i} / c$ (see Figure 41). ${ }^{91}$ For mirror modes, the waves should be extremely low frequency with wavenumbers of approximately $k \sim \omega_{p i} / 2 c$ (see Figure 44). ${ }^{91}$

\subsection{Wave Power Spectra and Amplitude Measurements}

Typical spectra of the radial magnetic fluctuations for different HELIX magnetic field strengths are shown in Figure 70 to Figure 73. At a particular HELIX value of magnetic field, the spectrum changed from peaked to broadband around $6.5 \mathrm{kHz}$. However, the frequency of this main peak is greater than the highest ion gyrofrequency $(\sim 2.7 \mathrm{kHz})$ in LEIA. Though the higher frequency peaks may be related to the high intrinsic ion temperatures in LEIA, the primary focus is on electromagnetic waves around or slightly below the LEIA ion gyrofrequency. 


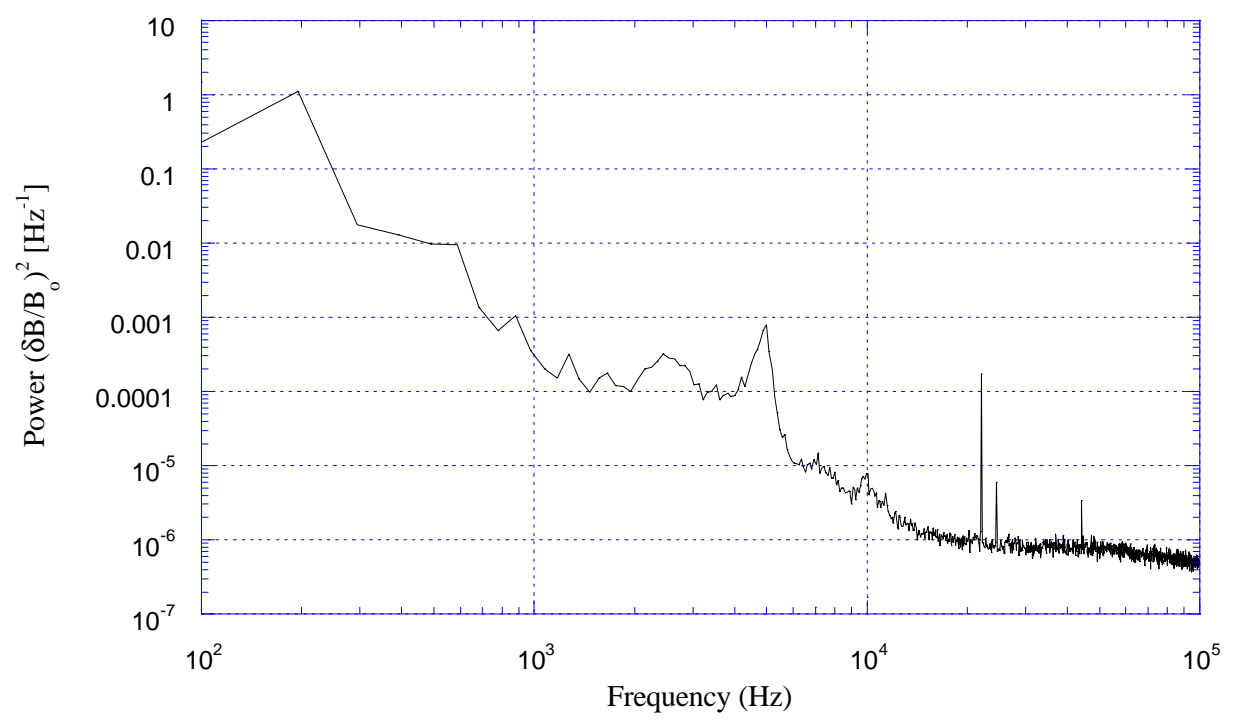

Figure 69: Power spectrum of transverse $\left(B_{\mathrm{r}}\right)$ fluctuations for a HELIX magnetic field of $399 \mathrm{G}$ and a LEIA magnetic field of $65 \mathrm{G}$. There is a peak near $6.5 \mathrm{kHz}$ and a smaller peak at roughly $2.5 \mathrm{kHz}$. The peak at $20 \mathrm{kHz}$ is caused by the pick up of an environmental signal.

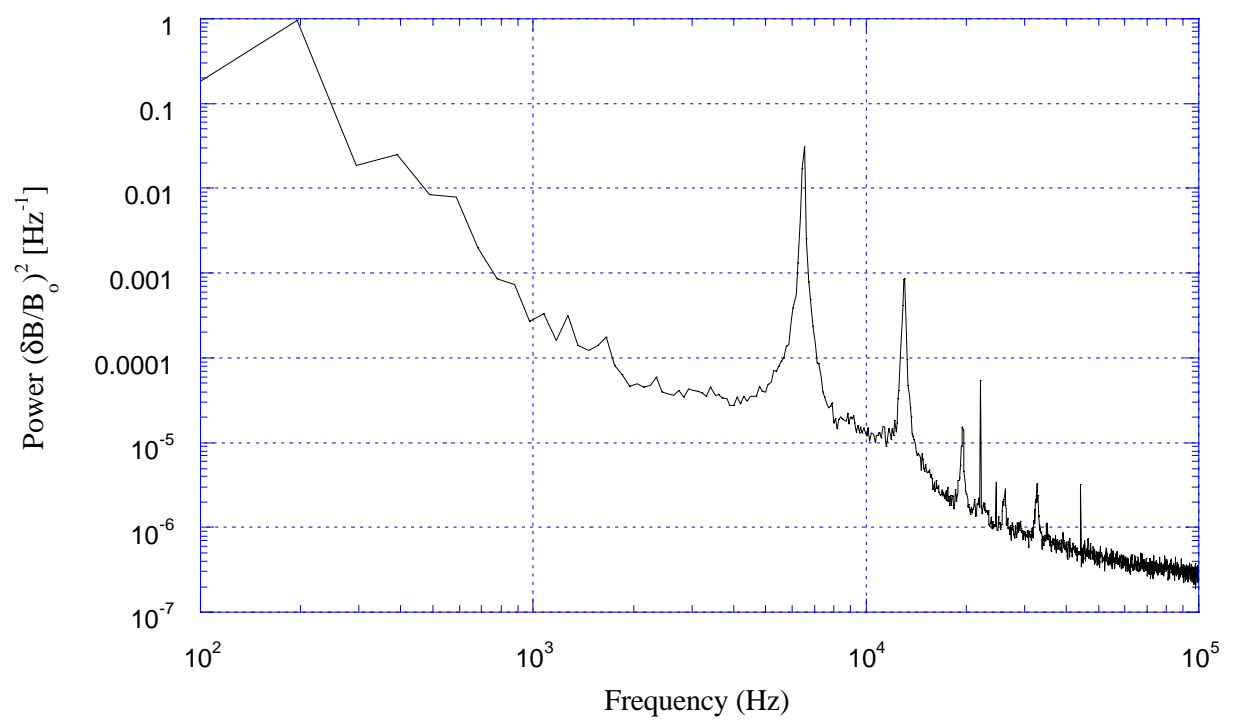

Figure 70: Power spectrum of transverse $\left(B_{r}\right)$ fluctuations for a HELIX magnetic field of $556 \mathrm{G}$ and a LEIA magnetic field of $66 \mathrm{G}$. Note the appearance of the $2^{\text {nd }}$ and $3^{\text {rd }}$ harmonic of the $6.5 \mathrm{kHz}$ wave. 


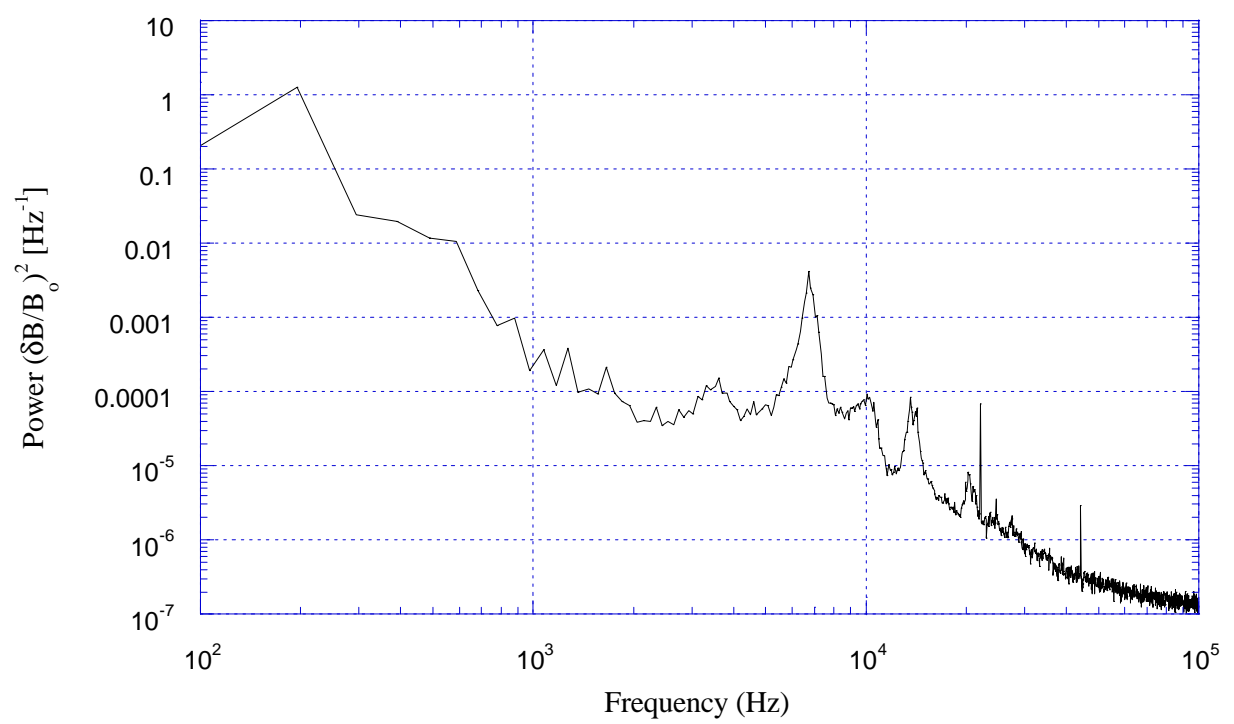

Figure 71: Power spectrum of transverse $\left(\mathrm{B}_{\mathrm{r}}\right)$ fluctuations for a HELIX magnetic field of $792 \mathrm{G}$ and a LEIA magnetic field of $68 \mathrm{G}$. The peak near $7 \mathrm{kHz}$ has begun to broaden and the amplitude is lower than in the previous case.

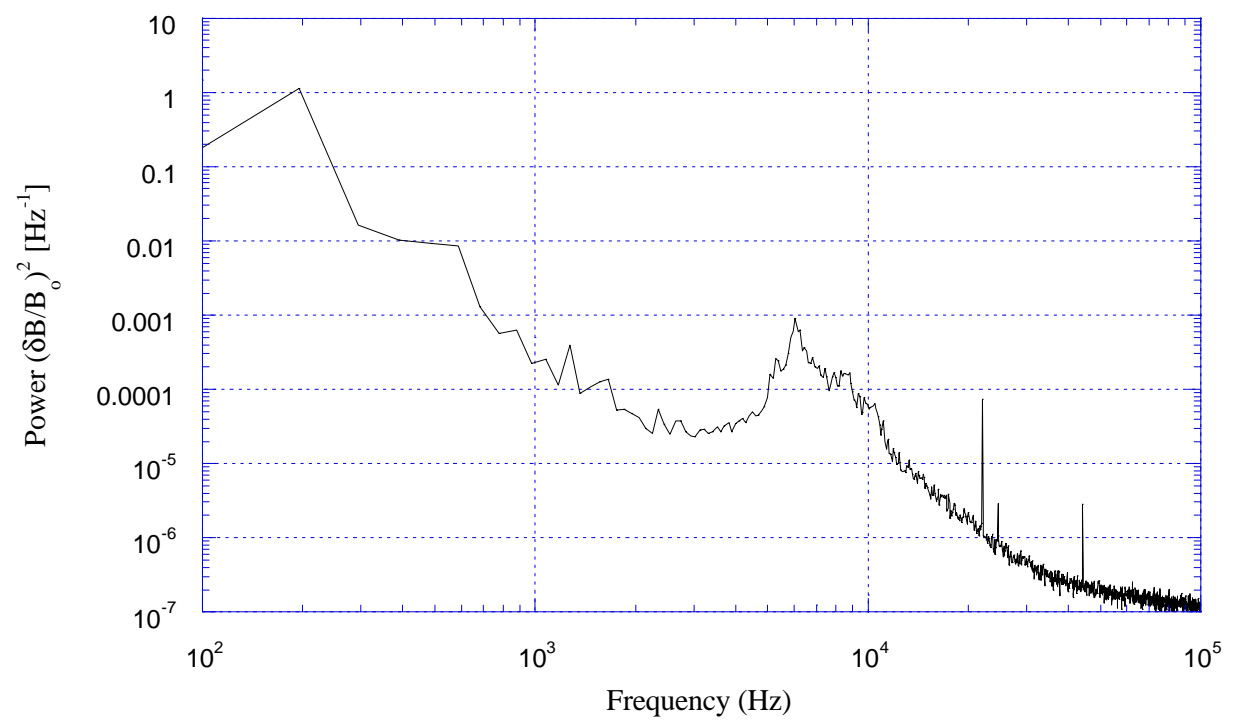

Figure 72: Power spectrum of transverse $\left(\mathrm{B}_{\mathrm{r}}\right)$ fluctuations for a HELIX magnetic field of $1028 \mathrm{G}$ and a LEIA magnetic field of $69 \mathrm{G}$. The roughly $7 \mathrm{kHz}$ peak is broader and has a smaller amplitude than at lower HELIX magnetic fields. 


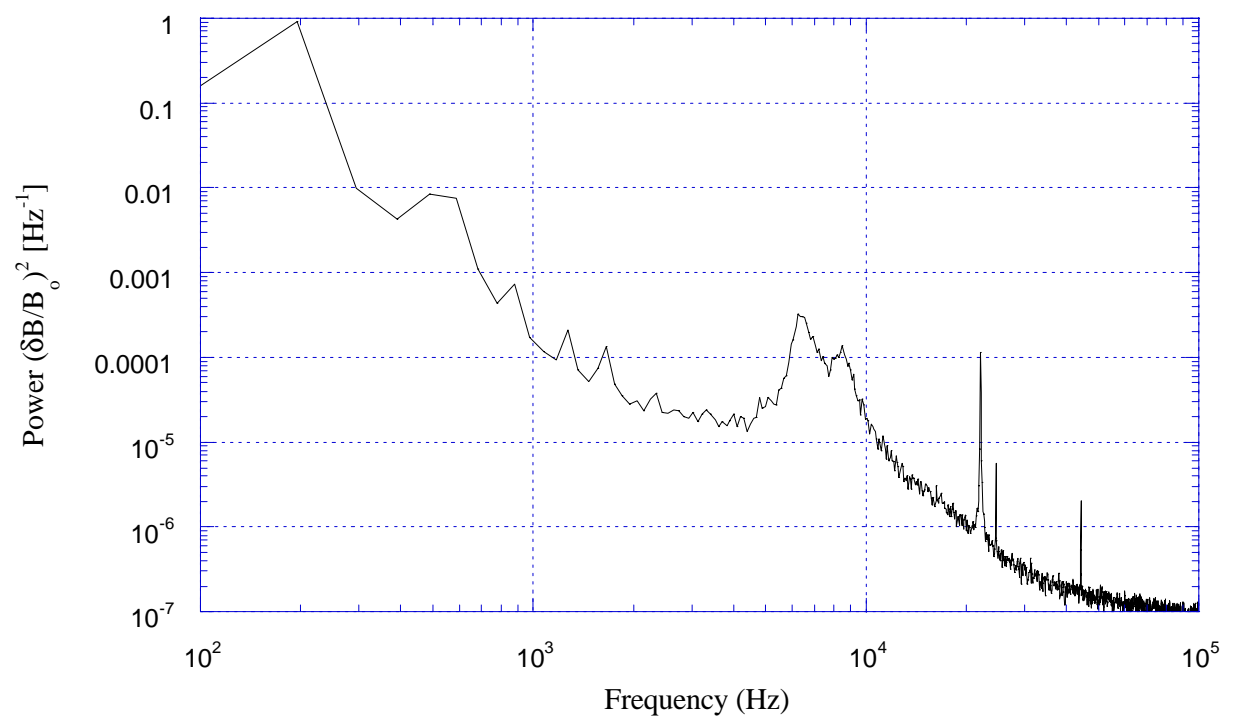

Figure 73: Power spectrum of transverse $\left(\mathrm{B}_{\mathrm{r}}\right)$ fluctuations for a HELIX magnetic field of $1264 \mathrm{G}$ and a LEIA magnetic field of $70 \mathrm{G}$. The roughly $7 \mathrm{kHz}$ peak has a smaller amplitude than previous cases.

Note that the spectral feature at roughly $6.5 \mathrm{kHz}$ changes amplitude and width as the HELIX magnetic field is changed. This suggests that these waves are a HELIX phenomenon and not due to LEIA physics. The magnetic field in LEIA was also varied for each HELIX magnetic field value to identify possible cyclotron features in the electromagnetic power spectrum. Typical results are shown in Figure 70 and Figure 74 through Figure 76. 


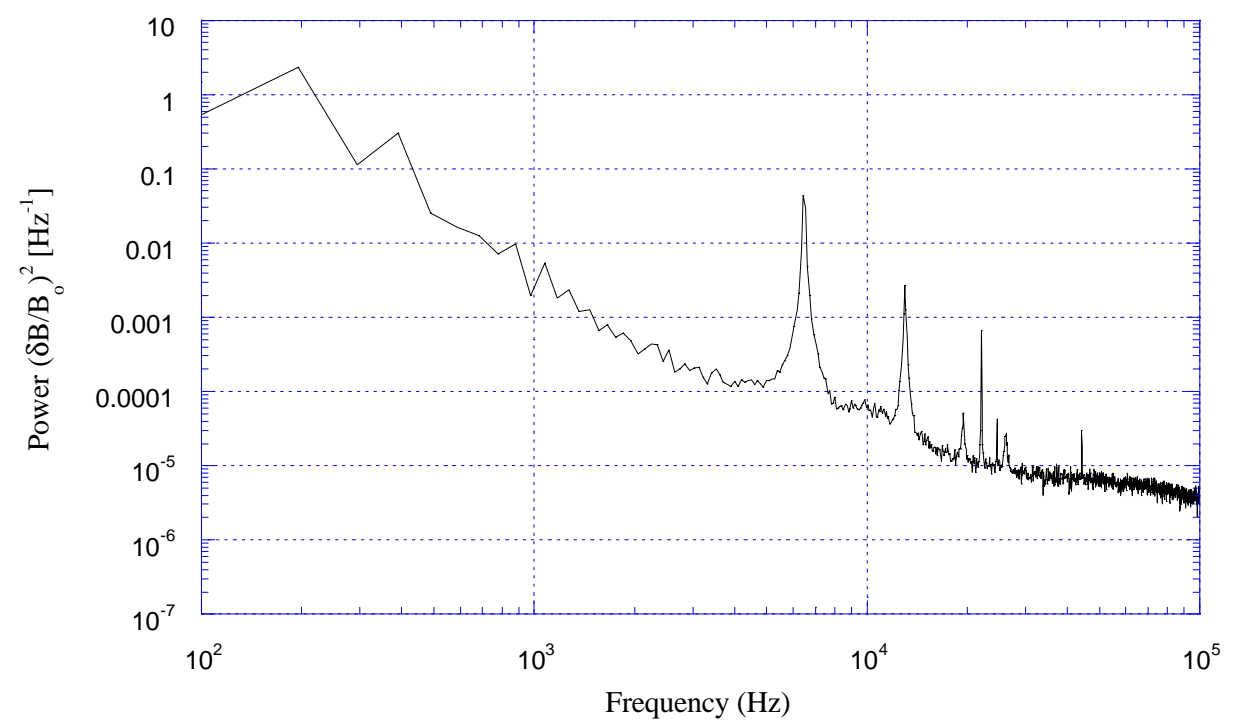

Figure 74: Transverse $\left(\mathrm{B}_{\mathrm{r}}\right)$ spectrum for a HELIX magnetic field of $556 \mathrm{G}$ and a LEIA magnetic field of 18 G. $\beta_{\mathrm{i} \|} \sim .019$.

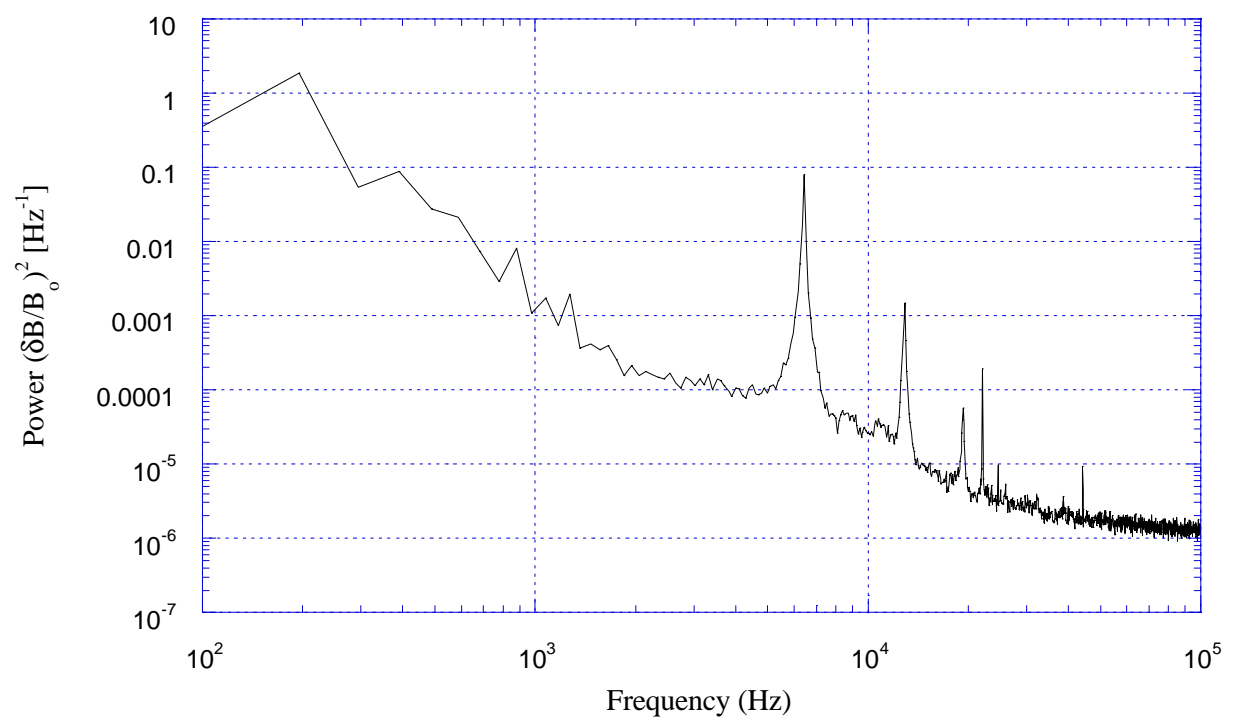

Figure 75: Transverse $\left(\mathrm{B}_{\mathrm{r}}\right)$ spectrum for a HELIX magnetic field of $556 \mathrm{G}$ and a LEIA magnetic field of 35 G. $\beta_{\mathrm{i} \|} \sim .014$. 


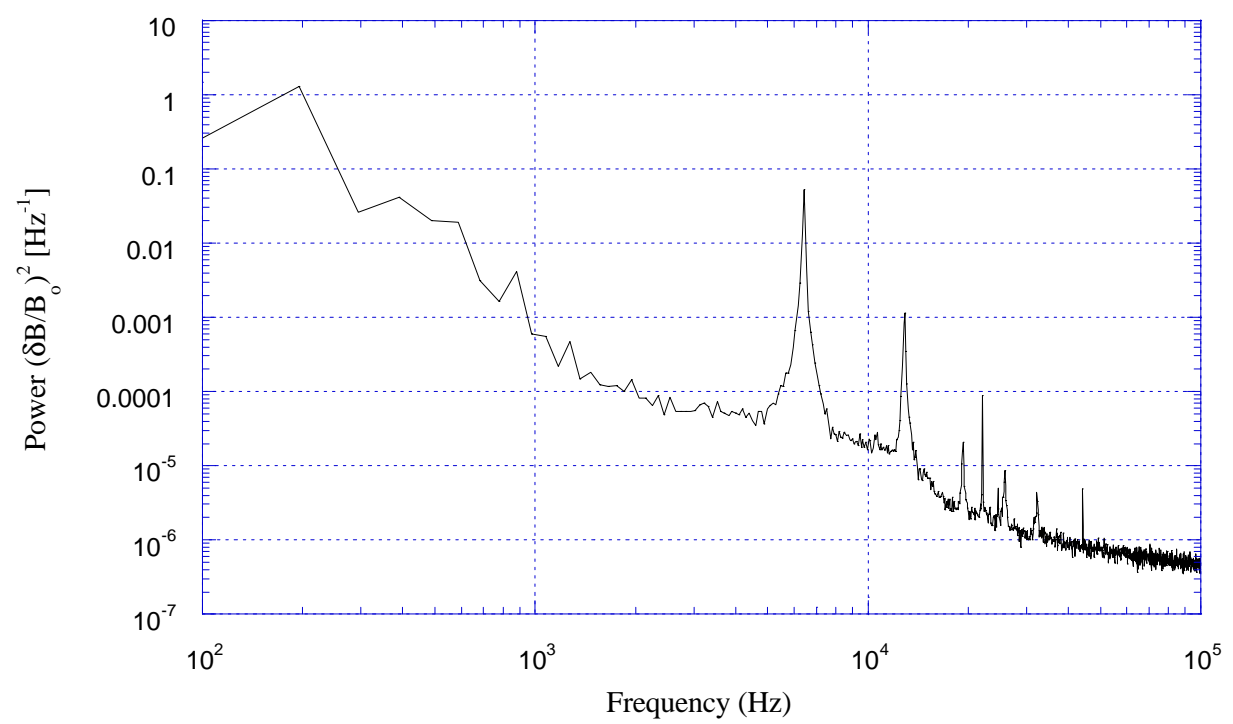

Figure 76: Transverse $\left(\mathrm{B}_{\mathrm{r}}\right)$ spectrum for a HELIX magnetic field of $556 \mathrm{G}$ and a LEIA magnetic field of 51 G. $\beta_{\mathrm{i} \|} \sim .008$.

As the LEIA magnetic field is varied, the large spectral at $6.5 \mathrm{kHz}$ does not change in frequency or width. This supports the conclusion that these waves are generated in HELIX and are simply detected in LEIA. However, there is a clear decrease in power at lower frequencies $(1-2 \mathrm{kHz})$ as $\beta_{\mathrm{i} \|}$ decreases. The fluctuation power integrated from 0.5 $\omega_{\mathrm{ci}}$ to $1 \omega_{\mathrm{ci}}$ and normalized by $\Delta \omega$ is plotted versus $\beta_{\mathrm{i} \|}$ in Figure 77 . The data were fit to a exponential of the form $P=S_{B} e^{\alpha_{B}}$, where $P$ is the total integrated power and $S_{\mathrm{B}}$ and $\alpha_{\mathrm{B}}$ are fitting parameters. The trend of the wave power as a function of $\beta_{\mathrm{i} \|}$ is significantly different than that found in simulations. ${ }^{2}$ Gary reported a power law dependence of $\left(\delta B / B_{o}\right)^{2}$ on $\beta_{\mathrm{i} \|}$ in 1-D hybrid simulations of proton temperature anisotropy instabilities (Figure 78a). ${ }^{2}$ Similar power law relationships have also been reported for 2-D simulations (Figure $78 \mathrm{~b})^{2}$ 


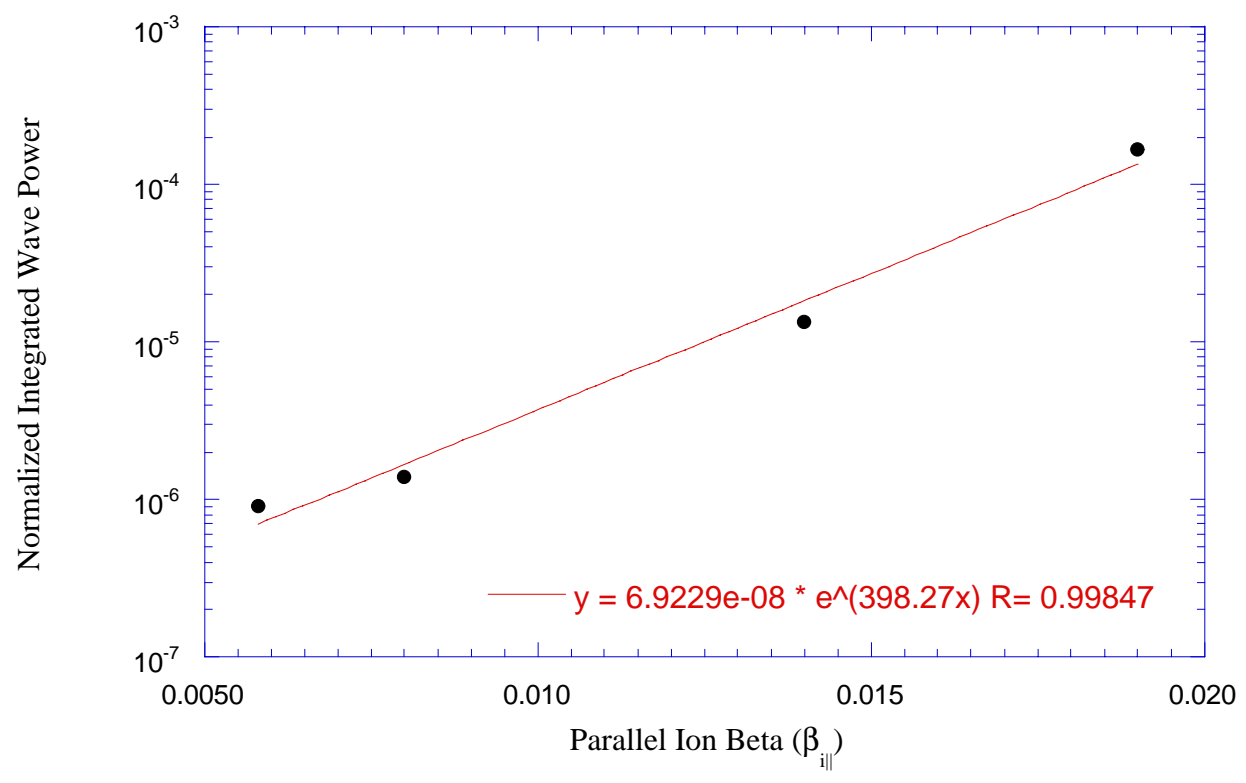

Figure 77: Integrated wave power for 0.5 to $1.0 \omega_{c i}$ normalized by $\Delta \omega$ and normalized by the total power in the entire power spectrum for the LEIA magnetic field scan cases. Note, at low values of beta, the data levels off to the noise level, where the low frequency fluctuations are difficult to resolve from the background level. The fit indicates a scaling of wave power with $\beta_{\mathrm{i} \|}$ that goes roughly as $e^{398 \beta}$, much stronger than the power law scalings seen in 1-D and 2-D simulations. ${ }^{2}$

Although there is a clear increase in the low frequency wave power with $\beta_{\mathrm{i} \|}$. At low values of beta, the normalized integrated wave power levels out. This represents the noise level, where the low frequency fluctuations are difficult to detect from the background fluctuation levels. There are some key differences between the simulations and these experiments. In the simulations, the initial ion temperature anisotropy is held constant for all values of beta. In the experiment, the ion temperature anisotropy changes by a factor of four. Integrated broadband wave activity was considered in the experimental cases, while the simulation results are based on the amplitude scale of a single spectral feature. It is clear from the experimental data that the low frequency wave activity increases in amplitude with increasing beta and that the dependence on beta is much stronger than predicted by the simulation. More realistic simulations are needed before the wavenumber measurements can be quantitatively compared to the models. 
The electromagnetic waves observed in LEIA are clearly transverse waves. Figure 79 shows a comparison between the power spectra from a $B_{\mathrm{r}}$ coil and a $B_{\mathrm{z}}$ coil. Unlike the $B_{\mathrm{r}}$ power spectrum, there is no enhanced low frequency wave activity along the equilibrium field. Preliminary measurements with an electrostatic probe show no evidence of electrostatic wave activity in the frequency region around the ion cyclotron frequency in LEIA where electromagnetic waves are found. Since the waves observed in LEIA are dominantly electromagnetic and transverse, mirror modes can be eliminated as a candidate to explain the low frequency wave activity.
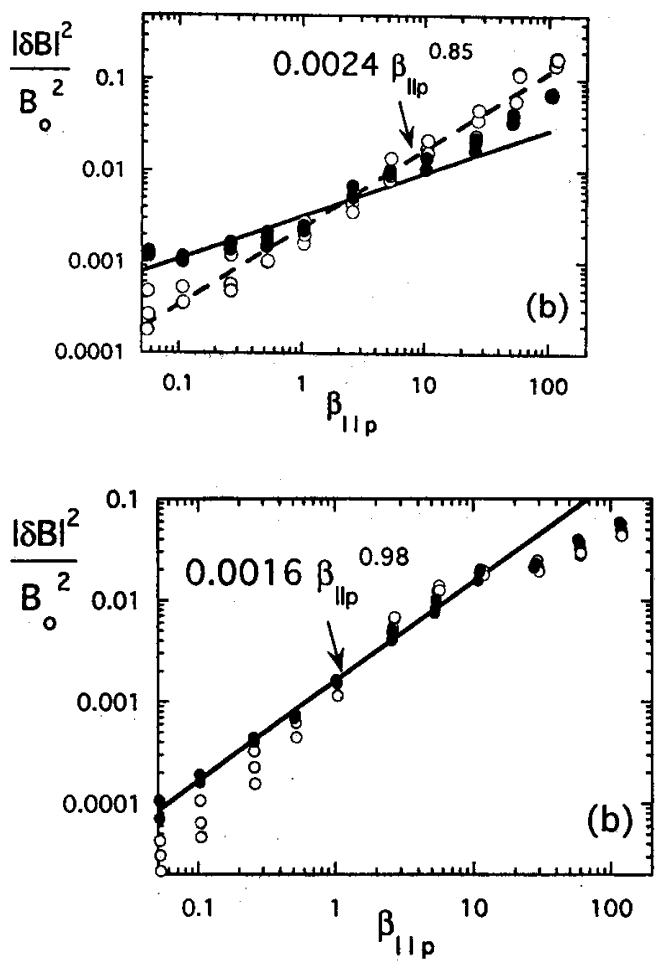

Figure 78: a) The scaling of the wave power seen in the 1-D simulations. ${ }^{2}$ b) The scaling of the wave power versus beta seen in the 2-D simulations. ${ }^{2}$

Another possible source of low frequency electromagnetic waves are electromagnetic drift waves. Such drift waves should be strongest in the region of largest density gradient. As shown in Figure 80, the LEIA density profile is peaked on axis for these experiments. The normalized density gradient $\delta n / n$ peaks at $\pm 5 \mathrm{~cm}$. $B_{\mathrm{r}}$ power spectra for two different radial positions are shown in Figure 81. For the low frequencies, $1-2 \mathrm{kHz}$, there is little 
change in the wave power over a change in radius of $24 \mathrm{~cm}$. In addition, even electromagnetic drift waves should have a significant electrostatic component. The absence of significant electrostatic wave activity in the ion cyclotron frequency range further supports the conclusion that the observed waves are not drift waves.

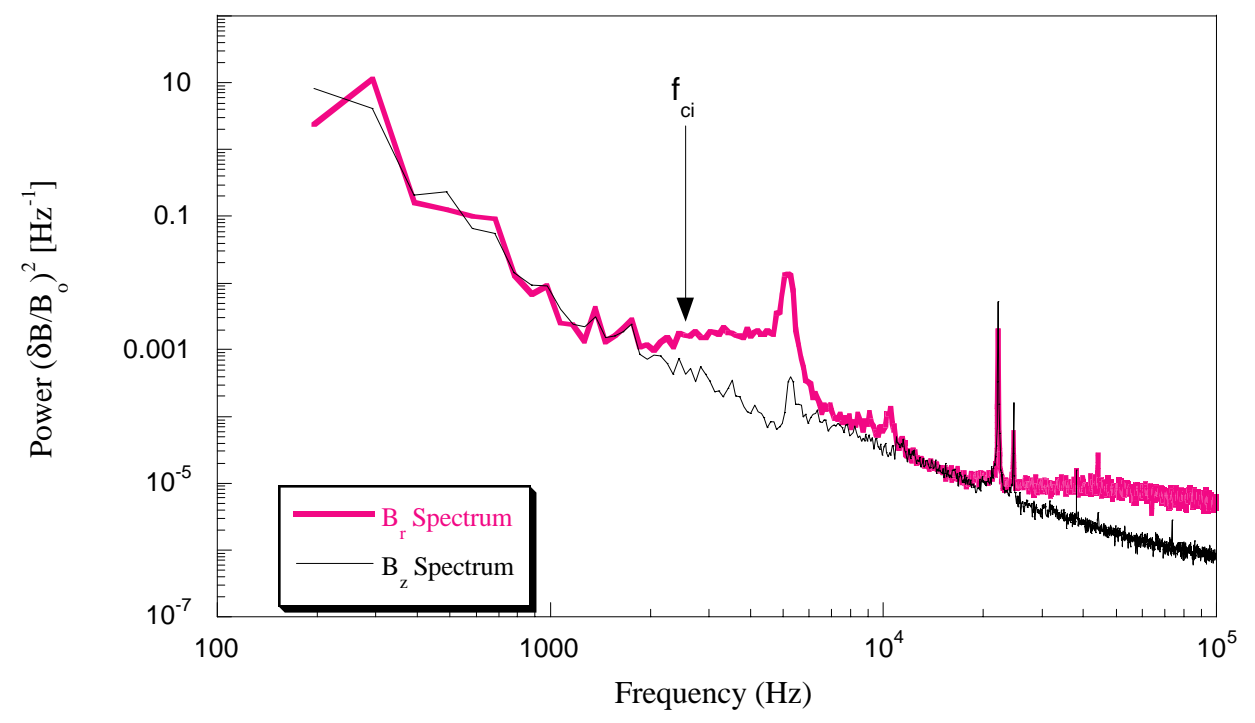

Figure 79: $\mathrm{A} B_{\mathrm{z}}$ power spectrum from the controlled experiment. There are no prominent features visible, unlike a $B_{\mathrm{r}}$ spectrum (Figure 65). The feature around $20 \mathrm{kHz}$ is an artifact that is present when there is no plasma. 


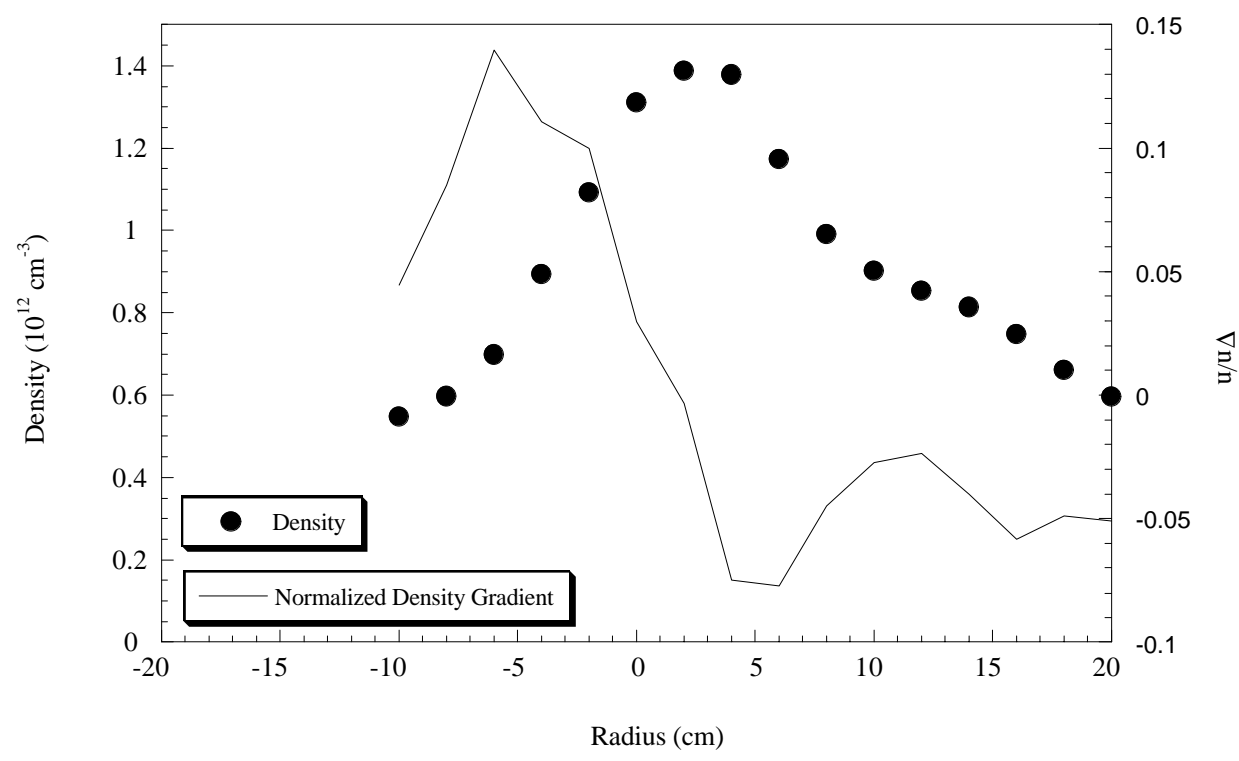

Figure 80: The density profile and normalized density gradient profile for LEIA for a magnetic field of $35 \mathrm{G}$. The normalized gradient peaks at $\pm 5 \mathrm{~cm}$.

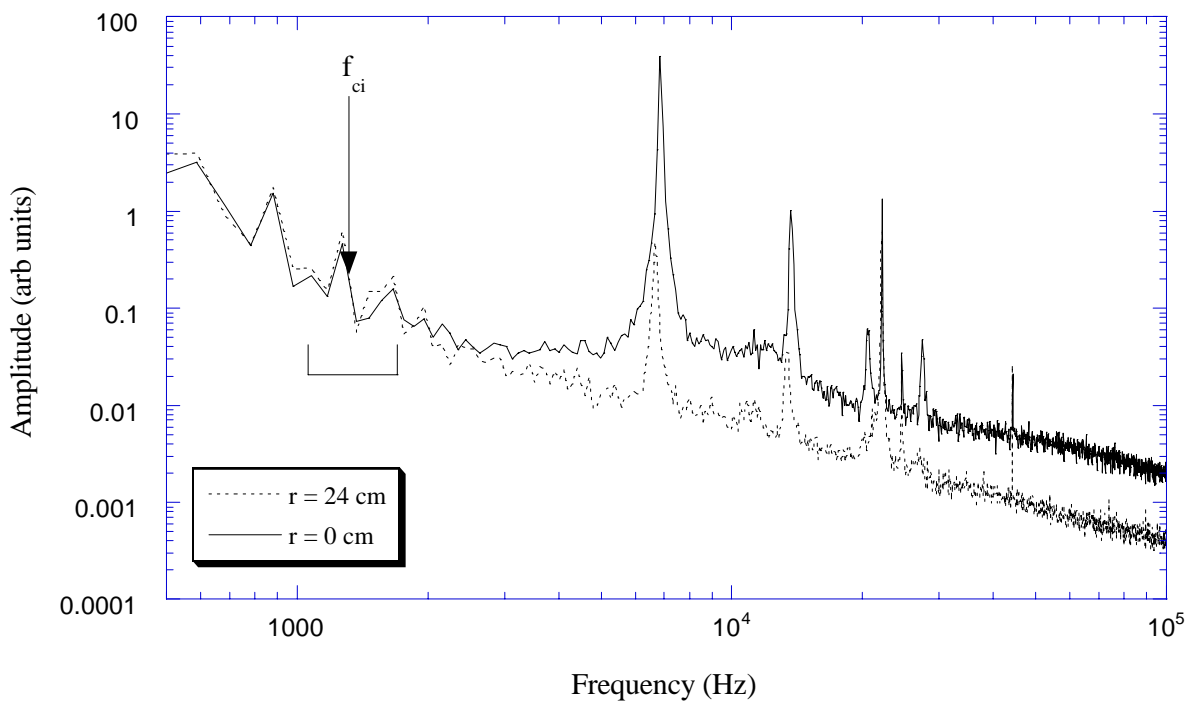

Figure 81: Comparison of the $B_{\mathrm{r}}$ fluctuation spectra at two different radial positions. 


\subsection{Wavenumber Measurements}

Figure 82 is a typical frequency versus wavenumber plot (referred to as a wavenumber spectrum or K-spectrum) for one of the ion temperature anisotropy versus beta experimental configurations. The log of the amplitude is plotted versus frequency and wavenumber. There is one large feature from roughly 5350 to $9070 \mathrm{~Hz}$ and -0.01 to $0.005 \mathrm{~cm}^{-1}$. A positive value of $k$ corresponds to waves traveling from HELIX to LEIA. The $k$ resolution varies from each pair of coils selected, but for the transverse coil pair (coils 2 and 10) used for the majority of the results described in this section, the wavenumber resolution, as defined in Section 3.3.2 is $6 \times 10^{-5} \mathrm{~cm}^{-1}$.
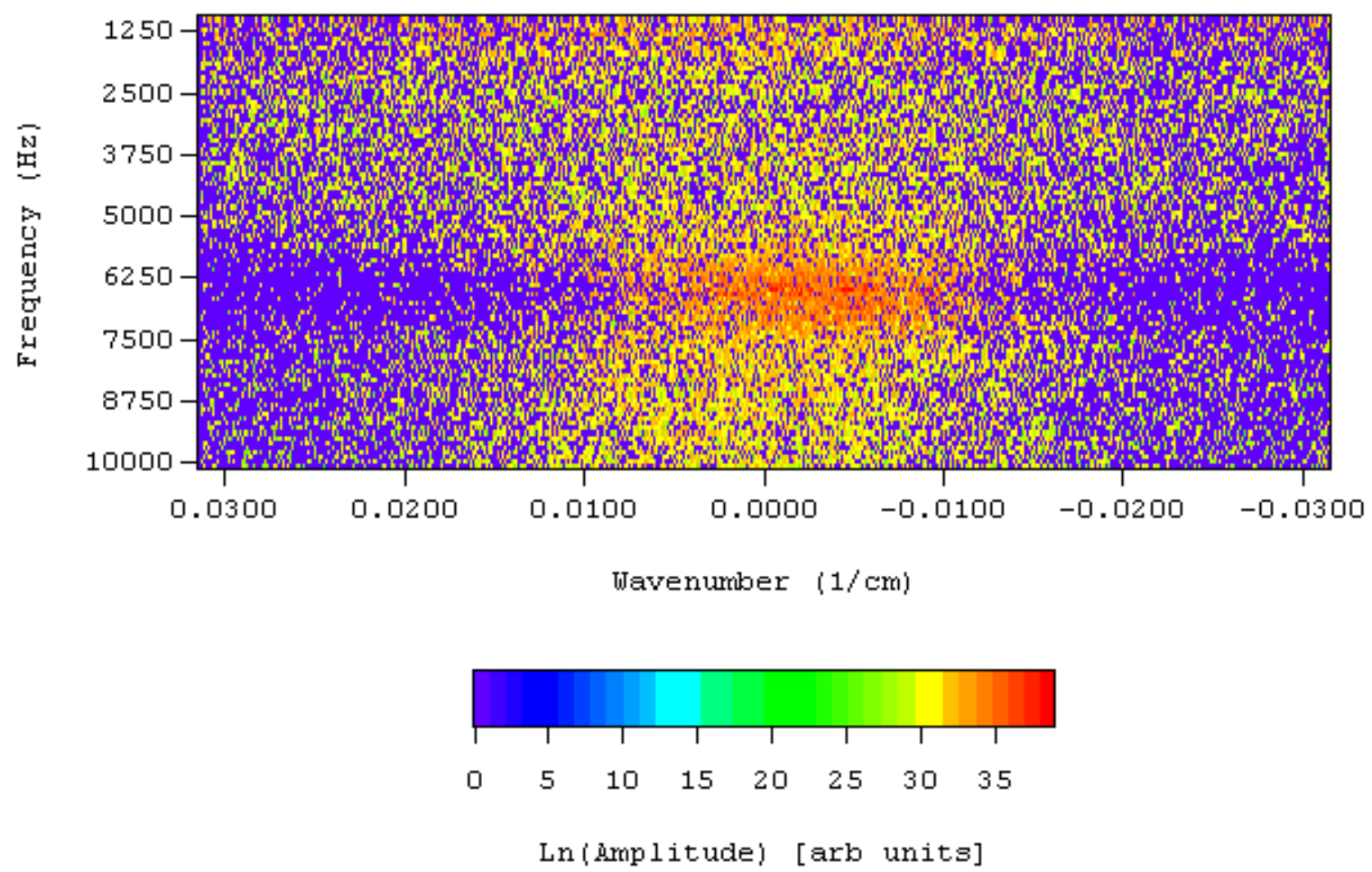

Figure 82: A typical K-spectrum. The parameters are Helix $B=1264 \mathrm{G}$, LEIA $B=55 \mathrm{G}$. Only the frequencies between 1 and $10 \mathrm{kHz}$ are shown. A prominent feature is centered at roughly $6250 \mathrm{~Hz}$. $B_{\mathrm{r}}$ coils 2 and 10 are used, so the data represent parallel wavenumbers for transverse fluctuations. 
To reduce the statistical noise in the wavenumber plot, the data are smoothed with a running 2-D boxcar average three pixels wide and three pixels long. Figure 83 shows the results of the smoothing routine applied to the data of Figure 82. To obtain Figure 83, five smoothing passes were performed. The smoothing accentuates the coherent features in the data and smoothed plots were used to identify interesting cases for further study. To determine the wavenumbers associated with a specific frequency or frequency range, the amplitudes are summed over the frequency range for each wavenumber. To ensure that peaks appearing in the smoothed data are not artificially created, the wavenumber spectra for different frequency bands are generated using both the smoothed and unsmoothed data.
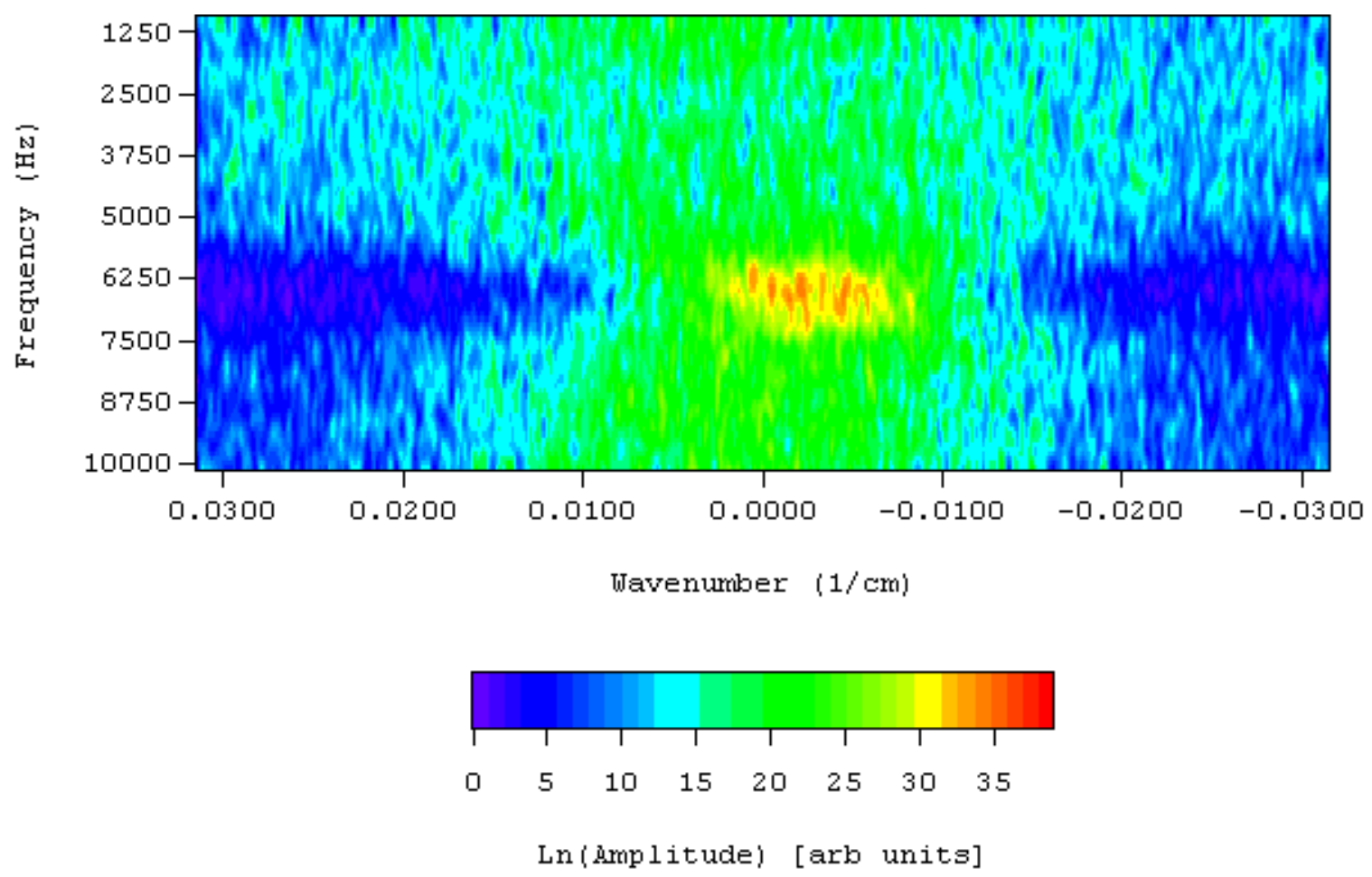

Figure 83: The same data as Figure 82, but smoothed to reduce the background level. There is one very prominent feature on this graph.

The summed amplitude versus wavenumber for frequencies $1 \mathrm{kHz}$ to $10 \mathrm{kHz}$ of the data shown in Figure 83 is shown in Figure 84 for unsmoothed data and in Figure 85 for smoothed data. Although for a slightly lower magnetic field, the frequency spectrum for this case is similar to that shown in Figure 73 - a broad, turbulent regime centered 
roughly at $7 \mathrm{kHz}$. The wavenumber spectra plots of Figure 84 and Figure 85 are consistent with a turbulent interpretation. There are two broad regions of moderate $k$ waves traveling away from the probe. The wavenumber spectrum decreases at higher $k$, consistent with damping at small spatial scales. Both the smoothed and unsmoothed spectra show indications of specific wavenumber peaks at low $k$ (see Figure 84 and Figure 85).

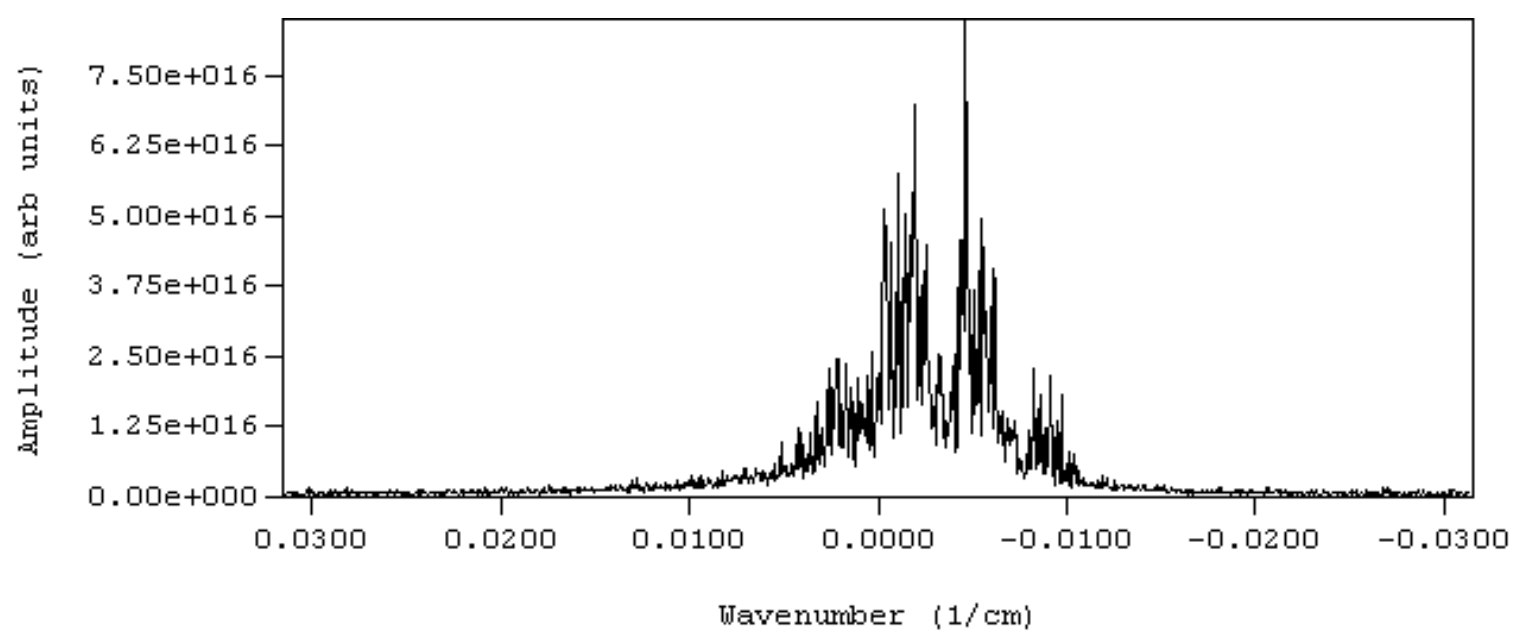

Figure 84: Wavenumber summation over the frequency range of $1 \mathrm{kHz}$ to $10 \mathrm{kHz}$ for the data shown in Figure 82.

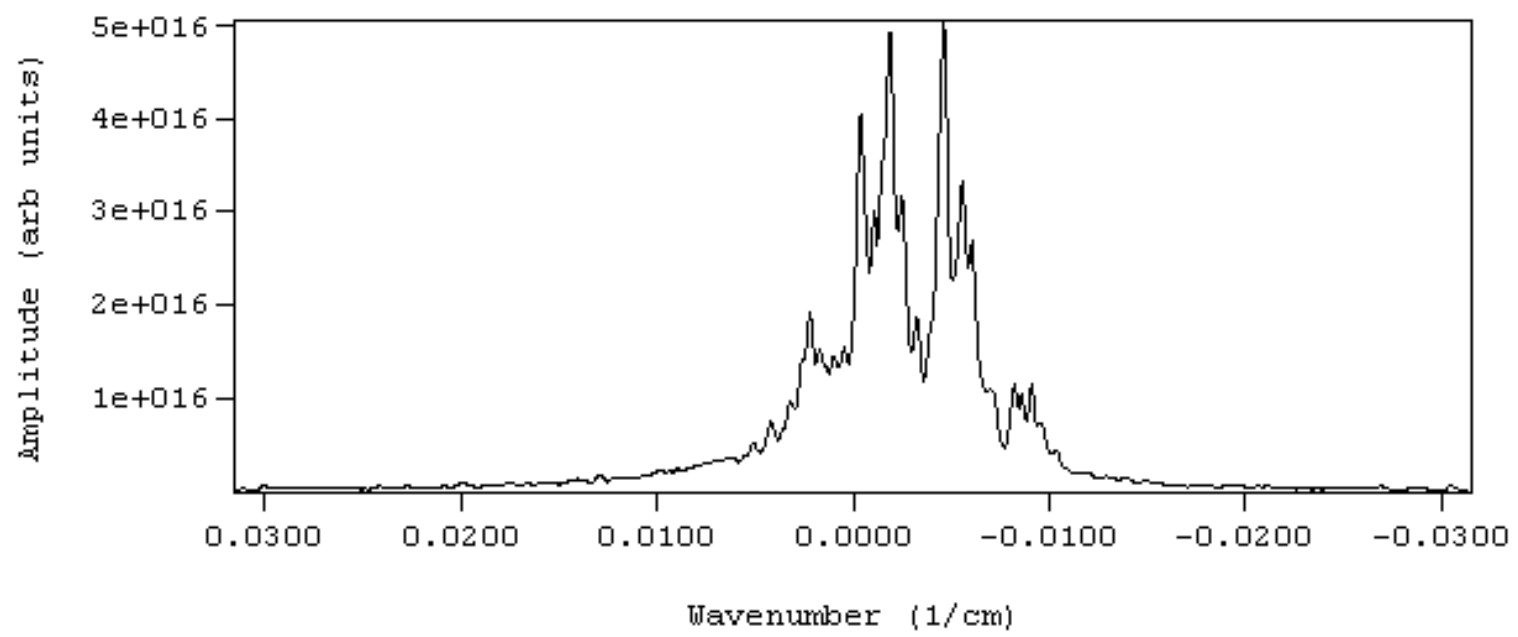

Figure 85: Wavenumber summation for the smoothed data shown in Figure 83 for the frequency range of $1 \mathrm{kHz}$ to $10 \mathrm{kHz}$. 
Smoothing does spread the amplitudes across a wider $k$ range, however, the primary wavenumber peaks are consistent with the unsmoothed data. Clearly, some information is lost by smoothing and only the more dominant features remain. Since it is easier to initially identify regions of enhanced amplitude in smoothed 2-D wavenumber spectra, the wavenumber summation information will be of unsmoothed data and the 2-D wavenumber spectra plots will be of smoothed data.

To determine if the measured wavenumbers correspond to the ion cyclotron anisotropy instability, the data was compared to predictions from the approximate dispersion relation, equation (122), derived by Davidson and Ogden. ${ }^{94}$ Although this approximation assumes $T_{\|}=0$, it can be used to calculate a lower limit on the wavenumber. Since most of the data has an intense feature between 5 and $7 \mathrm{kHz}$, the wavenumbers associated with this feature will be discussed first. However, as the cyclotron frequency in the center of LEIA is no greater than $2.7 \mathrm{kHz}$, these waves cannot be Alfvén ion cyclotron waves in LEIA. Waves in the frequency range of 0.5 to $2.5 \mathrm{kHz}$ will be examined in detail later in this section.
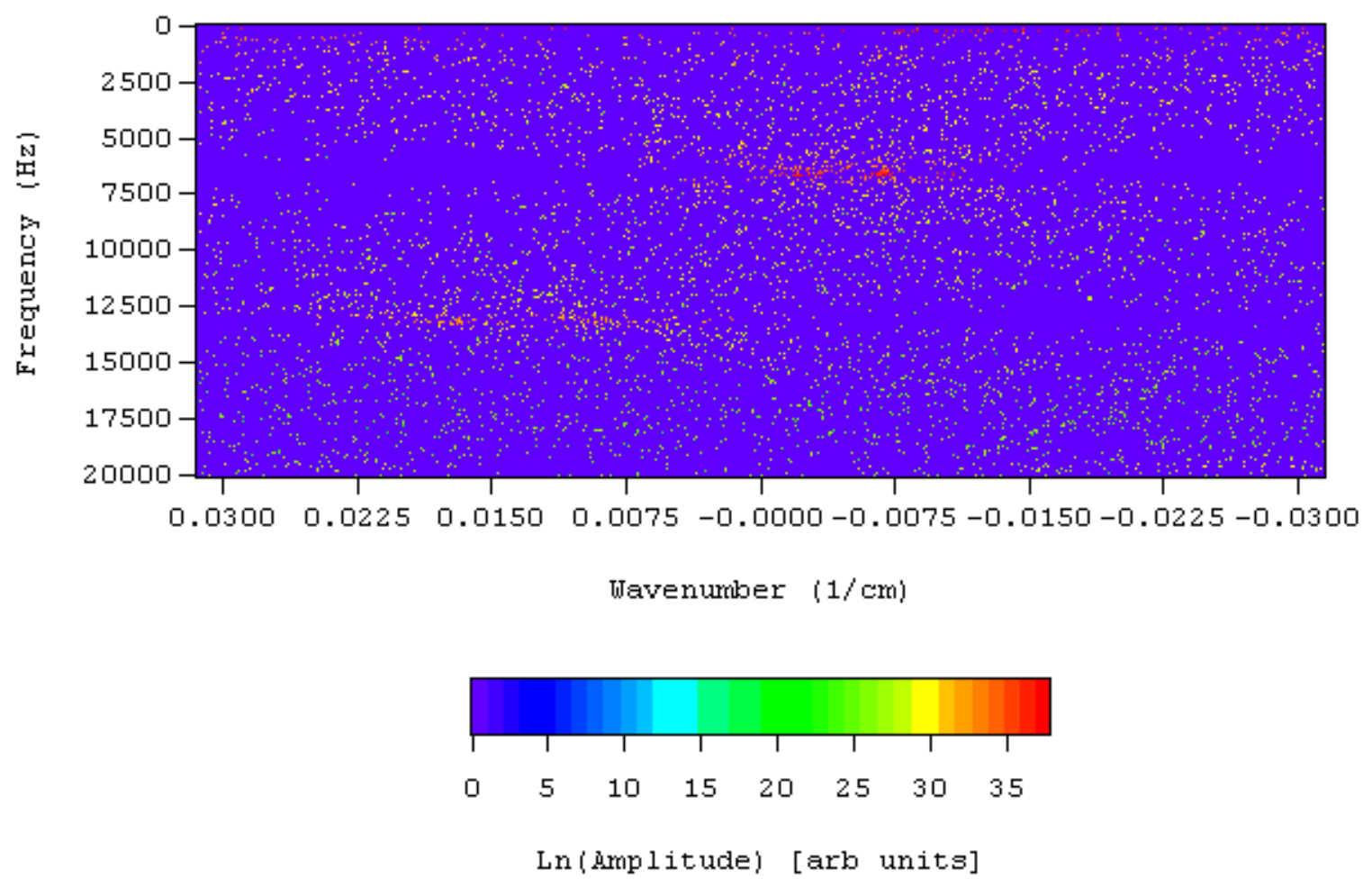

Figure 86: The wavenumber spectrum from a pair of transverse, $B_{\mathrm{r}}$, coils for magnetic fields of $556 \mathrm{G}$ in HELIX and $66 \mathrm{G}$ in LEIA. 
Figure 86 is a 2-D wavenumber spectrum for one of the cases used in the anisotropy versus ion beta experiment. There are two distinct frequency bands across a range of wavenumbers. These are roughly at $6.5 \mathrm{kHz}$ and its harmonic, $13 \mathrm{kHz}$. The wavenumbers in three frequency regions will be examined: 0.5 to $2 \mathrm{kHz}, 4.5$ to $8.5 \mathrm{kHz}$ and 11.5 to $15 \mathrm{kHz}$. One range covers the sub-cyclotron frequency range while the other two cover the two obvious features in Figure 86.

An enlargement of the highest frequency band, $10-15 \mathrm{kHz}$ is show in Figure 87. A smoothed version of Figure 87 is shown in Figure 88. The values of the wavenumbers associated with each frequency peak can be identified from the linear wavenumber summation plot shown in Figure 89. The three most prominent peaks occur at $k_{1} \sim$ $0.0089 \mathrm{~cm}^{-1}, k_{2} \sim 0.017 \mathrm{~cm}^{-1}$, and $k_{3} \sim 0.0206 \mathrm{~cm}^{-1}$, all of which represent waves propagating along the background magnetic field towards LEIA.
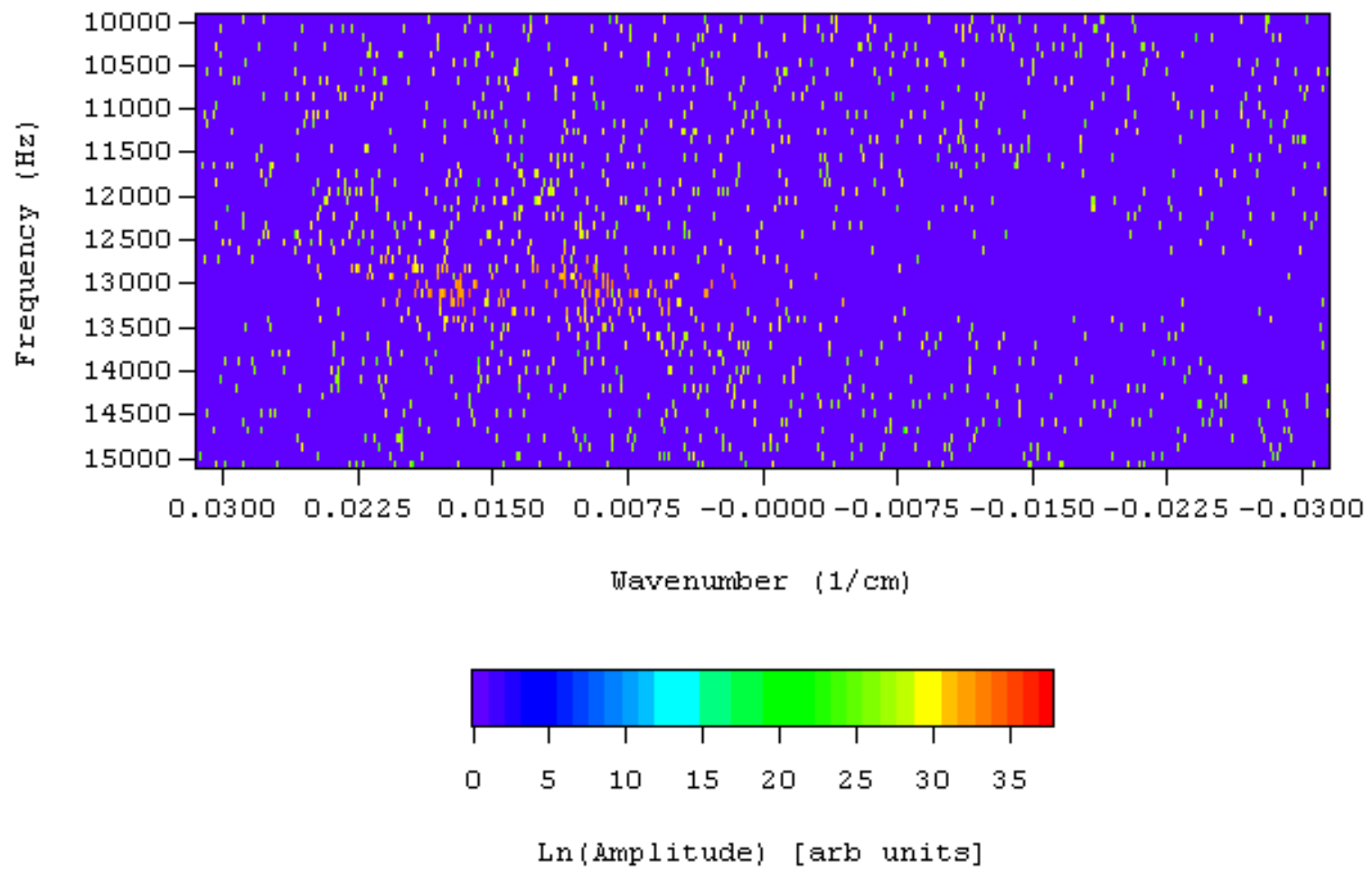

Figure 87: The same data as Figure 86 for the range $10 \mathrm{kHz}-15 \mathrm{kHz}$. 

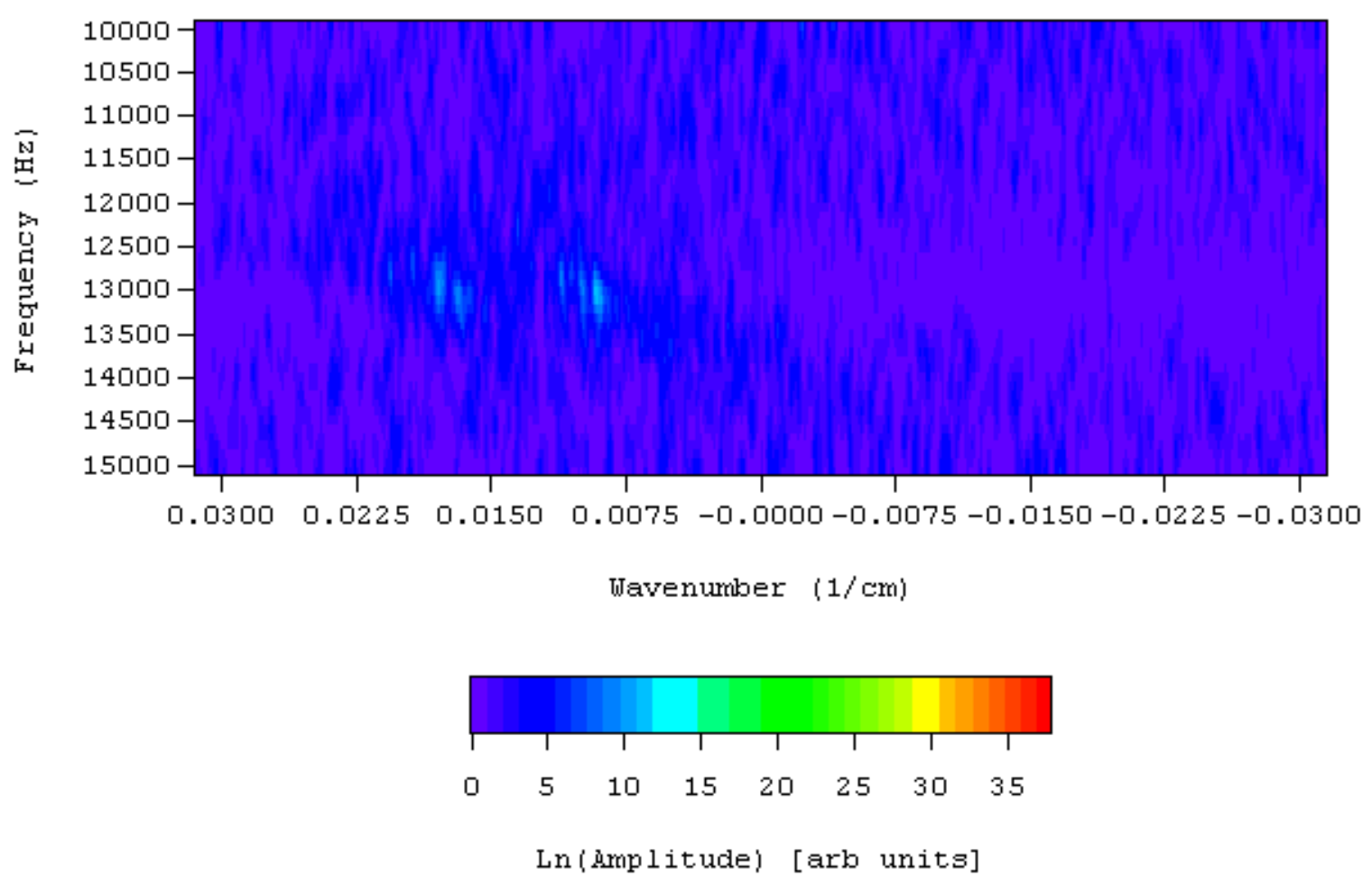

Figure 88: Smoothed version of the data shown in Figure 87. After smoothing, two distinct peaks area clearly visible.

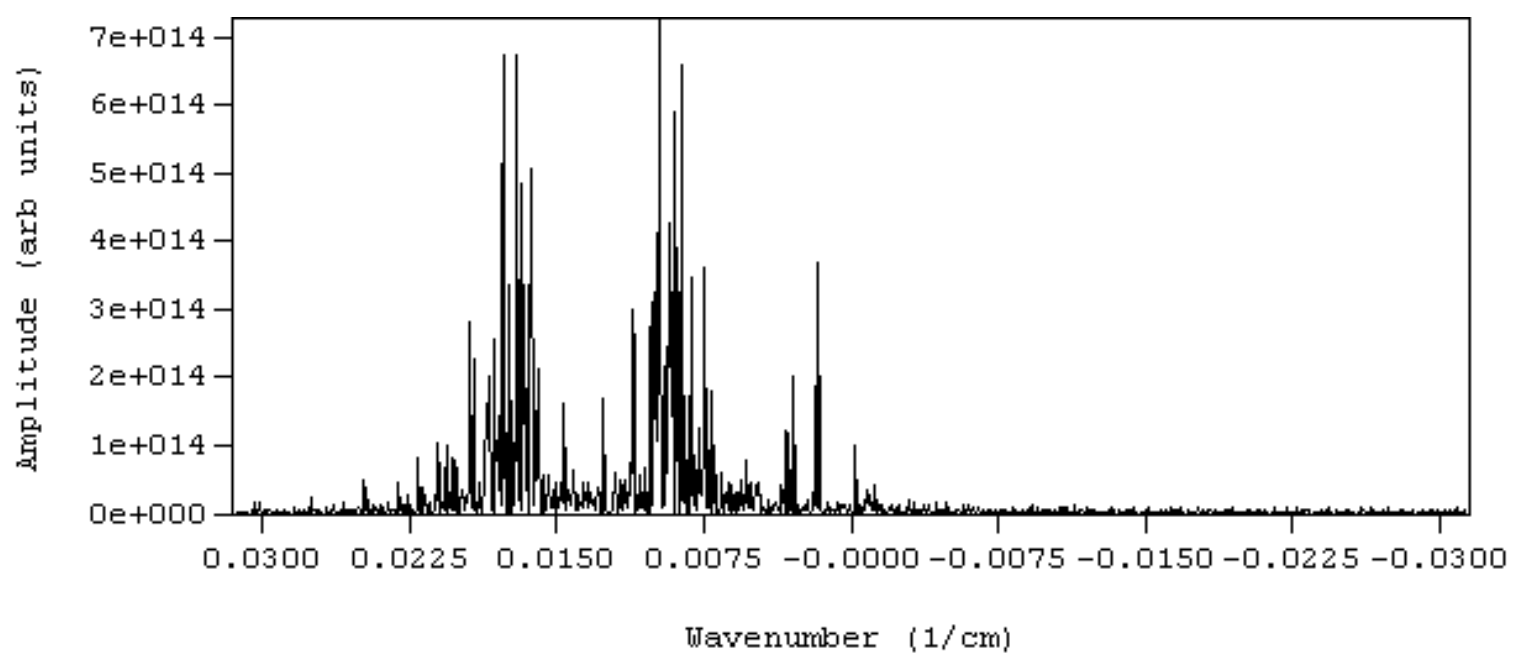

Figure 89: Wavenumber summation of Figure 88 on a linear scale. Three large peaks occur at higher wavenumbers. These are waves propagating towards LEIA. 
Figure 90 is an enlargement of the data in Figure 86 between frequencies of $5 \mathrm{kHz}$ and $8 \mathrm{kHz}$. In the smoothed plot of

Figure 91, two clusters of peaks centered about $6.5 \mathrm{kHz}$ are clearly visible. Unlike the $13 \mathrm{kHz}$ feature, the $6.5 \mathrm{kHz}$ features lie in the negative $k$ region. Figure 92 shows the wavenumber summation for the $5-8 \mathrm{kHz}$ band on a linear scale. Like the $13 \mathrm{kHz}$ feature, this frequency band contains two broad wavenumber peaks and a smaller amplitude, narrow wavenumber peak. However, the $5-8 \mathrm{kHz}$ waves are all propagating upstream towards LEIA. Note the improvement in signal to noise ratio for the $5-8 \mathrm{kHz}$ waves compared to the $10-15 \mathrm{kHz}$ waves.

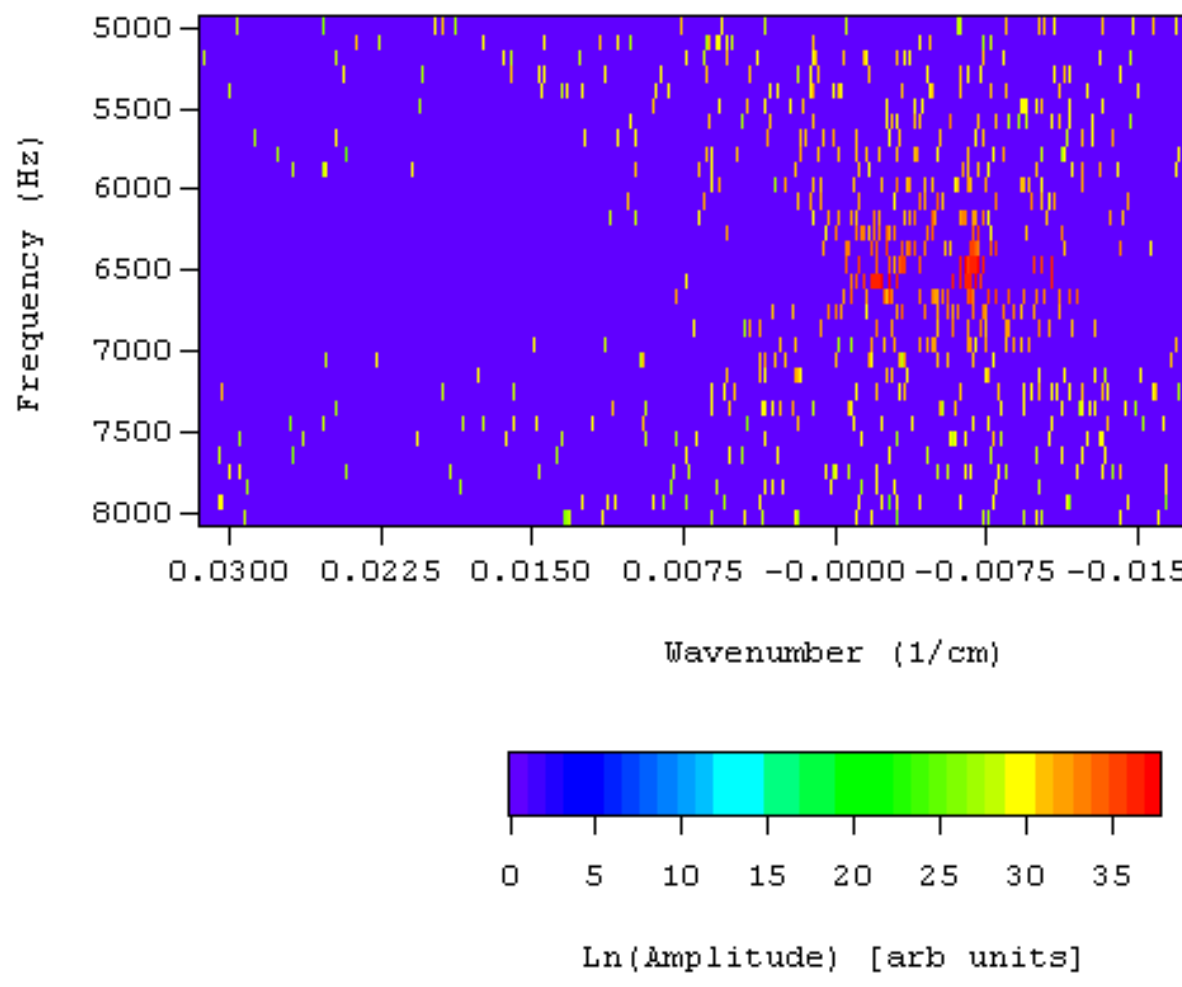

Figure 90: The same data as in Figure 86, but for the frequency range of $5 \mathrm{kHz}$ to $8 \mathrm{kHz}$. 

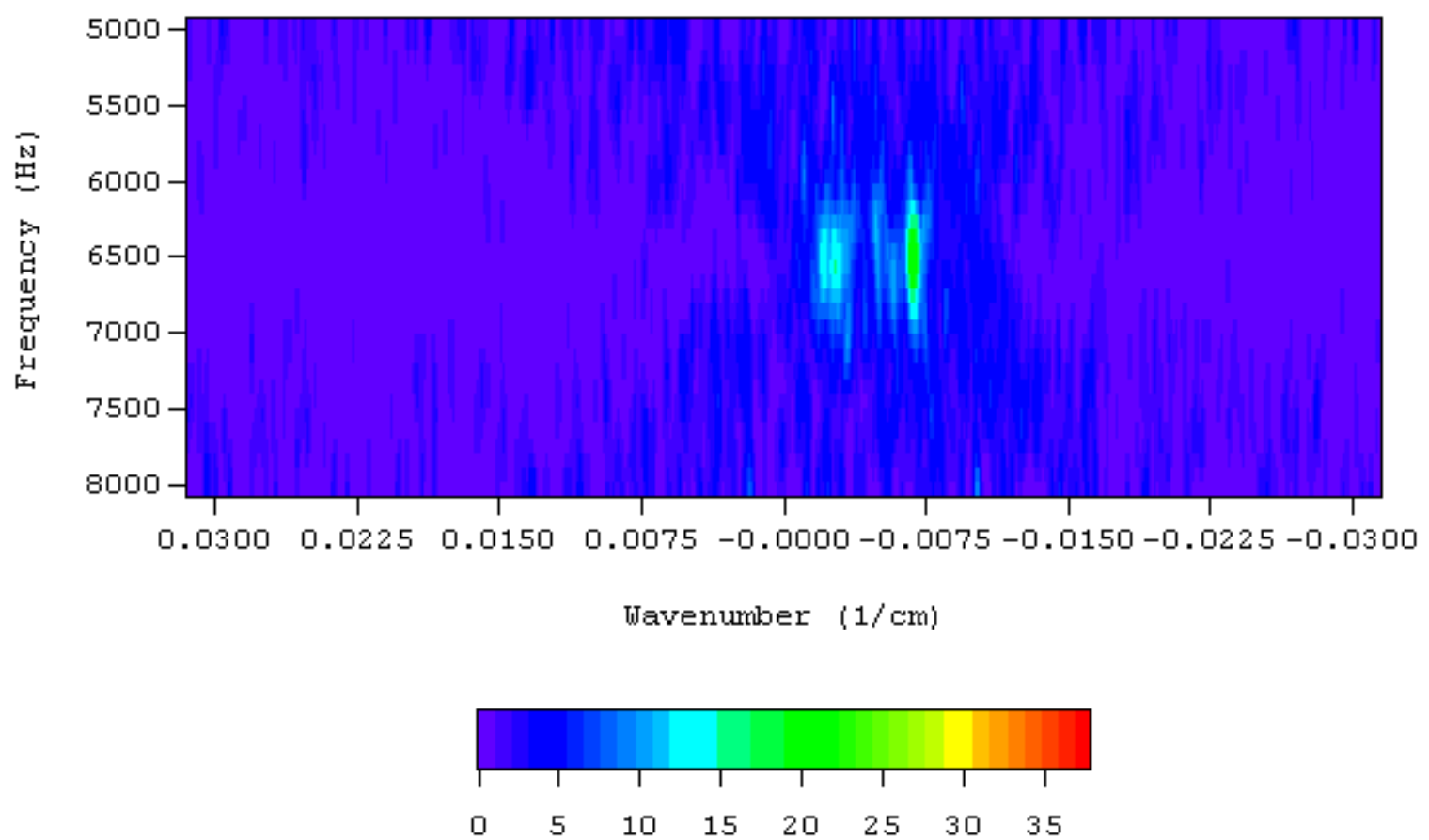

Ln (Ämplitude) [arb units]

Figure 91: Smoothed version of the data shown in Figure 90. After smoothing, two distinct peaks are clearly visible.

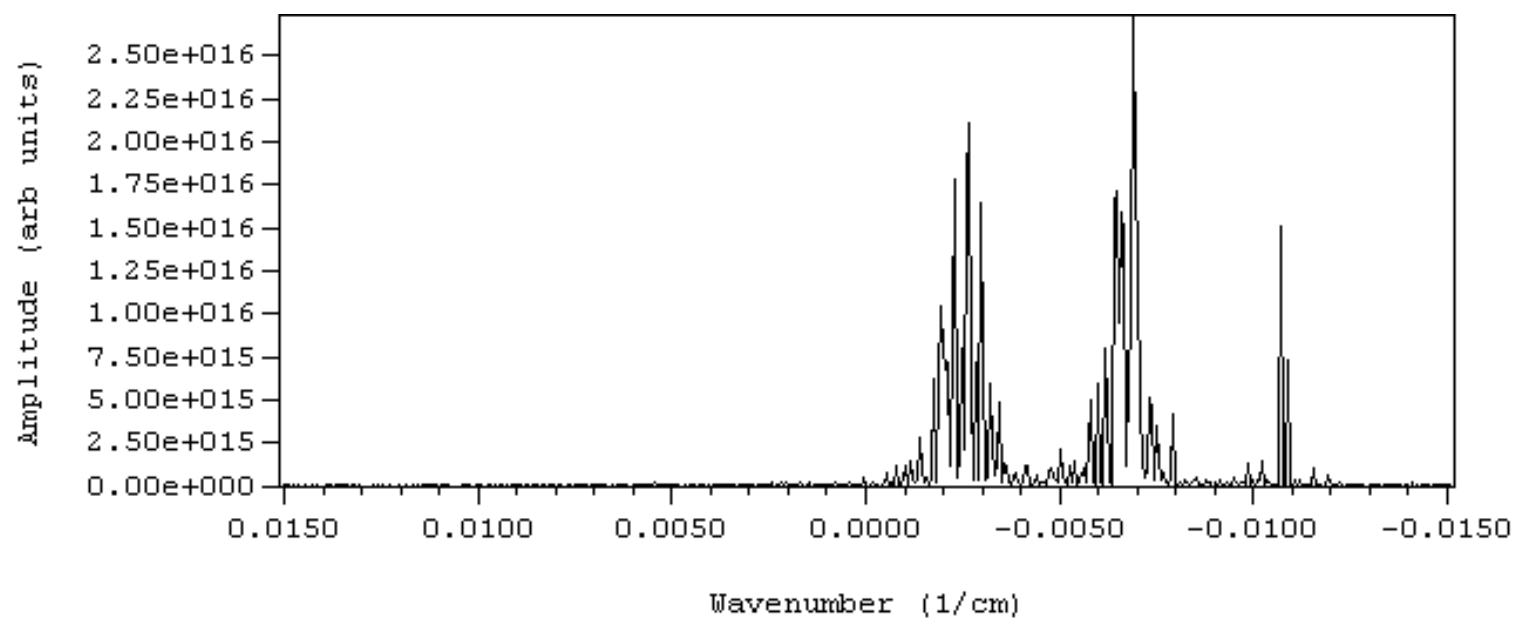

Figure 92: The wavenumber summation over $5-8 \mathrm{kHz}$ for the data of Figure 90.

To determine if ion cyclotron anisotropy instabilities could be identified, the experimental cases for a fixed HELIX magnetic field and different LEIA magnetic fields were examined. Figure 93 shows the low frequency region of the 2D wavenumber 
spectrum for the case of a LEIA magnetic field of $66 \mathrm{G}$, a HELIX field of $556 \mathrm{G}$, and an ion temperature anisotropy of 6.9. Even in the smoothed data shown in Figure 94, it is not possible to visually identify any enhancements at a specific frequency or wavenumber. The wavenumber summation shown in Figure 95 highlights the poor signal to noise ratio at these low frequencies. Fundamentally, the difficulty in measuring wavenumbers at low frequencies is sensitivity. The signal generated by a magnetic sense coil varies linearly with both amplitude and the frequency of the fluctuating wave. The lower the frequency of the wave, the smaller the sense coil signal. Therefore, the best chance of obtaining wavenumber measurements of ion cyclotron anisotropy instabilities should occur at the highest LEIA fields possible (to raise the gyrofrequency to a region of better sensitivity) and the highest ion temperature anisotropy possible (to increase the wave amplitude).
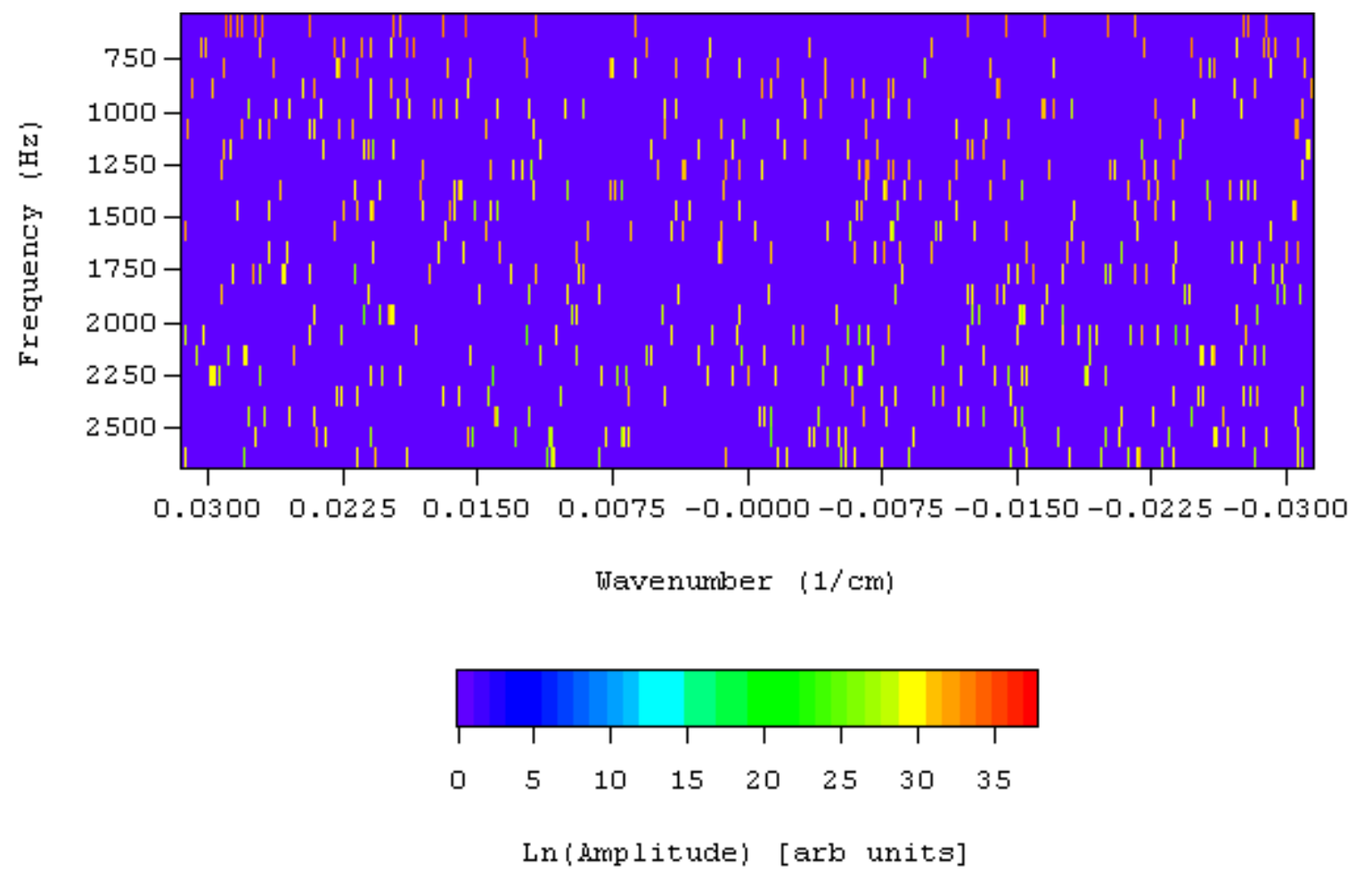

Figure 93: Enlargement of the frequency range of 0.6 to $2.6 \mathrm{kHz}$ for the same data shown in Figure 86. 

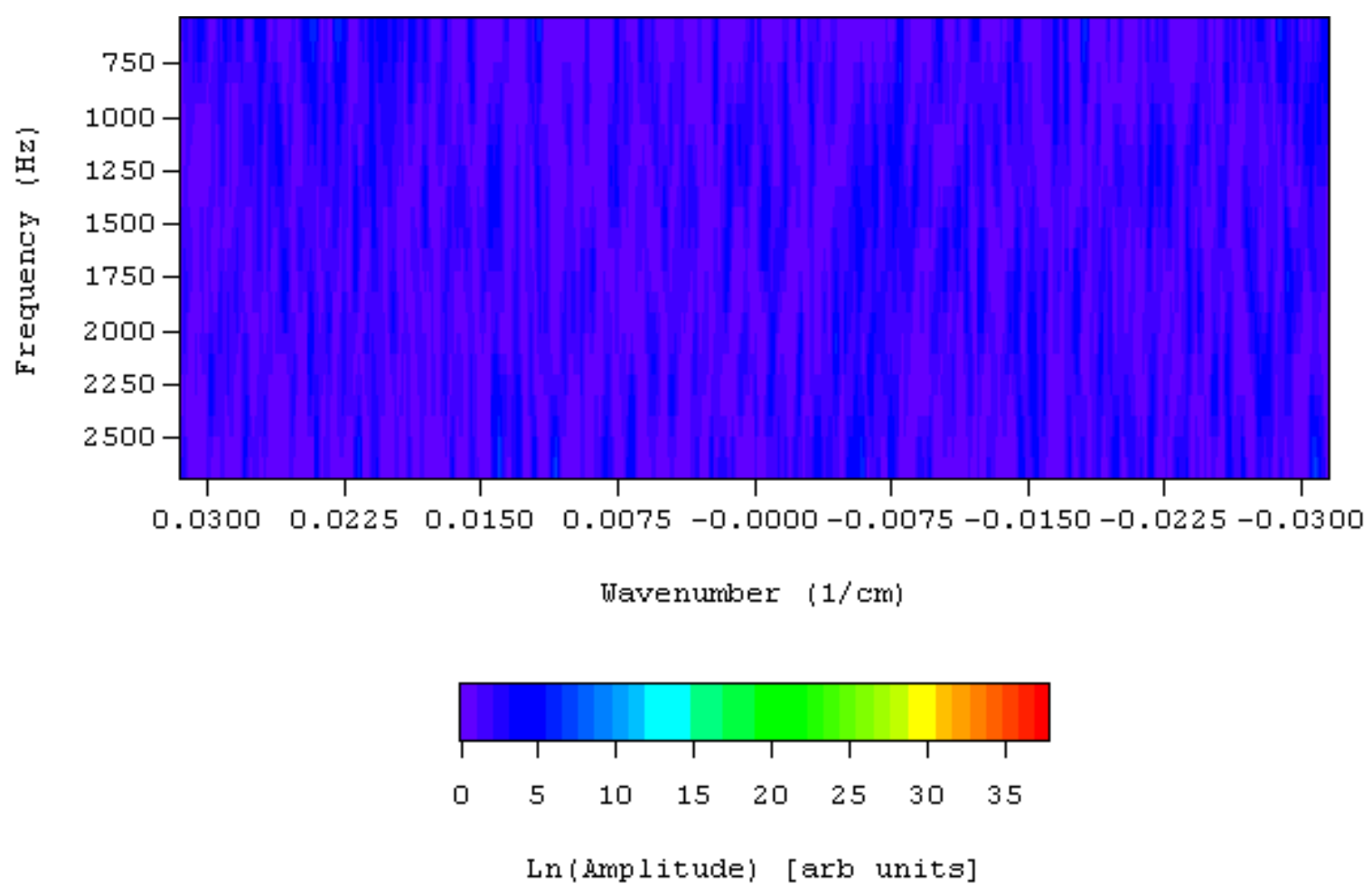

Figure 94: Same data as Figure 93 after smoothing. There are no distinct spectral features.

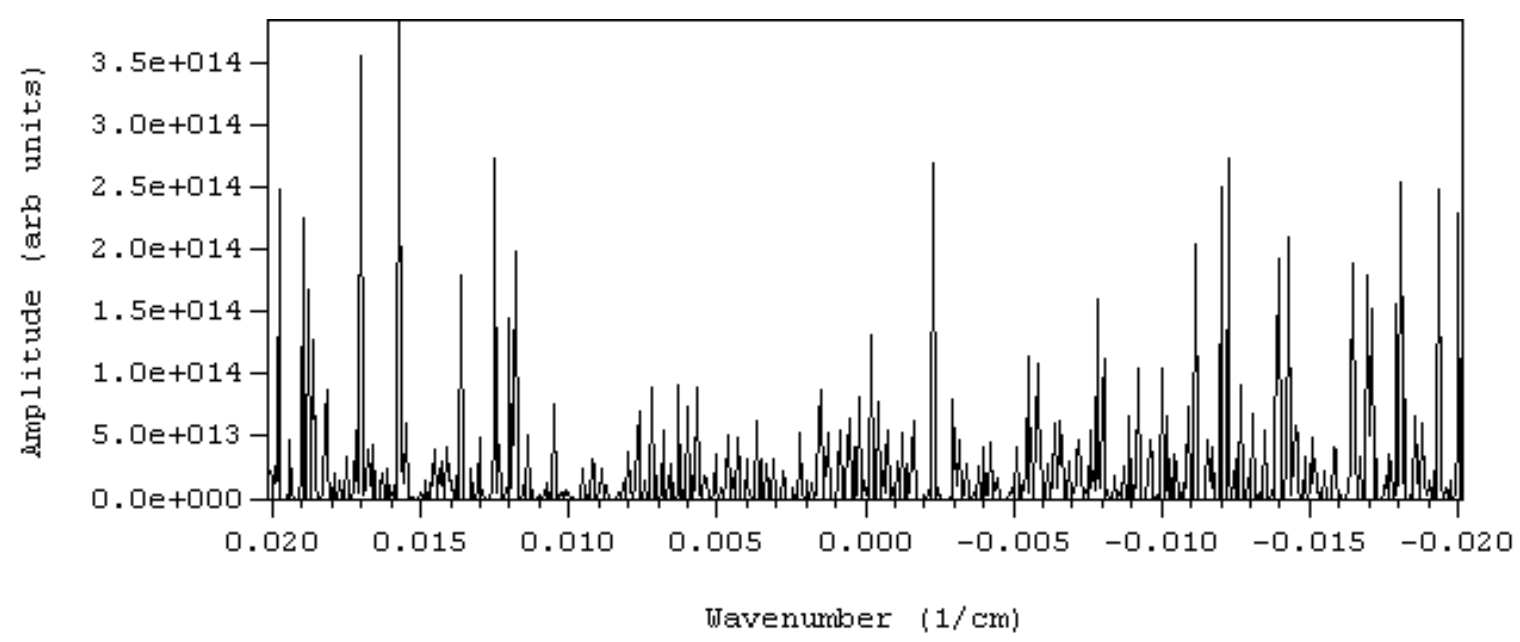

Figure 95: The wavenumber summation over .6 to $2.6 \mathrm{kHz}$ for the data of Figure 93.

Figure 96 shows the normalized wavenumber summation from $1-2.7 \mathrm{kHz}$ for a HELIX field of $1264 \mathrm{G}$, a LEIA field of $70 \mathrm{G}$, and an ion temperature anisotropy of 8.3. 
The largest wavenumber peaks occur in the normalized wavenumber range of $0.5-1.5$. For reference, Figure 43 and Figure 45 display the theoretically predicted normalized wavenumber, $k c / \omega_{p i}$ values for the proton cyclotron anisotropy instability. Although those graphs are for somewhat different parameters $\left(\beta\right.$ and $\left.T_{e}\right)$ than the parameters of these experiments, they provide rough estimates of the allowed $k$ value range. The wavenumber spectrum shown in Figure 96 is consistent with the theoretical prediction of Figure $45, k_{\text {norm }} \sim 1$, and also consistent with waves traveling from HELIX to LEIA.

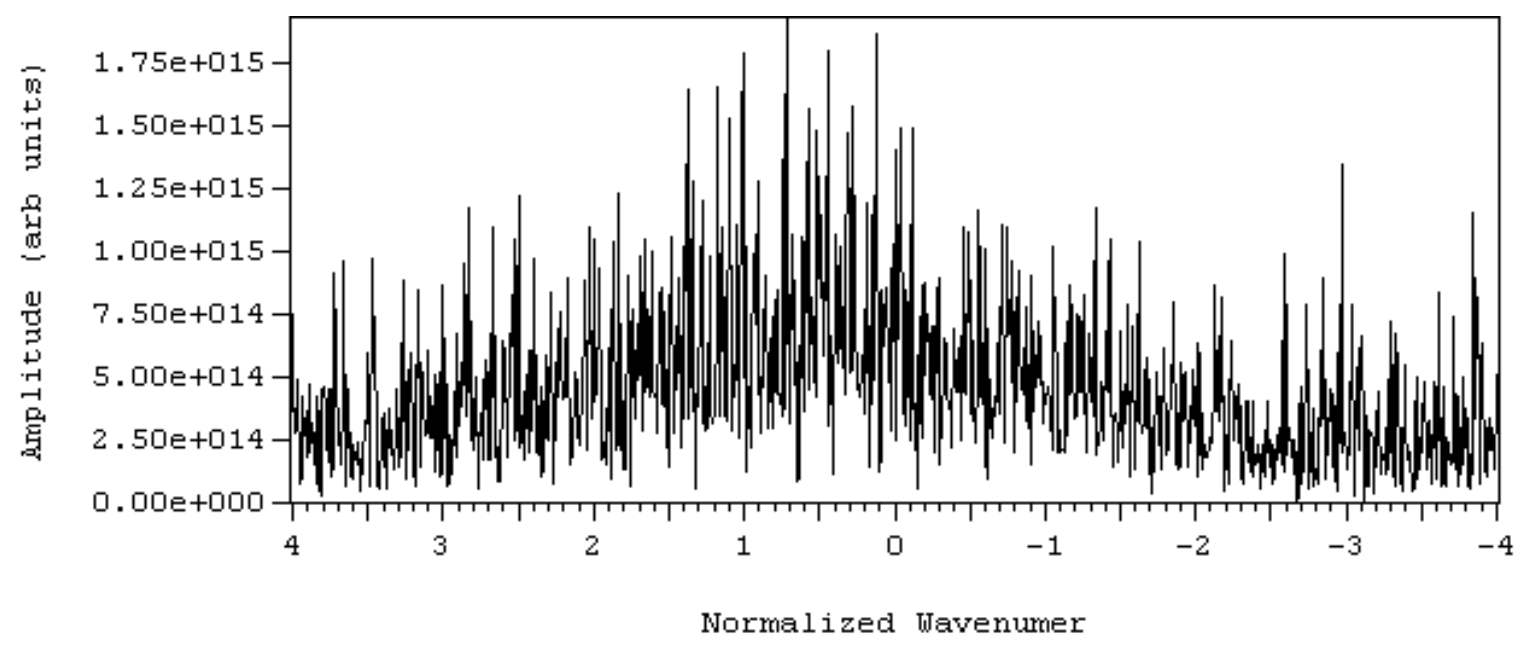

Figure 96: Wavenumber summation between 1 - $2.7 \mathrm{kHz}$ on a linear scale for a HELIX magnetic field of $1264 \mathrm{G}$, a LEIA magnetic field of $70 \mathrm{G}$, and an ion temperature anisotropy of 8.3. The features around a normalized wavenumber of unity are consistent with theory. ${ }^{91}$

Figure 97 shows the normalized wavenumber spectrum from $1-2.7 \mathrm{kHz}$ for a HELIX magnetic field of $1028 \mathrm{G}$, a LEIA magnetic field of $68 \mathrm{G}$, and an ion temperature anisotropy of 4.1. Other than a slight enhancement and large positive $k$, there are no distinct features in the data. 


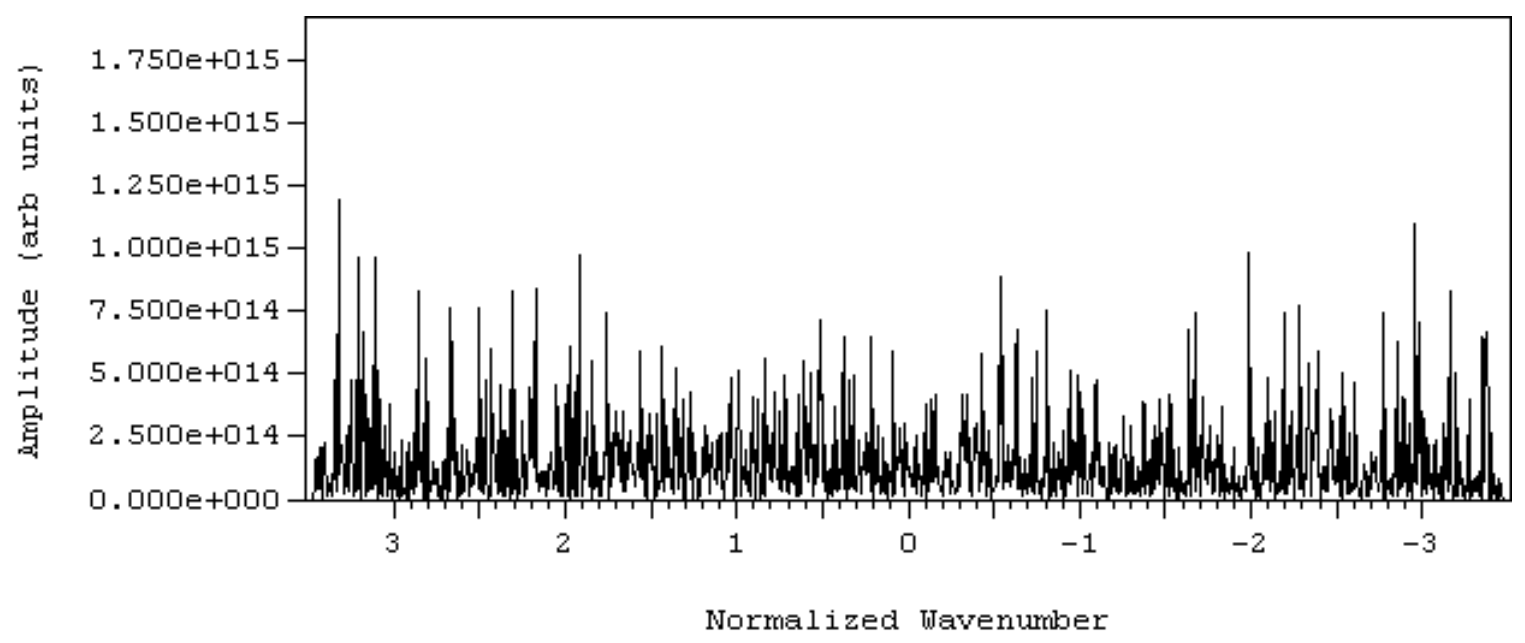

Figure 97: Wavenumber summation between 1 - $2.7 \mathrm{kHz}$ on a linear scale for a HELIX magnetic field of $1028 \mathrm{G}$, a LEIA magnetic field of $38 \mathrm{G}$, and an ion temperature anisotropy of 4.1 .

Figure 98 shows the wavenumber summation from $1-2.7 \mathrm{kHz}$ for a HELIX magnetic field of $792 \mathrm{G}$, a LEIA magnetic field of $68 \mathrm{G}$, and an ion temperature anisotropy of 7.3. This case does not have as broad of a range of wavenumbers as Figure 96, but the positive $k$ portion of the spectrum is clearly enhanced. The three largest peaks occur at normalized wavenumbers of roughly $0.6,1.4$, and 2.5 . The $0.6-1.4$ peaks are consistent with the theory predictions of Figure 45.

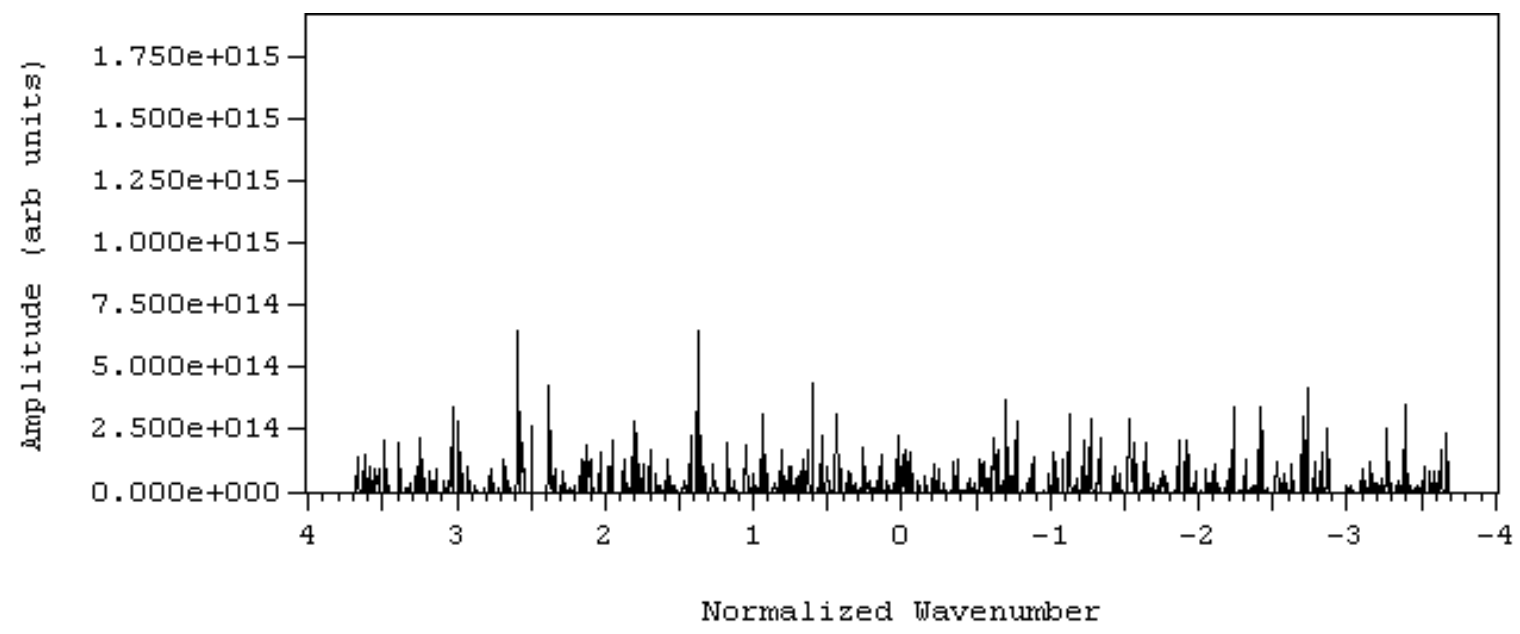

Figure 98: Wavenumber summation between $1-2.7 \mathrm{kHz}$ on a linear scale for a HELIX magnetic field of 792, a LEIA magnetic field of $68 \mathrm{G}$ and an anisotropy of 7.3. 
Figure 99 shows the wavenumber summation from 1- $2.5 \mathrm{kHz}$ for a HELIX magnetic field of $556 \mathrm{G}$, a LEIA magnetic field of $66 \mathrm{G}$, and an ion temperature anisotropy of 6.9 . Here again, the signal to noise ratio is too low to draw any conclusions.

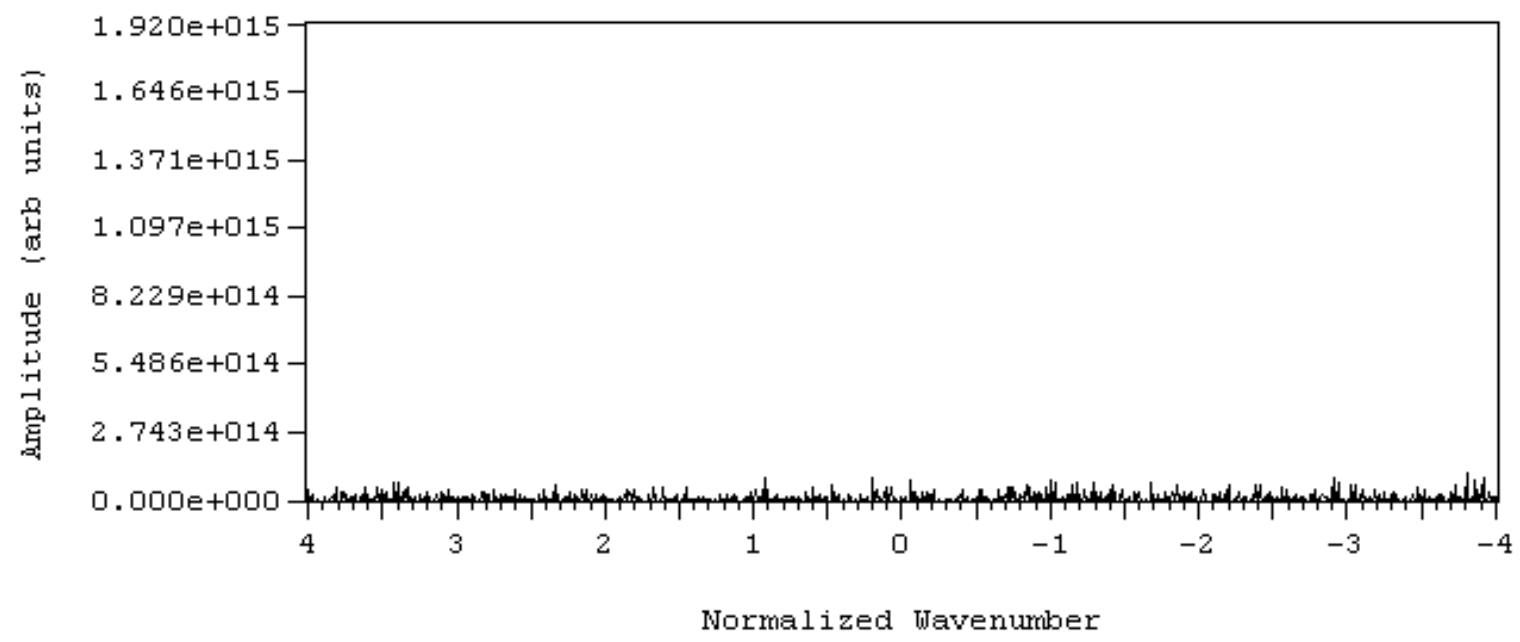

Figure 99: Wavenumber summation between $1-2.5 \mathrm{kHz}$ on a linear scale for a HELIX magnetic field of $556 \mathrm{G}$, a LEIA magnetic field of $66 \mathrm{G}$ and an anisotropy of 6.9.

As expected, the cases with the highest ion cyclotron frequencies and the largest ion temperature anisotropies in LEIA have the best signal to noise levels in the wavenumber data. In general, the wavenumber peaks are consistent with the predictions for ion cyclotron anisotropy instabilities. However, the overall poor signal-to-noise prohibits the interpretation that these wavenumber measurements offer conclusive evidence that the low frequency waves are ion cyclotron anisotropy instabilities. 


\section{DISCUSSION}

These experiments demonstrate the existence of an inverse correlation between the ion temperature anisotropy and the parallel ion beta in a high beta laboratory plasma. The scaling of the correlation is in good agreement with both theoretical models and spacecraft observations for ion temperature anisotropies limited by the action of electromagnetic ion temperature anisotropy instabilities. The apparent universality of the inverse correlation between ion temperature anisotropy and parallel ion beta supports Manheimer and Boris' claim that a plasma instability threshold derived from linear theory should correspond to an observable bound on the ion temperature anisotropy.

To reduce the possible effect of varying the collisionality in interpreting this marginally collisional laboratory experiment, a subset of data with roughly the same ionneutral collision and ion-ion thermalization rates was identified. Within that data subset, the inverse correlation was still observed. The effect of low frequency waves on the reduction of anisotropy between upstream and downstream positions was studied in a controlled experiment. The amount of anisotropy reduction decreased when the amplitudes of low frequency electromagnetic waves decreased. This suggests that the low frequency waves are related to the anisotropy reduction observed in LEIA. Magnetic fluctuation spectra of the low frequency waves demonstrated an exponential scaling of the low frequency electromagnetic wave power with ion beta. The exponential nature of the wave power scaling is in contrast to the power law scaling predicted by theory. The electromagnetic wave measurements clearly indicate that the low frequency waves are transverse, not compressional. In the experimental cases with the highest cyclotron frequencies and the highest ion temperature anisotropies, the observed wavenumbers are consistent with the wavenumber values predicted for ion cyclotron anisotropy instabilities. Although these low frequency fluctuations can not be conclusively identified as ion cyclotron anisotropy waves because of poor signal to noise ratio in the wavenumber measurements, the observed characteristics of these waves suggest the waves are most consistent with ion cyclotron anisotropy instabilities. 
To enable better identification of low frequency waves in LEIA, there are a number of experimental modifications that could be implemented. Fundamentally, the signal-tonoise ratio below $3 \mathrm{kHz}$ can be improved. One way of accomplishing this is by increasing the number of turns on the magnetic sense coils, which will increase the sensitivity at lower frequencies. Taking more realizations than the fifty used in these studies will also improve the signal-to-noise ratio in the wavenumber analysis. Reducing the sampling rate, would improve frequency space resolution and accentuate features in the power spectra of the low frequency waves. With better signal-to-noise, the polarization of the low frequency waves could be determined with hodograms constructed from $B_{r}$ and $B_{\theta}$ data. As the wavenumber measurements presented here indicate the wavelengths in LEIA are on the order of a few meters, wavenumber measurements with larger probe separations would provide improved phase difference sensitivity.

Detailed electrostatic probe measurements over the parameter range used in these experiments will play a key role in verifying that the low frequency waves are indeed primarily electromagnetic. Development of LIF techniques to identify electromagnetic waves through perturbations of the ion distribution function could also be used in conjunction with magnetic sense coils observations to better identify low frequency waves. 


\section{REFERENCES}

${ }^{1}$ Manheimer, W. and J. P. Boris, "Marginal stability analysis - A simpler approach to anomalous transport in plasmas," Comments Plasma Phys. Controlled Fusion, 3, 15 (1977).

${ }^{2}$ Gary, S. P., J. Wang, D. Winske, and S. A. Fuselier, "Proton temperature anisotropy upper bound," J. Geophys. Res., 102, 27159 (1997).

${ }^{3}$ Gary, S. Peter, Michael E. McKean, Dan Winske, Brian J. Anderson, Richard E. Denton and Stephen A. Fusilier, "The proton cyclotron instability and the anisotropy/ $\beta$ inverse correlation," J. Geophys. Res., 99, 5903 (1994).

${ }^{4}$ Anderson, Brian J., Stephan A. Fuselier, S. Peter Gary, and Richard E. Denton, "Magnetic spectral signatures in the Earth's magnetosheath and plasma depletion layer," 99, J. Geophys. Res. 5877 (1994).

${ }^{5}$ Phan, T.-D., G. Paschmann, W. Baumjohann, N. Sckopke, and H. Luhr, "The magnetosheath region adjacent to the dayside magnetopause: AMPTE/IRM observations," J. Geophys. Res., 99, 121 (1994).

${ }^{6}$ Fuselier, Stephan A., Brian J. Anderson, S. Peter Gary, and Richard E. Denton, "Inverse correlations between the ion temperature anisotropy and plasma beta in the Earth's quasi-parallel magnetosheath," J. Geophys. Res., 99, 14,931 (1994).

${ }^{7}$ Scharer, J. E., and A. W. Trivelpiece, "Cyclotron wave instabilities in a plasma," Phys. Fluids, 10, 591 (1967).

${ }^{8}$ Nakamura, Motoyuki et al., "Excitation of the Alfvén ion cyclotron mode soley by the ion pressure distribution," Jpn. J. Appl. Phys., 37, 342 (1998).

${ }^{9}$ Casper, T. A., and Gary R. Smith, "Observation of Alfvén Ion-CyclotronFluctuations in the End-Cell Plasma in the Tandem Mirror Experiment," 48, 1015 (1982).

${ }^{10}$ Kivelson, Margaret G., and Christopher T. Russell, "Introduction to Space Physics," Cambridge University Press (1995).

${ }^{11}$ Hill, K. W, et al., "Tests of local transport theory and reduced wall impurity influx with highly radiative plasmas in the Tokamak Fusion Test Reactor," Phys. Plasmas, 6, 877 (1999).

${ }^{12}$ Greenwald, et al., "Characterization of enhanced $\mathrm{D}_{\alpha}$ high-confinement modes in Alcator C-Mod", Phys. Plasmas, 6, 1943 (1999).

${ }^{13}$ Chen, Francis F., "Introduction to plasma physics and controlled fusion," Plenum Press (1984).

${ }^{14}$ Chevalier, G, and F. F. Chen, "Experimental modeling of inductive discharges", J. Vac. Sci. Technol. A., 11, 1165 (1993).

${ }^{15}$ Chen, Francis F., "Capacitor tuning circuits for inductive loads", UCLA Internal Report PPG-1401, 1992.

${ }^{16}$ Balkey, M, " Investigation of different antenna designs on HELIX," WVU Internal Report (1999).

${ }^{17}$ Kline, John, E. E. Scime, P. A. Keiter, M. M. Balkey, R. F. Boivin, "Ion heating in the HELIX helicon plasma source," submitted to Phys. Plasmas, (1999).

${ }^{18}$ Light, Max, Isaac D. Sudit, Francis F. Chen, and Donald Arnush, "Axial propagation of helicon waves," Phys. Plasmas, 2, 4094 (1995).

${ }^{19}$ Guo, X. M., J. Scharer, Y. Mouzouris, and L. Louis, "Helicon experiments and simulations in nonuniform magnetic field configurations," University of Wisconsin Internal Report, UW-CPTC 98-14, (1998).

${ }^{20}$ Shoji, T, Y. Sakawa, S. Nakazawa, K. Kadota, and T. Sato, "Plasma production by helicon waves," Plasma Sources Sci. Technol., 2, 5 (1993).

${ }^{21}$ Boswell, R.W., "Plasma Production using a standing helicon wave," Physics Letters, 33A, 457 (1970).

${ }^{22}$ Perry, A.J., D. Vender, and R. W. Boswell, "The application of the helicon source to plasma processing," J. Vac. Sci. Technol. B, 9, 310 (1991).

${ }^{23}$ Jiwari, Nobuhiro, Hiroaki Isasawa, Akira Narai, Hiroyuki Sakaue, Haruo Shindo, Tatsuo Shoji and Yasuhiro Horiike, "Al etching characteristics employing helicon wave plasma," Jpn. J. Appl. Phys., 32, 3019 (1993). 
${ }^{24}$ Katyukha, V. P., G. S. Kirichenko, A. V. Rusavskii, V. B. Taranov and K. P. Shamrai, "Helicon ion source for plasma processing," Rev. Sci. Instrum., 65, 1368 (1994).

${ }^{25}$ Mieno, T., T. Kamo, D. Hayashi, T. Shoji and K. Kadota, "Efficient production of $\mathrm{O}^{+}$and $\mathrm{O}^{-}$in a helicon wave oxygen discharge," Appl. Phys. Lett., 69, 617 (1996).

26 Tsukada, Tsutomu, Hiroshi Nogami, Jun Hayashi, Kazu Kawaguchi and Toru Hara, "Physical damage in silicon formed by helicon wave plasma etching," J. Appl. Phys., 74, 5402 (1993).

${ }^{27}$ Shats, M. G., D. L. Rudakov, R. W. Boswell, and G. G. Borg, "Ion temperature and plasma flows in improved confinement mode in the H-1 heliac," Phys. Plasmas, 4, 3629 (1997).

${ }^{28}$ Chang-Diaz, F. R., R. H. Goulding, R. D. Bengtson, F. Wally-Baity, D. Sparks, R. G. Russel Jr., G. C. Barber, G. McCaskill, V. T. Jacobson, M. D. Carter, A. V. Ilin, T. W. Glover, "Experimental status of the development of a variable specific impulse magnetoplasma rocket," Fusion Technology, 35, 243 (1999).

${ }^{29}$ Aigrain, P., "Les 'helicons' dans les semiconducteurs," Proc. Int. Conf. Seniconductor Physics, 224, (1960).

${ }^{30}$ Legendy, C. R., "Macroscopic theory of helicons," Phys. Rev., 135, 1713 (1965).

${ }^{31}$ Klosenberg, J. P., B. McNamara and P. C. Thoneman, "The dispersion and attenuation of helicon waves in a uniform cylindrical plasma," J. Fluid Mech., 21, 545 (1965).

${ }^{32}$ Boswell, R. W., "Modulated rf produced argon magneto-plasma," The Flinders University of South Australia Internal Rep., PR 68/8, 1968.

${ }^{33}$ Boswell, Rod W., and Francis F. Chen, "Helicons - The early years," IEEE Transactions on Plasma Science, 25, 1229 (1997).

${ }^{34} \mathrm{Zhu}$, Peiyuan and R. W. Boswell, "Observation of nonthermal electron tails in an rf exited argon magnetoplasma," Phys. Fluids B, 3, 869 (1991).

${ }^{35}$ Boswell, R.W., "Very efficient plasma generation by whistler waves near the lower hybrid frequency," Plasma Phys. Control. Fusion, 26, 1147 (1984).

${ }^{36}$ Ellingboe, A. R., and R. W. Boswell, "Capacitive, inductive and helicon-wave modes of operation of a helicon plasma source," Phys. Plasmas, 3, 2797 (1996).

${ }^{37}$ Keiter, Paul A., Earl E. Scime, and Matthew M. Balkey, "Frequency dependent effects in helicon plasmas," Phys. Plasmas, 4, 2741 (1997).

${ }^{38}$ Shoji, T., IPPJ Annu. Rev., Nagoya Univ., Japan, 67 (1986).

${ }^{39}$ Suddit, Isaac D., and Francis F. Chen, "Discharge equilibrium of a helicon discharge," Plasma Sources Sci. Technol., 5, 43 (1995).

${ }^{40}$ Guo, X. M., J. Scharer, Y. Mouzouris and L. Louis, "Helicon experiments and simulations in nonuniform magnetic field configurations," Phys. Plasmas, 6, 3400 (1999).

${ }^{41}$ Klinger, Thomas, "Control of plasma instabilities in laboratory plasmas," IPELS meeting, 1999.

${ }^{42}$ Watts, Christopher, "Laboratory Alfvén wave measurements in a steady state, high-density plasma," P.3.10, IPELS meeting, 1999.

${ }^{43}$ Stix, T. H., "Waves in Plasmas" (American Institute of Physics, New York, 1992).

${ }^{44}$ Borg, G. G. and R. W. Boswell, " Power coupling to helicon and Trivelpiece-Gould modes in helicon sources," Phys. Plasmas, 5, 564 (1998).

${ }^{45}$ Chen, Francis F., "Plasma ionization by helicon waves," Plasma Phys. And Contr. Fus., 33, 339 (1991).

${ }^{46}$ Yun, Seok-Min, Jung-Hyung Kim and Hong-Young Chang, "Frequency dependence of helicon wave plasmas near lower hybrid resonance frequency," J. Vac. Sci. Technol. A., 15, 673 (1997).

${ }^{47}$ Komori, A., T. Shoji, K. Miyamoto, J. Kawai, Y. Kawai, "Helicon waves and efficient plasma production," Phys. Fluids B, 3, 893 (1991).

${ }^{48}$ Chen, Francis, F., Hsieh, M. Johannes, and Max Light, "Helicon waves in a non-uniform plasma," Plasma Sources Sci. Technol., 3, 49 (1994).

${ }^{49}$ Chen, Francis F., "Experiments on helicon plasma sources," J. Vac. Sci. Technol. A, 10, 1389 (1992).

50 Jackson, J., "Classical Electrodynamics," John Wiley \& Sons Inc., New York, (1975)

${ }^{51}$ Chen, Francis F., and Christopher D. Decker, "Electron Acceleration in Helicon Sources," Plasma Physics and Controlled Fusion, 34, 635 (1992). 
${ }^{52}$ Molvik, A.W., A. R. Ellingboe and T. D. Rognmien, "Hot-Electron Production and Wave Structure in a Helicon Plasma Source," Physical Review Letters, 79, 233 (1997).

${ }^{53}$ Chen, Francis F. and David D. Blackwell, "Upper limit to Landau damping in helicon discharges," UCLA Internal Report LTP-809, 1998.

${ }^{54}$ Degeling, A. W., C. O. Jung, R. W. Boswell, and A. R. Ellingboe, "Plasma production from helicon waves," 3, 2788 (1996)

${ }^{55}$ Chen, F. F., X. Jiang, J. D. Evans, G. Tynan, and D. Arnush, "Low field helicon discharges," UCLA Internal Report PPG-1564 (1996).

${ }^{56}$ Chen, F. F., and G. Chevalier, "Experiments on helicon plasma sources," J. Vac. Sci. Technol. A, 10, 1389 (1992).

${ }^{57}$ Davies, B., "Helicon wave propagation: effect of electron inertia," J. Plasma Physics, 4, 43 (1970).

${ }^{58}$ Boswell, R. W., "Dependence of helicon wave radial structure on electron inertia," Aust. J. Phys., 25, 403 (1972).

${ }^{59}$ Shamrai, K. P., and V. B. Taranov, "Resonance wave discharge and collisional energy absorption in helicon plasma source," Plasma Phys. Control. Fusion, 36, 1719 (1994).

${ }^{60}$ Chen, Francis F. and Rod W. Boswell, "Helicons - The past decade," IEEE Transactions on Plasma Science, 25, 1245 (1997).

${ }^{61}$ Hutchinson, I. H., "Principles of Plasma Diagnostics," Cambridge University Press, 1987.

${ }^{62}$ Krall, Nicholas A., and Alvin W. Trivelpiece, "Principles of plasma physics," San Francisco Press, 1986.

${ }^{63}$ Boschi, A. and Magistrelli, F., Nuovo Cimento, 29, 487 (1962).

${ }^{64}$ Hershkowitz, N., M. H. Cho, C. H. Nam, and T. Intrator, " Langmuir probe characteristics in RF glow discharges ," Plasma Chem. And Plasma Proc. 8, 35 (1988).

${ }^{65}$ Sudit, Isaac D. and Francis F. Chen, "RF compensated probes for high-density discharges," Plasma Sources Sci. Techol., 3, 162 (1994).

${ }^{66}$ Godyak, V. A., R. B. Piejak, and B. M. Alexandrovich, " Measurement of electron energy distribution in low-pressure RF discharges" Plasma Sources Sci. Technol., 1, 36 (1992).

${ }^{67}$ Measures, R. M., "Selectrive excitation spectroscopy and some possible apllications," J. Appli. Phys., 39, $5232(1968)$.

${ }^{68}$ Skiff, F., and F. Anderegg, " Direct observation of plasma dielectric motion ," Phys. Rev. Lett. 59, 896 (1987).

${ }^{69}$ Sarfaty, M., S. De Souza-Machado, and F. Skiff, " Direct determination of ion wave fields in a hot magnetized and weakly collisional plasma," Phys. Plasmas 3, 4316 (1996).

${ }^{70}$ McChesney, Jon Mearns, "Observation of stochastic ion heating by low frequency drift waves," Ph.D. Thesis (1989).

${ }^{71}$ Moore, C. A., G. P. Davis and R. A. Gottscho, "Sensitive, nonintrusive in-situ measurement of temporally and spatially resolved plasma electric fields," Phys. Rev. Lett., 52, 538 (1984).

${ }^{72}$ West, W. P., D. M. Thomas. E. S. Ensberg, J. S. DeGrassie, and J. F. Baur, "Diagnostic instrument for the measurement of poloidal magnetic fields in tokamaks," Rev. Sci. Instr., 57, 1552 (1986).

${ }^{73}$ Boivin, R. F., "Study of the different line broadening mechanisms for the laser induced fluorescence diagnositic for the HELIX and LEIA plasmas," WVU Internal Report PL-039, (1998).

${ }^{74}$ Wise, W. L., "Line Broadening," Plasma Diagnostic techniques ed. By Richard H. Huddlestone and Stanley L. Leonard, 269 (1965).

${ }^{75}$ Griem, H. R., "Plasma Spectroscopy," McGraw-Hill, New York, (1964).

${ }^{76}$ Demtroder, W., "Laser Spectroscopy," Spinger Verlag (1981).

${ }^{77}$ Goeckner M. J., and J. Goree, "Laser-induced fluorescence measurement of plasma ion temperatures: corrections for power saturation," J. Vac. Sci. Technol. A, 7, 977 (1989).

${ }^{78}$ J. M. Beall, Y. C. Kim, and E.J. Powers, "Estimation of wavenumber and frequency spectra using fixed probe pairs," J. Appl. Phys., 53, 3993 (1982).

${ }^{79}$ Bendat, Julius S., and Allan G. Piersol, "Engineering applications of correlation and spectral analysis," John Wiley \& Sons Inc., (1980).

${ }^{80}$ Hundhausen, A. J., J. R. Asbridge, S. J. Bame, H. E. Gilbert and I. B. Strong, "Vela 3 satellite observations of solar wind ions: A priliminary report," J. Geophys. Res., 72, 87 (1967). 
${ }^{81}$ Wolfe, J. H., R. W. Silva, D. D. McKibbin and R. H. Mason, "The compositional, anisotropic and nonradial-flow characteristics of the solar wind," J. Geophys. Res., 71, 3329 (1966).

${ }^{82}$ Scarf, F. L., J. H. Wolfe, and R. W. Silva, "A plasma instability associated with thermal anisotropies in the solar wind," J. Geophys. Res., 72, 993 (1967).

${ }^{83}$ Holzer, R. E., M. G. McLeod and E. J. Smith, "Preliminary results from the OGO 1 search coil magnetometer; Boundary positions and magnetic noise spectra," J. Geophys. Res., 71, 1481 (1966).

${ }^{84}$ Coleman, P. J., Jr. "Variations in the interplanetary magnetic field: Mariner 2, 1, Observed properties," J. geophys. Res., 71, 5509 (1966).

${ }^{85}$ Siscoe, G. L., L. Davies Jr., P. J. Coleman Jr., E. J. Smith and D. E. Jones, "Power Spectra and discontinuities of the interplanetary magnetic field: Mariner 4,"J. Geophys. Res., 73, 61 (1968).

${ }^{86}$ Ness, N. F., G. S. Scearce and S. Cantarano, "Preliminary results from the Pioneer 6 magnetic field exponent," J. Geophys. Res., 71, 3305 (1966).

${ }^{87}$ Kennel, C. F. and H. E. Petschek, "Limit on stabley trapped particle fluxes," J. Geophys. Res., 71, 1 (1966).

${ }^{88}$ Chandrasekhar, S., A. N. Kaufman, and K. M. Watson, "The stability of the pinch," Proc. Roy. Soc. London, Ser. A., 245, 435 (1958).

${ }^{89}$ Barnes, A., "Collisionless damping of hydromagnetic waves," Phys. Fluids, 9, 1483 (1966).

${ }^{90}$ Fried, B. D., and S. D. Conte, "The Plasma Dispersion Relation," Academic Press, New York (1961).

${ }^{91}$ Gary, S. P., "Theory of Space Plasma Microinstabilities," Cambridge University Press, Cambridge (1993).

${ }^{92}$ McKean., M. E., D. Winske, and S. P. Gary, "Mirror and ion cyclotron anisotropy instabilities in the magnetosheath," J. Geophys. Res., 97, 19421 (1992).

${ }^{93}$ Price, C. P., D. W. Swift, and L.-C. Lee, "Numerical simulations of nonoscillatory mirror waves at the Earth's magnetosheath," J. Geophys. Res., 91, 101 (1986).

${ }^{94}$ Davidson, R. C., and Joan M. Ogden, "Electromagnetic ion cyclotron instability driven by ion energy anisotropy in high-beta plasmas,",Phys. Fluids, 18, 1045 (1975).

${ }^{95}$ Winske, D., and N. Omidi, "Hybrid codes: Methods and applications, in Computer Space Plasma Physics: Simulation Techniques and Software," edited by H. Matsumoto and Y. Omura, Terra Scientific, Tokyo (1992).

${ }^{96}$ McKean, M. E., D. Winske, S. P. Gary, "Two-dimensional simulations of ion anisotropy instabilities in the magnetosheath," J. Geophys. Res., 99, 11,141 (1994).

${ }^{97}$ Gary, S. Peter, Michael E. McKean, Dan Winske, Brian J. Anderson, Richard E. Denton and Stephen A. Fusilier, "The proton cyclotron instability and the anisotropy/ $\beta$ inverse correlation," J. Geophys. Res., 99, 5903 (1994).

${ }^{98}$ Gary, S. Peter. Private Communication (1999).

${ }^{99}$ Gary, S. Peter, Brian J. Anderson, Richard E. Denton, Stephen A. Fuselier and Michael E. McKean, "A limited closure relation for anisotropic plasmas from the Earth's magnetosheath," Phys. Plasmas, 1, 1676 (1994).

${ }^{100}$ Anderson. B. J., and S. A. Fuselier, "Magnetic pulsations from 0.1 to $4.0 \mathrm{~Hz}$ and associated plasma properties in the Earth's subsolar magnetosheath and plasma depletion layer," J. Geophys. Res., 98, 1461 (1993).

${ }^{101}$ Song, Paul, Private Communication (1999).

${ }^{102}$ Anderson, B. J., T. -D. Phan, and S. A. Fuselier, "Relationships between plasma depletion and subsolar reconnection," J. Geophys. Res., 102, 9531 (1997).

103 Anderson, Brian J., Stephan A. Fuselier, S. Peter Gary, and Richard E. Denton, "Magnetic spectral signatures in the Earth's magnetosheath and plasma depletion layer," 99, J. Geophys. Res. 5877 (1994).

${ }^{104}$ Phan, T.-D., G. Paschmann, W. Baumjohann, N. Sckopke, and H. Luhr, "The magnetosheath region adjacent to the dayside magnetopause: AMPTE/IRM observations," J. Geophys. Res., 99, 121 (1994).

${ }^{105}$ Phan, T. D., et al, "The subsolar magnetosheath and magnetopause for high solar wind ram pressure: WIND observations," Geophys. Res. Lett., 23, 1279 (1996). 
${ }^{106}$ Fuselier, Stephan A., Brian J. Anderson, S. Peter Gary, and Richard E. Denton, "Inverse correlations between the ion temperature anisotropy and plasma beta in the Earth's quasi-parallel magnetosheath," J. Geophys. Res., 99, 14,931 (1994).

${ }^{107}$ Inutake, M. et al, " Thermal barrier formation and plasma confinement in the axisymmetrized tandem mirror GAMMA 10," Phys. Rev. Lett., 55, 939 (1985).

${ }^{108}$ Casper, T. A. and Gary R. Smith, "Observation of Alfvén ion-cyclotron fluctuations in the end-cell plasma in the tandem mirror experiment," Physical Review Letters, 48, 1015 (1982).

${ }^{109}$ Smith, G. R., " Alfvén ion-cyclotron instability in tandem-mirror plasmas I," Phys. Fluids, 27, 1499 (1984).

${ }^{110}$ Ichimaru, M., M. Inutake, R. Katsumata, N. Hino, H. Hojo, K. Ishii, T. Tamano and S. Miyoshi, "Relaxation of pressure Anisotropy Due to Alfvén-ion-cycltron fluctuations observed in ion-cyclotronrange-of-frequency-heated mirror plasmas," Physical Review Letters, 70, 2734 (1993).

${ }^{111}$ Katsumata, Ryota, Masaaki Inutake, Makoto Ichimaru, Noriaki Hino, Hideaki Onda, Isao Katanuma, Hitosi Hojo, Atsushi Mase and Syoichi Miyoshi, "Temperature anisotropy measurement using diagmagnetic loop array," Jpn. J. Appl. Phys., 31, 2249 (1992).

${ }^{112}$ Scime, Earl E, Paul A. Keiter, Michael W. Zintl, Matthew M. Balkey, John L. Kline, and Mark E. Koepke, "Control of ion temperature anisotropy in a helicon plasma," Plasma Sources Sci. Technol., 7, 186, (1998).

${ }^{113}$ Huba, J. D., NRL Plasma Formulary (Naval Research Laboratory, Washington D.C. 1994), pp 33.

${ }^{114}$ Phelps, A. V., "Cross sections and swarm coefficients for nitrogen ions and neutrals in $\mathrm{N}_{2}$ and argon ions and neutrals in Ar for energies from $0.1 \mathrm{eV}$ to $10 \mathrm{keV}$," J. Phys. Chem. Ref. Data, 20, 557 (1991). 